Supporting Information

\title{
Lewis Structures from Open Quantum Systems Natural Orbitals: Real Space Adaptive Natural Density Partitioning
}

\author{
Evelio Francisco, * Aurora Costales, María Menéndez-Herrero, \\ and Ángel Martín Pendás*
}

E-mail:evelio@uniovi.es, ampendas@uniovi.es

Departamento de Química Física y Analítica. Facultad de Química. Universidad de

Oviedo. 33006 Oviedo, Spain 


\section{Contents}

S1 Generalized Fragment Natural Orbitals (FNO): Eigenvalues and localization S4

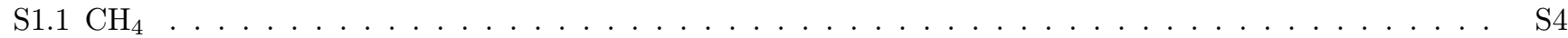

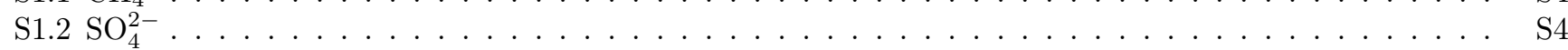

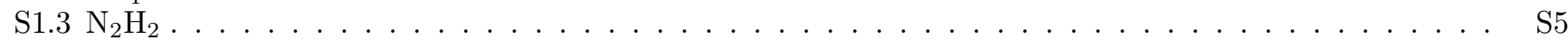

S1.4 The cis-butadiene plus ethylene Diels-Alder $(\mathrm{DA})$ reaction $\ldots \ldots \ldots \ldots \ldots \ldots \ldots \ldots$

S1.4.1 IRC Step $00 \ldots \ldots \ldots \ldots \ldots \ldots \ldots \ldots \ldots \ldots \ldots$

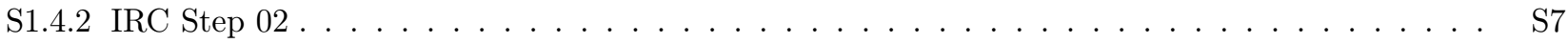

S1.4.3 IRC Step $04 \ldots \ldots \ldots \ldots \ldots \ldots \ldots \ldots \ldots \ldots \ldots \ldots \ldots$

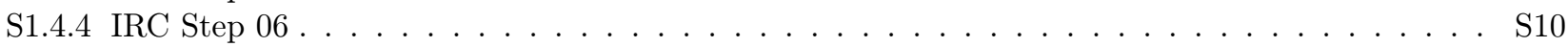

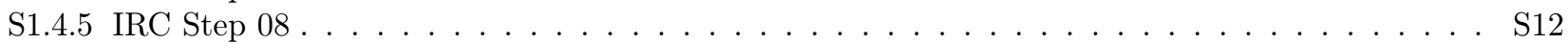

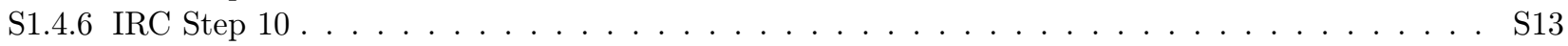

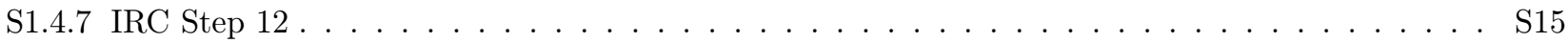

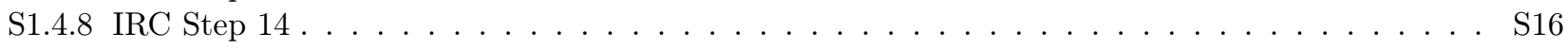

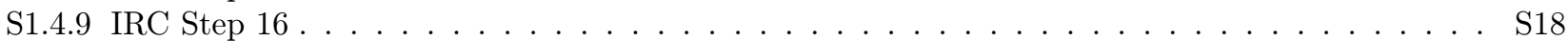

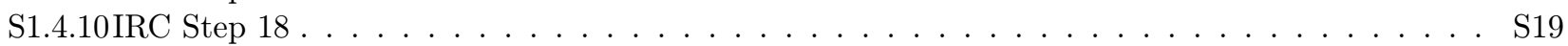

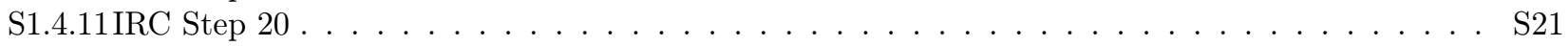

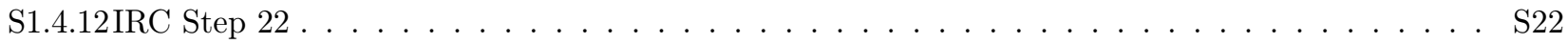

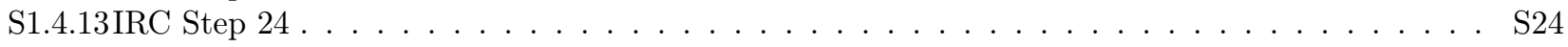

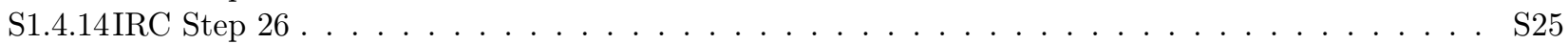

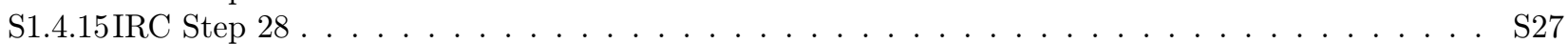

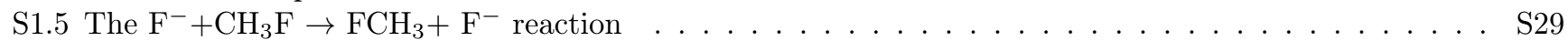

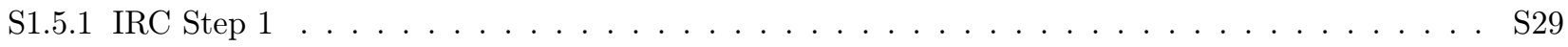

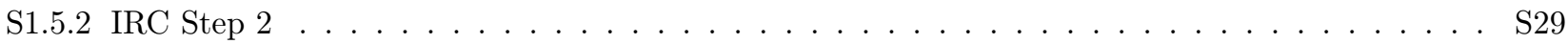

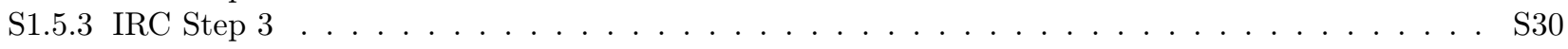

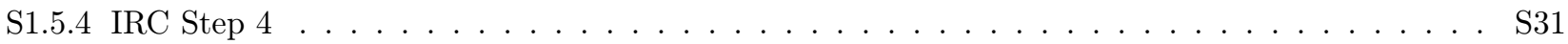

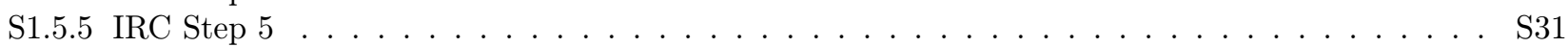

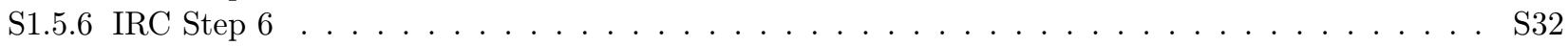

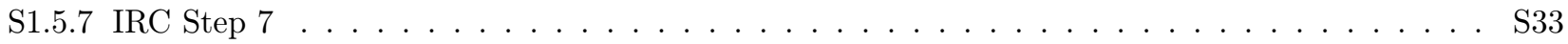

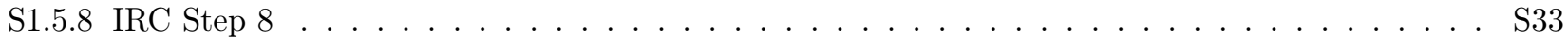

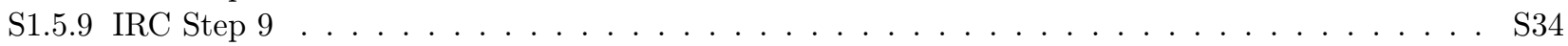

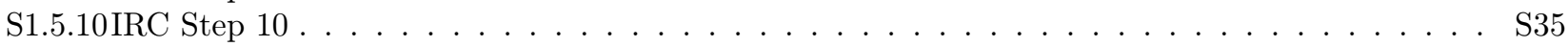

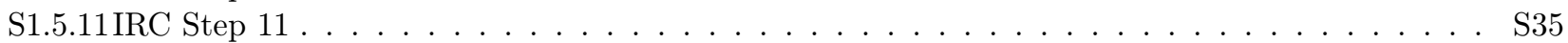

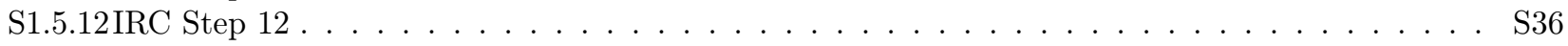

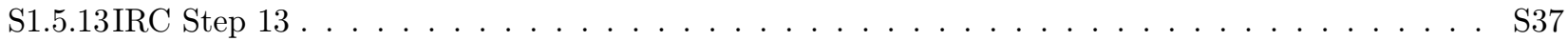

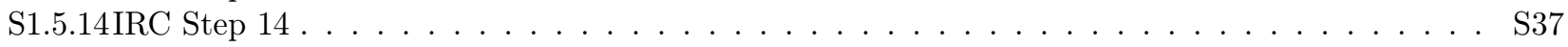

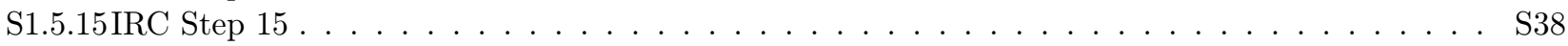

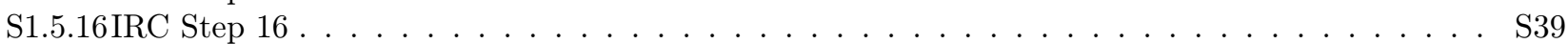

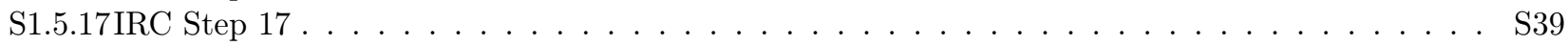

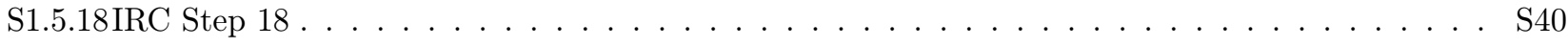

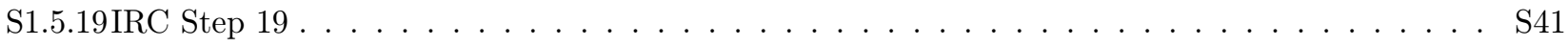

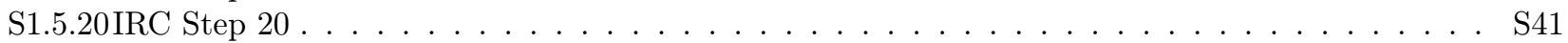

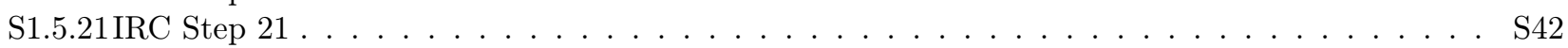

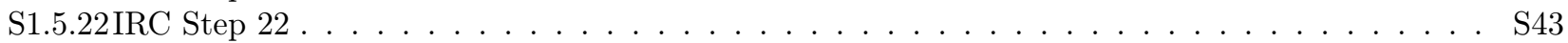

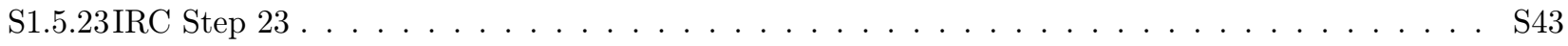

S1.5.24IRC Step $24 \ldots \ldots \ldots \ldots \ldots \ldots \ldots \ldots \ldots \ldots \ldots \ldots \ldots \ldots \ldots$

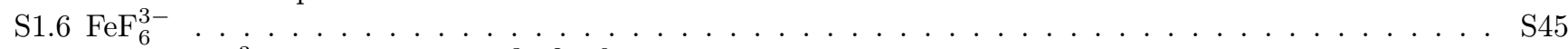

S1.6.1 $\mathrm{FeF}_{6}^{3-}$ Oh High spin, $\left(\mathrm{t}_{2 g}^{3} \mathrm{~g}_{g}^{2}-{ }^{6} A_{1 g}\right) \ldots \ldots \ldots \ldots \ldots \ldots \ldots \ldots \ldots \ldots \ldots \ldots \ldots$

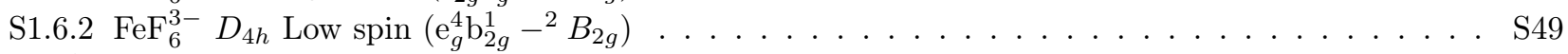

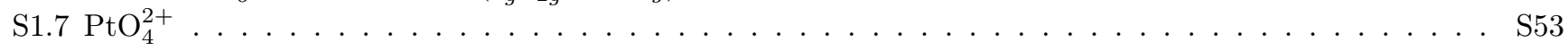

S2 Generalized Fragment Natural Orbitals (FNO): Eigenvectors

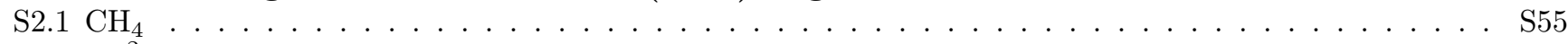

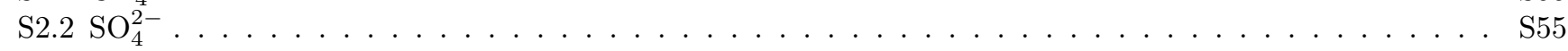

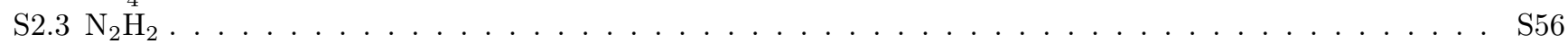

S2.4 The cis-butadiene plus ethylene Diels-Alder $(\mathrm{DA})$ reaction $\ldots \ldots \ldots \ldots \ldots \ldots \ldots \ldots$. . . . . . . . . 557

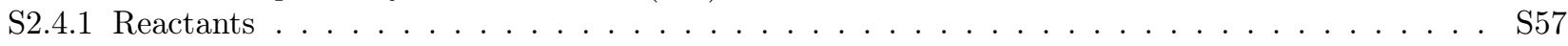

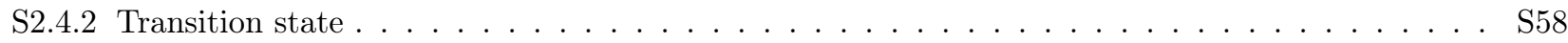

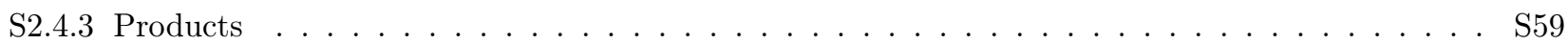

$\mathrm{S} 2.5$ The $\mathrm{F}^{-}+\mathrm{CH}_{3} \mathrm{~F} \rightarrow \mathrm{FCH}_{3}+\mathrm{F}^{-}$reaction $\ldots \ldots \ldots \ldots \ldots \ldots \ldots \ldots \ldots$

S2.5.1 Reactants Products . . . . . . . . . . . . . . . . . . . . . . . S60

S2.5.2 Transition State . . . . . . . . . . . . . . . . . . . . . . . S61 
$\mathrm{S} 2.6 \mathrm{FeF}_{6}^{3-}$ complex in $\mathrm{Oh}$ (high spin) and $D_{4 h}$ (low spin) multielectron states. . . . . . . . . . . . . . S62 $\mathrm{S} 2.6 .1 \mathrm{FeF}_{6}^{3-} \mathrm{Oh}$ High spin, $\left(\mathrm{t}_{2 g}^{3} \mathrm{e}_{g}^{2}-{ }^{6} A_{1 g}\right), \alpha$-orbitals . . . . . . . . . . . . . . . . S62

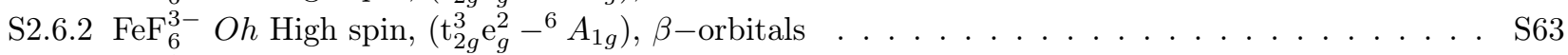

$\mathrm{S} 2.6 .3 \mathrm{FeF}_{6}^{3-} D_{4 h}$ Low spin $\left(\mathrm{e}_{g}^{4} \mathrm{~b}_{2 g}^{1}-{ }^{2} B_{2 g}\right), \alpha$-orbitals . . . . . . . . . . . . . . . . S64

$\mathrm{S} 2.6 .4 \mathrm{FeF}_{6}^{3-} D_{4 h}$ Low spin $\left(\mathrm{e}_{g}^{4} \mathrm{~b}_{2 g}^{1}-{ }^{2} B_{2 g}\right) \beta$-orbitals . . . . . . . . . . . . . . . S65

$\mathrm{S} 2.7 \mathrm{PtO}_{4}^{2+} \ldots \ldots \ldots \ldots \ldots \ldots \ldots \ldots \ldots \ldots$

S3 Cartesian coordinates S67

$\mathrm{S} 3.1 \mathrm{CH}_{4} \ldots \ldots \ldots \ldots \ldots \ldots \ldots \ldots$

$\mathrm{S} 3.2 \mathrm{SO}_{4}^{2-} \ldots \ldots \ldots \ldots \ldots \ldots \ldots$

$\mathrm{S} 3.3 \mathrm{~N}_{2} \mathrm{H}_{2} \ldots \ldots \ldots \ldots \ldots \ldots \ldots$

S3.4 The cis-butadiene plus ethylene Diels-Alder (DA) reaction . . . . . . . . . . . . . . . . . . . S67

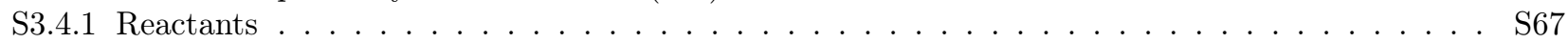

S3.4.2 Transition state . . . . . . . . . . . . . . . . . . . . . . . . S68

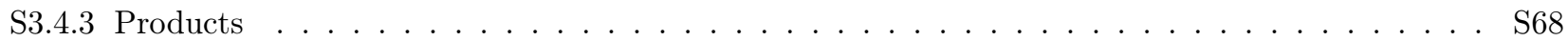

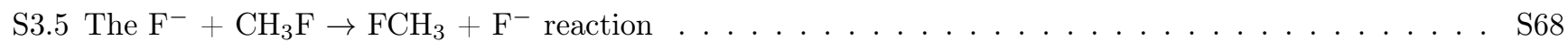

S3.5.1 Reactants/Products . . . . . . . . . . . . . . . . . . . . . S68

S3.5.2 Transition state . . . . . . . . . . . . . . . . . . . . . . . S68

S3.6 $\mathrm{FeF}_{6}^{3-}$ complex in $\mathrm{Oh}$ (high spin) and $D_{4 h}$ (low spin) multielectron states. . . . . . . . . . . . . . . . S69

$\mathrm{S} 3.6 .1 \mathrm{FeF}_{6}^{3-} \mathrm{Oh} \ldots \ldots \ldots \ldots \ldots \ldots \ldots \ldots \ldots \ldots \ldots \ldots$

$\mathrm{S} 3.6 .2 \mathrm{FeF}_{6}^{3-} D_{4 h} \ldots \ldots \ldots \ldots \ldots \ldots \ldots \ldots \ldots$

$\mathrm{S} 3.7 \mathrm{PtO}_{4}^{2+} \ldots \ldots \ldots \ldots \ldots \ldots \ldots \ldots \ldots$ 


\section{S1 Generalized Fragment Natural Orbitals (FNO): Eigenvalues and lo- calization}

$\lambda$ 's, percentage of localization and number of atoms expanded by each FNO for the systems examined. Notice that the numbering of FNOs does not coincide with that in the text.

\section{S1.1 $\mathrm{CH}_{4}$}

\begin{tabular}{ccc}
\hline \multicolumn{3}{c}{ 1-center FNO } \\
\hline MO & $\lambda$ & Atolysis, $\varepsilon=0.95000$ \\
\hline 1 & 0.99998 & $\mathrm{C}_{1}$ \\
\hline 2 -center FNO & analysis, $\varepsilon=0.95000$ \\
\hline MO & $\lambda$ & Atoms \\
\hline 2 & 0.97302 & $\mathrm{C}_{1}-\mathrm{H}_{2}$ \\
3 & 0.97302 & $\mathrm{C}_{1}-\mathrm{H}_{3}$ \\
4 & 0.97302 & $\mathrm{C}_{1}-\mathrm{H}_{4}$ \\
5 & 0.97302 & $\mathrm{C}_{1}-\mathrm{H}_{5}$ \\
\hline \hline
\end{tabular}

\begin{tabular}{crrrrrr}
\hline \multicolumn{6}{c}{ Localization and atoms expanded by each FNO } \\
\hline FNO & Loc $_{1}$ & Loc $_{2}$ & Loc $_{3}$ & Loc $_{4}$ & Loc $_{5}$ & $n_{\text {eff }}$ \\
\hline 1 & $100.0 \%$ & $0.0 \%$ & $0.0 \%$ & $0.0 \%$ & $0.0 \%$ & 1.00004 \\
2 & $49.3 \%$ & $48.0 \%$ & $0.9 \%$ & $0.9 \%$ & $0.9 \%$ & 2.11105 \\
3 & $49.3 \%$ & $0.9 \%$ & $48.0 \%$ & $0.9 \%$ & $0.9 \%$ & 2.11105 \\
4 & $49.3 \%$ & $0.9 \%$ & $0.9 \%$ & $48.0 \%$ & $0.9 \%$ & 2.11105 \\
5 & $49.3 \%$ & $0.9 \%$ & $0.9 \%$ & $0.9 \%$ & $48.0 \%$ & 2.11105 \\
\hline \hline
\end{tabular}

\section{$\mathrm{S} 1.2 \quad \mathrm{SO}_{4}^{2-}$}

\begin{tabular}{ccc}
\hline \multicolumn{3}{c}{ 1-center FNO analysis, $\varepsilon=0.95000$} \\
\hline MO & $\lambda$ & Atoms \\
\hline 1 & 1.00000 & $\mathrm{~S}_{1}$ \\
2 & 0.99844 & $\mathrm{~S}_{1}$ \\
3 & 0.99770 & $\mathrm{~S}_{1}$ \\
4 & 0.99770 & $\mathrm{~S}_{1}$ \\
5 & 0.99770 & $\mathrm{~S}_{1}$ \\
6 & 1.00000 & $\mathrm{O}_{2}$ \\
7 & 0.99758 & $\mathrm{O}_{2}$ \\
8 & 1.00000 & $\mathrm{O}_{3}$ \\
9 & 0.99758 & $\mathrm{O}_{3}$ \\
10 & 1.00000 & $\mathrm{O}_{4}$ \\
11 & 0.99758 & $\mathrm{O}_{4}$ \\
12 & 1.00000 & $\mathrm{O}_{5}$ \\
13 & 0.99758 & $\mathrm{O}_{5}$ \\
\hline \hline 2 -center FNO analysis, $\varepsilon=0.95000$ \\
\hline MO & $\lambda$ & $\mathrm{Atoms}_{1}$ \\
\hline 14 & 0.97088 & $\mathrm{~S}_{1}-\mathrm{O}_{2}$ \\
15 & 0.96985 & $\mathrm{~S}_{1}-\mathrm{O}_{2}$ \\
16 & 0.96985 & $\mathrm{~S}_{1}-\mathrm{O}_{2}$ \\
17 & 0.97088 & $\mathrm{~S}_{1}-\mathrm{O}_{3}$ \\
18 & 0.96985 & $\mathrm{~S}_{1}-\mathrm{O}_{3}$ \\
19 & 0.96985 & $\mathrm{~S}_{1}-\mathrm{O}_{3}$ \\
20 & 0.97088 & $\mathrm{~S}_{1}-\mathrm{O}_{4}$ \\
21 & 0.96985 & $\mathrm{~S}_{1}-\mathrm{O}_{4}$ \\
22 & 0.96985 & $\mathrm{~S}_{1}-\mathrm{O}_{4}$ \\
23 & 0.97088 & $\mathrm{~S}_{1}-\mathrm{O}_{5}$ \\
24 & 0.96985 & $\mathrm{~S}_{1}-\mathrm{O}_{5}$ \\
25 & 0.96985 & $\mathrm{~S}_{1}-\mathrm{O}_{5}$ \\
\hline \hline
\end{tabular}




\begin{tabular}{ccrrrrr}
\hline \multicolumn{7}{c}{ Localization and atoms expanded by each FNO } \\
\hline FNO & \multicolumn{1}{c}{ Loc $_{1}$} & Loc $_{2}$ & Loc $_{3}$ & Loc $_{4}$ & Loc $_{5}$ & $n_{\text {eff }}$ \\
\hline 1 & $100.0 \%$ & $-0.0 \%$ & $-0.0 \%$ & $-0.0 \%$ & $-0.0 \%$ & 1.00000 \\
2 & $99.8 \%$ & $0.0 \%$ & $0.0 \%$ & $0.0 \%$ & $0.0 \%$ & 1.00313 \\
3 & $99.8 \%$ & $0.1 \%$ & $0.1 \%$ & $0.1 \%$ & $0.1 \%$ & 1.00462 \\
4 & $99.8 \%$ & $0.1 \%$ & $0.0 \%$ & $0.0 \%$ & $0.1 \%$ & 1.00462 \\
5 & $99.8 \%$ & $0.0 \%$ & $0.1 \%$ & $0.1 \%$ & $0.0 \%$ & 1.00462 \\
6 & $-0.0 \%$ & $100.0 \%$ & $0.0 \%$ & $0.0 \%$ & $0.0 \%$ & 1.00000 \\
7 & $0.1 \%$ & $99.8 \%$ & $0.0 \%$ & $0.0 \%$ & $0.0 \%$ & 1.00486 \\
8 & $-0.0 \%$ & $0.0 \%$ & $100.0 \%$ & $0.0 \%$ & $0.0 \%$ & 1.00000 \\
9 & $0.1 \%$ & $0.0 \%$ & $99.8 \%$ & $0.0 \%$ & $0.0 \%$ & 1.00486 \\
10 & $-0.0 \%$ & $0.0 \%$ & $0.0 \%$ & $100.0 \%$ & $0.0 \%$ & 1.00000 \\
11 & $0.1 \%$ & $0.0 \%$ & $0.0 \%$ & $99.8 \%$ & $0.0 \%$ & 1.00486 \\
12 & $-0.0 \%$ & $0.0 \%$ & $0.0 \%$ & $0.0 \%$ & $100.0 \%$ & 1.00000 \\
13 & $0.1 \%$ & $0.0 \%$ & $0.0 \%$ & $0.0 \%$ & $99.8 \%$ & 1.00486 \\
14 & $18.9 \%$ & $78.1 \%$ & $1.0 \%$ & $1.0 \%$ & $1.0 \%$ & 1.54617 \\
15 & $4.5 \%$ & $92.4 \%$ & $0.6 \%$ & $1.3 \%$ & $1.3 \%$ & 1.16748 \\
16 & $4.5 \%$ & $92.4 \%$ & $1.5 \%$ & $0.8 \%$ & $0.8 \%$ & 1.16748 \\
17 & $18.9 \%$ & $1.0 \%$ & $78.1 \%$ & $1.0 \%$ & $1.0 \%$ & 1.54617 \\
18 & $4.5 \%$ & $0.6 \%$ & $92.4 \%$ & $1.3 \%$ & $1.3 \%$ & 1.16748 \\
19 & $4.5 \%$ & $1.5 \%$ & $92.4 \%$ & $0.8 \%$ & $0.8 \%$ & 1.16748 \\
20 & $18.9 \%$ & $1.0 \%$ & $1.0 \%$ & $78.1 \%$ & $1.0 \%$ & 1.54617 \\
21 & $4.5 \%$ & $1.3 \%$ & $1.3 \%$ & $92.4 \%$ & $0.6 \%$ & 1.16748 \\
22 & $4.5 \%$ & $0.8 \%$ & $0.8 \%$ & $92.4 \%$ & $1.5 \%$ & 1.16748 \\
23 & $18.9 \%$ & $1.0 \%$ & $1.0 \%$ & $1.0 \%$ & $78.1 \%$ & 1.54617 \\
24 & $4.5 \%$ & $1.3 \%$ & $1.3 \%$ & $0.6 \%$ & $92.4 \%$ & 1.16748 \\
25 & $4.5 \%$ & $0.8 \%$ & $0.8 \%$ & $1.5 \%$ & $92.4 \%$ & 1.16748 \\
\hline \hline
\end{tabular}

\section{$\mathbf{S 1 . 3} \quad \mathbf{N}_{2} \mathbf{H}_{2}$}

\begin{tabular}{|c|c|c|c|c|c|}
\hline \multicolumn{6}{|c|}{ 1-center FNO analysis, $\varepsilon=0.85000$} \\
\hline $\mathrm{MO}$ & $\lambda$ & \multicolumn{2}{|l|}{ Atoms } & & \\
\hline 1 & 1.00000 & \multicolumn{2}{|l|}{$\mathrm{N}_{1}$} & & \\
\hline 2 & 0.98541 & \multicolumn{2}{|l|}{$\mathrm{N}_{1}$} & & \\
\hline 3 & 1.00000 & \multicolumn{2}{|l|}{$\mathrm{N}_{2}$} & & \\
\hline 4 & 0.98541 & \multicolumn{2}{|l|}{$\mathrm{N}_{2}$} & & \\
\hline \multicolumn{6}{|c|}{ 2-center FNO analysis, $\varepsilon=0.85000$} \\
\hline $\mathrm{MO}$ & $\lambda$ & \multicolumn{2}{|l|}{ Atoms } & & \\
\hline 5 & 0.95550 & \multicolumn{2}{|l|}{$\mathrm{N}_{1}-\mathrm{N}_{2}$} & & \\
\hline 6 & 0.87797 & \multicolumn{2}{|l|}{$\mathrm{N}_{1}-\mathrm{N}_{2}$} & & \\
\hline 7 & 0.98805 & \multicolumn{2}{|l|}{$\mathrm{N}_{1}-\mathrm{H}_{3}$} & & \\
\hline 8 & 0.98805 & \multicolumn{2}{|l|}{$\mathrm{N}_{2}-\mathrm{H}_{4}$} & & \\
\hline \multicolumn{6}{|c|}{ L Localization and atoms expanded by each FNO } \\
\hline FNO & $\overline{\mathrm{Loc}_{1}}$ & $\overline{\mathrm{LOC}_{2}}$ & $\mathrm{Loc}_{3}$ & $\mathrm{Loc}_{4}$ & $n_{\text {eff }}$ \\
\hline 1 & $100.0 \%$ & $0.0 \%$ & $0.0 \%$ & $0.0 \%$ & 1.00000 \\
\hline 2 & $98.8 \%$ & $0.8 \%$ & $0.3 \%$ & $0.1 \%$ & 1.02348 \\
\hline 3 & $0.0 \%$ & $100.0 \%$ & $0.0 \%$ & $0.0 \%$ & 1.00000 \\
\hline 4 & $0.8 \%$ & $98.8 \%$ & $0.1 \%$ & $0.3 \%$ & 1.02348 \\
\hline 5 & $49.5 \%$ & $49.5 \%$ & $0.5 \%$ & $0.5 \%$ & 2.04294 \\
\hline 6 & $49.3 \%$ & $49.3 \%$ & $0.7 \%$ & $0.7 \%$ & 2.04294 \\
\hline 7 & $69.4 \%$ & $1.1 \%$ & $29.4 \%$ & $0.1 \%$ & 1.76675 \\
\hline 8 & $1.1 \%$ & $69.4 \%$ & $0.1 \%$ & $29.4 \%$ & 1.76675 \\
\hline
\end{tabular}




\section{S1.4 The cis-butadiene plus ethylene Diels-Alder (DA) reaction}

The IRC has been projected onto the $\mathrm{C}-\mathrm{C}$ distance $(\mathrm{R})$ of any of the two single $\sigma$ bonds that are formed during the cycloaddition. In the next subsections the evolution with $R$ of the eigenvalues and effective number of centers for all FNOs is show.

\section{S1.4.1 IRC Step 00}

\begin{tabular}{ccl}
\hline \hline \multicolumn{3}{c}{ 1-center FNO analysis, $\varepsilon=0.90000$} \\
\hline MO & \multicolumn{1}{c}{$\lambda$} & Atoms \\
\hline 1 & 0.99999 & $\mathrm{C}_{1}$ \\
2 & 0.99999 & $\mathrm{C}_{2}$ \\
3 & 0.99999 & $\mathrm{C}_{5}$ \\
4 & 0.99999 & $\mathrm{C}_{6}$ \\
5 & 0.99999 & $\mathrm{C}_{11}$ \\
6 & 0.99999 & $\mathrm{C}_{12}$ \\
\hline \hline 2 -center FNO analysis, $=0.90000$ \\
\hline MO & \multicolumn{3}{c}{$\lambda$} & Atoms $^{2}$ \\
\hline 7 & 0.97468 & $\mathrm{C}_{1}-\mathrm{C}_{2}$ \\
8 & 0.97485 & $\mathrm{C}_{1}-\mathrm{H}_{3}$ \\
9 & 0.98210 & $\mathrm{C}_{1}-\mathrm{C}_{5}$ \\
10 & 0.97473 & $\mathrm{C}_{2}-\mathrm{H}_{4}$ \\
11 & 0.98206 & $\mathrm{C}_{2}-\mathrm{C}_{6}$ \\
12 & 0.97550 & $\mathrm{C}_{5}-\mathrm{H}_{7}$ \\
13 & 0.98067 & $\mathrm{C}_{5}-\mathrm{H}_{9}$ \\
14 & 0.97549 & $\mathrm{C}_{6}-\mathrm{H}_{8}$ \\
15 & 0.98067 & $\mathrm{C}_{6}-\mathrm{H}_{10}$ \\
16 & 0.98360 & $\mathrm{C}_{11}-\mathrm{C}_{12}$ \\
17 & 0.92050 & $\mathrm{C}_{11}-\mathrm{C}_{12}$ \\
18 & 0.98030 & $\mathrm{C}_{11}-\mathrm{H}_{13}$ \\
19 & 0.97902 & $\mathrm{C}_{11}-\mathrm{H}_{15}$ \\
20 & 0.98026 & $\mathrm{C}_{12}-\mathrm{H}_{14}$ \\
21 & 0.97903 & $\mathrm{C}_{12}-\mathrm{H}_{16}$ \\
\hline \hline 3 -center FNO analysis, $\varepsilon=0.90000^{\mathrm{MO}}$ & $\lambda$ & Atoms $_{1}$ \\
\hline 22 & 0.94317 & $\mathrm{C}_{1}-\mathrm{C}_{2}-\mathrm{C}_{5}$ \\
23 & 0.94317 & $\mathrm{C}_{1}-\mathrm{C}_{2}-\mathrm{C}_{6}$ \\
\hline \hline
\end{tabular}

\begin{tabular}{|c|c|c|c|c|c|c|c|c|c|}
\hline \multicolumn{10}{|c|}{ "Localization and atoms expanded by each FNO } \\
\hline FNO & $\mathrm{Loc}_{1}$ & $\mathrm{Loc}_{2}$ & $\mathrm{LOc}_{3}$ & $\mathrm{Loc}_{4}$ & $\mathrm{LOC}_{5}$ & $\mathrm{Loc}_{6}$ & $\mathrm{Loc}_{7}$ & $\mathrm{Loc}_{8}$ & $\mathrm{Loc}_{9}$ \\
\hline 1 & $100.0 \%$ & $0.0 \%$ & $0.0 \%$ & $0.0 \%$ & $0.0 \%$ & $0.0 \%$ & $0.0 \%$ & $0.0 \%$ & $0.0 \%$ \\
\hline 2 & $0.0 \%$ & $100.0 \%$ & $0.0 \%$ & $0.0 \%$ & $0.0 \%$ & $0.0 \%$ & $0.0 \%$ & $0.0 \%$ & $0.0 \%$ \\
\hline 3 & $0.0 \%$ & $0.0 \%$ & $0.0 \%$ & $0.0 \%$ & $100.0 \%$ & $0.0 \%$ & $0.0 \%$ & $0.0 \%$ & $0.0 \%$ \\
\hline 4 & $0.0 \%$ & $0.0 \%$ & $0.0 \%$ & $0.0 \%$ & $0.0 \%$ & $100.0 \%$ & $0.0 \%$ & $0.0 \%$ & $0.0 \%$ \\
\hline 5 & $-0.0 \%$ & $0.0 \%$ & $0.0 \%$ & $0.0 \%$ & $0.0 \%$ & $0.0 \%$ & $0.0 \%$ & $0.0 \%$ & $0.0 \%$ \\
\hline 6 & $-0.0 \%$ & $0.0 \%$ & $0.0 \%$ & $0.0 \%$ & $0.0 \%$ & $0.0 \%$ & $0.0 \%$ & $0.0 \%$ & $0.0 \%$ \\
\hline 7 & $48.7 \%$ & $48.7 \%$ & $0.5 \%$ & $0.5 \%$ & $0.6 \%$ & $0.6 \%$ & $0.1 \%$ & $0.1 \%$ & $0.1 \%$ \\
\hline 8 & $51.8 \%$ & $0.9 \%$ & $45.7 \%$ & $0.2 \%$ & $1.0 \%$ & $0.1 \%$ & $0.1 \%$ & $0.0 \%$ & $0.2 \%$ \\
\hline 9 & $49.2 \%$ & $0.4 \%$ & $0.4 \%$ & $0.1 \%$ & $49.0 \%$ & $0.1 \%$ & $0.4 \%$ & $0.0 \%$ & $0.4 \%$ \\
\hline 10 & $0.9 \%$ & $51.8 \%$ & $0.2 \%$ & $45.7 \%$ & $0.1 \%$ & $1.0 \%$ & $0.0 \%$ & $0.1 \%$ & $0.0 \%$ \\
\hline 11 & $0.4 \%$ & $49.2 \%$ & $0.1 \%$ & $0.4 \%$ & $0.1 \%$ & $49.0 \%$ & $0.0 \%$ & $0.4 \%$ & $0.0 \%$ \\
\hline 12 & $0.8 \%$ & $0.1 \%$ & $0.1 \%$ & $0.0 \%$ & $53.0 \%$ & $0.1 \%$ & $44.5 \%$ & $0.3 \%$ & $0.6 \%$ \\
\hline 13 & $0.9 \%$ & $0.1 \%$ & $0.2 \%$ & $0.0 \%$ & $53.1 \%$ & $0.0 \%$ & $0.6 \%$ & $0.0 \%$ & $44.9 \%$ \\
\hline 14 & $0.1 \%$ & $0.8 \%$ & $0.0 \%$ & $0.1 \%$ & $0.1 \%$ & $53.0 \%$ & $0.3 \%$ & $44.5 \%$ & $0.0 \%$ \\
\hline 15 & $0.1 \%$ & $0.9 \%$ & $0.0 \%$ & $0.2 \%$ & $0.0 \%$ & $53.1 \%$ & $0.0 \%$ & $0.6 \%$ & $0.0 \%$ \\
\hline 16 & $0.0 \%$ & $0.0 \%$ & $0.0 \%$ & $0.0 \%$ & $0.0 \%$ & $0.0 \%$ & $0.0 \%$ & $0.0 \%$ & $0.0 \%$ \\
\hline 17 & $0.2 \%$ & $0.2 \%$ & $0.0 \%$ & $0.0 \%$ & $0.4 \%$ & $0.4 \%$ & $0.2 \%$ & $0.2 \%$ & $0.0 \%$ \\
\hline 18 & $0.0 \%$ & $0.0 \%$ & $0.0 \%$ & $0.0 \%$ & $0.1 \%$ & $0.0 \%$ & $0.1 \%$ & $0.0 \%$ & $0.0 \%$ \\
\hline 19 & $0.1 \%$ & $0.0 \%$ & $0.0 \%$ & $0.0 \%$ & $0.2 \%$ & $0.0 \%$ & $0.0 \%$ & $0.0 \%$ & $0.0 \%$ \\
\hline 20 & $0.0 \%$ & $0.0 \%$ & $0.0 \%$ & $0.0 \%$ & $0.0 \%$ & $0.1 \%$ & $0.0 \%$ & $0.1 \%$ & $0.0 \%$ \\
\hline 21 & $0.0 \%$ & $0.1 \%$ & $0.0 \%$ & $0.0 \%$ & $0.0 \%$ & $0.2 \%$ & $0.0 \%$ & $0.0 \%$ & $0.0 \%$ \\
\hline 22 & $46.1 \%$ & $7.6 \%$ & $1.6 \%$ & $0.2 \%$ & $40.6 \%$ & $0.3 \%$ & $1.2 \%$ & $0.0 \%$ & $1.4 \%$ \\
\hline 23 & $7.7 \%$ & $46.1 \%$ & $0.2 \%$ & $1.6 \%$ & $0.3 \%$ & $40.6 \%$ & $0.0 \%$ & $1.2 \%$ & $0.0 \%$ \\
\hline
\end{tabular}




\begin{tabular}{crrrrrrrr}
\hline \multicolumn{2}{l}{ localization and atoms expanded by each FNO } \\
\hline FNO & Loc $_{10}$ & Loc $_{11}$ & Loc $_{12}$ & Loc $_{13}$ & Loc $_{14}$ & Loc $_{15}$ & Loc $_{16}$ & $n_{\text {eff }}$ \\
\hline 1 & $0.0 \%$ & $0.0 \%$ & $0.0 \%$ & $0.0 \%$ & $0.0 \%$ & $0.0 \%$ & $0.0 \%$ & 1.00002 \\
2 & $0.0 \%$ & $0.0 \%$ & $0.0 \%$ & $0.0 \%$ & $0.0 \%$ & $0.0 \%$ & $0.0 \%$ & 1.00002 \\
3 & $0.0 \%$ & $0.0 \%$ & $0.0 \%$ & $0.0 \%$ & $0.0 \%$ & $0.0 \%$ & $0.0 \%$ & 1.00002 \\
4 & $0.0 \%$ & $0.0 \%$ & $0.0 \%$ & $0.0 \%$ & $0.0 \%$ & $0.0 \%$ & $0.0 \%$ & 1.00002 \\
5 & $0.0 \%$ & $100.0 \%$ & $0.0 \%$ & $0.0 \%$ & $0.0 \%$ & $0.0 \%$ & $0.0 \%$ & 1.00002 \\
6 & $0.0 \%$ & $0.0 \%$ & $100.0 \%$ & $0.0 \%$ & $0.0 \%$ & $0.0 \%$ & $0.0 \%$ & 1.00002 \\
7 & $0.1 \%$ & $0.0 \%$ & $0.0 \%$ & $0.0 \%$ & $0.0 \%$ & $0.0 \%$ & $0.0 \%$ & 2.10472 \\
8 & $0.0 \%$ & $0.0 \%$ & $0.0 \%$ & $0.0 \%$ & $0.0 \%$ & $0.0 \%$ & $0.0 \%$ & 2.09531 \\
9 & $0.0 \%$ & $0.0 \%$ & $0.0 \%$ & $0.0 \%$ & $0.0 \%$ & $0.0 \%$ & $0.0 \%$ & 2.07328 \\
10 & $0.2 \%$ & $0.0 \%$ & $0.0 \%$ & $0.0 \%$ & $0.0 \%$ & $0.0 \%$ & $0.0 \%$ & 2.09585 \\
11 & $0.4 \%$ & $0.0 \%$ & $0.0 \%$ & $0.0 \%$ & $0.0 \%$ & $0.0 \%$ & $0.0 \%$ & 2.07342 \\
12 & $0.0 \%$ & $0.2 \%$ & $0.0 \%$ & $0.1 \%$ & $0.0 \%$ & $0.0 \%$ & $0.0 \%$ & 2.08528 \\
13 & $0.0 \%$ & $0.0 \%$ & $0.0 \%$ & $0.0 \%$ & $0.0 \%$ & $0.0 \%$ & $0.0 \%$ & 2.06456 \\
14 & $0.6 \%$ & $0.0 \%$ & $0.2 \%$ & $0.0 \%$ & $0.1 \%$ & $0.0 \%$ & $0.0 \%$ & 2.08531 \\
15 & $44.9 \%$ & $0.0 \%$ & $0.0 \%$ & $0.0 \%$ & $0.0 \%$ & $0.0 \%$ & $0.0 \%$ & 2.06460 \\
16 & $0.0 \%$ & $49.2 \%$ & $49.2 \%$ & $0.4 \%$ & $0.4 \%$ & $0.4 \%$ & $0.4 \%$ & 2.06698 \\
17 & $0.0 \%$ & $46.0 \%$ & $46.0 \%$ & $1.6 \%$ & $1.6 \%$ & $1.5 \%$ & $1.5 \%$ & 2.35483 \\
18 & $0.0 \%$ & $52.7 \%$ & $0.9 \%$ & $45.3 \%$ & $0.1 \%$ & $0.6 \%$ & $0.1 \%$ & 2.06879 \\
19 & $0.0 \%$ & $53.1 \%$ & $0.9 \%$ & $0.6 \%$ & $0.1 \%$ & $44.8 \%$ & $0.1 \%$ & 2.07138 \\
20 & $0.0 \%$ & $0.9 \%$ & $52.7 \%$ & $0.1 \%$ & $45.3 \%$ & $0.1 \%$ & $0.6 \%$ & 2.06898 \\
21 & $0.0 \%$ & $0.9 \%$ & $53.1 \%$ & $0.1 \%$ & $0.6 \%$ & $0.1 \%$ & $44.8 \%$ & 2.07138 \\
22 & $0.0 \%$ & $0.5 \%$ & $0.1 \%$ & $0.0 \%$ & $0.0 \%$ & $0.3 \%$ & $0.0 \%$ & 2.60685 \\
23 & $1.4 \%$ & $0.1 \%$ & $0.5 \%$ & $0.0 \%$ & $0.0 \%$ & $0.0 \%$ & $0.3 \%$ & 2.60772 \\
\hline \hline
\end{tabular}

\section{S1.4.2 IRC Step 02}

\begin{tabular}{|c|c|c|}
\hline \multicolumn{3}{|c|}{1 1-center FNO analysis, $\varepsilon=0.90000$} \\
\hline $\mathrm{MO}$ & $\lambda$ & Atoms \\
\hline 1 & 0.99999 & $\mathrm{C}_{1}$ \\
\hline 2 & 0.99999 & $\mathrm{C}_{2}$ \\
\hline 3 & 0.99999 & $\mathrm{C}_{5}$ \\
\hline 4 & 0.99999 & $\mathrm{C}_{6}$ \\
\hline 5 & 0.99999 & $\mathrm{C}_{11}$ \\
\hline 6 & 0.99999 & $\mathrm{C}_{12}$ \\
\hline \multicolumn{3}{|c|}{ 2-center FNO analysis, $\varepsilon=0.90000$} \\
\hline MO & $\lambda$ & Atoms \\
\hline 7 & 0.97462 & $\mathrm{C}_{1}-\mathrm{C}_{2}$ \\
\hline 8 & 0.97483 & $\mathrm{C}_{1}-\mathrm{H}_{3}$ \\
\hline 9 & 0.98201 & $\mathrm{C}_{1}-\mathrm{C}_{5}$ \\
\hline 10 & 0.97469 & $\mathrm{C}_{2}-\mathrm{H}_{4}$ \\
\hline 11 & 0.98196 & $\mathrm{C}_{2}-\mathrm{C}_{6}$ \\
\hline 12 & 0.97511 & $\mathrm{C}_{5}-\mathrm{H}_{7}$ \\
\hline 13 & 0.98063 & $\mathrm{C}_{5}-\mathrm{H}_{9}$ \\
\hline 14 & 0.97508 & $\mathrm{C}_{6}-\mathrm{H}_{8}$ \\
\hline 15 & 0.98064 & $\mathrm{C}_{6}-\mathrm{H}_{10}$ \\
\hline 16 & 0.98347 & $\mathrm{C}_{11}-\mathrm{C}_{12}$ \\
\hline 17 & 0.91860 & $\mathrm{C}_{11}-\mathrm{C}_{12}$ \\
\hline 18 & 0.97975 & $\mathrm{C}_{11}-\mathrm{H}_{13}$ \\
\hline 19 & 0.97869 & $\mathrm{C}_{11}-\mathrm{H}_{15}$ \\
\hline 20 & 0.97976 & $\mathrm{C}_{12}-\mathrm{H}_{14}$ \\
\hline 21 & 0.97871 & $\mathrm{C}_{12}-\mathrm{H}_{16}$ \\
\hline \multicolumn{3}{|c|}{3 -center FNO analysis, $\varepsilon=0.90000$} \\
\hline $\mathrm{MO}$ & $\lambda$ & Atoms \\
\hline 22 & 0.94193 & $\mathrm{C}_{1}-\mathrm{C}_{2}-\mathrm{C}_{5}$ \\
\hline 23 & 0.94192 & $\mathrm{C}_{1}-\mathrm{C}_{2}-\mathrm{C}_{6}$ \\
\hline
\end{tabular}




\begin{tabular}{|c|c|c|c|c|c|c|c|c|c|}
\hline \multicolumn{10}{|c|}{ Localization and atoms expanded by each FNO } \\
\hline FNO & $\mathrm{Loc}_{1}$ & $\mathrm{Loc}_{2}$ & $\mathrm{LOc}_{3}$ & $\mathrm{Loc}_{4}$ & $\mathrm{Loc}_{5}$ & $\mathrm{Loc}_{6}$ & $\mathrm{LOC}_{7}$ & $\mathrm{Loc}_{8}$ & $\mathrm{Loc}_{9}$ \\
\hline 1 & $100.0 \%$ & $0.0 \%$ & $0.0 \%$ & $0.0 \%$ & $0.0 \%$ & $0.0 \%$ & $0.0 \%$ & $0.0 \%$ & $0.0 \%$ \\
\hline 2 & $0.0 \%$ & $100.0 \%$ & $0.0 \%$ & $0.0 \%$ & $0.0 \%$ & $0.0 \%$ & $0.0 \%$ & $0.0 \%$ & $0.0 \%$ \\
\hline 3 & $0.0 \%$ & $0.0 \%$ & $0.0 \%$ & $0.0 \%$ & $100.0 \%$ & $0.0 \%$ & $0.0 \%$ & $0.0 \%$ & $0.0 \%$ \\
\hline 4 & $0.0 \%$ & $0.0 \%$ & $0.0 \%$ & $0.0 \%$ & $0.0 \%$ & $100.0 \%$ & $0.0 \%$ & $0.0 \%$ & $0.0 \%$ \\
\hline 5 & $-0.0 \%$ & $0.0 \%$ & $0.0 \%$ & $0.0 \%$ & $0.0 \%$ & $0.0 \%$ & $0.0 \%$ & $0.0 \%$ & $0.0 \%$ \\
\hline 6 & $-0.0 \%$ & $0.0 \%$ & $0.0 \%$ & $0.0 \%$ & $0.0 \%$ & $0.0 \%$ & $0.0 \%$ & $0.0 \%$ & $0.0 \%$ \\
\hline 7 & $48.7 \%$ & $48.7 \%$ & $0.5 \%$ & $0.5 \%$ & $0.6 \%$ & $0.6 \%$ & $0.1 \%$ & $0.1 \%$ & $0.1 \%$ \\
\hline 8 & $51.8 \%$ & $0.9 \%$ & $45.7 \%$ & $0.2 \%$ & $1.0 \%$ & $0.1 \%$ & $0.1 \%$ & $0.0 \%$ & $0.2 \%$ \\
\hline 9 & $49.2 \%$ & $0.4 \%$ & $0.4 \%$ & $0.1 \%$ & $49.0 \%$ & $0.1 \%$ & $0.4 \%$ & $0.0 \%$ & $0.4 \%$ \\
\hline 10 & $0.9 \%$ & $51.8 \%$ & $0.2 \%$ & $45.7 \%$ & $0.1 \%$ & $1.0 \%$ & $0.0 \%$ & $0.1 \%$ & $0.0 \%$ \\
\hline 11 & $0.4 \%$ & $49.2 \%$ & $0.1 \%$ & $0.4 \%$ & $0.1 \%$ & $49.0 \%$ & $0.0 \%$ & $0.4 \%$ & $0.0 \%$ \\
\hline 12 & $0.8 \%$ & $0.1 \%$ & $0.1 \%$ & $0.0 \%$ & $53.0 \%$ & $0.1 \%$ & $44.5 \%$ & $0.3 \%$ & $0.6 \%$ \\
\hline 13 & $0.9 \%$ & $0.1 \%$ & $0.2 \%$ & $0.0 \%$ & $53.1 \%$ & $0.0 \%$ & $0.6 \%$ & $0.0 \%$ & $44.9 \%$ \\
\hline 14 & $0.1 \%$ & $0.8 \%$ & $0.0 \%$ & $0.1 \%$ & $0.1 \%$ & $53.0 \%$ & $0.3 \%$ & $44.5 \%$ & $0.0 \%$ \\
\hline 15 & $0.1 \%$ & $0.9 \%$ & $0.0 \%$ & $0.2 \%$ & $0.0 \%$ & $53.1 \%$ & $0.0 \%$ & $0.6 \%$ & $0.0 \%$ \\
\hline 16 & $0.0 \%$ & $0.0 \%$ & $0.0 \%$ & $0.0 \%$ & $0.0 \%$ & $0.0 \%$ & $0.0 \%$ & $0.0 \%$ & $0.0 \%$ \\
\hline 17 & $0.3 \%$ & $0.3 \%$ & $0.0 \%$ & $0.0 \%$ & $0.5 \%$ & $0.5 \%$ & $0.2 \%$ & $0.2 \%$ & $0.0 \%$ \\
\hline 18 & $0.0 \%$ & $0.0 \%$ & $0.0 \%$ & $0.0 \%$ & $0.1 \%$ & $0.0 \%$ & $0.1 \%$ & $0.0 \%$ & $0.0 \%$ \\
\hline 19 & $0.1 \%$ & $0.0 \%$ & $0.0 \%$ & $0.0 \%$ & $0.2 \%$ & $0.0 \%$ & $0.0 \%$ & $0.0 \%$ & $0.0 \%$ \\
\hline 20 & $0.0 \%$ & $0.0 \%$ & $0.0 \%$ & $0.0 \%$ & $0.0 \%$ & $0.1 \%$ & $0.0 \%$ & $0.1 \%$ & $0.0 \%$ \\
\hline 21 & $0.0 \%$ & $0.1 \%$ & $0.0 \%$ & $0.0 \%$ & $0.0 \%$ & $0.2 \%$ & $0.0 \%$ & $0.0 \%$ & $0.0 \%$ \\
\hline 22 & $46.0 \%$ & $7.8 \%$ & $1.6 \%$ & $0.2 \%$ & $40.4 \%$ & $0.3 \%$ & $1.2 \%$ & $0.0 \%$ & $1.4 \%$ \\
\hline 23 & $7.8 \%$ & $46.0 \%$ & $0.2 \%$ & $1.6 \%$ & $0.3 \%$ & $40.4 \%$ & $0.0 \%$ & $1.2 \%$ & $0.0 \%$ \\
\hline
\end{tabular}

\begin{tabular}{|c|c|c|c|c|c|c|c|c|}
\hline \multicolumn{9}{|c|}{ Localization and atoms expanded by each FNO } \\
\hline FNO & $\operatorname{Loc}_{10}$ & $\operatorname{Loc}_{11}$ & $\operatorname{Loc}_{12}$ & $\operatorname{Loc}_{13}$ & $\mathrm{Loc}_{14}$ & $\operatorname{Loc}_{15}$ & $\operatorname{Loc}_{16}$ & $n_{\text {eff }}$ \\
\hline 1 & $0.0 \%$ & $0.0 \%$ & $0.0 \%$ & $0.0 \%$ & $0.0 \%$ & $0.0 \%$ & $0.0 \%$ & 1.00002 \\
\hline 2 & $0.0 \%$ & $0.0 \%$ & $0.0 \%$ & $0.0 \%$ & $0.0 \%$ & $0.0 \%$ & $0.0 \%$ & 1.00002 \\
\hline 3 & $0.0 \%$ & $0.0 \%$ & $0.0 \%$ & $0.0 \%$ & $0.0 \%$ & $0.0 \%$ & $0.0 \%$ & 1.00002 \\
\hline 4 & $0.0 \%$ & $0.0 \%$ & $0.0 \%$ & $0.0 \%$ & $0.0 \%$ & $0.0 \%$ & $0.0 \%$ & 1.00002 \\
\hline 5 & $0.0 \%$ & $100.0 \%$ & $0.0 \%$ & $0.0 \%$ & $0.0 \%$ & $0.0 \%$ & $0.0 \%$ & 1.00002 \\
\hline 6 & $0.0 \%$ & $0.0 \%$ & $100.0 \%$ & $0.0 \%$ & $0.0 \%$ & $0.0 \%$ & $0.0 \%$ & 1.00002 \\
\hline 7 & $0.1 \%$ & $0.0 \%$ & $0.0 \%$ & $0.0 \%$ & $0.0 \%$ & $0.0 \%$ & $0.0 \%$ & 2.10497 \\
\hline 8 & $0.0 \%$ & $0.0 \%$ & $0.0 \%$ & $0.0 \%$ & $0.0 \%$ & $0.0 \%$ & $0.0 \%$ & 2.09543 \\
\hline 9 & $0.0 \%$ & $0.0 \%$ & $0.0 \%$ & $0.0 \%$ & $0.0 \%$ & $0.0 \%$ & $0.0 \%$ & 2.07364 \\
\hline 10 & $0.2 \%$ & $0.0 \%$ & $0.0 \%$ & $0.0 \%$ & $0.0 \%$ & $0.0 \%$ & $0.0 \%$ & 2.09606 \\
\hline 11 & $0.4 \%$ & $0.0 \%$ & $0.0 \%$ & $0.0 \%$ & $0.0 \%$ & $0.0 \%$ & $0.0 \%$ & 2.07386 \\
\hline 12 & $0.0 \%$ & $0.2 \%$ & $0.0 \%$ & $0.1 \%$ & $0.0 \%$ & $0.0 \%$ & $0.0 \%$ & 2.08693 \\
\hline 13 & $0.0 \%$ & $0.0 \%$ & $0.0 \%$ & $0.0 \%$ & $0.0 \%$ & $0.0 \%$ & $0.0 \%$ & 2.06477 \\
\hline 14 & $0.6 \%$ & $0.0 \%$ & $0.2 \%$ & $0.0 \%$ & $0.1 \%$ & $0.0 \%$ & $0.0 \%$ & 2.08710 \\
\hline 15 & $44.9 \%$ & $0.0 \%$ & $0.0 \%$ & $0.0 \%$ & $0.0 \%$ & $0.0 \%$ & $0.0 \%$ & 2.06476 \\
\hline 16 & $0.0 \%$ & $49.2 \%$ & $49.2 \%$ & $0.4 \%$ & $0.4 \%$ & $0.4 \%$ & $0.4 \%$ & 2.06751 \\
\hline 17 & $0.0 \%$ & $45.9 \%$ & $45.9 \%$ & $1.6 \%$ & $1.6 \%$ & $1.5 \%$ & $1.5 \%$ & 2.36453 \\
\hline 18 & $0.0 \%$ & $52.7 \%$ & $0.9 \%$ & $45.3 \%$ & $0.1 \%$ & $0.6 \%$ & $0.1 \%$ & 2.07132 \\
\hline 19 & $0.0 \%$ & $53.0 \%$ & $0.9 \%$ & $0.6 \%$ & $0.1 \%$ & $44.9 \%$ & $0.1 \%$ & 2.07304 \\
\hline 20 & $0.0 \%$ & $0.9 \%$ & $52.7 \%$ & $0.1 \%$ & $45.3 \%$ & $0.1 \%$ & $0.6 \%$ & 2.07128 \\
\hline 21 & $0.0 \%$ & $0.9 \%$ & $53.0 \%$ & $0.1 \%$ & $0.6 \%$ & $0.1 \%$ & $44.9 \%$ & 2.07294 \\
\hline 22 & $0.0 \%$ & $0.6 \%$ & $0.2 \%$ & $0.0 \%$ & $0.0 \%$ & $0.3 \%$ & $0.0 \%$ & 2.61928 \\
\hline 23 & $1.4 \%$ & $0.2 \%$ & $0.6 \%$ & $0.0 \%$ & $0.0 \%$ & $0.0 \%$ & $0.3 \%$ & 2.62014 \\
\hline
\end{tabular}




\section{S1.4.3 IRC Step 04}

\begin{tabular}{|c|c|c|c|c|c|c|c|c|c|}
\hline \multicolumn{4}{|c|}{1 -center FNO analysis, $\varepsilon=0.90000$} & & & & & & \\
\hline MO & $\lambda$ & \multicolumn{2}{|l|}{ Atoms } & & & & & & \\
\hline 1 & 0.99999 & \multicolumn{2}{|l|}{$\mathrm{C}_{1}$} & & & & & & \\
\hline 2 & 0.99999 & \multicolumn{2}{|l|}{$\mathrm{C}_{2}$} & & & & & & \\
\hline 3 & 0.99999 & \multicolumn{2}{|l|}{$\mathrm{C}_{5}$} & & & & & & \\
\hline 4 & 0.99999 & \multicolumn{2}{|l|}{$\mathrm{C}_{6}$} & & & & & & \\
\hline 5 & 0.99999 & \multicolumn{2}{|l|}{$\mathrm{C}_{11}$} & & & & & & \\
\hline 6 & 0.99999 & \multicolumn{2}{|l|}{$\mathrm{C}_{12}$} & & & & & & \\
\hline \multicolumn{10}{|c|}{ 2-center FNO analysis, $\varepsilon=0.90000$} \\
\hline $\mathrm{MO}$ & $\lambda$ & \multicolumn{3}{|c|}{ Atoms } & & & & & \\
\hline 7 & 0.97462 & \multicolumn{3}{|l|}{$\mathrm{C}_{1}-\mathrm{C}_{2}$} & & & & & \\
\hline 8 & 0.97483 & \multicolumn{3}{|l|}{$\mathrm{C}_{1}-\mathrm{H}_{3}$} & & & & & \\
\hline 9 & 0.98201 & \multicolumn{3}{|l|}{$\mathrm{C}_{1}-\mathrm{C}_{5}$} & & & & & \\
\hline 10 & 0.97469 & \multicolumn{3}{|l|}{$\mathrm{C}_{2}-\mathrm{H}_{4}$} & & & & & \\
\hline 11 & 0.98196 & \multicolumn{3}{|l|}{$\mathrm{C}_{2}-\mathrm{C}_{6}$} & & & & & \\
\hline 12 & 0.97511 & \multicolumn{3}{|l|}{$\mathrm{C}_{5}-\mathrm{H}_{7}$} & & & & & \\
\hline 13 & 0.98063 & \multicolumn{3}{|l|}{$\mathrm{C}_{5}-\mathrm{H}_{9}$} & & & & & \\
\hline 14 & 0.97508 & $\mathrm{C}_{6}-\mathrm{H}_{8}$ & & & & & & & \\
\hline 15 & 0.98064 & $\mathrm{C}_{6}-\mathrm{H}_{10}$ & & & & & & & \\
\hline 16 & 0.98347 & $\mathrm{C}_{11}-\mathrm{C}_{12}$ & & & & & & & \\
\hline 17 & 0.91860 & $\mathrm{C}_{11}-\mathrm{C}_{12}$ & & & & & & & \\
\hline 18 & 0.97975 & $\mathrm{C}_{11}-\mathrm{H}_{13}$ & & & & & & & \\
\hline 19 & 0.97869 & $\mathrm{C}_{11}-\mathrm{H}_{15}$ & & & & & & & \\
\hline 20 & 0.97976 & $\mathrm{C}_{12}-\mathrm{H}_{14}$ & & & & & & & \\
\hline 21 & 0.97871 & $\mathrm{C}_{12}-\mathrm{H}_{16}$ & & & & & & & \\
\hline 3 -cent & er FNO a & lalysis, $\varepsilon=$ & $=0.9000$ & & & & & & \\
\hline $\mathrm{MO}$ & $\lambda$ & Atoms & & & & & & & \\
\hline 22 & 0.94193 & $\mathrm{C}_{1}-\mathrm{C}_{2}-$ & & & & & & & \\
\hline 23 & 0.94192 & $\mathrm{C}_{1}-\mathrm{C}_{2}-$ & & & & & & & \\
\hline Locali & zation an & atoms ex & oanded & y each & $\mathrm{NO}$ & & & & \\
\hline FNO & $\mathrm{Loc}_{1}$ & $\mathrm{Loc}_{2}$ & $\mathrm{LOC}_{3}$ & $\mathrm{Loc}_{4}$ & $\mathrm{Loc}_{5}$ & $\mathrm{LOc}_{6}$ & $\mathrm{Loc}_{7}$ & $\mathrm{LOc}_{8}$ & $\mathrm{Loc}_{9}$ \\
\hline 1 & $100.0 \%$ & $0.0 \%$ & $0.0 \%$ & $0.0 \%$ & $0.0 \%$ & $0.0 \%$ & $0.0 \%$ & $0.0 \%$ & $0.0 \%$ \\
\hline 2 & $0.0 \%$ & $100.0 \%$ & $0.0 \%$ & $0.0 \%$ & $0.0 \%$ & $0.0 \%$ & $0.0 \%$ & $0.0 \%$ & $0.0 \%$ \\
\hline 3 & $0.0 \%$ & $0.0 \%$ & $0.0 \%$ & $0.0 \%$ & $100.0 \%$ & $0.0 \%$ & $0.0 \%$ & $0.0 \%$ & $0.0 \%$ \\
\hline 4 & $0.0 \%$ & $0.0 \%$ & $0.0 \%$ & $0.0 \%$ & $0.0 \%$ & $100.0 \%$ & $0.0 \%$ & $0.0 \%$ & $0.0 \%$ \\
\hline 5 & $0.0 \%$ & $0.0 \%$ & $0.0 \%$ & $0.0 \%$ & $0.0 \%$ & $0.0 \%$ & $0.0 \%$ & $0.0 \%$ & $0.0 \%$ \\
\hline 6 & $-0.0 \%$ & $0.0 \%$ & $0.0 \%$ & $0.0 \%$ & $0.0 \%$ & $0.0 \%$ & $0.0 \%$ & $0.0 \%$ & $0.0 \%$ \\
\hline 7 & $48.7 \%$ & $48.7 \%$ & $0.5 \%$ & $0.5 \%$ & $0.6 \%$ & $0.6 \%$ & $0.1 \%$ & $0.1 \%$ & $0.1 \%$ \\
\hline 8 & $51.8 \%$ & $0.9 \%$ & $45.7 \%$ & $0.2 \%$ & $1.0 \%$ & $0.1 \%$ & $0.1 \%$ & $0.0 \%$ & $0.2 \%$ \\
\hline 9 & $49.1 \%$ & $0.4 \%$ & $0.4 \%$ & $0.1 \%$ & $49.0 \%$ & $0.1 \%$ & $0.4 \%$ & $0.0 \%$ & $0.4 \%$ \\
\hline 10 & $0.9 \%$ & $51.8 \%$ & $0.2 \%$ & $45.7 \%$ & $0.1 \%$ & $1.0 \%$ & $0.0 \%$ & $0.1 \%$ & $0.0 \%$ \\
\hline 11 & $0.4 \%$ & $49.1 \%$ & $0.1 \%$ & $0.4 \%$ & $0.1 \%$ & $49.0 \%$ & $0.0 \%$ & $0.4 \%$ & $0.0 \%$ \\
\hline 12 & $0.8 \%$ & $0.1 \%$ & $0.1 \%$ & $0.0 \%$ & $53.0 \%$ & $0.1 \%$ & $44.5 \%$ & $0.3 \%$ & $0.6 \%$ \\
\hline 13 & $0.9 \%$ & $0.1 \%$ & $0.2 \%$ & $0.0 \%$ & $53.1 \%$ & $0.0 \%$ & $0.6 \%$ & $0.0 \%$ & $44.9 \%$ \\
\hline 14 & $0.1 \%$ & $0.8 \%$ & $0.0 \%$ & $0.1 \%$ & $0.1 \%$ & $53.0 \%$ & $0.3 \%$ & $44.5 \%$ & $0.0 \%$ \\
\hline 15 & $0.1 \%$ & $0.9 \%$ & $0.0 \%$ & $0.2 \%$ & $0.0 \%$ & $53.1 \%$ & $0.0 \%$ & $0.6 \%$ & $0.0 \%$ \\
\hline 16 & $0.0 \%$ & $0.0 \%$ & $0.0 \%$ & $0.0 \%$ & $0.0 \%$ & $0.0 \%$ & $0.0 \%$ & $0.0 \%$ & $0.0 \%$ \\
\hline 17 & $0.3 \%$ & $0.3 \%$ & $0.0 \%$ & $0.0 \%$ & $0.6 \%$ & $0.6 \%$ & $0.3 \%$ & $0.3 \%$ & $0.0 \%$ \\
\hline 18 & $0.0 \%$ & $0.0 \%$ & $0.0 \%$ & $0.0 \%$ & $0.1 \%$ & $0.0 \%$ & $0.1 \%$ & $0.0 \%$ & $0.0 \%$ \\
\hline 19 & $0.1 \%$ & $0.0 \%$ & $0.0 \%$ & $0.0 \%$ & $0.2 \%$ & $0.0 \%$ & $0.0 \%$ & $0.0 \%$ & $0.0 \%$ \\
\hline 20 & $0.0 \%$ & $0.0 \%$ & $0.0 \%$ & $0.0 \%$ & $0.0 \%$ & $0.1 \%$ & $0.0 \%$ & $0.1 \%$ & $0.0 \%$ \\
\hline 21 & $0.0 \%$ & $0.1 \%$ & $0.0 \%$ & $0.0 \%$ & $0.0 \%$ & $0.2 \%$ & $0.0 \%$ & $0.0 \%$ & $0.0 \%$ \\
\hline 22 & $46.0 \%$ & $7.9 \%$ & $1.6 \%$ & $0.2 \%$ & $40.1 \%$ & $0.3 \%$ & $1.2 \%$ & $0.0 \%$ & $1.4 \%$ \\
\hline 23 & $7.9 \%$ & $46.0 \%$ & $0.2 \%$ & $1.6 \%$ & $0.3 \%$ & $40.1 \%$ & $0.0 \%$ & $1.2 \%$ & $0.0 \%$ \\
\hline
\end{tabular}




\begin{tabular}{crrrrrrrr}
\hline \multicolumn{2}{c}{ localization and atoms expanded by each FNO } \\
\hline FNO & Loc $_{10}$ & Loc $_{11}$ & Loc $_{12}$ & Loc $_{13}$ & Loc $_{14}$ & Loc $_{15}$ & Loc $_{16}$ & $n_{\text {eff }}$ \\
\hline 1 & $0.0 \%$ & $0.0 \%$ & $0.0 \%$ & $0.0 \%$ & $0.0 \%$ & $0.0 \%$ & $0.0 \%$ & 1.00002 \\
2 & $0.0 \%$ & $0.0 \%$ & $0.0 \%$ & $0.0 \%$ & $0.0 \%$ & $0.0 \%$ & $0.0 \%$ & 1.00002 \\
3 & $0.0 \%$ & $0.0 \%$ & $0.0 \%$ & $0.0 \%$ & $0.0 \%$ & $0.0 \%$ & $0.0 \%$ & 1.00002 \\
4 & $0.0 \%$ & $0.0 \%$ & $0.0 \%$ & $0.0 \%$ & $0.0 \%$ & $0.0 \%$ & $0.0 \%$ & 1.00002 \\
5 & $0.0 \%$ & $100.0 \%$ & $0.0 \%$ & $0.0 \%$ & $0.0 \%$ & $0.0 \%$ & $0.0 \%$ & 1.00002 \\
6 & $0.0 \%$ & $0.0 \%$ & $100.0 \%$ & $0.0 \%$ & $0.0 \%$ & $0.0 \%$ & $0.0 \%$ & 1.00002 \\
7 & $0.1 \%$ & $0.0 \%$ & $0.0 \%$ & $0.0 \%$ & $0.0 \%$ & $0.0 \%$ & $0.0 \%$ & 2.10527 \\
8 & $0.0 \%$ & $0.0 \%$ & $0.0 \%$ & $0.0 \%$ & $0.0 \%$ & $0.0 \%$ & $0.0 \%$ & 2.09566 \\
9 & $0.0 \%$ & $0.0 \%$ & $0.0 \%$ & $0.0 \%$ & $0.0 \%$ & $0.0 \%$ & $0.0 \%$ & 2.07420 \\
10 & $0.2 \%$ & $0.0 \%$ & $0.0 \%$ & $0.0 \%$ & $0.0 \%$ & $0.0 \%$ & $0.0 \%$ & 2.09626 \\
11 & $0.4 \%$ & $0.0 \%$ & $0.0 \%$ & $0.0 \%$ & $0.0 \%$ & $0.0 \%$ & $0.0 \%$ & 2.07426 \\
12 & $0.0 \%$ & $0.2 \%$ & $0.0 \%$ & $0.1 \%$ & $0.0 \%$ & $0.0 \%$ & $0.0 \%$ & 2.08903 \\
13 & $0.0 \%$ & $0.0 \%$ & $0.0 \%$ & $0.0 \%$ & $0.0 \%$ & $0.0 \%$ & $0.0 \%$ & 2.06505 \\
14 & $0.6 \%$ & $0.0 \%$ & $0.2 \%$ & $0.0 \%$ & $0.1 \%$ & $0.0 \%$ & $0.0 \%$ & 2.08950 \\
15 & $44.9 \%$ & $0.0 \%$ & $0.0 \%$ & $0.0 \%$ & $0.0 \%$ & $0.0 \%$ & $0.0 \%$ & 2.04459 \\
16 & $0.0 \%$ & $49.2 \%$ & $49.2 \%$ & $0.4 \%$ & $0.4 \%$ & $0.4 \%$ & $0.4 \%$ & 2.06771 \\
17 & $0.0 \%$ & $45.8 \%$ & $45.8 \%$ & $1.6 \%$ & $1.6 \%$ & $1.5 \%$ & $1.5 \%$ & 2.37475 \\
18 & $0.0 \%$ & $52.6 \%$ & $0.9 \%$ & $45.3 \%$ & $0.1 \%$ & $0.6 \%$ & $0.1 \%$ & 2.07430 \\
19 & $0.0 \%$ & $53.0 \%$ & $0.9 \%$ & $0.6 \%$ & $0.1 \%$ & $44.9 \%$ & $0.1 \%$ & 2.07440 \\
20 & $0.0 \%$ & $0.9 \%$ & $52.6 \%$ & $0.1 \%$ & $45.3 \%$ & $0.1 \%$ & $0.6 \%$ & 2.07451 \\
21 & $0.0 \%$ & $0.9 \%$ & $53.0 \%$ & $0.1 \%$ & $0.6 \%$ & $0.1 \%$ & $44.9 \%$ & 2.07400 \\
22 & $0.0 \%$ & $0.7 \%$ & $0.2 \%$ & $0.0 \%$ & $0.0 \%$ & $0.3 \%$ & $0.0 \%$ & 2.63467 \\
23 & $1.4 \%$ & $0.2 \%$ & $0.7 \%$ & $0.0 \%$ & $0.0 \%$ & $0.0 \%$ & $0.3 \%$ & 2.63497 \\
\hline \hline
\end{tabular}

\section{S1.4.4 IRC Step 06}

\begin{tabular}{|c|c|c|}
\hline \multicolumn{3}{|c|}{1 1-center FNO analysis, $\varepsilon=0.90000$} \\
\hline $\mathrm{MO}$ & $\lambda$ & Atoms \\
\hline 1 & 0.99999 & $\mathrm{C}_{1}$ \\
\hline 2 & 0.99999 & $\mathrm{C}_{2}$ \\
\hline 3 & 0.99999 & $\mathrm{C}_{5}$ \\
\hline 4 & 0.99999 & $\mathrm{C}_{6}$ \\
\hline 5 & 0.99999 & $\mathrm{C}_{11}$ \\
\hline 6 & 0.99999 & $\mathrm{C}_{12}$ \\
\hline \multicolumn{3}{|c|}{ 2-center FNO analysis, $\varepsilon=0.90000$} \\
\hline MO & $\lambda$ & Atoms \\
\hline 7 & 0.97446 & $\mathrm{C}_{1}-\mathrm{C}_{2}$ \\
\hline 8 & 0.97478 & $\mathrm{C}_{1}-\mathrm{H}_{3}$ \\
\hline 9 & 0.98173 & $\mathrm{C}_{1}-\mathrm{C}_{5}$ \\
\hline 10 & 0.97472 & $\mathrm{C}_{2}-\mathrm{H}_{4}$ \\
\hline 11 & 0.98181 & $\mathrm{C}_{2}-\mathrm{C}_{6}$ \\
\hline 12 & 0.97388 & $\mathrm{C}_{5}-\mathrm{H}_{7}$ \\
\hline 13 & 0.98055 & $\mathrm{C}_{5}-\mathrm{H}_{9}$ \\
\hline 14 & 0.97388 & $\mathrm{C}_{6}-\mathrm{H}_{8}$ \\
\hline 15 & 0.98053 & $\mathrm{C}_{6}-\mathrm{H}_{10}$ \\
\hline 16 & 0.98328 & $\mathrm{C}_{11}-\mathrm{C}_{12}$ \\
\hline 17 & 0.91386 & $\mathrm{C}_{11}-\mathrm{C}_{12}$ \\
\hline 18 & 0.97845 & $\mathrm{C}_{11}-\mathrm{H}_{13}$ \\
\hline 19 & 0.97798 & $\mathrm{C}_{11}-\mathrm{H}_{15}$ \\
\hline 20 & 0.97844 & $\mathrm{C}_{12}-\mathrm{H}_{14}$ \\
\hline 21 & 0.97801 & $\mathrm{C}_{12}-\mathrm{H}_{16}$ \\
\hline \multicolumn{3}{|c|}{3 -center FNO analysis, $\varepsilon=0.90000$} \\
\hline $\mathrm{MO}$ & $\lambda$ & Atoms \\
\hline 22 & 0.93882 & $\mathrm{C}_{1}-\mathrm{C}_{2}-\mathrm{C}_{5}$ \\
\hline 23 & 0.93881 & $\mathrm{C}_{1}-\mathrm{C}_{2}-\mathrm{C}_{6}$ \\
\hline
\end{tabular}




\begin{tabular}{|c|c|c|c|c|c|c|c|c|c|}
\hline \multicolumn{10}{|c|}{ Localization and atoms expanded by each FNO } \\
\hline FNO & $\mathrm{Loc}_{1}$ & $\mathrm{Loc}_{2}$ & $\mathrm{LOC}_{3}$ & $\mathrm{Loc}_{4}$ & $\mathrm{Loc}_{5}$ & $\mathrm{Loc}_{6}$ & $\mathrm{Loc}_{7}$ & $\mathrm{Loc}_{8}$ & $\mathrm{Loc}_{9}$ \\
\hline 1 & $100.0 \%$ & $0.0 \%$ & $0.0 \%$ & $0.0 \%$ & $0.0 \%$ & $0.0 \%$ & $0.0 \%$ & $0.0 \%$ & $0.0 \%$ \\
\hline 2 & $0.0 \%$ & $100.0 \%$ & $0.0 \%$ & $0.0 \%$ & $0.0 \%$ & $0.0 \%$ & $0.0 \%$ & $0.0 \%$ & $0.0 \%$ \\
\hline 3 & $0.0 \%$ & $0.0 \%$ & $0.0 \%$ & $0.0 \%$ & $100.0 \%$ & $0.0 \%$ & $0.0 \%$ & $0.0 \%$ & $0.0 \%$ \\
\hline 4 & $0.0 \%$ & $0.0 \%$ & $0.0 \%$ & $0.0 \%$ & $0.0 \%$ & $100.0 \%$ & $0.0 \%$ & $0.0 \%$ & $0.0 \%$ \\
\hline 5 & $0.0 \%$ & $0.0 \%$ & $0.0 \%$ & $0.0 \%$ & $0.0 \%$ & $0.0 \%$ & $0.0 \%$ & $0.0 \%$ & $0.0 \%$ \\
\hline 6 & $0.0 \%$ & $0.0 \%$ & $0.0 \%$ & $0.0 \%$ & $0.0 \%$ & $0.0 \%$ & $0.0 \%$ & $0.0 \%$ & $0.0 \%$ \\
\hline 7 & $48.7 \%$ & $48.7 \%$ & $0.5 \%$ & $0.5 \%$ & $0.6 \%$ & $0.6 \%$ & $0.1 \%$ & $0.1 \%$ & $0.1 \%$ \\
\hline 8 & $51.8 \%$ & $0.9 \%$ & $45.7 \%$ & $0.2 \%$ & $1.0 \%$ & $0.1 \%$ & $0.1 \%$ & $0.0 \%$ & $0.2 \%$ \\
\hline 9 & $49.1 \%$ & $0.4 \%$ & $0.4 \%$ & $0.1 \%$ & $49.1 \%$ & $0.1 \%$ & $0.4 \%$ & $0.0 \%$ & $0.4 \%$ \\
\hline 10 & $0.9 \%$ & $51.8 \%$ & $0.2 \%$ & $45.7 \%$ & $0.1 \%$ & $1.0 \%$ & $0.0 \%$ & $0.1 \%$ & $0.0 \%$ \\
\hline 11 & $0.4 \%$ & $49.1 \%$ & $0.1 \%$ & $0.4 \%$ & $0.1 \%$ & $49.1 \%$ & $0.0 \%$ & $0.4 \%$ & $0.0 \%$ \\
\hline 12 & $0.8 \%$ & $0.1 \%$ & $0.1 \%$ & $0.0 \%$ & $53.0 \%$ & $0.1 \%$ & $44.4 \%$ & $0.3 \%$ & $0.6 \%$ \\
\hline 13 & $0.9 \%$ & $0.1 \%$ & $0.2 \%$ & $0.0 \%$ & $53.1 \%$ & $0.0 \%$ & $0.6 \%$ & $0.0 \%$ & $44.9 \%$ \\
\hline 14 & $0.1 \%$ & $0.8 \%$ & $0.0 \%$ & $0.1 \%$ & $0.1 \%$ & $53.0 \%$ & $0.3 \%$ & $44.4 \%$ & $0.0 \%$ \\
\hline 15 & $0.1 \%$ & $0.9 \%$ & $0.0 \%$ & $0.2 \%$ & $0.0 \%$ & $53.1 \%$ & $0.0 \%$ & $0.6 \%$ & $0.0 \%$ \\
\hline 16 & $0.0 \%$ & $0.0 \%$ & $0.0 \%$ & $0.0 \%$ & $0.0 \%$ & $0.0 \%$ & $0.0 \%$ & $0.0 \%$ & $0.0 \%$ \\
\hline 17 & $0.4 \%$ & $0.3 \%$ & $0.0 \%$ & $0.0 \%$ & $0.6 \%$ & $0.6 \%$ & $0.3 \%$ & $0.3 \%$ & $0.0 \%$ \\
\hline 18 & $0.0 \%$ & $0.0 \%$ & $0.0 \%$ & $0.0 \%$ & $0.2 \%$ & $0.0 \%$ & $0.2 \%$ & $0.0 \%$ & $0.0 \%$ \\
\hline 19 & $0.1 \%$ & $0.0 \%$ & $0.0 \%$ & $0.0 \%$ & $0.2 \%$ & $0.0 \%$ & $0.0 \%$ & $0.0 \%$ & $0.0 \%$ \\
\hline 20 & $0.0 \%$ & $0.0 \%$ & $0.0 \%$ & $0.0 \%$ & $0.0 \%$ & $0.2 \%$ & $0.0 \%$ & $0.2 \%$ & $0.0 \%$ \\
\hline 21 & $0.0 \%$ & $0.1 \%$ & $0.0 \%$ & $0.0 \%$ & $0.0 \%$ & $0.2 \%$ & $0.0 \%$ & $0.0 \%$ & $0.0 \%$ \\
\hline 22 & $46.0 \%$ & $8.1 \%$ & $1.6 \%$ & $0.2 \%$ & $39.8 \%$ & $0.3 \%$ & $1.2 \%$ & $0.0 \%$ & $1.4 \%$ \\
\hline 23 & $8.2 \%$ & $45.9 \%$ & $0.2 \%$ & $1.6 \%$ & $0.3 \%$ & $39.8 \%$ & $0.0 \%$ & $1.2 \%$ & $0.0 \%$ \\
\hline
\end{tabular}

\begin{tabular}{crrrrrrrr}
\hline \hline \multicolumn{6}{|c}{ Localization and atoms expanded by each FNO } \\
\hline FNO & Loc $_{10}$ & Loc $_{11}$ & Loc $_{12}$ & Loc $_{13}$ & Loc $_{14}$ & Loc $_{15}$ & Loc $_{16}$ & $n_{\text {eff }}$ \\
\hline 1 & $0.0 \%$ & $0.0 \%$ & $0.0 \%$ & $0.0 \%$ & $0.0 \%$ & $0.0 \%$ & $0.0 \%$ & 1.00002 \\
2 & $0.0 \%$ & $0.0 \%$ & $0.0 \%$ & $0.0 \%$ & $0.0 \%$ & $0.0 \%$ & $0.0 \%$ & 1.00002 \\
3 & $0.0 \%$ & $0.0 \%$ & $0.0 \%$ & $0.0 \%$ & $0.0 \%$ & $0.0 \%$ & $0.0 \%$ & 1.00002 \\
4 & $0.0 \%$ & $0.0 \%$ & $0.0 \%$ & $0.0 \%$ & $0.0 \%$ & $0.0 \%$ & $0.0 \%$ & 1.00002 \\
5 & $0.0 \%$ & 100.0 & $0.0 \%$ & $0.0 \%$ & $0.0 \%$ & $0.0 \%$ & $0.0 \%$ & 1.00002 \\
6 & $0.0 \%$ & $0.0 \%$ & $100.0 \%$ & $0.0 \%$ & $0.0 \%$ & $0.0 \%$ & $0.0 \%$ & 1.00002 \\
7 & $0.1 \%$ & $0.0 \%$ & $0.0 \%$ & $0.0 \%$ & $0.0 \%$ & $0.0 \%$ & $0.0 \%$ & 2.10565 \\
8 & $0.0 \%$ & $0.0 \%$ & $0.0 \%$ & $0.0 \%$ & $0.0 \%$ & $0.0 \%$ & $0.0 \%$ & 2.09572 \\
9 & $0.0 \%$ & $0.0 \%$ & $0.0 \%$ & $0.0 \%$ & $0.0 \%$ & $0.0 \%$ & $0.0 \%$ & 2.07485 \\
10 & $0.2 \%$ & $0.0 \%$ & $0.0 \%$ & $0.0 \%$ & $0.0 \%$ & $0.0 \%$ & $0.0 \%$ & 2.09599 \\
11 & $0.4 \%$ & $0.0 \%$ & $0.0 \%$ & $0.0 \%$ & $0.0 \%$ & $0.0 \%$ & $0.0 \%$ & 2.07451 \\
12 & $0.0 \%$ & $0.3 \%$ & $0.0 \%$ & $0.1 \%$ & $0.0 \%$ & $0.0 \%$ & $0.0 \%$ & 2.02040 \\
13 & $0.0 \%$ & $0.0 \%$ & $0.0 \%$ & $0.0 \%$ & $0.0 \%$ & $0.0 \%$ & $0.0 \%$ & 2.06526 \\
14 & $0.6 \%$ & $0.0 \%$ & $0.3 \%$ & $0.0 \%$ & $0.1 \%$ & $0.0 \%$ & $0.0 \%$ & 2.09208 \\
15 & $444.9 \%$ & $0.0 \%$ & $0.0 \%$ & $0.0 \%$ & $0.0 \%$ & $0.0 \%$ & $0.0 \%$ & 2.06532 \\
16 & $0.0 \%$ & $49.2 \%$ & $49.2 \%$ & $0.4 \%$ & $0.4 \%$ & $0.4 \%$ & $0.4 \%$ & 2.06832 \\
17 & $0.0 \%$ & $45.7 \%$ & $45.7 \%$ & $1.6 \%$ & $1.6 \%$ & $1.5 \%$ & $1.5 \%$ & 2.38888 \\
18 & $0.0 \%$ & $52.5 \%$ & $0.9 \%$ & $45.3 \%$ & $0.1 \%$ & $0.6 \%$ & $0.1 \%$ & 2.07290 \\
19 & $0.0 \%$ & $52.9 \%$ & $0.9 \%$ & $0.6 \%$ & $0.1 \%$ & $44.9 \%$ & $0.1 \%$ & 2.07637 \\
20 & $0.0 \%$ & $0.9 \%$ & $52.5 \%$ & $0.1 \%$ & $45.3 \%$ & $0.1 \%$ & $0.6 \%$ & 2.07731 \\
21 & $0.0 \%$ & $0.9 \%$ & $52.9 \%$ & $0.1 \%$ & $0.6 \%$ & $0.1 \%$ & $44.9 \%$ & 2.07627 \\
22 & $0.0 \%$ & $0.8 \%$ & $0.3 \%$ & $0.1 \%$ & $0.0 \%$ & $0.3 \%$ & $0.0 \%$ & 2.65435 \\
23 & $1.4 \%$ & $0.3 \%$ & $0.8 \%$ & $0.0 \%$ & $0.1 \%$ & $0.0 \%$ & $0.3 \%$ & 2.65441 \\
\hline \hline
\end{tabular}




\section{S1.4.5 IRC Step 08}

\begin{tabular}{|c|c|c|c|c|c|c|c|c|c|}
\hline \multicolumn{4}{|c|}{ 1-center FNO analysis, $\varepsilon=0.90000$} & & & & & & \\
\hline MO & $\lambda$ & \multicolumn{2}{|l|}{ Atoms } & & & & & & \\
\hline 1 & 0.99999 & \multicolumn{2}{|l|}{$\mathrm{C}_{1}$} & & & & & & \\
\hline 2 & 0.99999 & \multicolumn{2}{|l|}{$\mathrm{C}_{2}$} & & & & & & \\
\hline 3 & 0.99999 & \multicolumn{2}{|l|}{$\mathrm{C}_{5}$} & & & & & & \\
\hline 4 & 0.99999 & \multicolumn{2}{|l|}{$\mathrm{C}_{6}$} & & & & & & \\
\hline 5 & 0.99999 & \multicolumn{2}{|l|}{$\mathrm{C}_{11}$} & & & & & & \\
\hline 6 & 0.99999 & \multicolumn{2}{|l|}{$\mathrm{C}_{12}$} & & & & & & \\
\hline \multicolumn{10}{|c|}{ 2-center FNO analysis, $\varepsilon=0.90000$} \\
\hline $\mathrm{MO}$ & $\lambda$ & \multicolumn{3}{|l|}{ Atoms } & & & & & \\
\hline 7 & 0.97434 & \multicolumn{3}{|l|}{$\mathrm{C}_{1}-\mathrm{C}_{2}$} & & & & & \\
\hline 8 & 0.97484 & \multicolumn{3}{|l|}{$\mathrm{C}_{1}-\mathrm{H}_{3}$} & & & & & \\
\hline 9 & 0.98152 & \multicolumn{3}{|l|}{$\mathrm{C}_{1}-\mathrm{C}_{5}$} & & & & & \\
\hline 10 & 0.97478 & \multicolumn{3}{|l|}{$\mathrm{C}_{2}-\mathrm{H}_{4}$} & & & & & \\
\hline 11 & 0.98148 & \multicolumn{3}{|l|}{$\mathrm{C}_{2}-\mathrm{C}_{6}$} & & & & & \\
\hline 12 & 0.97277 & \multicolumn{3}{|l|}{$\mathrm{C}_{5}-\mathrm{H}_{7}$} & & & & & \\
\hline 13 & 0.98042 & \multicolumn{3}{|l|}{$\mathrm{C}_{5}-\mathrm{H}_{9}$} & & & & & \\
\hline 14 & 0.97276 & $\mathrm{C}_{6}-\mathrm{H}_{8}$ & & & & & & & \\
\hline 15 & 0.98042 & $\mathrm{C}_{6}-\mathrm{H}_{10}$ & & & & & & & \\
\hline 16 & 0.98298 & $\mathrm{C}_{11}-\mathrm{C}_{12}$ & & & & & & & \\
\hline 17 & 0.91032 & $\mathrm{C}_{11}-\mathrm{C}_{12}$ & & & & & & & \\
\hline 18 & 0.97772 & $\mathrm{C}_{11}-\mathrm{H}_{13}$ & & & & & & & \\
\hline 19 & 0.97729 & $\mathrm{C}_{11}-\mathrm{H}_{15}$ & & & & & & & \\
\hline 20 & 0.97771 & $\mathrm{C}_{12}-\mathrm{H}_{14}$ & & & & & & & \\
\hline 21 & 0.97728 & $\mathrm{C}_{12}-\mathrm{H}_{16}$ & & & & & & & \\
\hline 3 -cent & er FNO a & lalysis, $\varepsilon=$ & $=0.90000$ & & & & & & \\
\hline $\mathrm{MO}$ & $\lambda$ & Atoms & & & & & & & \\
\hline 22 & 0.93703 & $\mathrm{C}_{1}-\mathrm{C}_{2}-$ & & & & & & & \\
\hline 23 & 0.93703 & $\mathrm{C}_{1}-\mathrm{C}_{2}-$ & & & & & & & \\
\hline Locali & zation an & atoms ex & panded 1 & y each & $\mathrm{NO}$ & & & & \\
\hline FNO & $\mathrm{Loc}_{1}$ & $\mathrm{Loc}_{2}$ & $\mathrm{Loc}_{3}$ & $\mathrm{Loc}_{4}$ & $\mathrm{Loc}_{5}$ & $\mathrm{Loc}_{6}$ & $\mathrm{Loc}_{7}$ & $\mathrm{LOc}_{8}$ & $\mathrm{Loc}_{9}$ \\
\hline 1 & $100.0 \%$ & $0.0 \%$ & $0.0 \%$ & $0.0 \%$ & $0.0 \%$ & $0.0 \%$ & $0.0 \%$ & $0.0 \%$ & $0.0 \%$ \\
\hline 2 & $0.0 \%$ & $100.0 \%$ & $0.0 \%$ & $0.0 \%$ & $0.0 \%$ & $0.0 \%$ & $0.0 \%$ & $0.0 \%$ & $0.0 \%$ \\
\hline 3 & $0.0 \%$ & $0.0 \%$ & $0.0 \%$ & $0.0 \%$ & $100.0 \%$ & $0.0 \%$ & $0.0 \%$ & $0.0 \%$ & $0.0 \%$ \\
\hline 4 & $0.0 \%$ & $0.0 \%$ & $0.0 \%$ & $0.0 \%$ & $0.0 \%$ & $100.0 \%$ & $0.0 \%$ & $0.0 \%$ & $0.0 \%$ \\
\hline 5 & $0.0 \%$ & $0.0 \%$ & $0.0 \%$ & $0.0 \%$ & $0.0 \%$ & $0.0 \%$ & $0.0 \%$ & $0.0 \%$ & $0.0 \%$ \\
\hline 6 & $0.0 \%$ & $0.0 \%$ & $0.0 \%$ & $0.0 \%$ & $0.0 \%$ & $0.0 \%$ & $0.0 \%$ & $0.0 \%$ & $0.0 \%$ \\
\hline 7 & $48.7 \%$ & $48.7 \%$ & $0.5 \%$ & $0.5 \%$ & $0.6 \%$ & $0.6 \%$ & $0.1 \%$ & $0.1 \%$ & $0.1 \%$ \\
\hline 8 & $51.8 \%$ & $0.9 \%$ & $45.7 \%$ & $0.2 \%$ & $1.0 \%$ & $0.1 \%$ & $0.1 \%$ & $0.0 \%$ & $0.2 \%$ \\
\hline 9 & $49.0 \%$ & $0.5 \%$ & $0.4 \%$ & $0.1 \%$ & $49.1 \%$ & $0.1 \%$ & $0.4 \%$ & $0.0 \%$ & $0.4 \%$ \\
\hline 10 & $0.9 \%$ & $51.8 \%$ & $0.2 \%$ & $45.7 \%$ & $0.1 \%$ & $1.0 \%$ & $0.0 \%$ & $0.1 \%$ & $0.0 \%$ \\
\hline 11 & $0.5 \%$ & $49.0 \%$ & $0.1 \%$ & $0.4 \%$ & $0.1 \%$ & $49.1 \%$ & $0.0 \%$ & $0.4 \%$ & $0.0 \%$ \\
\hline 12 & $0.8 \%$ & $0.1 \%$ & $0.1 \%$ & $0.0 \%$ & $52.9 \%$ & $0.1 \%$ & $44.4 \%$ & $0.3 \%$ & $0.6 \%$ \\
\hline 13 & $0.9 \%$ & $0.1 \%$ & $0.2 \%$ & $0.0 \%$ & $53.1 \%$ & $0.0 \%$ & $0.6 \%$ & $0.0 \%$ & $45.0 \%$ \\
\hline 14 & $0.1 \%$ & $0.8 \%$ & $0.0 \%$ & $0.1 \%$ & $0.1 \%$ & $52.9 \%$ & $0.3 \%$ & $44.4 \%$ & $0.0 \%$ \\
\hline 15 & $0.1 \%$ & $0.9 \%$ & $0.0 \%$ & $0.2 \%$ & $0.0 \%$ & $53.1 \%$ & $0.0 \%$ & $0.6 \%$ & $0.0 \%$ \\
\hline 16 & $0.0 \%$ & $0.0 \%$ & $0.0 \%$ & $0.0 \%$ & $0.0 \%$ & $0.0 \%$ & $0.0 \%$ & $0.0 \%$ & $0.0 \%$ \\
\hline 17 & $0.4 \%$ & $0.4 \%$ & $0.0 \%$ & $0.0 \%$ & $0.8 \%$ & $0.8 \%$ & $0.3 \%$ & $0.3 \%$ & $0.0 \%$ \\
\hline 18 & $0.0 \%$ & $0.0 \%$ & $0.0 \%$ & $0.0 \%$ & $0.2 \%$ & $0.0 \%$ & $0.2 \%$ & $0.0 \%$ & $0.0 \%$ \\
\hline 19 & $0.1 \%$ & $0.0 \%$ & $0.0 \%$ & $0.0 \%$ & $0.3 \%$ & $0.0 \%$ & $0.0 \%$ & $0.0 \%$ & $0.0 \%$ \\
\hline 20 & $-0.0 \%$ & $0.0 \%$ & $0.0 \%$ & $0.0 \%$ & $0.0 \%$ & $0.2 \%$ & $0.0 \%$ & $0.2 \%$ & $0.0 \%$ \\
\hline 21 & $0.0 \%$ & $0.1 \%$ & $0.0 \%$ & $0.0 \%$ & $0.0 \%$ & $0.3 \%$ & $0.0 \%$ & $0.0 \%$ & $0.0 \%$ \\
\hline 22 & $45.9 \%$ & $8.5 \%$ & $1.6 \%$ & $0.2 \%$ & $39.3 \%$ & $0.3 \%$ & $1.1 \%$ & $0.0 \%$ & $1.3 \%$ \\
\hline 23 & $8.5 \%$ & $45.9 \%$ & $0.2 \%$ & $1.6 \%$ & $0.3 \%$ & $39.3 \%$ & $0.0 \%$ & $1.1 \%$ & $0.0 \%$ \\
\hline
\end{tabular}




\begin{tabular}{crrrrrrrr}
\hline \multicolumn{2}{l}{ localization and atoms expanded by each FNO } \\
\hline FNO & Loc $_{10}$ & Loc $_{11}$ & Loc $_{12}$ & Loc $_{13}$ & Loc $_{14}$ & Loc $_{15}$ & Loc $_{16}$ & $n_{\text {eff }}$ \\
\hline 1 & $0.0 \%$ & $0.0 \%$ & $0.0 \%$ & $0.0 \%$ & $0.0 \%$ & $0.0 \%$ & $0.0 \%$ & 1.00002 \\
2 & $0.0 \%$ & $0.0 \%$ & $0.0 \%$ & $0.0 \%$ & $0.0 \%$ & $0.0 \%$ & $0.0 \%$ & 1.00002 \\
3 & $0.0 \%$ & $0.0 \%$ & $0.0 \%$ & $0.0 \%$ & $0.0 \%$ & $0.0 \%$ & $0.0 \%$ & 1.00002 \\
4 & $0.0 \%$ & $0.0 \%$ & $0.0 \%$ & $0.0 \%$ & $0.0 \%$ & $0.0 \%$ & $0.0 \%$ & 1.00002 \\
5 & $0.0 \%$ & $100.0 \%$ & $0.0 \%$ & $0.0 \%$ & $0.0 \%$ & $0.0 \%$ & $0.0 \%$ & 1.00002 \\
6 & $0.0 \%$ & $0.0 \%$ & $100.0 \%$ & $0.0 \%$ & $0.0 \%$ & $0.0 \%$ & $0.0 \%$ & 1.00002 \\
7 & $0.1 \%$ & $0.0 \%$ & $0.0 \%$ & $0.0 \%$ & $0.0 \%$ & $0.0 \%$ & $0.0 \%$ & 2.10618 \\
8 & $0.0 \%$ & $0.0 \%$ & $0.0 \%$ & $0.0 \%$ & $0.0 \%$ & $0.0 \%$ & $0.0 \%$ & 2.09547 \\
9 & $0.0 \%$ & $0.0 \%$ & $0.0 \%$ & $0.0 \%$ & $0.0 \%$ & $0.0 \%$ & $0.0 \%$ & 2.07570 \\
10 & $0.2 \%$ & $0.0 \%$ & $0.0 \%$ & $0.0 \%$ & $0.0 \%$ & $0.0 \%$ & $0.0 \%$ & 2.09573 \\
11 & $0.4 \%$ & $0.0 \%$ & $0.0 \%$ & $0.0 \%$ & $0.0 \%$ & $0.0 \%$ & $0.0 \%$ & 2.07587 \\
12 & $0.0 \%$ & $0.3 \%$ & $0.1 \%$ & $0.2 \%$ & $0.0 \%$ & $0.0 \%$ & $0.0 \%$ & 2.09668 \\
13 & $0.0 \%$ & $0.0 \%$ & $0.0 \%$ & $0.0 \%$ & $0.0 \%$ & $0.0 \%$ & $0.0 \%$ & 2.06592 \\
14 & $0.6 \%$ & $0.1 \%$ & $0.3 \%$ & $0.0 \%$ & $0.2 \%$ & $0.0 \%$ & $0.0 \%$ & 2.09677 \\
15 & $45.0 \%$ & $0.0 \%$ & $0.0 \%$ & $0.0 \%$ & $0.0 \%$ & $0.0 \%$ & $0.0 \%$ & 2.06595 \\
16 & $0.0 \%$ & $49.1 \%$ & $49.2 \%$ & $0.4 \%$ & $0.4 \%$ & $0.4 \%$ & $0.4 \%$ & 2.06960 \\
17 & $0.0 \%$ & $45.5 \%$ & $45.5 \%$ & $1.5 \%$ & $1.5 \%$ & $1.4 \%$ & $1.4 \%$ & 2.40733 \\
18 & $0.0 \%$ & $52.4 \%$ & $0.9 \%$ & $45.3 \%$ & $0.1 \%$ & $0.6 \%$ & $0.1 \%$ & 2.08058 \\
19 & $0.0 \%$ & $52.9 \%$ & $0.9 \%$ & $0.6 \%$ & $0.1 \%$ & $44.9 \%$ & $0.1 \%$ & 2.07945 \\
20 & $0.0 \%$ & $0.9 \%$ & $52.4 \%$ & $0.1 \%$ & $45.3 \%$ & $0.1 \%$ & $0.6 \%$ & 2.08060 \\
21 & $0.0 \%$ & $0.9 \%$ & $52.9 \%$ & $0.1 \%$ & $0.6 \%$ & $0.1 \%$ & $44.9 \%$ & 2.07947 \\
22 & $0.0 \%$ & $0.9 \%$ & $0.3 \%$ & $0.1 \%$ & $0.0 \%$ & $0.3 \%$ & $0.0 \%$ & 2.67787 \\
23 & $1.3 \%$ & $0.3 \%$ & $0.9 \%$ & $0.0 \%$ & $0.1 \%$ & $0.0 \%$ & $0.3 \%$ & 2.67853 \\
\hline \hline
\end{tabular}

\section{S1.4.6 IRC Step 10}

\begin{tabular}{|c|c|c|}
\hline \multicolumn{3}{|c|}{1 1-center FNO analysis, $\varepsilon=0.90000$} \\
\hline $\mathrm{MO}$ & $\lambda$ & Atoms \\
\hline 1 & 0.99999 & $\mathrm{C}_{1}$ \\
\hline 2 & 0.99999 & $\mathrm{C}_{2}$ \\
\hline 3 & 0.99999 & $\mathrm{C}_{5}$ \\
\hline 4 & 0.99999 & $\mathrm{C}_{6}$ \\
\hline 5 & 0.99999 & $\mathrm{C}_{11}$ \\
\hline 6 & 0.99999 & $\mathrm{C}_{12}$ \\
\hline \multicolumn{3}{|c|}{ 2-center FNO analysis, $\varepsilon=0.90000$} \\
\hline MO & $\lambda$ & Atoms \\
\hline 7 & 0.97418 & $\mathrm{C}_{1}-\mathrm{C}_{2}$ \\
\hline 8 & 0.97496 & $\mathrm{C}_{1}-\mathrm{H}_{3}$ \\
\hline 9 & 0.98125 & $\mathrm{C}_{1}-\mathrm{C}_{5}$ \\
\hline 10 & 0.97477 & $\mathrm{C}_{2}-\mathrm{H}_{4}$ \\
\hline 11 & 0.98124 & $\mathrm{C}_{2}-\mathrm{C}_{6}$ \\
\hline 12 & 0.97153 & $\mathrm{C}_{5}-\mathrm{H}_{7}$ \\
\hline 13 & 0.98021 & $\mathrm{C}_{5}-\mathrm{H}_{9}$ \\
\hline 14 & 0.97153 & $\mathrm{C}_{6}-\mathrm{H}_{8}$ \\
\hline 15 & 0.98020 & $\mathrm{C}_{6}-\mathrm{H}_{10}$ \\
\hline 16 & 0.98265 & $\mathrm{C}_{11}-\mathrm{C}_{12}$ \\
\hline 17 & 0.90526 & $\mathrm{C}_{11}-\mathrm{C}_{12}$ \\
\hline 18 & 0.97658 & $\mathrm{C}_{11}-\mathrm{H}_{13}$ \\
\hline 19 & 0.97664 & $\mathrm{C}_{11}-\mathrm{H}_{15}$ \\
\hline 20 & 0.97660 & $\mathrm{C}_{12}-\mathrm{H}_{14}$ \\
\hline 21 & 0.97665 & $\mathrm{C}_{12}-\mathrm{H}_{16}$ \\
\hline \multicolumn{3}{|c|}{3 -center FNO analysis, $\varepsilon=0.90000$} \\
\hline $\mathrm{MO}$ & $\lambda$ & Atoms \\
\hline 22 & 0.93485 & $\mathrm{C}_{1}-\mathrm{C}_{2}-\mathrm{C}_{5}$ \\
\hline 23 & 0.93485 & $\mathrm{C}_{1}-\mathrm{C}_{2}-\mathrm{C}_{6}$ \\
\hline
\end{tabular}




\begin{tabular}{|c|c|c|c|c|c|c|c|c|c|}
\hline \multicolumn{10}{|c|}{ Localization and atoms expanded by each FNO } \\
\hline FNO & $\mathrm{Loc}_{1}$ & $\mathrm{Loc}_{2}$ & $\mathrm{LOC}_{3}$ & $\mathrm{Loc}_{4}$ & $\mathrm{Loc}_{5}$ & $\mathrm{Loc}_{6}$ & $\mathrm{LOC}_{7}$ & $\mathrm{Loc}_{8}$ & $\mathrm{Loc}_{9}$ \\
\hline 1 & $100.0 \%$ & $0.0 \%$ & $0.0 \%$ & $0.0 \%$ & $0.0 \%$ & $0.0 \%$ & $0.0 \%$ & $0.0 \%$ & $0.0 \%$ \\
\hline 2 & $0.0 \%$ & $100.0 \%$ & $0.0 \%$ & $0.0 \%$ & $0.0 \%$ & $0.0 \%$ & $0.0 \%$ & $0.0 \%$ & $0.0 \%$ \\
\hline 3 & $0.0 \%$ & $0.0 \%$ & $0.0 \%$ & $0.0 \%$ & $100.0 \%$ & $0.0 \%$ & $0.0 \%$ & $0.0 \%$ & $0.0 \%$ \\
\hline 4 & $0.0 \%$ & $0.0 \%$ & $0.0 \%$ & $0.0 \%$ & $0.0 \%$ & $100.0 \%$ & $0.0 \%$ & $0.0 \%$ & $0.0 \%$ \\
\hline 5 & $0.0 \%$ & $0.0 \%$ & $0.0 \%$ & $0.0 \%$ & $0.0 \%$ & $0.0 \%$ & $0.0 \%$ & $0.0 \%$ & $0.0 \%$ \\
\hline 6 & $0.0 \%$ & $0.0 \%$ & $0.0 \%$ & $0.0 \%$ & $0.0 \%$ & $0.0 \%$ & $0.0 \%$ & $0.0 \%$ & $0.0 \%$ \\
\hline 7 & $48.7 \%$ & $48.7 \%$ & $0.5 \%$ & $0.5 \%$ & $0.6 \%$ & $0.6 \%$ & $0.1 \%$ & $0.1 \%$ & $0.1 \%$ \\
\hline 8 & $51.8 \%$ & $0.9 \%$ & $45.7 \%$ & $0.2 \%$ & $1.0 \%$ & $0.1 \%$ & $0.1 \%$ & $0.0 \%$ & $0.2 \%$ \\
\hline 9 & $48.9 \%$ & $0.5 \%$ & $0.4 \%$ & $0.1 \%$ & $49.2 \%$ & $0.1 \%$ & $0.4 \%$ & $0.0 \%$ & $0.4 \%$ \\
\hline 10 & $0.9 \%$ & $51.8 \%$ & $0.2 \%$ & $45.7 \%$ & $0.1 \%$ & $1.0 \%$ & $0.0 \%$ & $0.1 \%$ & $0.0 \%$ \\
\hline 11 & $0.5 \%$ & $48.9 \%$ & $0.1 \%$ & $0.4 \%$ & $0.1 \%$ & $49.2 \%$ & $0.0 \%$ & $0.4 \%$ & $0.0 \%$ \\
\hline 12 & $0.8 \%$ & $0.1 \%$ & $0.1 \%$ & $0.0 \%$ & $52.9 \%$ & $0.1 \%$ & $44.3 \%$ & $0.4 \%$ & $0.6 \%$ \\
\hline 13 & $0.9 \%$ & $0.1 \%$ & $0.2 \%$ & $0.0 \%$ & $53.1 \%$ & $0.0 \%$ & $0.6 \%$ & $0.0 \%$ & $45.0 \%$ \\
\hline 14 & $0.1 \%$ & $0.8 \%$ & $0.0 \%$ & $0.1 \%$ & $0.1 \%$ & $52.9 \%$ & $0.4 \%$ & $44.3 \%$ & $0.0 \%$ \\
\hline 15 & $0.1 \%$ & $0.9 \%$ & $0.0 \%$ & $0.2 \%$ & $0.0 \%$ & $53.1 \%$ & $0.0 \%$ & $0.6 \%$ & $0.0 \%$ \\
\hline 16 & $0.0 \%$ & $0.0 \%$ & $0.0 \%$ & $0.0 \%$ & $0.0 \%$ & $0.0 \%$ & $0.0 \%$ & $0.0 \%$ & $0.0 \%$ \\
\hline 17 & $0.5 \%$ & $0.5 \%$ & $0.0 \%$ & $0.0 \%$ & $0.9 \%$ & $0.9 \%$ & $0.3 \%$ & $0.3 \%$ & $0.0 \%$ \\
\hline 18 & $0.0 \%$ & $0.0 \%$ & $0.0 \%$ & $0.0 \%$ & $0.2 \%$ & $0.0 \%$ & $0.2 \%$ & $0.0 \%$ & $0.0 \%$ \\
\hline 19 & $0.1 \%$ & $0.0 \%$ & $0.0 \%$ & $0.0 \%$ & $0.3 \%$ & $0.0 \%$ & $0.0 \%$ & $0.0 \%$ & $0.0 \%$ \\
\hline 20 & $0.0 \%$ & $0.0 \%$ & $0.0 \%$ & $0.0 \%$ & $0.0 \%$ & $0.2 \%$ & $0.0 \%$ & $0.2 \%$ & $0.0 \%$ \\
\hline 21 & $0.0 \%$ & $0.1 \%$ & $0.0 \%$ & $0.0 \%$ & $0.0 \%$ & $0.3 \%$ & $0.0 \%$ & $0.0 \%$ & $0.0 \%$ \\
\hline 22 & $45.9 \%$ & $8.9 \%$ & $1.6 \%$ & $0.2 \%$ & $38.7 \%$ & $0.3 \%$ & $1.1 \%$ & $0.0 \%$ & $1.3 \%$ \\
\hline 23 & $8.9 \%$ & $45.9 \%$ & $0.2 \%$ & $1.6 \%$ & $0.3 \%$ & $38.7 \%$ & $0.0 \%$ & $1.1 \%$ & $0.0 \%$ \\
\hline
\end{tabular}

\begin{tabular}{crrrrrrrr}
\hline \hline \multicolumn{6}{|c}{ Localization and atoms expanded by each FNO } \\
\hline FNO & Loc $_{10}$ & Loc $_{11}$ & Loc $_{12}$ & Loc $_{13}$ & Loc $_{14}$ & Loc $_{15}$ & Loc $_{16}$ & $n_{\text {eff }}$ \\
\hline 1 & $0.0 \%$ & $0.0 \%$ & $0.0 \%$ & $0.0 \%$ & $0.0 \%$ & $0.0 \%$ & $0.0 \%$ & 1.00002 \\
2 & $0.0 \%$ & $0.0 \%$ & $0.0 \%$ & $0.0 \%$ & $0.0 \%$ & $0.0 \%$ & $0.0 \%$ & 1.00002 \\
3 & $0.0 \%$ & $0.0 \%$ & $0.0 \%$ & $0.0 \%$ & $0.0 \%$ & $0.0 \%$ & $0.0 \%$ & 1.00002 \\
4 & $0.0 \%$ & $0.0 \%$ & $0.0 \%$ & $0.0 \%$ & $0.0 \%$ & $0.0 \%$ & $0.0 \%$ & 1.00002 \\
5 & $0.0 \%$ & $100.0 \%$ & $0.0 \%$ & $0.0 \%$ & $0.0 \%$ & $0.0 \%$ & $0.0 \%$ & 1.00002 \\
6 & $0.0 \%$ & $0.0 \%$ & $100.0 \%$ & $0.0 \%$ & $0.0 \%$ & $0.0 \%$ & $0.0 \%$ & 1.00002 \\
7 & $0.1 \%$ & $0.0 \%$ & $0.0 \%$ & $0.0 \%$ & $0.0 \%$ & $0.0 \%$ & $0.0 \%$ & 2.10686 \\
8 & $0.0 \%$ & $0.0 \%$ & $0.0 \%$ & $0.0 \%$ & $0.0 \%$ & $0.0 \%$ & $0.0 \%$ & 2.09492 \\
9 & $0.0 \%$ & $0.1 \%$ & $0.0 \%$ & $0.0 \%$ & $0.0 \%$ & $0.0 \%$ & $0.0 \%$ & 2.07683 \\
10 & $0.2 \%$ & $0.0 \%$ & $0.0 \%$ & $0.0 \%$ & $0.0 \%$ & $0.0 \%$ & $0.0 \%$ & 2.09575 \\
11 & $0.4 \%$ & $0.0 \%$ & $0.1 \%$ & $0.0 \%$ & $0.0 \%$ & $0.0 \%$ & $0.0 \%$ & 2.07687 \\
12 & $0.0 \%$ & $0.4 \%$ & $0.1 \%$ & $0.2 \%$ & $0.0 \%$ & $0.0 \%$ & $0.0 \%$ & 2.10185 \\
13 & $0.0 \%$ & $0.1 \%$ & $0.0 \%$ & $0.0 \%$ & $0.0 \%$ & $0.0 \%$ & $0.0 \%$ & 2.06691 \\
14 & $0.6 \%$ & $0.1 \%$ & $0.4 \%$ & $0.0 \%$ & $0.2 \%$ & $0.0 \%$ & $0.0 \%$ & 2.10186 \\
15 & $45.0 \%$ & $0.0 \%$ & $0.1 \%$ & $0.0 \%$ & $0.0 \%$ & $0.0 \%$ & $0.0 \%$ & 2.06699 \\
16 & $0.0 \%$ & $49.1 \%$ & $49.1 \%$ & $0.4 \%$ & $0.4 \%$ & $0.4 \%$ & $0.4 \%$ & 2.07096 \\
17 & $0.0 \%$ & $45.3 \%$ & $45.3 \%$ & $1.5 \%$ & $1.5 \%$ & $1.4 \%$ & $1.4 \%$ & 2.43391 \\
18 & $0.0 \%$ & $52.3 \%$ & $0.9 \%$ & $45.3 \%$ & $0.1 \%$ & $0.6 \%$ & $0.1 \%$ & 2.08570 \\
19 & $0.0 \%$ & $52.8 \%$ & $0.9 \%$ & $0.6 \%$ & $0.1 \%$ & $44.8 \%$ & $0.1 \%$ & 2.08225 \\
20 & $0.0 \%$ & $0.9 \%$ & $52.3 \%$ & $0.1 \%$ & $45.3 \%$ & $0.1 \%$ & $0.6 \%$ & 2.08562 \\
21 & $0.0 \%$ & $0.9 \%$ & $52.8 \%$ & $0.1 \%$ & $0.6 \%$ & $0.1 \%$ & $44.8 \%$ & 2.08223 \\
22 & $0.0 \%$ & $1.0 \%$ & $0.4 \%$ & $0.1 \%$ & $0.0 \%$ & $0.4 \%$ & $0.0 \%$ & 2.70968 \\
23 & $1.3 \%$ & $0.4 \%$ & $1.0 \%$ & $0.0 \%$ & $0.1 \%$ & $0.0 \%$ & $0.4 \%$ & 2.71011 \\
\hline \hline & & & & & & & & \\
\end{tabular}




\section{S1.4.7 IRC Step 12}

\begin{tabular}{|c|c|c|c|c|c|c|c|c|c|}
\hline \multicolumn{4}{|c|}{ 1-center FNO analysis, $\varepsilon=0.90000$} & & & & & & \\
\hline MO & $\lambda$ & \multicolumn{2}{|l|}{ Atoms } & & & & & & \\
\hline 1 & 0.99999 & \multicolumn{2}{|l|}{$\mathrm{C}_{1}$} & & & & & & \\
\hline 2 & 0.99999 & \multicolumn{2}{|l|}{$\mathrm{C}_{2}$} & & & & & & \\
\hline 3 & 0.99999 & \multicolumn{2}{|l|}{$\mathrm{C}_{5}$} & & & & & & \\
\hline 4 & 0.99999 & \multicolumn{2}{|l|}{$\mathrm{C}_{6}$} & & & & & & \\
\hline 5 & 0.99999 & \multicolumn{2}{|l|}{$\mathrm{C}_{11}$} & & & & & & \\
\hline 6 & 0.99999 & \multicolumn{2}{|l|}{$\mathrm{C}_{12}$} & & & & & & \\
\hline \multicolumn{10}{|c|}{ 2-center FNO analysis, $\varepsilon=0.90000$} \\
\hline $\mathrm{MO}$ & $\lambda$ & \multicolumn{3}{|c|}{ Atoms } & & & & & \\
\hline 7 & 0.97404 & \multicolumn{3}{|l|}{$\mathrm{C}_{1}-\mathrm{C}_{2}$} & & & & & \\
\hline 8 & 0.97506 & \multicolumn{3}{|l|}{$\mathrm{C}_{1}-\mathrm{H}_{3}$} & & & & & \\
\hline 9 & 0.98090 & \multicolumn{3}{|l|}{$\mathrm{C}_{1}-\mathrm{C}_{5}$} & & & & & \\
\hline 10 & 0.97493 & \multicolumn{3}{|l|}{$\mathrm{C}_{2}-\mathrm{H}_{4}$} & & & & & \\
\hline 11 & 0.98091 & \multicolumn{3}{|l|}{$\mathrm{C}_{2}-\mathrm{C}_{6}$} & & & & & \\
\hline 12 & 0.97006 & \multicolumn{3}{|l|}{$\mathrm{C}_{5}-\mathrm{H}_{7}$} & & & & & \\
\hline 13 & 0.97985 & \multicolumn{3}{|l|}{$\mathrm{C}_{5}-\mathrm{H}_{9}$} & & & & & \\
\hline 14 & 0.97001 & $\mathrm{C}_{6}-\mathrm{H}_{8}$ & & & & & & & \\
\hline 15 & 0.97984 & $\mathrm{C}_{6}-\mathrm{H}_{10}$ & & & & & & & \\
\hline 16 & 0.98250 & $\mathrm{C}_{11}-\mathrm{C}_{12}$ & & & & & & & \\
\hline 17 & 0.97570 & $\mathrm{C}_{11}-\mathrm{H}_{13}$ & & & & & & & \\
\hline 18 & 0.97579 & $\mathrm{C}_{11}-\mathrm{H}_{15}$ & & & & & & & \\
\hline 19 & 0.97555 & $\mathrm{C}_{12}-\mathrm{H}_{14}$ & & & & & & & \\
\hline 20 & 0.97595 & $\mathrm{C}_{12}-\mathrm{H}_{16}$ & & & & & & & \\
\hline 3 -cent & er FNO a & 1alysis, $\varepsilon=$ & 0.90000 & & & & & & \\
\hline $\mathrm{MO}$ & $\lambda$ & Atoms & & & & & & & \\
\hline 21 & 0.93232 & $\mathrm{C}_{1}-\mathrm{C}_{2}-$ & & & & & & & \\
\hline 22 & 0.93228 & $\mathrm{C}_{1}-\mathrm{C}_{2}-$ & & & & & & & \\
\hline 23 & 0.90608 & $\mathrm{C}_{5}-\mathrm{C}_{11}$ & $\mathrm{C}_{12}$ & & & & & & \\
\hline Locali & zation an & I atoms ex & panded 1 & y each & $\mathrm{NO}$ & & & & \\
\hline FNO & $\mathrm{Loc}_{1}$ & $\mathrm{Loc}_{2}$ & $\mathrm{LOC}_{3}$ & $\mathrm{Loc}_{4}$ & $\mathrm{Loc}_{5}$ & $\mathrm{LOc}_{6}$ & $\mathrm{Loc}_{7}$ & $\mathrm{LOc}_{8}$ & $\mathrm{Loc}_{9}$ \\
\hline 1 & $100.0 \%$ & $0.0 \%$ & $0.0 \%$ & $0.0 \%$ & $0.0 \%$ & $0.0 \%$ & $0.0 \%$ & $0.0 \%$ & $0.0 \%$ \\
\hline 2 & $0.0 \%$ & $100.0 \%$ & $0.0 \%$ & $0.0 \%$ & $0.0 \%$ & $0.0 \%$ & $0.0 \%$ & $0.0 \%$ & $0.0 \%$ \\
\hline 3 & $0.0 \%$ & $0.0 \%$ & $0.0 \%$ & $0.0 \%$ & $100.0 \%$ & $0.0 \%$ & $0.0 \%$ & $0.0 \%$ & $0.0 \%$ \\
\hline 4 & $0.0 \%$ & $0.0 \%$ & $0.0 \%$ & $0.0 \%$ & $0.0 \%$ & $100.0 \%$ & $0.0 \%$ & $0.0 \%$ & $0.0 \%$ \\
\hline 5 & $0.0 \%$ & $0.0 \%$ & $0.0 \%$ & $0.0 \%$ & $0.0 \%$ & $0.0 \%$ & $0.0 \%$ & $0.0 \%$ & $0.0 \%$ \\
\hline 6 & $-0.0 \%$ & $0.0 \%$ & $0.0 \%$ & $0.0 \%$ & $0.0 \%$ & $0.0 \%$ & $0.0 \%$ & $0.0 \%$ & $0.0 \%$ \\
\hline 7 & $48.7 \%$ & $48.7 \%$ & $0.5 \%$ & $0.5 \%$ & $0.6 \%$ & $0.6 \%$ & $0.1 \%$ & $0.1 \%$ & $0.1 \%$ \\
\hline 8 & $51.8 \%$ & $0.9 \%$ & $45.7 \%$ & $0.2 \%$ & $1.0 \%$ & $0.1 \%$ & $0.1 \%$ & $0.0 \%$ & $0.2 \%$ \\
\hline 9 & $48.8 \%$ & $0.5 \%$ & $0.4 \%$ & $0.1 \%$ & $49.3 \%$ & $0.1 \%$ & $0.4 \%$ & $0.0 \%$ & $0.4 \%$ \\
\hline 10 & $0.9 \%$ & $51.8 \%$ & $0.2 \%$ & $45.7 \%$ & $0.1 \%$ & $1.0 \%$ & $0.0 \%$ & $0.1 \%$ & $0.0 \%$ \\
\hline 11 & $0.5 \%$ & $48.8 \%$ & $0.1 \%$ & $0.4 \%$ & $0.1 \%$ & $49.3 \%$ & $0.0 \%$ & $0.4 \%$ & $0.0 \%$ \\
\hline 12 & $0.8 \%$ & $0.1 \%$ & $0.1 \%$ & $0.0 \%$ & $52.8 \%$ & $0.1 \%$ & $44.2 \%$ & $0.4 \%$ & $0.6 \%$ \\
\hline 13 & $0.9 \%$ & $0.1 \%$ & $0.2 \%$ & $0.0 \%$ & $53.0 \%$ & $0.0 \%$ & $0.6 \%$ & $0.0 \%$ & $45.0 \%$ \\
\hline 14 & $0.1 \%$ & $0.8 \%$ & $0.0 \%$ & $0.1 \%$ & $0.1 \%$ & $52.8 \%$ & $0.4 \%$ & $44.2 \%$ & $0.0 \%$ \\
\hline 15 & $0.1 \%$ & $0.9 \%$ & $0.0 \%$ & $0.2 \%$ & $0.0 \%$ & $53.0 \%$ & $0.0 \%$ & $0.6 \%$ & $0.0 \%$ \\
\hline 16 & $0.0 \%$ & $0.0 \%$ & $0.0 \%$ & $0.0 \%$ & $0.0 \%$ & $0.0 \%$ & $0.0 \%$ & $0.0 \%$ & $0.0 \%$ \\
\hline 17 & $0.0 \%$ & $0.0 \%$ & $0.0 \%$ & $0.0 \%$ & $0.3 \%$ & $0.0 \%$ & $0.3 \%$ & $0.0 \%$ & $0.0 \%$ \\
\hline 18 & $0.2 \%$ & $0.0 \%$ & $0.0 \%$ & $0.0 \%$ & $0.4 \%$ & $0.0 \%$ & $0.0 \%$ & $0.0 \%$ & $0.0 \%$ \\
\hline 19 & $0.0 \%$ & $0.0 \%$ & $0.0 \%$ & $0.0 \%$ & $0.0 \%$ & $0.3 \%$ & $0.0 \%$ & $0.3 \%$ & $0.0 \%$ \\
\hline 20 & $0.0 \%$ & $0.2 \%$ & $0.0 \%$ & $0.0 \%$ & $0.0 \%$ & $0.4 \%$ & $0.0 \%$ & $0.0 \%$ & $0.0 \%$ \\
\hline 21 & $45.9 \%$ & $9.6 \%$ & $1.6 \%$ & $0.2 \%$ & $37.7 \%$ & $0.4 \%$ & $1.1 \%$ & $0.0 \%$ & $1.3 \%$ \\
\hline 22 & $9.6 \%$ & $45.9 \%$ & $0.2 \%$ & $1.6 \%$ & $0.4 \%$ & $37.7 \%$ & $0.0 \%$ & $1.1 \%$ & $0.0 \%$ \\
\hline 23 & $1.4 \%$ & $0.9 \%$ & $0.1 \%$ & $0.0 \%$ & $0.8 \%$ & $1.2 \%$ & $0.3 \%$ & $0.4 \%$ & $0.0 \%$ \\
\hline
\end{tabular}




\begin{tabular}{crrrrrrrr}
\hline \multicolumn{2}{c}{ Localization and atoms expanded by each FNO } \\
\hline FNO & Loc $_{10}$ & Loc $_{11}$ & Loc $_{12}$ & Loc $_{13}$ & Loc $_{14}$ & Loc $_{15}$ & Loc $_{16}$ & $n_{\text {eff }}$ \\
\hline 1 & $0.0 \%$ & $0.0 \%$ & $0.0 \%$ & $0.0 \%$ & $0.0 \%$ & $0.0 \%$ & $0.0 \%$ & 1.00002 \\
2 & $0.0 \%$ & $0.0 \%$ & $0.0 \%$ & $0.0 \%$ & $0.0 \%$ & $0.0 \%$ & $0.0 \%$ & 1.00002 \\
3 & $0.0 \%$ & $0.0 \%$ & $0.0 \%$ & $0.0 \%$ & $0.0 \%$ & $0.0 \%$ & $0.0 \%$ & 1.00002 \\
4 & $0.0 \%$ & $0.0 \%$ & $0.0 \%$ & $0.0 \%$ & $0.0 \%$ & $0.0 \%$ & $0.0 \%$ & 1.00002 \\
5 & $0.0 \%$ & $100.0 \%$ & $0.0 \%$ & $0.0 \%$ & $0.0 \%$ & $0.0 \%$ & $0.0 \%$ & 1.00002 \\
6 & $0.0 \%$ & $0.0 \% 1$ & $00.0 \%$ & $0.0 \%$ & $0.0 \%$ & $0.0 \%$ & $0.0 \%$ & 1.00002 \\
7 & $0.1 \%$ & $0.0 \%$ & $0.0 \%$ & $0.0 \%$ & $0.0 \%$ & $0.0 \%$ & $0.0 \%$ & 2.10745 \\
8 & $0.0 \%$ & $0.0 \%$ & $0.0 \%$ & $0.0 \%$ & $0.0 \%$ & $0.0 \%$ & $0.0 \%$ & 2.09447 \\
9 & $0.0 \%$ & $0.1 \%$ & $0.0 \%$ & $0.0 \%$ & $0.0 \%$ & $0.0 \%$ & $0.0 \%$ & 2.07828 \\
10 & $0.2 \%$ & $0.0 \%$ & $0.0 \%$ & $0.0 \%$ & $0.0 \%$ & $0.0 \%$ & $0.0 \%$ & 2.09503 \\
11 & $0.4 \%$ & $0.0 \%$ & $0.1 \%$ & $0.0 \%$ & $0.0 \%$ & $0.0 \%$ & $0.0 \%$ & 2.07824 \\
12 & $0.0 \%$ & $0.4 \%$ & $0.1 \%$ & $0.2 \%$ & $0.0 \%$ & $0.0 \%$ & $0.0 \%$ & 2.10813 \\
13 & $0.0 \%$ & $0.1 \%$ & $0.0 \%$ & $0.0 \%$ & $0.0 \%$ & $0.0 \%$ & $0.0 \%$ & 2.06873 \\
14 & $0.6 \%$ & $0.1 \%$ & $0.4 \%$ & $0.0 \%$ & $0.2 \%$ & $0.0 \%$ & $0.0 \%$ & 2.10834 \\
15 & $45.0 \%$ & $0.0 \%$ & $0.1 \%$ & $0.0 \%$ & $0.0 \%$ & $0.0 \%$ & $0.0 \%$ & 2.06879 \\
16 & $0.0 \%$ & $49.1 \%$ & $49.1 \%$ & $0.4 \%$ & $0.4 \%$ & $0.4 \%$ & $0.4 \%$ & 2.07160 \\
17 & $0.0 \%$ & $52.3 \%$ & $0.9 \%$ & $45.3 \%$ & $0.1 \%$ & $0.6 \%$ & $0.1 \%$ & 2.08957 \\
18 & $0.0 \%$ & $52.8 \%$ & $0.9 \%$ & $0.6 \%$ & $0.1 \%$ & $44.8 \%$ & $0.1 \%$ & 2.08592 \\
19 & $0.0 \%$ & $0.9 \%$ & $52.3 \%$ & $0.1 \%$ & $45.3 \%$ & $0.1 \%$ & $0.6 \%$ & 2.09022 \\
20 & $0.0 \%$ & $0.9 \%$ & $52.8 \%$ & $0.1 \%$ & $0.6 \%$ & $0.1 \%$ & $44.8 \%$ & 2.08522 \\
21 & $0.0 \%$ & $1.1 \%$ & $0.5 \%$ & $0.1 \%$ & $0.0 \%$ & $0.4 \%$ & $0.0 \%$ & 2.75375 \\
22 & $1.3 \%$ & $0.5 \%$ & $1.1 \%$ & $0.0 \%$ & $0.1 \%$ & $0.0 \%$ & $0.4 \%$ & 2.75447 \\
23 & $0.0 \%$ & $44.1 \%$ & $45.2 \%$ & $1.5 \%$ & $1.5 \%$ & $1.3 \%$ & $1.4 \%$ & 2.50159 \\
\hline \hline
\end{tabular}

\section{S1.4.8 IRC Step 14}

\begin{tabular}{ccl}
\hline \hline \multicolumn{3}{c}{ 1-center FNO analysis, $\varepsilon=0.90000$} \\
\hline MO & \multicolumn{1}{c}{$\lambda$} & Atoms \\
\hline 1 & 0.99999 & $\mathrm{C}_{1}$ \\
2 & 0.99999 & $\mathrm{C}_{2}$ \\
3 & 0.99999 & $\mathrm{C}_{5}$ \\
4 & 0.99999 & $\mathrm{C}_{6}$ \\
5 & 0.99999 & $\mathrm{C}_{11}$ \\
6 & 0.99999 & $\mathrm{C}_{12}$ \\
\hline 2 -center FNO & analysis, $\varepsilon=0.90000$ \\
\hline $\mathrm{MO}$ & \multicolumn{1}{c}{$\lambda$} & Atoms \\
\hline 7 & 0.97392 & $\mathrm{C}_{1}-\mathrm{C}_{2}$ \\
8 & 0.97512 & $\mathrm{C}_{1}-\mathrm{H}_{3}$ \\
9 & 0.98043 & $\mathrm{C}_{1}-\mathrm{C}_{5}$ \\
10 & 0.97498 & $\mathrm{C}_{2}-\mathrm{H}_{4}$ \\
11 & 0.98048 & $\mathrm{C}_{2}-\mathrm{C}_{6}$ \\
12 & 0.96850 & $\mathrm{C}_{5}-\mathrm{H}_{7}$ \\
13 & 0.97933 & $\mathrm{C}_{5}-\mathrm{H}_{9}$ \\
14 & 0.96840 & $\mathrm{C}_{6}-\mathrm{H}_{8}$ \\
15 & 0.97941 & $\mathrm{C}_{6}-\mathrm{H}_{10}$ \\
16 & 0.98186 & $\mathrm{C}_{11}-\mathrm{C}_{12}$ \\
17 & 0.97456 & $\mathrm{C}_{11}-\mathrm{H}_{13}$ \\
18 & 0.97489 & $\mathrm{C}_{11}-\mathrm{H}_{15}$ \\
19 & 0.97434 & $\mathrm{C}_{12}-\mathrm{H}_{14}$ \\
20 & 0.97506 & $\mathrm{C}_{12}-\mathrm{H}_{16}$ \\
\hline 3 -center $\mathrm{FNO}$ analysis, $\varepsilon=0.90000$ \\
\hline MO & $\lambda$ & Atoms $_{2}$ \\
\hline 21 & 0.92916 & $\mathrm{C}_{1}-\mathrm{C}_{2}-\mathrm{C}_{5}$ \\
22 & 0.92905 & $\mathrm{C}_{1}-\mathrm{C}_{2}-\mathrm{C}_{6}$ \\
23 & 0.90976 & $\mathrm{C}_{5}-\mathrm{C}_{11}-\mathrm{C}_{12}$ \\
\hline \hline & &
\end{tabular}




\begin{tabular}{crrrrrrrrr}
\hline \multicolumn{2}{c}{ Localization and atoms expanded by each FNO } \\
\hline FNO & Loc $_{1}$ & $\mathrm{Loc}_{2}$ & $\mathrm{LOc}_{3}$ & $\mathrm{Loc}_{4}$ & $\mathrm{Loc}_{5}$ & $\mathrm{Loc}_{6}$ & $\mathrm{Loc}_{7}$ & $\mathrm{Loc}_{8}$ & Loc $_{9}$ \\
\hline 1 & $100.0 \%$ & $0.0 \%$ & $0.0 \%$ & $0.0 \%$ & $0.0 \%$ & $0.0 \%$ & $0.0 \%$ & $0.0 \%$ & $0.0 \%$ \\
2 & $0.0 \%$ & $100.0 \%$ & $0.0 \%$ & $0.0 \%$ & $0.0 \%$ & $0.0 \%$ & $0.0 \%$ & $0.0 \%$ & $0.0 \%$ \\
3 & $0.0 \%$ & $0.0 \%$ & $0.0 \%$ & $0.0 \%$ & $100.0 \%$ & $0.0 \%$ & $0.0 \%$ & $0.0 \%$ & $0.0 \%$ \\
4 & $0.0 \%$ & $0.0 \%$ & $0.0 \%$ & $0.0 \%$ & $0.0 \%$ & $100.0 \%$ & $0.0 \%$ & $0.0 \%$ & $0.0 \%$ \\
5 & $-0.0 \%$ & $0.0 \%$ & $0.0 \%$ & $0.0 \%$ & $0.0 \%$ & $0.0 \%$ & $0.0 \%$ & $0.0 \%$ & $0.0 \%$ \\
6 & $-0.0 \%$ & $0.0 \%$ & $0.0 \%$ & $0.0 \%$ & $0.0 \%$ & $0.0 \%$ & $0.0 \%$ & $0.0 \%$ & $0.0 \%$ \\
7 & $48.7 \%$ & $48.7 \%$ & $0.5 \%$ & $0.5 \%$ & $0.6 \%$ & $0.6 \%$ & $0.1 \%$ & $0.1 \%$ & $0.1 \%$ \\
8 & $51.8 \%$ & $0.9 \%$ & $45.7 \%$ & $0.2 \%$ & $1.0 \%$ & $0.1 \%$ & $0.1 \%$ & $0.0 \%$ & $0.2 \%$ \\
9 & $48.6 \%$ & $0.5 \%$ & $0.4 \%$ & $0.1 \%$ & $49.5 \%$ & $0.1 \%$ & $0.4 \%$ & $0.0 \%$ & $0.4 \%$ \\
10 & $0.9 \%$ & $51.8 \%$ & $0.2 \%$ & $45.7 \%$ & $0.1 \%$ & $1.0 \%$ & $0.0 \%$ & $0.1 \%$ & $0.0 \%$ \\
11 & $0.5 \%$ & $48.6 \%$ & $0.1 \%$ & $0.4 \%$ & $0.1 \%$ & $49.5 \%$ & $0.0 \%$ & $0.4 \%$ & $0.0 \%$ \\
12 & $0.8 \%$ & $0.1 \%$ & $0.1 \%$ & $0.0 \%$ & $52.7 \%$ & $0.1 \%$ & $44.1 \%$ & $0.4 \%$ & $0.6 \%$ \\
13 & $0.9 \%$ & $0.1 \%$ & $0.2 \%$ & $0.0 \%$ & $52.9 \%$ & $0.0 \%$ & $0.6 \%$ & $0.0 \%$ & $45.0 \%$ \\
14 & $0.2 \%$ & $0.8 \%$ & $0.0 \%$ & $0.1 \%$ & $0.1 \%$ & $52.7 \%$ & $0.4 \%$ & $44.1 \%$ & $0.0 \%$ \\
15 & $0.1 \%$ & $0.9 \%$ & $0.0 \%$ & $0.2 \%$ & $0.0 \%$ & $52.9 \%$ & $0.0 \%$ & $0.6 \%$ & $0.0 \%$ \\
16 & $0.0 \%$ & $0.0 \%$ & $0.0 \%$ & $0.0 \%$ & $0.1 \%$ & $0.1 \%$ & $0.0 \%$ & $0.0 \%$ & $0.0 \%$ \\
17 & $0.0 \%$ & $0.0 \%$ & $0.0 \%$ & $0.0 \%$ & $0.4 \%$ & $0.0 \%$ & $0.3 \%$ & $0.0 \%$ & $0.0 \%$ \\
18 & $0.2 \%$ & $0.0 \%$ & $0.0 \%$ & $0.0 \%$ & $0.4 \%$ & $0.0 \%$ & $0.0 \%$ & $0.0 \%$ & $0.0 \%$ \\
19 & $0.0 \%$ & $0.0 \%$ & $0.0 \%$ & $0.0 \%$ & $0.0 \%$ & $0.4 \%$ & $0.0 \%$ & $0.3 \%$ & $0.0 \%$ \\
20 & $0.0 \%$ & $0.2 \%$ & $0.0 \%$ & $0.0 \%$ & $0.0 \%$ & $0.4 \%$ & $0.0 \%$ & $0.0 \%$ & $0.0 \%$ \\
21 & $45.9 \%$ & $10.6 \%$ & $1.6 \%$ & $0.3 \%$ & $36.4 \%$ & $0.5 \%$ & $1.0 \%$ & $0.0 \%$ & $1.2 \%$ \\
22 & $10.6 \%$ & $45.9 \%$ & $0.3 \%$ & $1.6 \%$ & $0.5 \%$ & $36.4 \%$ & $0.0 \%$ & $1.0 \%$ & $0.0 \%$ \\
23 & $0.5 \%$ & $0.7 \%$ & $0.0 \%$ & $0.0 \%$ & $3.8 \%$ & $1.1 \%$ & $0.6 \%$ & $0.3 \%$ & $0.1 \%$ \\
\hline \hline \hline
\end{tabular}

\begin{tabular}{crrrrrrrr}
\hline \hline \multicolumn{7}{c}{ Localization and atoms expanded by each FNO } \\
\hline FNO & Loc $_{10}$ & Loc $_{11}$ & Loc $_{12}$ & Loc $_{13}$ & Loc $_{14}$ & Loc $_{15}$ & Loc $_{16}$ & $n_{\text {eff }}$ \\
\hline 1 & $0.0 \%$ & $0.0 \%$ & $0.0 \%$ & $0.0 \%$ & $0.0 \%$ & $0.0 \%$ & $0.0 \%$ & 1.00002 \\
2 & $0.0 \%$ & $0.0 \%$ & $0.0 \%$ & $0.0 \%$ & $0.0 \%$ & $0.0 \%$ & $0.0 \%$ & 1.00002 \\
3 & $0.0 \%$ & $0.0 \%$ & $0.0 \%$ & $0.0 \%$ & $0.0 \%$ & $0.0 \%$ & $0.0 \%$ & 1.00002 \\
4 & $0.0 \%$ & $0.0 \%$ & $0.0 \%$ & $0.0 \%$ & $0.0 \%$ & $0.0 \%$ & $0.0 \%$ & 1.00002 \\
5 & $0.0 \%$ & $100.0 \%$ & $0.0 \%$ & $0.0 \%$ & $0.0 \%$ & $0.0 \%$ & $0.0 \%$ & 1.00002 \\
6 & $0.0 \%$ & $0.0 \%$ & $100.0 \%$ & $0.0 \%$ & $0.0 \%$ & $0.0 \%$ & $0.0 \%$ & 1.00002 \\
7 & $0.1 \%$ & $0.0 \%$ & $0.0 \%$ & $0.0 \%$ & $0.0 \%$ & $0.0 \%$ & $0.0 \%$ & 2.10796 \\
8 & $0.0 \%$ & $0.0 \%$ & $0.0 \%$ & $0.0 \%$ & $0.0 \%$ & $0.0 \%$ & $0.0 \%$ & 2.09417 \\
9 & $0.0 \%$ & $0.1 \%$ & $0.0 \%$ & $0.0 \%$ & $0.0 \%$ & $0.0 \%$ & $0.0 \%$ & 2.08016 \\
10 & $0.2 \%$ & $0.0 \%$ & $0.0 \%$ & $0.0 \%$ & $0.0 \%$ & $0.0 \%$ & $0.0 \%$ & 2.09479 \\
11 & $0.4 \%$ & $0.0 \%$ & $0.1 \%$ & $0.0 \%$ & $0.0 \%$ & $0.0 \%$ & $0.0 \%$ & 2.07997 \\
12 & $0.0 \%$ & $0.5 \%$ & $0.1 \%$ & $0.2 \%$ & $0.0 \%$ & $0.0 \%$ & $0.0 \%$ & 2.11492 \\
13 & $0.0 \%$ & $0.1 \%$ & $0.0 \%$ & $0.0 \%$ & $0.0 \%$ & $0.1 \%$ & $0.0 \%$ & 2.07125 \\
14 & $0.6 \%$ & $0.1 \%$ & $0.5 \%$ & $0.0 \%$ & $0.2 \%$ & $0.0 \%$ & $0.0 \%$ & 2.11535 \\
15 & $45.0 \%$ & $0.0 \%$ & $0.1 \%$ & $0.0 \%$ & $0.0 \%$ & $0.0 \%$ & $0.1 \%$ & 2.07092 \\
16 & $0.0 \%$ & $49.1 \%$ & $49.1 \%$ & $0.4 \%$ & $0.4 \%$ & $0.4 \%$ & $0.4 \%$ & 2.07431 \\
17 & $0.0 \%$ & $52.2 \%$ & $0.9 \%$ & $45.3 \%$ & $0.1 \%$ & $0.6 \%$ & $0.1 \%$ & 2.09448 \\
18 & $0.0 \%$ & $52.7 \%$ & $0.9 \%$ & $0.6 \%$ & $0.1 \%$ & $44.8 \%$ & $0.1 \%$ & 2.08983 \\
19 & $0.0 \%$ & $0.9 \%$ & $52.2 \%$ & $0.1 \%$ & $45.2 \%$ & $0.1 \%$ & $0.6 \%$ & 2.09546 \\
20 & $0.0 \%$ & $0.9 \%$ & $52.7 \%$ & $0.1 \%$ & $0.6 \%$ & $0.1 \%$ & $44.8 \%$ & 2.08905 \\
21 & $0.0 \%$ & $1.3 \%$ & $0.7 \%$ & $0.1 \%$ & $0.0 \%$ & $0.4 \%$ & $0.0 \%$ & 2.81540 \\
22 & $1.2 \%$ & $0.7 \%$ & $1.3 \%$ & $0.0 \%$ & $0.1 \%$ & $0.0 \%$ & $0.4 \%$ & 2.81671 \\
23 & $0.0 \%$ & $45.3 \%$ & $41.9 \%$ & $1.6 \%$ & $1.4 \%$ & $1.5 \%$ & $1.3 \%$ & 2.61255 \\
\hline \hline
\end{tabular}




\section{S1.4.9 IRC Step 16}

\begin{tabular}{|c|c|c|c|c|c|c|c|c|c|}
\hline \multicolumn{4}{|c|}{ 1-center FNO analysis, $\varepsilon=0.90000$} & & & & & & \\
\hline MO & $\lambda$ & \multicolumn{2}{|l|}{ Atoms } & & & & & & \\
\hline 1 & 0.99999 & \multicolumn{2}{|l|}{$\mathrm{C}_{1}$} & & & & & & \\
\hline 2 & 0.99999 & \multicolumn{2}{|l|}{$\mathrm{C}_{2}$} & & & & & & \\
\hline 3 & 0.99999 & \multicolumn{2}{|l|}{$\mathrm{C}_{5}$} & & & & & & \\
\hline 4 & 0.99999 & \multicolumn{2}{|l|}{$\mathrm{C}_{6}$} & & & & & & \\
\hline 5 & 0.99999 & \multicolumn{2}{|l|}{$\mathrm{C}_{11}$} & & & & & & \\
\hline 6 & 0.99999 & \multicolumn{2}{|l|}{$\mathrm{C}_{12}$} & & & & & & \\
\hline \multicolumn{10}{|c|}{ 2-center FNO analysis, $\varepsilon=0.90000$} \\
\hline $\mathrm{MO}$ & $\lambda$ & \multicolumn{3}{|c|}{ Atoms } & & & & & \\
\hline 7 & 0.97398 & \multicolumn{3}{|l|}{$\mathrm{C}_{1}-\mathrm{C}_{2}$} & & & & & \\
\hline 8 & 0.97516 & \multicolumn{3}{|l|}{$\mathrm{C}_{1}-\mathrm{H}_{3}$} & & & & & \\
\hline 9 & 0.97977 & \multicolumn{3}{|l|}{$\mathrm{C}_{1}-\mathrm{C}_{5}$} & & & & & \\
\hline 10 & 0.97504 & \multicolumn{3}{|l|}{$\mathrm{C}_{2}-\mathrm{H}_{4}$} & & & & & \\
\hline 11 & 0.97979 & \multicolumn{3}{|l|}{$\mathrm{C}_{2}-\mathrm{C}_{6}$} & & & & & \\
\hline 12 & 0.96705 & \multicolumn{3}{|l|}{$\mathrm{C}_{5}-\mathrm{H}_{7}$} & & & & & \\
\hline 13 & 0.97880 & \multicolumn{3}{|l|}{$\mathrm{C}_{5}-\mathrm{H}_{9}$} & & & & & \\
\hline 14 & 0.96685 & $\mathrm{C}_{6}-\mathrm{H}_{8}$ & & & & & & & \\
\hline 15 & 0.97870 & $\mathrm{C}_{6}-\mathrm{H}_{10}$ & & & & & & & \\
\hline 16 & 0.98118 & $\mathrm{C}_{11}-\mathrm{C}_{12}$ & & & & & & & \\
\hline 17 & 0.97324 & $\mathrm{C}_{11}-\mathrm{H}_{13}$ & & & & & & & \\
\hline 18 & 0.97437 & $\mathrm{C}_{11}-\mathrm{H}_{15}$ & & & & & & & \\
\hline 19 & 0.97328 & $\mathrm{C}_{12}-\mathrm{H}_{14}$ & & & & & & & \\
\hline 20 & 0.97421 & $\mathrm{C}_{12}-\mathrm{H}_{16}$ & & & & & & & \\
\hline 3 -cent & er FNO a & 1alysis, $\varepsilon=$ & 0.90000 & & & & & & \\
\hline $\mathrm{MO}$ & $\lambda$ & Atoms & & & & & & & \\
\hline 21 & 0.93232 & $\mathrm{C}_{1}-\mathrm{C}_{2}-$ & & & & & & & \\
\hline 22 & 0.93228 & $\mathrm{C}_{1}-\mathrm{C}_{2}-$ & & & & & & & \\
\hline 23 & 0.90608 & $\mathrm{C}_{5}-\mathrm{C}_{11}$ & $\mathrm{C}_{12}$ & & & & & & \\
\hline Locali & zation an & I atoms ex & panded 1 & y each & $\mathrm{NO}$ & & & & \\
\hline FNO & $\mathrm{Loc}_{1}$ & $\mathrm{Loc}_{2}$ & $\mathrm{LOC}_{3}$ & $\mathrm{Loc}_{4}$ & $\mathrm{Loc}_{5}$ & $\mathrm{LOc}_{6}$ & $\mathrm{Loc}_{7}$ & $\mathrm{LOc}_{8}$ & $\mathrm{Loc}_{9}$ \\
\hline 1 & $100.0 \%$ & $0.0 \%$ & $0.0 \%$ & $0.0 \%$ & $0.0 \%$ & $0.0 \%$ & $0.0 \%$ & $0.0 \%$ & $0.0 \%$ \\
\hline 2 & $0.0 \%$ & $100.0 \%$ & $0.0 \%$ & $0.0 \%$ & $0.0 \%$ & $0.0 \%$ & $0.0 \%$ & $0.0 \%$ & $0.0 \%$ \\
\hline 3 & $0.0 \%$ & $0.0 \%$ & $0.0 \%$ & $0.0 \%$ & $100.0 \%$ & $0.0 \%$ & $0.0 \%$ & $0.0 \%$ & $0.0 \%$ \\
\hline 4 & $0.0 \%$ & $0.0 \%$ & $0.0 \%$ & $0.0 \%$ & $0.0 \%$ & $100.0 \%$ & $0.0 \%$ & $0.0 \%$ & $0.0 \%$ \\
\hline 5 & $-0.0 \%$ & $0.0 \%$ & $0.0 \%$ & $0.0 \%$ & $0.0 \%$ & $0.0 \%$ & $0.0 \%$ & $0.0 \%$ & $0.0 \%$ \\
\hline 6 & $-0.0 \%$ & $0.0 \%$ & $0.0 \%$ & $0.0 \%$ & $0.0 \%$ & $0.0 \%$ & $0.0 \%$ & $0.0 \%$ & $0.0 \%$ \\
\hline 7 & $48.7 \%$ & $48.7 \%$ & $0.5 \%$ & $0.5 \%$ & $0.6 \%$ & $0.6 \%$ & $0.1 \%$ & $0.1 \%$ & $0.1 \%$ \\
\hline 8 & $51.8 \%$ & $0.9 \%$ & $45.7 \%$ & $0.2 \%$ & $1.0 \%$ & $0.1 \%$ & $0.1 \%$ & $0.0 \%$ & $0.2 \%$ \\
\hline 9 & $48.4 \%$ & $0.5 \%$ & $0.4 \%$ & $0.1 \%$ & $49.6 \%$ & $0.1 \%$ & $0.4 \%$ & $0.0 \%$ & $0.4 \%$ \\
\hline 10 & $0.9 \%$ & $51.8 \%$ & $0.2 \%$ & $45.7 \%$ & $0.1 \%$ & $1.0 \%$ & $0.0 \%$ & $0.1 \%$ & $0.0 \%$ \\
\hline 11 & $0.5 \%$ & $48.4 \%$ & $0.1 \%$ & $0.4 \%$ & $0.1 \%$ & $49.6 \%$ & $0.0 \%$ & $0.4 \%$ & $0.0 \%$ \\
\hline 12 & $0.8 \%$ & $0.1 \%$ & $0.1 \%$ & $0.0 \%$ & $52.6 \%$ & $0.1 \%$ & $44.1 \%$ & $0.4 \%$ & $0.6 \%$ \\
\hline 13 & $0.9 \%$ & $0.1 \%$ & $0.2 \%$ & $0.0 \%$ & $52.8 \%$ & $0.0 \%$ & $0.6 \%$ & $0.0 \%$ & $45.0 \%$ \\
\hline 14 & $0.2 \%$ & $0.8 \%$ & $0.0 \%$ & $0.1 \%$ & $0.1 \%$ & $52.6 \%$ & $0.4 \%$ & $44.1 \%$ & $0.0 \%$ \\
\hline 15 & $0.1 \%$ & $0.9 \%$ & $0.0 \%$ & $0.2 \%$ & $0.0 \%$ & $52.8 \%$ & $0.0 \%$ & $0.6 \%$ & $0.0 \%$ \\
\hline 16 & $0.0 \%$ & $0.0 \%$ & $0.0 \%$ & $0.0 \%$ & $0.1 \%$ & $0.1 \%$ & $0.0 \%$ & $0.0 \%$ & $0.0 \%$ \\
\hline 17 & $0.1 \%$ & $0.0 \%$ & $0.0 \%$ & $0.0 \%$ & $0.4 \%$ & $0.0 \%$ & $0.3 \%$ & $0.0 \%$ & $0.0 \%$ \\
\hline 18 & $0.2 \%$ & $0.0 \%$ & $0.0 \%$ & $0.0 \%$ & $0.5 \%$ & $0.0 \%$ & $0.0 \%$ & $0.0 \%$ & $0.0 \%$ \\
\hline 19 & $0.0 \%$ & $0.1 \%$ & $0.0 \%$ & $0.0 \%$ & $0.0 \%$ & $0.4 \%$ & $0.0 \%$ & $0.3 \%$ & $0.0 \%$ \\
\hline 20 & $0.0 \%$ & $0.2 \%$ & $0.0 \%$ & $0.0 \%$ & $0.0 \%$ & $0.5 \%$ & $0.0 \%$ & $0.0 \%$ & $0.0 \%$ \\
\hline 21 & $45.9 \%$ & $12.4 \%$ & $1.6 \%$ & $0.3 \%$ & $34.2 \%$ & $0.6 \%$ & $0.9 \%$ & $0.0 \%$ & $1.1 \%$ \\
\hline 22 & $12.5 \%$ & $45.9 \%$ & $0.3 \%$ & $1.6 \%$ & $0.6 \%$ & $34.2 \%$ & $0.0 \%$ & $0.9 \%$ & $0.0 \%$ \\
\hline 23 & $0.6 \%$ & $0.9 \%$ & $0.0 \%$ & $0.1 \%$ & $6.1 \%$ & $1.4 \%$ & $0.7 \%$ & $0.3 \%$ & $0.2 \%$ \\
\hline
\end{tabular}




\begin{tabular}{crrrrrrrr}
\hline \multicolumn{2}{l}{ localization and atoms expanded by each FNO } \\
\hline FNO & Loc $_{10}$ & Loc $_{11}$ & Loc $_{12}$ & Loc $_{13}$ & Loc $_{14}$ & Loc $_{15}$ & Loc $_{16}$ & $n_{\text {eff }}$ \\
\hline 1 & $0.0 \%$ & $0.0 \%$ & $0.0 \%$ & $0.0 \%$ & $0.0 \%$ & $0.0 \%$ & $0.0 \%$ & 1.00002 \\
2 & $0.0 \%$ & $0.0 \%$ & $0.0 \%$ & $0.0 \%$ & $0.0 \%$ & $0.0 \%$ & $0.0 \%$ & 1.00002 \\
3 & $0.0 \%$ & $0.0 \%$ & $0.0 \%$ & $0.0 \%$ & $0.0 \%$ & $0.0 \%$ & $0.0 \%$ & 1.00002 \\
4 & $0.0 \%$ & $0.0 \%$ & $0.0 \%$ & $0.0 \%$ & $0.0 \%$ & $0.0 \%$ & $0.0 \%$ & 1.00002 \\
5 & $0.0 \%$ & $100.0 \%$ & $0.0 \%$ & $0.0 \%$ & $0.0 \%$ & $0.0 \%$ & $0.0 \%$ & 1.00002 \\
6 & $0.0 \%$ & $0.0 \%$ & $100.0 \%$ & $0.0 \%$ & $0.0 \%$ & $0.0 \%$ & $0.0 \%$ & 1.00002 \\
7 & $0.1 \%$ & $0.0 \%$ & $0.0 \%$ & $0.0 \%$ & $0.0 \%$ & $0.0 \%$ & $0.0 \%$ & 2.10769 \\
8 & $0.0 \%$ & $0.0 \%$ & $0.0 \%$ & $0.0 \%$ & $0.0 \%$ & $0.0 \%$ & $0.0 \%$ & 2.09399 \\
9 & $0.0 \%$ & $0.1 \%$ & $0.0 \%$ & $0.0 \%$ & $0.0 \%$ & $0.0 \%$ & $0.0 \%$ & 2.08283 \\
10 & $0.2 \%$ & $0.0 \%$ & $0.0 \%$ & $0.0 \%$ & $0.0 \%$ & $0.0 \%$ & $0.0 \%$ & 2.09453 \\
11 & $0.4 \%$ & $0.0 \%$ & $0.1 \%$ & $0.0 \%$ & $0.0 \%$ & $0.0 \%$ & $0.0 \%$ & 2.08271 \\
12 & $0.0 \%$ & $0.6 \%$ & $0.1 \%$ & $0.2 \%$ & $0.0 \%$ & $0.0 \%$ & $0.0 \%$ & 2.12140 \\
13 & $0.0 \%$ & $0.2 \%$ & $0.0 \%$ & $0.1 \%$ & $0.0 \%$ & $0.1 \%$ & $0.0 \%$ & 2.07392 \\
14 & $0.6 \%$ & $0.1 \%$ & $0.6 \%$ & $0.0 \%$ & $0.2 \%$ & $0.0 \%$ & $0.0 \%$ & 2.12231 \\
15 & $45.0 \%$ & $0.0 \%$ & $0.2 \%$ & $0.0 \%$ & $0.1 \%$ & $0.0 \%$ & $0.1 \%$ & 2.07435 \\
16 & $0.0 \%$ & $49.1 \%$ & $49.1 \%$ & $0.4 \%$ & $0.4 \%$ & $0.4 \%$ & $0.4 \%$ & 2.07717 \\
17 & $0.0 \%$ & $52.2 \%$ & $0.9 \%$ & $45.2 \%$ & $0.1 \%$ & $0.6 \%$ & $0.1 \%$ & 2.10000 \\
18 & $0.0 \%$ & $52.7 \%$ & $0.9 \%$ & $0.6 \%$ & $0.1 \%$ & $44.7 \%$ & $0.1 \%$ & 2.09194 \\
19 & $0.0 \%$ & $0.9 \%$ & $52.2 \%$ & $0.1 \%$ & $45.2 \%$ & $0.1 \%$ & $0.6 \%$ & 2.09980 \\
20 & $0.0 \%$ & $0.9 \%$ & $52.7 \%$ & $0.1 \%$ & $0.6 \%$ & $0.1 \%$ & $44.7 \%$ & 2.09263 \\
21 & $0.0 \%$ & $1.5 \%$ & $0.8 \%$ & $0.1 \%$ & $0.1 \%$ & $0.4 \%$ & $0.1 \%$ & 2.91058 \\
22 & $1.1 \%$ & $0.8 \%$ & $1.5 \%$ & $0.1 \%$ & $0.1 \%$ & $0.1 \%$ & $0.4 \%$ & 2.91173 \\
23 & $0.0 \%$ & $44.9 \%$ & $39.3 \%$ & $1.6 \%$ & $1.3 \%$ & $1.5 \%$ & $1.2 \%$ & 2.77181 \\
\hline \hline
\end{tabular}

\section{S1.4.10 IRC Step 18}

\begin{tabular}{|c|c|c|}
\hline \multicolumn{3}{|c|}{1 -center FNO analysis, $\varepsilon=0.90000$} \\
\hline MO & $\lambda$ & Atoms \\
\hline 1 & 0.99999 & $\mathrm{C}_{1}$ \\
\hline 2 & 0.99999 & $\mathrm{C}_{2}$ \\
\hline 3 & 0.99999 & $\mathrm{C}_{5}$ \\
\hline 4 & 0.99999 & $\mathrm{C}_{6}$ \\
\hline 5 & 0.99999 & $\mathrm{C}_{11}$ \\
\hline 6 & 0.99999 & $\mathrm{C}_{12}$ \\
\hline \multicolumn{3}{|c|}{ 2-center FNO analysis, $\varepsilon=0.90000$} \\
\hline $\mathrm{MO}$ & $\lambda$ & Atoms \\
\hline 7 & 0.97432 & $\mathrm{C}_{1}-\mathrm{C}_{2}$ \\
\hline 8 & 0.97524 & $\mathrm{C}_{1}-\mathrm{H}_{3}$ \\
\hline 9 & 0.97875 & $\mathrm{C}_{1}-\mathrm{C}_{5}$ \\
\hline 10 & 0.97509 & $\mathrm{C}_{2}-\mathrm{H}_{4}$ \\
\hline 11 & 0.97881 & $\mathrm{C}_{2}-\mathrm{C}_{6}$ \\
\hline 12 & 0.96561 & $\mathrm{C}_{5}-\mathrm{H}_{7}$ \\
\hline 13 & 0.97808 & $\mathrm{C}_{5}-\mathrm{H}_{9}$ \\
\hline 14 & 0.96565 & $\mathrm{C}_{6}-\mathrm{H}_{8}$ \\
\hline 15 & 0.97814 & $\mathrm{C}_{6}-\mathrm{H}_{10}$ \\
\hline 16 & 0.98003 & $\mathrm{C}_{11}-\mathrm{C}_{12}$ \\
\hline 17 & 0.97247 & $\mathrm{C}_{11}-\mathrm{H}_{13}$ \\
\hline 18 & 0.97371 & $\mathrm{C}_{11}-\mathrm{H}_{15}$ \\
\hline 19 & 0.97251 & $\mathrm{C}_{12}-\mathrm{H}_{14}$ \\
\hline 20 & 0.97355 & $\mathrm{C}_{12}-\mathrm{H}_{16}$ \\
\hline \multicolumn{3}{|c|}{3 -center FNO analysis, $\varepsilon=0.90000$} \\
\hline $\mathrm{MO}$ & $\lambda$ & Atoms \\
\hline 21 & 0.91938 & $\mathrm{C}_{1}-\mathrm{C}_{2}-\mathrm{C}_{5}$ \\
\hline 22 & 0.91939 & $\mathrm{C}_{1}-\mathrm{C}_{2}-\mathrm{C}_{6}$ \\
\hline \multicolumn{3}{|c|}{ 4-center FNO analysis, $\varepsilon=0.90000$} \\
\hline MO & $\lambda$ & Atoms \\
\hline 23 & 0.91286 & $\mathrm{C}_{5}-\mathrm{C}_{6}-\mathrm{C}_{11}-\mathrm{C}_{12}$ \\
\hline
\end{tabular}




\begin{tabular}{crrrrrrrrr}
\hline \multicolumn{2}{c}{ Localization and atoms expanded by each FNO } \\
\hline FNO & Loc $_{1}$ & $\mathrm{Loc}_{2}$ & $\mathrm{Loc}_{3}$ & $\mathrm{Loc}_{4}$ & $\mathrm{Loc}_{5}$ & $\mathrm{Loc}_{6}$ & $\mathrm{Loc}_{7}$ & $\mathrm{Loc}_{8}$ & Loc $_{9}$ \\
\hline 1 & $100.0 \%$ & $0.0 \%$ & $0.0 \%$ & $0.0 \%$ & $0.0 \%$ & $0.0 \%$ & $0.0 \%$ & $0.0 \%$ & $0.0 \%$ \\
2 & $0.0 \%$ & $100.0 \%$ & $0.0 \%$ & $0.0 \%$ & $0.0 \%$ & $0.0 \%$ & $0.0 \%$ & $0.0 \%$ & $0.0 \%$ \\
3 & $0.0 \%$ & $0.0 \%$ & $0.0 \%$ & $0.0 \%$ & $100.0 \%$ & $0.0 \%$ & $0.0 \%$ & $0.0 \%$ & $0.0 \%$ \\
4 & $0.0 \%$ & $0.0 \%$ & $0.0 \%$ & $0.0 \%$ & $0.0 \%$ & $100.0 \%$ & $0.0 \%$ & $0.0 \%$ & $0.0 \%$ \\
5 & $-0.0 \%$ & $0.0 \%$ & $0.0 \%$ & $0.0 \%$ & $0.0 \%$ & $0.0 \%$ & $0.0 \%$ & $0.0 \%$ & $0.0 \%$ \\
6 & $-0.0 \%$ & $0.0 \%$ & $0.0 \%$ & $0.0 \%$ & $0.0 \%$ & $0.0 \%$ & $0.0 \%$ & $0.0 \%$ & $0.0 \%$ \\
7 & $48.7 \%$ & $48.7 \%$ & $0.5 \%$ & $0.5 \%$ & $0.6 \%$ & $0.6 \%$ & $0.1 \%$ & $0.1 \%$ & $0.1 \%$ \\
8 & $51.9 \%$ & $0.9 \%$ & $45.7 \%$ & $0.2 \%$ & $1.0 \%$ & $0.1 \%$ & $0.1 \%$ & $0.0 \%$ & $0.2 \%$ \\
9 & $48.2 \%$ & $0.5 \%$ & $0.4 \%$ & $0.1 \%$ & $49.7 \%$ & $0.1 \%$ & $0.4 \%$ & $0.0 \%$ & $0.4 \%$ \\
10 & $0.9 \%$ & $51.8 \%$ & $0.2 \%$ & $45.7 \%$ & $0.1 \%$ & $1.0 \%$ & $0.0 \%$ & $0.1 \%$ & $0.0 \%$ \\
11 & $0.5 \%$ & $48.2 \%$ & $0.1 \%$ & $0.4 \%$ & $0.1 \%$ & $49.7 \%$ & $0.0 \%$ & $0.4 \%$ & $0.0 \%$ \\
12 & $0.8 \%$ & $0.2 \%$ & $0.1 \%$ & $0.0 \%$ & $52.5 \%$ & $0.2 \%$ & $44.1 \%$ & $0.5 \%$ & $0.7 \%$ \\
13 & $0.9 \%$ & $0.1 \%$ & $0.2 \%$ & $0.0 \%$ & $52.7 \%$ & $0.0 \%$ & $0.6 \%$ & $0.0 \%$ & $45.1 \%$ \\
14 & $0.2 \%$ & $0.8 \%$ & $0.0 \%$ & $0.1 \%$ & $0.2 \%$ & $52.5 \%$ & $0.5 \%$ & $44.1 \%$ & $0.0 \%$ \\
15 & $0.1 \%$ & $0.9 \%$ & $0.0 \%$ & $0.2 \%$ & $0.0 \%$ & $52.7 \%$ & $0.0 \%$ & $0.6 \%$ & $0.0 \%$ \\
16 & $0.0 \%$ & $0.0 \%$ & $0.0 \%$ & $0.0 \%$ & $0.1 \%$ & $0.1 \%$ & $0.0 \%$ & $0.0 \%$ & $0.0 \%$ \\
17 & $0.1 \%$ & $0.0 \%$ & $0.0 \%$ & $0.0 \%$ & $0.5 \%$ & $0.0 \%$ & $0.3 \%$ & $0.0 \%$ & $0.0 \%$ \\
18 & $0.2 \%$ & $0.0 \%$ & $0.0 \%$ & $0.0 \%$ & $0.5 \%$ & $0.0 \%$ & $0.0 \%$ & $0.0 \%$ & $0.0 \%$ \\
19 & $0.0 \%$ & $0.1 \%$ & $0.0 \%$ & $0.0 \%$ & $0.0 \%$ & $0.5 \%$ & $0.0 \%$ & $0.3 \%$ & $0.0 \%$ \\
20 & $0.0 \%$ & $0.2 \%$ & $0.0 \%$ & $0.0 \%$ & $0.0 \%$ & $0.5 \%$ & $0.0 \%$ & $0.0 \%$ & $0.0 \%$ \\
21 & $45.8 \%$ & $15.7 \%$ & $1.6 \%$ & $0.4 \%$ & $30.4 \%$ & $0.9 \%$ & $0.8 \%$ & $0.0 \%$ & $1.0 \%$ \\
22 & $15.7 \%$ & $45.8 \%$ & $0.4 \%$ & $1.6 \%$ & $0.9 \%$ & $30.4 \%$ & $0.0 \%$ & $0.8 \%$ & $0.0 \%$ \\
23 & $0.8 \%$ & $0.8 \%$ & $0.1 \%$ & $0.1 \%$ & $4.3 \%$ & $4.3 \%$ & $0.5 \%$ & $0.5 \%$ & $0.1 \%$ \\
\hline \hline \hline
\end{tabular}

\begin{tabular}{crrrrrrrr}
\hline \hline \multicolumn{2}{c}{ Localization and atoms expanded by each FNO } \\
\hline FNO & Loc $_{10}$ & Loc $_{11}$ & Loc $_{12}$ & Loc $_{13}$ & Loc $_{14}$ & Loc $_{15}$ & Loc $_{16}$ & $n_{\text {eff }}$ \\
\hline 1 & $0.0 \%$ & $0.0 \%$ & $0.0 \%$ & $0.0 \%$ & $0.0 \%$ & $0.0 \%$ & $0.0 \%$ & 1.00002 \\
2 & $0.0 \%$ & $0.0 \%$ & $0.0 \%$ & $0.0 \%$ & $0.0 \%$ & $0.0 \%$ & $0.0 \%$ & 1.00002 \\
3 & $0.0 \%$ & $0.0 \%$ & $0.0 \%$ & $0.0 \%$ & $0.0 \%$ & $0.0 \%$ & $0.0 \%$ & 1.00002 \\
4 & $0.0 \%$ & $0.0 \%$ & $0.0 \%$ & $0.0 \%$ & $0.0 \%$ & $0.0 \%$ & $0.0 \%$ & 1.00002 \\
5 & $0.0 \%$ & $00.0 \%$ & $0.0 \%$ & $0.0 \%$ & $0.0 \%$ & $0.0 \%$ & $0.0 \%$ & 1.00002 \\
6 & $0.0 \%$ & $0.0 \%$ & $100.0 \%$ & $0.0 \%$ & $0.0 \%$ & $0.0 \%$ & $0.0 \%$ & 1.00002 \\
7 & $0.1 \%$ & $0.0 \%$ & $0.0 \%$ & $0.0 \%$ & $0.0 \%$ & $0.0 \%$ & $0.0 \%$ & 2.10627 \\
8 & $0.0 \%$ & $0.0 \%$ & $0.0 \%$ & $0.0 \%$ & $0.0 \%$ & $0.0 \%$ & $0.0 \%$ & 2.09358 \\
9 & $0.0 \%$ & $0.1 \%$ & $0.0 \%$ & $0.0 \%$ & $0.0 \%$ & $0.0 \%$ & $0.0 \%$ & 2.08698 \\
10 & $0.2 \%$ & $0.0 \%$ & $0.0 \%$ & $0.0 \%$ & $0.0 \%$ & $0.0 \%$ & $0.0 \%$ & 2.09425 \\
11 & $0.4 \%$ & $0.0 \%$ & $0.1 \%$ & $0.0 \%$ & $0.0 \%$ & $0.0 \%$ & $0.0 \%$ & 2.08673 \\
12 & $0.0 \%$ & $0.7 \%$ & $0.2 \%$ & $0.2 \%$ & $0.0 \%$ & $0.0 \%$ & $0.0 \%$ & 2.12809 \\
13 & $0.0 \%$ & $0.2 \%$ & $0.0 \%$ & $0.1 \%$ & $0.0 \%$ & $0.1 \%$ & $0.0 \%$ & 2.07753 \\
14 & $0.7 \%$ & $0.2 \%$ & $0.7 \%$ & $0.0 \%$ & $0.2 \%$ & $0.0 \%$ & $0.0 \%$ & 2.12793 \\
15 & $45.1 \%$ & $0.0 \%$ & $0.2 \%$ & $0.0 \%$ & $0.1 \%$ & $0.0 \%$ & $0.1 \%$ & 2.07726 \\
16 & $0.0 \%$ & $49.0 \%$ & $49.0 \%$ & $0.4 \%$ & $0.4 \%$ & $0.4 \%$ & $0.4 \%$ & 2.08202 \\
17 & $0.0 \%$ & $52.2 \%$ & $0.9 \%$ & $45.1 \%$ & $0.1 \%$ & $0.6 \%$ & $0.1 \%$ & 2.10282 \\
18 & $0.0 \%$ & $52.7 \%$ & $0.9 \%$ & $0.6 \%$ & $0.1 \%$ & $44.7 \%$ & $0.1 \%$ & 2.09463 \\
19 & $0.0 \%$ & $0.9 \%$ & $52.2 \%$ & $0.1 \%$ & $45.1 \%$ & $0.1 \%$ & $0.6 \%$ & 2.10265 \\
20 & $0.0 \%$ & $0.9 \%$ & $52.7 \%$ & $0.1 \%$ & $0.6 \%$ & $0.1 \%$ & $44.7 \%$ & 2.09534 \\
21 & $0.0 \%$ & $1.6 \%$ & $1.1 \%$ & $0.1 \%$ & $0.1 \%$ & $0.3 \%$ & $0.1 \%$ & 3.04943 \\
22 & $1.0 \%$ & $1.1 \%$ & $1.6 \%$ & $0.1 \%$ & $0.1 \%$ & $0.1 \%$ & $0.3 \%$ & 3.05044 \\
23 & $0.1 \%$ & $41.5 \%$ & $41.5 \%$ & $1.4 \%$ & $1.4 \%$ & $1.3 \%$ & $1.3 \%$ & 2.86184 \\
\hline \hline
\end{tabular}


S1.4.11 IRC Step 20

\begin{tabular}{|c|c|c|c|c|c|c|c|c|c|}
\hline \multicolumn{4}{|c|}{ 1-center FNO analysis, $\varepsilon=0.90000$} & & & & & & \\
\hline MO & $\lambda$ & \multicolumn{2}{|l|}{ Atoms } & & & & & & \\
\hline 1 & 0.99999 & \multicolumn{2}{|l|}{$\mathrm{C}_{1}$} & & & & & & \\
\hline 2 & 0.99999 & \multicolumn{2}{|l|}{$\mathrm{C}_{2}$} & & & & & & \\
\hline 3 & 0.99999 & \multicolumn{2}{|l|}{$\mathrm{C}_{5}$} & & & & & & \\
\hline 4 & 0.99999 & \multicolumn{2}{|l|}{$\mathrm{C}_{6}$} & & & & & & \\
\hline 5 & 0.99999 & \multicolumn{2}{|l|}{$\mathrm{C}_{11}$} & & & & & & \\
\hline 6 & 0.99999 & \multicolumn{2}{|l|}{$\mathrm{C}_{12}$} & & & & & & \\
\hline \multicolumn{10}{|c|}{ 2-center FNO analysis, $\varepsilon=0.90000$} \\
\hline MO & $\lambda$ & \multicolumn{3}{|l|}{ Atoms } & & & & & \\
\hline 7 & 0.97505 & \multicolumn{3}{|l|}{$\mathrm{C}_{1}-\mathrm{C}_{2}$} & & & & & \\
\hline 8 & 0.97550 & \multicolumn{3}{|l|}{$\mathrm{C}_{1}-\mathrm{H}_{3}$} & & & & & \\
\hline 9 & 0.97727 & \multicolumn{3}{|l|}{$\mathrm{C}_{1}-\mathrm{C}_{5}$} & & & & & \\
\hline 10 & 0.97536 & \multicolumn{3}{|l|}{$\mathrm{C}_{2}-\mathrm{H}_{4}$} & & & & & \\
\hline 11 & 0.97722 & \multicolumn{3}{|l|}{$\mathrm{C}_{2}-\mathrm{C}_{6}$} & & & & & \\
\hline 12 & 0.96436 & \multicolumn{3}{|l|}{$\mathrm{C}_{5}-\mathrm{H}_{7}$} & & & & & \\
\hline 13 & 0.97729 & $\mathrm{C}_{5}-\mathrm{H}_{9}$ & & & & & & & \\
\hline 14 & 0.96436 & $\mathrm{C}_{6}-\mathrm{H}_{8}$ & & & & & & & \\
\hline 15 & 0.97733 & $\mathrm{C}_{6}-\mathrm{H}_{10}$ & & & & & & & \\
\hline 16 & 0.97842 & $\mathrm{C}_{11}-\mathrm{C}_{12}$ & & & & & & & \\
\hline 17 & 0.97193 & $\mathrm{C}_{11}-\mathrm{C}_{12}$ & & & & & & & \\
\hline 18 & 0.97342 & $\mathrm{C}_{11}-\mathrm{H}_{13}$ & & & & & & & \\
\hline 19 & 0.97191 & $\mathrm{C}_{12}-\mathrm{H}_{14}$ & & & & & & & \\
\hline 20 & 0.97336 & $\mathrm{C}_{12}-\mathrm{H}_{16}$ & & & & & & & \\
\hline 3-cent & er FNO a & 1alysis, $\varepsilon=$ & $=0.90000$ & & & & & & \\
\hline $\mathrm{MO}$ & $\lambda$ & Atoms & & & & & & & \\
\hline 21 & 0.93232 & $\mathrm{C}_{1}-\mathrm{C}_{2}-$ & & & & & & & \\
\hline 22 & 0.93228 & $\mathrm{C}_{1}-\mathrm{C}_{2}-$ & & & & & & & \\
\hline 23 & 0.90608 & $\mathrm{C}_{5}-\mathrm{C}_{11}$ & & & & & & & \\
\hline 4-cent & er FNO a & 1alysis, $\varepsilon=$ & $=0.90000$ & & & & & & \\
\hline $\mathrm{MO}$ & $\lambda$ & Atoms & & & & & & & \\
\hline 23 & 0.90784 & $\mathrm{C}_{5}-\mathrm{C}_{6}-$ & $\mathrm{C}_{11}-\mathrm{C}_{12}$ & & & & & & \\
\hline$\overline{\text { Local }}$ & zation an & I atoms ex & $\overline{\overline{\text { panded } \mathrm{b}}}$ & y each & $\overline{\mathrm{NO}}$ & & & & \\
\hline $\mathrm{FNO}$ & $\mathrm{Loc}_{1}$ & $\mathrm{Loc}_{2}$ & $\mathrm{Loc}_{3}$ & $\mathrm{Loc}_{4}$ & $\mathrm{LOc}_{5}$ & $\mathrm{Loc}_{6}$ & $\mathrm{Loc}_{7}$ & $\mathrm{Loc}_{8}$ & $\mathrm{Loc}_{9}$ \\
\hline 1 & $100.0 \%$ & $0.0 \%$ & $0.0 \%$ & $0.0 \%$ & $0.0 \%$ & $0.0 \%$ & $0.0 \%$ & $0.0 \%$ & $0.0 \%$ \\
\hline 2 & $0.0 \%$ & $100.0 \%$ & $0.0 \%$ & $0.0 \%$ & $0.0 \%$ & $0.0 \%$ & $0.0 \%$ & $0.0 \%$ & $0.0 \%$ \\
\hline 3 & $0.0 \%$ & $0.0 \%$ & $0.0 \%$ & $0.0 \%$ & $100.0 \%$ & $0.0 \%$ & $0.0 \%$ & $0.0 \%$ & $0.0 \%$ \\
\hline 4 & $0.0 \%$ & $0.0 \%$ & $0.0 \%$ & $0.0 \%$ & $0.0 \%$ & $100.0 \%$ & $0.0 \%$ & $0.0 \%$ & $0.0 \%$ \\
\hline 5 & $-0.0 \%$ & $0.0 \%$ & $0.0 \%$ & $0.0 \%$ & $0.0 \%$ & $0.0 \%$ & $0.0 \%$ & $0.0 \%$ & $0.0 \%$ \\
\hline 6 & $-0.0 \%$ & $0.0 \%$ & $0.0 \%$ & $0.0 \%$ & $0.0 \%$ & $0.0 \%$ & $0.0 \%$ & $0.0 \%$ & $0.0 \%$ \\
\hline 7 & $48.7 \%$ & $48.8 \%$ & $0.5 \%$ & $0.5 \%$ & $0.6 \%$ & $0.6 \%$ & $0.1 \%$ & $0.1 \%$ & $0.1 \%$ \\
\hline 8 & $51.9 \%$ & $0.9 \%$ & $45.6 \%$ & $0.2 \%$ & $1.0 \%$ & $0.1 \%$ & $0.1 \%$ & $0.0 \%$ & $0.1 \%$ \\
\hline 9 & $48.1 \%$ & $0.6 \%$ & $0.4 \%$ & $0.1 \%$ & $49.6 \%$ & $0.1 \%$ & $0.4 \%$ & $0.0 \%$ & $0.5 \%$ \\
\hline 10 & $0.9 \%$ & $51.9 \%$ & $0.2 \%$ & $45.6 \%$ & $0.1 \%$ & $1.0 \%$ & $0.0 \%$ & $0.1 \%$ & $0.0 \%$ \\
\hline 11 & $0.6 \%$ & $48.1 \%$ & $0.1 \%$ & $0.4 \%$ & $0.1 \%$ & $49.6 \%$ & $0.0 \%$ & $0.4 \%$ & $0.0 \%$ \\
\hline 12 & $0.9 \%$ & $0.2 \%$ & $0.1 \%$ & $0.0 \%$ & $52.3 \%$ & $0.2 \%$ & $44.2 \%$ & $0.5 \%$ & $0.7 \%$ \\
\hline 13 & $0.9 \%$ & $0.1 \%$ & $0.1 \%$ & $0.0 \%$ & $52.5 \%$ & $0.0 \%$ & $0.6 \%$ & $0.0 \%$ & $45.2 \%$ \\
\hline 14 & $0.2 \%$ & $0.9 \%$ & $0.0 \%$ & $0.1 \%$ & $0.2 \%$ & $52.3 \%$ & $0.5 \%$ & $44.2 \%$ & $0.0 \%$ \\
\hline 15 & $0.1 \%$ & $0.8 \%$ & $0.0 \%$ & $0.2 \%$ & $0.0 \%$ & $52.5 \%$ & $0.0 \%$ & $0.6 \%$ & $0.0 \%$ \\
\hline 16 & $0.0 \%$ & $0.0 \%$ & $0.0 \%$ & $0.0 \%$ & $0.1 \%$ & $0.1 \%$ & $0.0 \%$ & $0.0 \%$ & $0.0 \%$ \\
\hline 17 & $0.1 \%$ & $0.0 \%$ & $0.0 \%$ & $0.0 \%$ & $0.6 \%$ & $0.0 \%$ & $0.2 \%$ & $0.0 \%$ & $0.0 \%$ \\
\hline 18 & $0.1 \%$ & $0.0 \%$ & $0.0 \%$ & $0.0 \%$ & $0.6 \%$ & $0.0 \%$ & $0.0 \%$ & $0.0 \%$ & $0.1 \%$ \\
\hline 19 & $0.0 \%$ & $0.1 \%$ & $0.0 \%$ & $0.0 \%$ & $0.0 \%$ & $0.6 \%$ & $0.0 \%$ & $0.2 \%$ & $0.0 \%$ \\
\hline 20 & $-0.0 \%$ & $0.2 \%$ & $0.0 \%$ & $0.0 \%$ & $0.0 \%$ & $0.6 \%$ & $0.0 \%$ & $0.0 \%$ & $0.0 \%$ \\
\hline 21 & $45.6 \%$ & $21.4 \%$ & $1.6 \%$ & $0.7 \%$ & $24.4 \%$ & $1.4 \%$ & $0.7 \%$ & $0.1 \%$ & $0.7 \%$ \\
\hline 22 & $21.4 \%$ & $45.6 \%$ & $0.7 \%$ & $1.6 \%$ & $1.4 \%$ & $24.4 \%$ & $0.1 \%$ & $0.7 \%$ & $0.0 \%$ \\
\hline 23 & $0.9 \%$ & $0.9 \%$ & $0.1 \%$ & $0.1 \%$ & $6.8 \%$ & $6.8 \%$ & $0.6 \%$ & $0.6 \%$ & $0.2 \%$ \\
\hline
\end{tabular}




\begin{tabular}{crrrrrrrr}
\hline \multicolumn{2}{l}{ localization and atoms expanded by each FNO } \\
\hline FNO & Loc $_{10}$ & Loc $_{11}$ & Loc $_{12}$ & Loc $_{13}$ & Loc $_{14}$ & Loc $_{15}$ & Loc $_{16}$ & $n_{\text {eff }}$ \\
\hline 1 & $0.0 \%$ & $0.0 \%$ & $0.0 \%$ & $0.0 \%$ & $0.0 \%$ & $0.0 \%$ & $0.0 \%$ & 1.00002 \\
2 & $0.0 \%$ & $0.0 \%$ & $0.0 \%$ & $0.0 \%$ & $0.0 \%$ & $0.0 \%$ & $0.0 \%$ & 1.00002 \\
3 & $0.0 \%$ & $0.0 \%$ & $0.0 \%$ & $0.0 \%$ & $0.0 \%$ & $0.0 \%$ & $0.0 \%$ & 1.00002 \\
4 & $0.0 \%$ & $0.0 \%$ & $0.0 \%$ & $0.0 \%$ & $0.0 \%$ & $0.0 \%$ & $0.0 \%$ & 1.00002 \\
5 & $0.0 \%$ & $100.0 \%$ & $0.0 \%$ & $0.0 \%$ & $0.0 \%$ & $0.0 \%$ & $0.0 \%$ & 1.00002 \\
6 & $0.0 \%$ & $0.0 \%$ & $100.0 \%$ & $0.0 \%$ & $0.0 \%$ & $0.0 \%$ & $0.0 \%$ & 1.00002 \\
7 & $0.1 \%$ & $0.0 \%$ & $0.0 \%$ & $0.0 \%$ & $0.0 \%$ & $0.0 \%$ & $0.0 \%$ & 2.10313 \\
8 & $0.0 \%$ & $0.0 \%$ & $0.0 \%$ & $0.0 \%$ & $0.0 \%$ & $0.0 \%$ & $0.0 \%$ & 2.09229 \\
9 & $0.0 \%$ & $0.2 \%$ & $0.0 \%$ & $0.0 \%$ & $0.0 \%$ & $0.0 \%$ & $0.0 \%$ & 2.09322 \\
10 & $0.2 \%$ & $0.0 \%$ & $0.0 \%$ & $0.0 \%$ & $0.0 \%$ & $0.0 \%$ & $0.0 \%$ & 2.09292 \\
11 & $0.5 \%$ & $0.0 \%$ & $0.2 \%$ & $0.0 \%$ & $0.0 \%$ & $0.0 \%$ & $0.0 \%$ & 2.09339 \\
12 & $0.0 \%$ & $0.7 \%$ & $0.2 \%$ & $0.2 \%$ & $0.0 \%$ & $0.0 \%$ & $0.0 \%$ & 2.13460 \\
13 & $0.0 \%$ & $0.3 \%$ & $0.0 \%$ & $0.1 \%$ & $0.0 \%$ & $0.1 \%$ & $0.0 \%$ & 2.08174 \\
14 & $0.7 \%$ & $0.2 \%$ & $0.7 \%$ & $0.0 \%$ & $0.2 \%$ & $0.0 \%$ & $0.0 \%$ & 2.13458 \\
15 & $45.2 \%$ & $0.0 \%$ & $0.3 \%$ & $0.0 \%$ & $0.1 \%$ & $0.0 \%$ & $0.1 \%$ & 2.08156 \\
16 & $0.0 \%$ & $48.9 \%$ & $48.9 \%$ & $0.4 \%$ & $0.4 \%$ & $0.4 \%$ & $0.4 \%$ & 2.08885 \\
17 & $0.0 \%$ & $52.2 \%$ & $0.9 \%$ & $45.0 \%$ & $0.1 \%$ & $0.6 \%$ & $0.1 \%$ & 2.10471 \\
18 & $0.0 \%$ & $52.6 \%$ & $0.9 \%$ & $0.6 \%$ & $0.1 \%$ & $44.7 \%$ & $0.1 \%$ & 2.09599 \\
19 & $0.0 \%$ & $0.9 \%$ & $52.2 \%$ & $0.1 \%$ & $45.0 \%$ & $0.1 \%$ & $0.6 \%$ & 2.10481 \\
20 & $0.1 \%$ & $0.9 \%$ & $52.6 \%$ & $0.1 \%$ & $0.6 \%$ & $0.1 \%$ & $44.7 \%$ & 2.09626 \\
21 & $0.0 \%$ & $1.6 \%$ & $1.3 \%$ & $0.1 \%$ & $0.1 \%$ & $0.3 \%$ & $0.1 \%$ & 3.17910 \\
22 & $0.7 \%$ & $1.3 \%$ & $1.6 \%$ & $0.1 \%$ & $0.1 \%$ & $0.1 \%$ & $0.3 \%$ & 3.18005 \\
23 & $0.2 \%$ & $38.8 \%$ & $38.8 \%$ & $1.3 \%$ & $1.3 \%$ & $1.3 \%$ & $1.3 \%$ & 3.21744 \\
\hline \hline
\end{tabular}

\section{S1.4.12 IRC Step 22}

\begin{tabular}{|c|c|c|}
\hline \multicolumn{3}{|c|}{1 1-center FNO analysis, $\varepsilon=0.90000$} \\
\hline $\mathrm{MO}$ & $\lambda$ & Atoms \\
\hline 1 & 0.99999 & $\mathrm{C}_{1}$ \\
\hline 2 & 0.99999 & $\mathrm{C}_{2}$ \\
\hline 3 & 0.99999 & $\mathrm{C}_{5}$ \\
\hline 4 & 0.99999 & $\mathrm{C}_{6}$ \\
\hline 5 & 0.99999 & $\mathrm{C}_{11}$ \\
\hline 6 & 0.99999 & $\mathrm{C}_{12}$ \\
\hline \multicolumn{3}{|c|}{ 2-center FNO analysis, $\varepsilon=0.90000$} \\
\hline MO & $\lambda$ & Atoms \\
\hline 7 & 0.97686 & $\mathrm{C}_{1}-\mathrm{C}_{2}$ \\
\hline 8 & 0.97677 & $\mathrm{C}_{1}-\mathrm{H}_{3}$ \\
\hline 9 & 0.97345 & $\mathrm{C}_{1}-\mathrm{C}_{5}$ \\
\hline 10 & 0.97661 & $\mathrm{C}_{2}-\mathrm{H}_{4}$ \\
\hline 11 & 0.97333 & $\mathrm{C}_{2}-\mathrm{C}_{6}$ \\
\hline 12 & 0.96285 & $\mathrm{C}_{5}-\mathrm{H}_{7}$ \\
\hline 13 & 0.97544 & $\mathrm{C}_{5}-\mathrm{H}_{9}$ \\
\hline 14 & 0.96297 & $\mathrm{C}_{6}-\mathrm{H}_{8}$ \\
\hline 15 & 0.97541 & $\mathrm{C}_{6}-\mathrm{H}_{10}$ \\
\hline 16 & 0.97323 & $\mathrm{C}_{11}-\mathrm{C}_{12}$ \\
\hline 17 & 0.97117 & $\mathrm{C}_{11}-\mathrm{H}_{13}$ \\
\hline 18 & 0.97220 & $\mathrm{C}_{11}-\mathrm{H}_{15}$ \\
\hline 19 & 0.97119 & $\mathrm{C}_{12}-\mathrm{H}_{14}$ \\
\hline 20 & 0.97224 & $\mathrm{C}_{12}-\mathrm{H}_{16}$ \\
\hline \multicolumn{3}{|c|}{3 -center FNO analysis, $\varepsilon=0.90000$} \\
\hline $\mathrm{MO}$ & $\lambda$ & Atoms \\
\hline 21 & 0.91509 & $\mathrm{C}_{1}-\mathrm{C}_{2}-\mathrm{C}_{5}$ \\
\hline 22 & 0.91503 & $\mathrm{C}_{1}-\mathrm{C}_{2}-\mathrm{C}_{6}$ \\
\hline 23 & 0.90097 & $\mathrm{C}_{1}-\mathrm{C}_{5}-\mathrm{C}_{11}$ \\
\hline
\end{tabular}




\begin{tabular}{crrrrrrrrr}
\hline \multicolumn{2}{l}{ Localization and atoms expanded by each FNO } \\
\hline FNO & Loc $_{1}$ & Loc $_{2}$ & \multicolumn{1}{l}{$\mathrm{Loc}_{3}$} & $\mathrm{Loc}_{4}$ & $\mathrm{Loc}_{5}$ & $\mathrm{Loc}_{6}$ & $\mathrm{Loc}_{7}$ & $\mathrm{Loc}_{8}$ & $\mathrm{Loc}_{9}$ \\
\hline 1 & $100.0 \%$ & $0.0 \%$ & $0.0 \%$ & $0.0 \%$ & $0.0 \%$ & $0.0 \%$ & $0.0 \%$ & $0.0 \%$ & $0.0 \%$ \\
2 & $0.0 \%$ & $100.0 \%$ & $0.0 \%$ & $0.0 \%$ & $0.0 \%$ & $0.0 \%$ & $0.0 \%$ & $0.0 \%$ & $0.0 \%$ \\
3 & $0.0 \%$ & $0.0 \%$ & $0.0 \%$ & $0.0 \%$ & $100.0 \%$ & $0.0 \%$ & $0.0 \%$ & $0.0 \%$ & $0.0 \%$ \\
4 & $0.0 \%$ & $0.0 \%$ & $0.0 \%$ & $0.0 \%$ & $0.0 \%$ & $100.0 \%$ & $0.0 \%$ & $0.0 \%$ & $0.0 \%$ \\
5 & $-0.0 \%$ & $0.0 \%$ & $0.0 \%$ & $0.0 \%$ & $0.0 \%$ & $0.0 \%$ & $0.0 \%$ & $0.0 \%$ & $0.0 \%$ \\
6 & $-0.0 \%$ & $0.0 \%$ & $0.0 \%$ & $0.0 \%$ & $0.0 \%$ & $0.0 \%$ & $0.0 \%$ & $0.0 \%$ & $0.0 \%$ \\
7 & $48.8 \%$ & $48.8 \%$ & $0.5 \%$ & $0.5 \%$ & $0.6 \%$ & $0.6 \%$ & $0.1 \%$ & $0.1 \%$ & $0.1 \%$ \\
8 & $52.1 \%$ & $0.9 \%$ & $45.5 \%$ & $0.2 \%$ & $0.9 \%$ & $0.1 \%$ & $0.1 \%$ & $0.0 \%$ & $0.1 \%$ \\
9 & $48.3 \%$ & $0.6 \%$ & $0.5 \%$ & $0.1 \%$ & $49.0 \%$ & $0.1 \%$ & $0.5 \%$ & $0.0 \%$ & $0.5 \%$ \\
10 & $0.9 \%$ & $52.1 \%$ & $0.2 \%$ & $45.5 \%$ & $0.1 \%$ & $0.9 \%$ & $0.0 \%$ & $0.1 \%$ & $0.0 \%$ \\
11 & $0.6 \%$ & $48.3 \%$ & $0.1 \%$ & $0.5 \%$ & $0.1 \%$ & $49.0 \%$ & $0.0 \%$ & $0.5 \%$ & $0.0 \%$ \\
12 & $1.0 \%$ & $0.2 \%$ & $0.1 \%$ & $0.0 \%$ & $51.6 \%$ & $0.2 \%$ & $44.7 \%$ & $0.4 \%$ & $0.7 \%$ \\
13 & $0.9 \%$ & $0.1 \%$ & $0.1 \%$ & $0.0 \%$ & $51.9 \%$ & $0.0 \%$ & $0.7 \%$ & $0.0 \%$ & $45.6 \%$ \\
14 & $0.2 \%$ & $1.0 \%$ & $0.0 \%$ & $0.1 \%$ & $0.2 \%$ & $51.6 \%$ & $0.4 \%$ & $44.7 \%$ & $0.0 \%$ \\
15 & $0.1 \%$ & $0.9 \%$ & $0.0 \%$ & $0.1 \%$ & $0.0 \%$ & $51.9 \%$ & $0.0 \%$ & $0.7 \%$ & $0.0 \%$ \\
16 & $0.1 \%$ & $0.0 \%$ & $0.0 \%$ & $0.0 \%$ & $0.3 \%$ & $0.3 \%$ & $0.0 \%$ & $0.0 \%$ & $0.0 \%$ \\
17 & $0.1 \%$ & $0.0 \%$ & $0.0 \%$ & $0.0 \%$ & $0.6 \%$ & $0.1 \%$ & $0.1 \%$ & $0.0 \%$ & $0.0 \%$ \\
18 & $0.2 \%$ & $0.0 \%$ & $0.0 \%$ & $0.0 \%$ & $0.6 \%$ & $0.1 \%$ & $0.0 \%$ & $0.0 \%$ & $0.1 \%$ \\
19 & $0.0 \%$ & $0.1 \%$ & $0.0 \%$ & $0.0 \%$ & $0.1 \%$ & $0.6 \%$ & $0.0 \%$ & $0.1 \%$ & $0.0 \%$ \\
20 & $0.0 \%$ & $0.1 \%$ & $0.0 \%$ & $0.0 \%$ & $0.1 \%$ & $0.6 \%$ & $0.0 \%$ & $0.0 \%$ & $0.0 \%$ \\
21 & $45.6 \%$ & $34.2 \%$ & $1.6 \%$ & $1.2 \%$ & $11.7 \%$ & $2.2 \%$ & $0.3 \%$ & $0.2 \%$ & $0.3 \%$ \\
22 & $34.2 \%$ & $45.6 \%$ & $1.2 \%$ & $1.6 \%$ & $2.2 \%$ & $11.7 \%$ & $0.2 \%$ & $0.3 \%$ & $0.0 \%$ \\
23 & $14.8 \%$ & $0.9 \%$ & $0.4 \%$ & $0.0 \%$ & $43.5 \%$ & $0.9 \%$ & $1.5 \%$ & $0.0 \%$ & $1.4 \%$ \\
\hline \hline \hline
\end{tabular}

\begin{tabular}{crrrrrrrr}
\hline \hline \multicolumn{2}{c}{ Localization and atoms expanded by each FNO } \\
\hline FNO & Loc $_{10}$ & Loc $_{11}$ & Loc $_{12}$ & Loc $_{13}$ & Loc $_{14}$ & Loc $_{15}$ & Loc $_{16}$ & $n_{\text {eff }}$ \\
\hline 1 & $0.0 \%$ & $0.0 \%$ & $0.0 \%$ & $0.0 \%$ & $0.0 \%$ & $0.0 \%$ & $0.0 \%$ & 1.00002 \\
2 & $0.0 \%$ & $0.0 \%$ & $0.0 \%$ & $0.0 \%$ & $0.0 \%$ & $0.0 \%$ & $0.0 \%$ & 1.00002 \\
3 & $0.0 \%$ & $0.0 \%$ & $0.0 \%$ & $0.0 \%$ & $0.0 \%$ & $0.0 \%$ & $0.0 \%$ & 1.00002 \\
4 & $0.0 \%$ & $0.0 \%$ & $0.0 \%$ & $0.0 \%$ & $0.0 \%$ & $0.0 \%$ & $0.0 \%$ & 1.00002 \\
5 & $0.0 \%$ & $100.0 \%$ & $0.0 \%$ & $0.0 \%$ & $0.0 \%$ & $0.0 \%$ & $0.0 \%$ & 1.00002 \\
6 & $0.0 \%$ & $0.0 \%$ & $100.0 \%$ & $0.0 \%$ & $0.0 \%$ & $0.0 \%$ & $0.0 \%$ & 1.00002 \\
7 & $0.1 \%$ & $0.0 \%$ & $0.0 \%$ & $0.0 \%$ & $0.0 \%$ & $0.0 \%$ & $0.0 \%$ & 2.09542 \\
8 & $0.0 \%$ & $0.0 \%$ & $0.0 \%$ & $0.0 \%$ & $0.0 \%$ & $0.0 \%$ & $0.0 \%$ & 2.08597 \\
9 & $0.0 \%$ & $0.2 \%$ & $0.0 \%$ & $0.0 \%$ & $0.0 \%$ & $0.0 \%$ & $0.0 \%$ & 2.10990 \\
10 & $0.2 \%$ & $0.0 \%$ & $0.0 \%$ & $0.0 \%$ & $0.0 \%$ & $0.0 \%$ & $0.0 \%$ & 2.08668 \\
11 & $0.5 \%$ & $0.0 \%$ & $0.2 \%$ & $0.0 \%$ & $0.0 \%$ & $0.0 \%$ & $0.0 \%$ & 2.11044 \\
12 & $0.0 \%$ & $0.7 \%$ & $0.1 \%$ & $0.1 \%$ & $0.0 \%$ & $0.0 \%$ & $0.0 \%$ & 2.14526 \\
13 & $0.0 \%$ & $0.4 \%$ & $0.0 \%$ & $0.0 \%$ & $0.0 \%$ & $0.1 \%$ & $0.0 \%$ & 2.09252 \\
14 & $0.7 \%$ & $0.1 \%$ & $0.7 \%$ & $0.0 \%$ & $0.1 \%$ & $0.0 \%$ & $0.0 \%$ & 2.14462 \\
15 & $45.6 \%$ & $0.0 \%$ & $0.4 \%$ & $0.0 \%$ & $0.0 \%$ & $0.0 \%$ & $0.1 \%$ & 2.09266 \\
16 & $0.0 \%$ & $48.7 \%$ & $48.7 \%$ & $0.5 \%$ & $0.5 \%$ & $0.5 \%$ & $0.5 \%$ & 2.11106 \\
17 & $0.0 \%$ & $51.8 \%$ & $1.0 \%$ & $45.3 \%$ & $0.2 \%$ & $0.7 \%$ & $0.1 \%$ & 2.11007 \\
18 & $0.0 \%$ & $52.1 \%$ & $0.9 \%$ & $0.7 \%$ & $0.1 \%$ & $45.1 \%$ & $0.2 \%$ & 2.10454 \\
19 & $0.0 \%$ & $1.0 \%$ & $51.8 \%$ & $0.2 \%$ & $45.3 \%$ & $0.1 \%$ & $0.7 \%$ & 2.10997 \\
20 & $0.1 \%$ & $0.9 \%$ & $52.1 \%$ & $0.1 \%$ & $0.7 \%$ & $0.2 \%$ & $45.1 \%$ & 2.10434 \\
21 & $0.0 \%$ & $1.0 \%$ & $1.3 \%$ & $0.0 \%$ & $0.1 \%$ & $0.2 \%$ & $0.1 \%$ & 2.93923 \\
22 & $0.3 \%$ & $1.3 \%$ & $1.0 \%$ & $0.1 \%$ & $0.0 \%$ & $0.1 \%$ & $0.2 \%$ & 2.93971 \\
23 & $0.1 \%$ & $32.1 \%$ & $1.7 \%$ & $1.1 \%$ & $0.1 \%$ & $1.3 \%$ & $0.1 \%$ & 3.17282 \\
\hline \hline
\end{tabular}


S1.4.13 IRC Step 24

\begin{tabular}{|c|c|c|c|c|c|c|c|c|c|}
\hline \multicolumn{4}{|c|}{ 1-center FNO analysis, $\varepsilon=0.90000$} & & & & & & \\
\hline MO & $\lambda$ & \multicolumn{2}{|l|}{ Atoms } & & & & & & \\
\hline 1 & 0.99999 & \multicolumn{2}{|l|}{$\mathrm{C}_{1}$} & & & & & & \\
\hline 2 & 0.99999 & \multicolumn{2}{|l|}{$\mathrm{C}_{2}$} & & & & & & \\
\hline 3 & 0.99999 & \multicolumn{2}{|l|}{$\mathrm{C}_{5}$} & & & & & & \\
\hline 4 & 0.99999 & \multicolumn{2}{|l|}{$\mathrm{C}_{6}$} & & & & & & \\
\hline 5 & 0.99999 & \multicolumn{2}{|l|}{$\mathrm{C}_{11}$} & & & & & & \\
\hline 6 & 0.99999 & \multicolumn{2}{|l|}{$\mathrm{C}_{12}$} & & & & & & \\
\hline \multicolumn{10}{|c|}{ 2-center FNO analysis, $\varepsilon=0.90000$} \\
\hline $\mathrm{MO}$ & $\lambda$ & \multicolumn{3}{|c|}{ Atoms } & & & & & \\
\hline 7 & 0.97844 & \multicolumn{3}{|l|}{$\mathrm{C}_{1}-\mathrm{C}_{2}$} & & & & & \\
\hline 8 & 0.97861 & \multicolumn{3}{|l|}{$\mathrm{C}_{1}-\mathrm{H}_{3}$} & & & & & \\
\hline 9 & 0.96855 & \multicolumn{3}{|l|}{$\mathrm{C}_{1}-\mathrm{C}_{5}$} & & & & & \\
\hline 10 & 0.97843 & \multicolumn{3}{|l|}{$\mathrm{C}_{2}-\mathrm{H}_{4}$} & & & & & \\
\hline 11 & 0.96851 & \multicolumn{3}{|l|}{$\mathrm{C}_{2}-\mathrm{C}_{6}$} & & & & & \\
\hline 12 & 0.95951 & \multicolumn{3}{|l|}{$\mathrm{C}_{5}-\mathrm{H}_{7}$} & & & & & \\
\hline 13 & 0.97217 & \multicolumn{3}{|l|}{$\mathrm{C}_{5}-\mathrm{H}_{9}$} & & & & & \\
\hline 14 & 0.90868 & $\mathrm{C}_{5}-\mathrm{H}_{11}$ & & & & & & & \\
\hline 15 & 0.95962 & $\mathrm{C}_{6}-\mathrm{H}_{8}$ & & & & & & & \\
\hline 16 & 0.97219 & $\mathrm{C}_{6}-\mathrm{C}_{10}$ & & & & & & & \\
\hline 17 & 0.90862 & $\mathrm{C}_{6}-\mathrm{C}_{12}$ & & & & & & & \\
\hline 18 & 0.96506 & $\mathrm{C}_{11}-\mathrm{C}_{12}$ & & & & & & & \\
\hline 19 & 0.96967 & $\mathrm{C}_{11}-\mathrm{H}_{13}$ & & & & & & & \\
\hline 20 & 0.97061 & $\mathrm{C}_{11}-\mathrm{H}_{15}$ & & & & & & & \\
\hline 21 & 0.96953 & $\mathrm{C}_{12}-\mathrm{H}_{14}$ & & & & & & & \\
\hline 22 & 0.97043 & $\mathrm{C}_{12}-\mathrm{H}_{16}$ & & & & & & & \\
\hline 3-cen & er FNO a & talysis, $\varepsilon$ & $=0.90000$ & & & & & & \\
\hline $\mathrm{MO}$ & $\lambda$ & Atoms & & & & & & & \\
\hline 23 & 0.90649 & $\mathrm{C}_{1}-\mathrm{C}_{2}-$ & & & & & & & \\
\hline Local & zation an & I atoms ex & panded & y each & $\overline{\mathrm{NO}}$ & & & & \\
\hline FNO & $\mathrm{Loc}_{1}$ & $\mathrm{Loc}_{2}$ & $\mathrm{Loc}_{3}$ & $\mathrm{Loc}_{4}$ & $\mathrm{Loc}_{5}$ & $\mathrm{LOc}_{6}$ & $\mathrm{Loc}_{7}$ & $\mathrm{Loc}_{8}$ & $\mathrm{Loc}_{9}$ \\
\hline 1 & $100.0 \%$ & $0.0 \%$ & $0.0 \%$ & $0.0 \%$ & $0.0 \%$ & $0.0 \%$ & $0.0 \%$ & $0.0 \%$ & $0.0 \%$ \\
\hline 2 & $0.0 \%$ & $100.0 \%$ & $0.0 \%$ & $0.0 \%$ & $0.0 \%$ & $0.0 \%$ & $0.0 \%$ & $0.0 \%$ & $0.0 \%$ \\
\hline 3 & $0.0 \%$ & $0.0 \%$ & $0.0 \%$ & $0.0 \%$ & $100.0 \%$ & $0.0 \%$ & $0.0 \%$ & $0.0 \%$ & $0.0 \%$ \\
\hline 4 & $-0.0 \%$ & $0.0 \%$ & $0.0 \%$ & $0.0 \%$ & $0.0 \%$ & $100.0 \%$ & $0.0 \%$ & $0.0 \%$ & $0.0 \%$ \\
\hline 5 & $0.0 \%$ & $0.0 \%$ & $0.0 \%$ & $0.0 \%$ & $0.0 \%$ & $0.0 \%$ & $0.0 \%$ & $0.0 \%$ & $0.0 \%$ \\
\hline 6 & $0.0 \%$ & $0.0 \%$ & $0.0 \%$ & $0.0 \%$ & $0.0 \%$ & $0.0 \%$ & $0.0 \%$ & $0.0 \%$ & $0.0 \%$ \\
\hline 7 & $48.9 \%$ & $48.9 \%$ & $0.4 \%$ & $0.4 \%$ & $0.5 \%$ & $0.5 \%$ & $0.0 \%$ & $0.0 \%$ & $0.1 \%$ \\
\hline 8 & $52.5 \%$ & $0.9 \%$ & $45.3 \%$ & $0.1 \%$ & $0.8 \%$ & $0.1 \%$ & $0.0 \%$ & $0.0 \%$ & $0.2 \%$ \\
\hline 9 & $49.0 \%$ & $0.7 \%$ & $0.5 \%$ & $0.1 \%$ & $47.9 \%$ & $0.2 \%$ & $0.6 \%$ & $0.0 \%$ & $0.6 \%$ \\
\hline 10 & $0.9 \%$ & $52.5 \%$ & $0.1 \%$ & $45.3 \%$ & $0.1 \%$ & $0.8 \%$ & $0.0 \%$ & $0.0 \%$ & $0.0 \%$ \\
\hline 11 & $0.7 \%$ & $49.0 \%$ & $0.1 \%$ & $0.5 \%$ & $0.2 \%$ & $47.9 \%$ & $0.0 \%$ & $0.6 \%$ & $0.0 \%$ \\
\hline 12 & $1.2 \%$ & $0.3 \%$ & $0.1 \%$ & $0.0 \%$ & $50.4 \%$ & $0.2 \%$ & $45.5 \%$ & $0.4 \%$ & $0.8 \%$ \\
\hline 13 & $0.9 \%$ & $0.1 \%$ & $0.1 \%$ & $0.0 \%$ & $50.9 \%$ & $0.0 \%$ & $0.8 \%$ & $0.0 \%$ & $46.3 \%$ \\
\hline 14 & $2.0 \%$ & $0.7 \%$ & $0.0 \%$ & $0.1 \%$ & $45.7 \%$ & $0.4 \%$ & $1.1 \%$ & $0.1 \%$ & $1.0 \%$ \\
\hline 15 & $0.3 \%$ & $1.1 \%$ & $0.0 \%$ & $0.1 \%$ & $0.2 \%$ & $50.4 \%$ & $0.4 \%$ & $45.5 \%$ & $0.0 \%$ \\
\hline 16 & $0.1 \%$ & $0.9 \%$ & $0.0 \%$ & $0.1 \%$ & $0.0 \%$ & $50.9 \%$ & $0.0 \%$ & $0.8 \%$ & $0.0 \%$ \\
\hline 17 & $0.7 \%$ & $2.0 \%$ & $0.1 \%$ & $0.0 \%$ & $0.4 \%$ & $45.7 \%$ & $0.1 \%$ & $1.1 \%$ & $0.0 \%$ \\
\hline 18 & $0.1 \%$ & $0.1 \%$ & $0.0 \%$ & $0.0 \%$ & $0.4 \%$ & $0.4 \%$ & $0.0 \%$ & $0.0 \%$ & $0.0 \%$ \\
\hline 19 & $0.1 \%$ & $0.0 \%$ & $0.0 \%$ & $0.0 \%$ & $0.7 \%$ & $0.0 \%$ & $0.1 \%$ & $0.0 \%$ & $0.0 \%$ \\
\hline 20 & $0.2 \%$ & $0.0 \%$ & $0.0 \%$ & $0.0 \%$ & $0.7 \%$ & $0.0 \%$ & $0.1 \%$ & $0.0 \%$ & $0.1 \%$ \\
\hline 21 & $0.1 \%$ & $0.1 \%$ & $0.0 \%$ & $0.0 \%$ & $0.0 \%$ & $0.7 \%$ & $0.0 \%$ & $0.1 \%$ & $0.0 \%$ \\
\hline 22 & $0.0 \%$ & $0.1 \%$ & $0.0 \%$ & $0.0 \%$ & $0.0 \%$ & $0.7 \%$ & $0.0 \%$ & $0.1 \%$ & $0.0 \%$ \\
\hline 23 & $44.6 \%$ & $44.4 \%$ & $1.7 \%$ & $1.7 \%$ & $2.4 \%$ & $2.4 \%$ & $0.3 \%$ & $0.3 \%$ & $0.0 \%$ \\
\hline
\end{tabular}




\begin{tabular}{crrrrrrrr}
\hline \multicolumn{2}{l}{ localization and atoms expanded by each FNO } \\
\hline FNO & Loc $_{10}$ & Loc $_{11}$ & Loc $_{12}$ & Loc $_{13}$ & Loc $_{14}$ & Loc $_{15}$ & Loc $_{16}$ & $n_{\text {eff }}$ \\
\hline 1 & $0.0 \%$ & $0.0 \%$ & $0.0 \%$ & $0.0 \%$ & $0.0 \%$ & $0.0 \%$ & $0.0 \%$ & 1.00002 \\
2 & $0.0 \%$ & $0.0 \%$ & $0.0 \%$ & $0.0 \%$ & $0.0 \%$ & $0.0 \%$ & $0.0 \%$ & 1.00002 \\
3 & $0.0 \%$ & $0.0 \%$ & $0.0 \%$ & $0.0 \%$ & $0.0 \%$ & $0.0 \%$ & $0.0 \%$ & 1.00002 \\
4 & $0.0 \%$ & $0.0 \%$ & $0.0 \%$ & $0.0 \%$ & $0.0 \%$ & $0.0 \%$ & $0.0 \%$ & 1.00002 \\
5 & $0.0 \%$ & $100.0 \%$ & $0.0 \%$ & $0.0 \%$ & $0.0 \%$ & $0.0 \%$ & $0.0 \%$ & 1.00001 \\
6 & $0.0 \%$ & $0.0 \%$ & $100.0 \%$ & $0.0 \%$ & $0.0 \%$ & $0.0 \%$ & $0.0 \%$ & 1.00001 \\
7 & $0.1 \%$ & $0.0 \%$ & $0.0 \%$ & $0.0 \%$ & $0.0 \%$ & $0.0 \%$ & $0.0 \%$ & 2.08871 \\
8 & $0.0 \%$ & $0.0 \%$ & $0.0 \%$ & $0.0 \%$ & $0.0 \%$ & $0.0 \%$ & $0.0 \%$ & 2.07660 \\
9 & $0.0 \%$ & $0.4 \%$ & $0.0 \%$ & $0.0 \%$ & $0.0 \%$ & $0.0 \%$ & $0.0 \%$ & 2.13094 \\
10 & $0.2 \%$ & $0.0 \%$ & $0.0 \%$ & $0.0 \%$ & $0.0 \%$ & $0.0 \%$ & $0.0 \%$ & 2.07741 \\
11 & $0.6 \%$ & $0.0 \%$ & $0.4 \%$ & $0.0 \%$ & $0.0 \%$ & $0.0 \%$ & $0.0 \%$ & 2.13114 \\
12 & $0.0 \%$ & $0.7 \%$ & $0.1 \%$ & $0.1 \%$ & $0.0 \%$ & $0.1 \%$ & $0.0 \%$ & 2.16529 \\
13 & $0.0 \%$ & $0.6 \%$ & $0.0 \%$ & $0.0 \%$ & $0.0 \%$ & $0.1 \%$ & $0.0 \%$ & 2.11052 \\
14 & $0.0 \%$ & $45.2 \%$ & $1.4 \%$ & $1.1 \%$ & $0.0 \%$ & $1.1 \%$ & $0.0 \%$ & 2.41551 \\
15 & $0.8 \%$ & $0.1 \%$ & $0.7 \%$ & $0.0 \%$ & $0.1 \%$ & $0.0 \%$ & $0.1 \%$ & 2.16479 \\
16 & $46.3 \%$ & $0.0 \%$ & $0.6 \%$ & $0.0 \%$ & $0.0 \%$ & $0.0 \%$ & $0.1 \%$ & 2.11045 \\
17 & $1.0 \%$ & $1.4 \%$ & $45.2 \%$ & $0.0 \%$ & $1.1 \%$ & $0.0 \%$ & $1.1 \%$ & 2.41585 \\
18 & $0.0 \%$ & $48.3 \%$ & $48.3 \%$ & $0.6 \%$ & $0.6 \%$ & $0.6 \%$ & $0.6 \%$ & 2.14662 \\
19 & $0.0 \%$ & $50.8 \%$ & $0.9 \%$ & $46.1 \%$ & $0.2 \%$ & $0.8 \%$ & $0.1 \%$ & 2.12112 \\
20 & $0.0 \%$ & $51.1 \%$ & $0.9 \%$ & $0.8 \%$ & $0.1 \%$ & $46.0 \%$ & $0.2 \%$ & 2.11637 \\
21 & $0.0 \%$ & $0.9 \%$ & $50.8 \%$ & $0.2 \%$ & $46.1 \%$ & $0.1 \%$ & $0.8 \%$ & 2.12172 \\
22 & $0.1 \%$ & $0.9 \%$ & $51.0 \%$ & $0.1 \%$ & $0.8 \%$ & $0.2 \%$ & $46.0 \%$ & 2.11714 \\
23 & $0.0 \%$ & $0.9 \%$ & $0.9 \%$ & $0.1 \%$ & $0.1 \%$ & $0.1 \%$ & $0.1 \%$ & 2.51213 \\
\hline \hline
\end{tabular}

S1.4.14 IRC Step 26

\begin{tabular}{ccl}
\hline \hline \multicolumn{3}{c}{ 1-center FNO } \\
\hline MO & $\lambda$ & Atoms,$\varepsilon=0.90000$ \\
\hline 1 & 0.99999 & $\mathrm{C}_{1}$ \\
2 & 0.99999 & $\mathrm{C}_{2}$ \\
3 & 0.99999 & $\mathrm{C}_{5}$ \\
4 & 0.99999 & $\mathrm{C}_{6}$ \\
5 & 0.99999 & $\mathrm{C}_{11}$ \\
6 & 0.99999 & $\mathrm{C}_{12}$ \\
\hline 2 -center FNO & analysis, $\varepsilon=0.90000$ \\
\hline MO & $\lambda$ & Atoms \\
\hline 7 & 0.97686 & $\mathrm{C}_{1}-\mathrm{C}_{2}$ \\
8 & 0.97677 & $\mathrm{C}_{1}-\mathrm{C}_{2}$ \\
9 & 0.97345 & $\mathrm{C}_{1}-\mathrm{H}_{3}$ \\
10 & 0.97661 & $\mathrm{C}_{1}-\mathrm{C}_{5}$ \\
11 & 0.97333 & $\mathrm{C}_{2}-\mathrm{H}_{4}$ \\
12 & 0.97333 & $\mathrm{C}_{2}-\mathrm{C}_{6}$ \\
13 & 0.96285 & $\mathrm{C}_{5}-\mathrm{H}_{7}$ \\
14 & 0.97544 & $\mathrm{C}_{5}-\mathrm{H}_{9}$ \\
15 & 0.96297 & $\mathrm{C}_{5}-\mathrm{C}_{11}$ \\
16 & 0.97541 & $\mathrm{C}_{6}-\mathrm{H}_{8}$ \\
17 & 0.97323 & $\mathrm{C}_{6}-\mathrm{H}_{10}$ \\
18 & 0.97117 & $\mathrm{C}_{6}-\mathrm{C}_{12}$ \\
19 & 0.97220 & $\mathrm{C}_{11}-\mathrm{C}_{12}$ \\
20 & 0.97119 & $\mathrm{C}_{11}-\mathrm{H}_{13}$ \\
21 & 0.97224 & $\mathrm{C}_{11}-\mathrm{H}_{15}$ \\
22 & 0.97119 & $\mathrm{C}_{12}-\mathrm{H}_{14}$ \\
23 & 0.97224 & $\mathrm{C}_{12}-\mathrm{H}_{16}$ \\
\hline \hline
\end{tabular}




\begin{tabular}{crrrrrrrrr}
\hline \multicolumn{2}{c}{ Localization and atoms expanded by each FNO } \\
\hline FNO & Loc $_{1}$ & $\mathrm{Loc}_{2}$ & $\mathrm{LOc}_{3}$ & $\mathrm{Loc}_{4}$ & $\mathrm{Loc}_{5}$ & $\mathrm{Loc}_{6}$ & $\mathrm{Loc}_{7}$ & $\mathrm{Loc}_{8}$ & Loc $_{9}$ \\
\hline 1 & $100.0 \%$ & $0.0 \%$ & $0.0 \%$ & $0.0 \%$ & $0.0 \%$ & $0.0 \%$ & $0.0 \%$ & $0.0 \%$ & $0.0 \%$ \\
2 & $0.0 \%$ & $100.0 \%$ & $0.0 \%$ & $0.0 \%$ & $0.0 \%$ & $0.0 \%$ & $0.0 \%$ & $0.0 \%$ & $0.0 \%$ \\
3 & $0.0 \%$ & $0.0 \%$ & $0.0 \%$ & $0.0 \%$ & $100.0 \%$ & $0.0 \%$ & $0.0 \%$ & $0.0 \%$ & $0.0 \%$ \\
4 & $0.0 \%$ & $0.0 \%$ & $0.0 \%$ & $0.0 \%$ & $0.0 \%$ & $100.0 \%$ & $0.0 \%$ & $0.0 \%$ & $0.0 \%$ \\
5 & $0.0 \%$ & $0.0 \%$ & $0.0 \%$ & $0.0 \%$ & $0.0 \%$ & $0.0 \%$ & $0.0 \%$ & $0.0 \%$ & $0.0 \%$ \\
6 & $-0.0 \%$ & $0.0 \%$ & $0.0 \%$ & $0.0 \%$ & $0.0 \%$ & $0.0 \%$ & $0.0 \%$ & $0.0 \%$ & $0.0 \%$ \\
7 & $49.0 \%$ & $49.0 \%$ & $0.4 \%$ & $0.4 \%$ & $0.5 \%$ & $0.5 \%$ & $0.0 \%$ & $0.0 \%$ & $0.1 \%$ \\
8 & $45.4 \%$ & $45.3 \%$ & $1.7 \%$ & $1.7 \%$ & $1.8 \%$ & $1.9 \%$ & $0.4 \%$ & $0.4 \%$ & $0.0 \%$ \\
9 & $52.8 \%$ & $0.8 \%$ & $45.2 \%$ & $0.1 \%$ & $0.7 \%$ & $0.1 \%$ & $0.0 \%$ & $0.0 \%$ & $0.2 \%$ \\
10 & $49.8 \%$ & $0.8 \%$ & $0.5 \%$ & $0.1 \%$ & $46.5 \%$ & $0.2 \%$ & $0.6 \%$ & $0.0 \%$ & $0.7 \%$ \\
11 & $0.8 \%$ & $52.8 \%$ & $0.1 \%$ & $45.2 \%$ & $0.1 \%$ & $0.7 \%$ & $0.0 \%$ & $0.0 \%$ & $0.0 \%$ \\
12 & $0.8 \%$ & $49.8 \%$ & $0.1 \%$ & $0.5 \%$ & $0.2 \%$ & $46.5 \%$ & $0.0 \%$ & $0.6 \%$ & $0.0 \%$ \\
13 & $1.3 \%$ & $0.4 \%$ & $0.0 \%$ & $0.0 \%$ & $49.3 \%$ & $0.2 \%$ & $46.3 \%$ & $0.4 \%$ & $0.9 \%$ \\
14 & $1.0 \%$ & $0.1 \%$ & $0.1 \%$ & $0.0 \%$ & $49.8 \%$ & $0.0 \%$ & $0.9 \%$ & $0.0 \%$ & $47.0 \%$ \\
15 & $1.3 \%$ & $0.4 \%$ & $0.0 \%$ & $0.0 \%$ & $47.1 \%$ & $0.2 \%$ & $0.9 \%$ & $0.0 \%$ & $0.8 \%$ \\
16 & $0.4 \%$ & $1.3 \%$ & $0.0 \%$ & $0.0 \%$ & $0.2 \%$ & $49.3 \%$ & $0.4 \%$ & $46.3 \%$ & $0.0 \%$ \\
17 & $0.1 \%$ & $1.0 \%$ & $0.0 \%$ & $0.1 \%$ & $0.0 \%$ & $49.7 \%$ & $0.0 \%$ & $0.9 \%$ & $0.0 \%$ \\
18 & $0.4 \%$ & $1.3 \%$ & $0.0 \%$ & $0.0 \%$ & $0.2 \%$ & $47.1 \%$ & $0.0 \%$ & $0.9 \%$ & $0.0 \%$ \\
19 & $0.1 \%$ & $0.1 \%$ & $0.0 \%$ & $0.0 \%$ & $0.6 \%$ & $0.6 \%$ & $0.0 \%$ & $0.0 \%$ & $0.1 \%$ \\
20 & $0.1 \%$ & $0.0 \%$ & $0.0 \%$ & $0.0 \%$ & $0.9 \%$ & $0.0 \%$ & $0.1 \%$ & $0.0 \%$ & $0.1 \%$ \\
21 & $0.2 \%$ & $0.0 \%$ & $0.0 \%$ & $0.0 \%$ & $0.9 \%$ & $0.0 \%$ & $0.1 \%$ & $0.0 \%$ & $0.1 \%$ \\
22 & $0.1 \%$ & $0.1 \%$ & $0.0 \%$ & $0.0 \%$ & $0.0 \%$ & $0.9 \%$ & $0.0 \%$ & $0.1 \%$ & $0.0 \%$ \\
23 & $0.0 \%$ & $0.2 \%$ & $0.0 \%$ & $0.0 \%$ & $0.0 \%$ & $0.9 \%$ & $0.0 \%$ & $0.1 \%$ & $0.0 \%$ \\
\hline \hline \hline
\end{tabular}

\begin{tabular}{crrrrrrrr}
\hline \hline \multicolumn{7}{c}{ Localization and atoms expanded by each FNO } \\
\hline FNO & Loc $_{10}$ & Loc $_{11}$ & Loc $_{12}$ & Loc $_{13}$ & Loc $_{14}$ & Loc $_{15}$ & Loc $_{16}$ & $n_{\text {eff }}$ \\
\hline 1 & $0.0 \%$ & $0.0 \%$ & $0.0 \%$ & $0.0 \%$ & $0.0 \%$ & $0.0 \%$ & $0.0 \%$ & 1.00002 \\
2 & $0.0 \%$ & $0.0 \%$ & $0.0 \%$ & $0.0 \%$ & $0.0 \%$ & $0.0 \%$ & $0.0 \%$ & 1.00002 \\
3 & $0.0 \%$ & $0.0 \%$ & $0.0 \%$ & $0.0 \%$ & $0.0 \%$ & $0.0 \%$ & $0.0 \%$ & 1.00002 \\
4 & $0.0 \%$ & $0.0 \%$ & $0.0 \%$ & $0.0 \%$ & $0.0 \%$ & $0.0 \%$ & $0.0 \%$ & 1.00002 \\
5 & $0.0 \%$ & $100.0 \%$ & $0.0 \%$ & $0.0 \%$ & $0.0 \%$ & $0.0 \%$ & $0.0 \%$ & 1.00001 \\
6 & $0.0 \%$ & $0.0 \%$ & $100.0 \%$ & $0.0 \%$ & $0.0 \%$ & $0.0 \%$ & $0.0 \%$ & 1.00001 \\
7 & $0.1 \%$ & $0.0 \%$ & $0.0 \%$ & $0.0 \%$ & $0.0 \%$ & $0.0 \%$ & $0.0 \%$ & 2.08476 \\
8 & $0.0 \%$ & $0.5 \%$ & $0.5 \%$ & $0.1 \%$ & $0.1 \%$ & $0.1 \%$ & $0.1 \%$ & 2.42436 \\
9 & $0.0 \%$ & $0.0 \%$ & $0.0 \%$ & $0.0 \%$ & $0.0 \%$ & $0.0 \%$ & $0.0 \%$ & 2.06835 \\
10 & $0.0 \%$ & $0.6 \%$ & $0.1 \%$ & $0.1 \%$ & $0.0 \%$ & $0.0 \%$ & $0.0 \%$ & 2.15221 \\
11 & $0.2 \%$ & $0.0 \%$ & $0.0 \%$ & $0.0 \%$ & $0.0 \%$ & $0.0 \%$ & $0.0 \%$ & 2.06867 \\
12 & $0.7 \%$ & $0.1 \%$ & $0.6 \%$ & $0.0 \%$ & $0.1 \%$ & $0.0 \%$ & $0.0 \%$ & 2.15244 \\
13 & $0.0 \%$ & $0.9 \%$ & $0.1 \%$ & $0.1 \%$ & $0.0 \%$ & $0.1 \%$ & $0.0 \%$ & 2.18689 \\
14 & $0.0 \%$ & $0.8 \%$ & $0.1 \%$ & $0.1 \%$ & $0.0 \%$ & $0.1 \%$ & $0.0 \%$ & 2.13436 \\
15 & $0.0 \%$ & $46.5 \%$ & $0.9 \%$ & $0.9 \%$ & $0.0 \%$ & $0.8 \%$ & $0.0 \%$ & 2.27793 \\
16 & $0.9 \%$ & $0.1 \%$ & $0.9 \%$ & $0.0 \%$ & $0.1 \%$ & $0.0 \%$ & $0.1 \%$ & 2.18688 \\
17 & $47.0 \%$ & $0.1 \%$ & $0.8 \%$ & $0.0 \%$ & $0.1 \%$ & $0.0 \%$ & $0.1 \%$ & 2.13456 \\
18 & $0.8 \%$ & $0.9 \%$ & $46.5 \%$ & $0.0 \%$ & $0.9 \%$ & $0.0 \%$ & $0.8 \%$ & 2.27842 \\
19 & $0.1 \%$ & $47.8 \%$ & $47.8 \%$ & $0.7 \%$ & $0.7 \%$ & $0.7 \%$ & $0.7 \%$ & 2.18925 \\
20 & $0.0 \%$ & $49.7 \%$ & $0.9 \%$ & $47.0 \%$ & $0.2 \%$ & $0.9 \%$ & $0.0 \%$ & 2.13849 \\
21 & $0.0 \%$ & $49.8 \%$ & $0.9 \%$ & $0.9 \%$ & $0.0 \%$ & $46.8 \%$ & $0.2 \%$ & 2.13661 \\
22 & $0.1 \%$ & $0.9 \%$ & $49.7 \%$ & $0.2 \%$ & $47.0 \%$ & $0.0 \%$ & $0.9 \%$ & 2.13807 \\
23 & $0.1 \%$ & $0.9 \%$ & $49.8 \%$ & $0.0 \%$ & $0.9 \%$ & $0.2 \%$ & $46.8 \%$ & 2.13741 \\
\hline \hline
\end{tabular}


S1.4.15 IRC Step 28

\begin{tabular}{|c|c|c|}
\hline \multicolumn{3}{|c|}{ 1-center FNO analysis, $\varepsilon=0.90000$} \\
\hline $\mathrm{MO}$ & $\lambda$ & Atoms \\
\hline 1 & 0.99999 & $\mathrm{C}_{1}$ \\
\hline 2 & 0.99999 & $\mathrm{C}_{2}$ \\
\hline 3 & 0.99999 & $\mathrm{C}_{5}$ \\
\hline 4 & 0.99999 & $\mathrm{C}_{6}$ \\
\hline 5 & 0.99999 & $\mathrm{C}_{11}$ \\
\hline 6 & 0.99999 & $\mathrm{C}_{12}$ \\
\hline \multicolumn{3}{|c|}{ 2-center FNO analysis, $\varepsilon=0.90000$} \\
\hline MO & $\lambda$ & Atoms \\
\hline 7 & 0.97950 & $\mathrm{C}_{1}-\mathrm{C}_{2}$ \\
\hline 8 & 0.91012 & $\mathrm{C}_{1}-\mathrm{C}_{2}$ \\
\hline 9 & 0.97998 & $\mathrm{C}_{1}-\mathrm{H}_{3}$ \\
\hline 10 & 0.96117 & $\mathrm{C}_{1}-\mathrm{C}_{5}$ \\
\hline 11 & 0.97988 & $\mathrm{C}_{2}-\mathrm{H}_{4}$ \\
\hline 12 & 0.96110 & $\mathrm{C}_{2}-\mathrm{C}_{6}$ \\
\hline 13 & 0.95460 & $\mathrm{C}_{5}-\mathrm{H}_{7}$ \\
\hline 14 & 0.96391 & $\mathrm{C}_{5}-\mathrm{H}_{9}$ \\
\hline 15 & 0.94434 & $\mathrm{C}_{5}-\mathrm{C}_{11}$ \\
\hline 16 & 0.95464 & $\mathrm{C}_{6}-\mathrm{H}_{8}$ \\
\hline 17 & 0.96387 & $\mathrm{C}_{6}-\mathrm{H}_{10}$ \\
\hline 18 & 0.94448 & $\mathrm{C}_{6}-\mathrm{C}_{12}$ \\
\hline 19 & 0.95145 & $\mathrm{C}_{11}-\mathrm{C}_{12}$ \\
\hline 20 & 0.96389 & $\mathrm{C}_{11}-\mathrm{H}_{13}$ \\
\hline 21 & 0.96251 & $\mathrm{C}_{11}-\mathrm{H}_{15}$ \\
\hline 22 & 0.96400 & $\mathrm{C}_{12}-\mathrm{H}_{14}$ \\
\hline 23 & 0.96246 & $\mathrm{C}_{12}-\mathrm{H}_{16}$ \\
\hline
\end{tabular}

\begin{tabular}{crrrrrrrrr}
\hline \hline \multicolumn{7}{l}{ Localization and atoms expanded by each FNO } \\
\hline FNO & Loc $_{1}$ & Loc $_{2}$ & Loc $_{3}$ & Loc $_{4}$ & Loc $_{5}$ & Loc $_{6}$ & Loc $_{7}$ & Loc $_{8}$ & Loc $_{9}$ \\
\hline 1 & $100.0 \%$ & $0.0 \%$ & $0.0 \%$ & $0.0 \%$ & $0.0 \%$ & $0.0 \%$ & $0.0 \%$ & $0.0 \%$ & $0.0 \%$ \\
2 & $0.0 \%$ & $100.0 \%$ & $0.0 \%$ & $0.0 \%$ & $0.0 \%$ & $0.0 \%$ & $0.0 \%$ & $0.0 \%$ & $0.0 \%$ \\
3 & $0.0 \%$ & $0.0 \%$ & $0.0 \%$ & $0.0 \%$ & $100.0 \%$ & $0.0 \%$ & $0.0 \%$ & $0.0 \%$ & $0.0 \%$ \\
4 & $0.0 \%$ & $0.0 \%$ & $0.0 \%$ & $0.0 \%$ & $0.0 \%$ & $100.0 \%$ & $0.0 \%$ & $0.0 \%$ & $0.0 \%$ \\
5 & $0.0 \%$ & $0.0 \%$ & $0.0 \%$ & $0.0 \%$ & $0.0 \%$ & $0.0 \%$ & $0.0 \%$ & $0.0 \%$ & $0.0 \%$ \\
6 & $-0.0 \%$ & $0.0 \%$ & $0.0 \%$ & $0.0 \%$ & $0.0 \%$ & $0.0 \%$ & $0.0 \%$ & $0.0 \%$ & $0.0 \%$ \\
7 & $49.0 \%$ & $49.0 \%$ & $0.4 \%$ & $0.4 \%$ & $0.5 \%$ & $0.5 \%$ & $0.0 \%$ & $0.0 \%$ & $0.1 \%$ \\
8 & $45.5 \%$ & $45.5 \%$ & $1.7 \%$ & $1.7 \%$ & $1.7 \%$ & $1.7 \%$ & $0.4 \%$ & $0.4 \%$ & $0.0 \%$ \\
9 & $52.7 \%$ & $0.8 \%$ & $45.3 \%$ & $0.1 \%$ & $0.7 \%$ & $0.1 \%$ & $0.0 \%$ & $0.0 \%$ & $0.2 \%$ \\
10 & $50.2 \%$ & $0.8 \%$ & $0.5 \%$ & $0.1 \%$ & $45.9 \%$ & $0.2 \%$ & $0.7 \%$ & $0.0 \%$ & $0.7 \%$ \\
11 & $0.9 \%$ & $52.7 \%$ & $0.1 \%$ & $45.3 \%$ & $0.1 \%$ & $0.7 \%$ & $0.0 \%$ & $0.0 \%$ & $0.0 \%$ \\
12 & $0.8 \%$ & $50.2 \%$ & $0.1 \%$ & $0.5 \%$ & $0.2 \%$ & $45.9 \%$ & $0.0 \%$ & $0.7 \%$ & $0.0 \%$ \\
13 & $1.4 \%$ & $0.4 \%$ & $0.0 \%$ & $0.0 \%$ & $48.7 \%$ & $0.1 \%$ & $46.7 \%$ & $0.2 \%$ & $1.0 \%$ \\
14 & $1.0 \%$ & $0.1 \%$ & $0.2 \%$ & $0.0 \%$ & $49.1 \%$ & $0.0 \%$ & $0.9 \%$ & $0.0 \%$ & $47.3 \%$ \\
15 & $1.1 \%$ & $0.3 \%$ & $0.1 \%$ & $0.0 \%$ & $47.5 \%$ & $0.2 \%$ & $0.8 \%$ & $0.0 \%$ & $0.7 \%$ \\
16 & $0.4 \%$ & $1.4 \%$ & $0.0 \%$ & $0.0 \%$ & $0.1 \%$ & $48.7 \%$ & $0.2 \%$ & $46.7 \%$ & $0.0 \%$ \\
17 & $0.1 \%$ & $1.0 \%$ & $0.0 \%$ & $0.2 \%$ & $0.0 \%$ & $49.1 \%$ & $0.0 \%$ & $0.9 \%$ & $0.0 \%$ \\
18 & $0.3 \%$ & $1.1 \%$ & $0.0 \%$ & $0.1 \%$ & $0.2 \%$ & $47.5 \%$ & $0.0 \%$ & $0.8 \%$ & $0.0 \%$ \\
19 & $0.1 \%$ & $0.1 \%$ & $0.0 \%$ & $0.0 \%$ & $0.7 \%$ & $0.7 \%$ & $0.0 \%$ & $0.0 \%$ & $0.1 \%$ \\
20 & $0.2 \%$ & $0.0 \%$ & $0.0 \%$ & $0.0 \%$ & $1.0 \%$ & $0.0 \%$ & $0.2 \%$ & $0.0 \%$ & $0.1 \%$ \\
21 & $0.2 \%$ & $0.0 \%$ & $0.0 \%$ & $0.0 \%$ & $1.0 \%$ & $0.0 \%$ & $0.1 \%$ & $0.0 \%$ & $0.1 \%$ \\
22 & $0.1 \%$ & $0.1 \%$ & $0.0 \%$ & $0.0 \%$ & $0.0 \%$ & $1.0 \%$ & $0.0 \%$ & $0.2 \%$ & $0.0 \%$ \\
23 & $0.0 \%$ & $0.2 \%$ & $0.0 \%$ & $0.0 \%$ & $0.0 \%$ & $1.0 \%$ & $0.0 \%$ & $0.1 \%$ & $0.0 \%$ \\
\hline \hline
\end{tabular}




\begin{tabular}{crrrrrrrr}
\hline \multicolumn{2}{c}{ Localization and atoms expanded by each FNO } \\
\hline FNO & Loc $_{10}$ & Loc $_{11}$ & Loc $_{12}$ & Loc $_{13}$ & Loc $_{14}$ & Loc $_{15}$ & Loc $_{16}$ & $n_{\text {eff }}$ \\
\hline 1 & $0.0 \%$ & $0.0 \%$ & $0.0 \%$ & $0.0 \%$ & $0.0 \%$ & $0.0 \%$ & $0.0 \%$ & 1.00002 \\
2 & $0.0 \%$ & $0.0 \%$ & $0.0 \%$ & $0.0 \%$ & $0.0 \%$ & $0.0 \%$ & $0.0 \%$ & 1.00002 \\
3 & $0.0 \%$ & $0.0 \%$ & $0.0 \%$ & $0.0 \%$ & $0.0 \%$ & $0.0 \%$ & $0.0 \%$ & 1.00002 \\
4 & $0.0 \%$ & $0.0 \%$ & $0.0 \%$ & $0.0 \%$ & $0.0 \%$ & $0.0 \%$ & $0.0 \%$ & 1.00002 \\
5 & $0.0 \%$ & $100.0 \%$ & $0.0 \%$ & $0.0 \%$ & $0.0 \%$ & $0.0 \%$ & $0.0 \%$ & 1.00002 \\
6 & $0.0 \%$ & $0.0 \%$ & $100.0 \%$ & $0.0 \%$ & $0.0 \%$ & $0.0 \%$ & $0.0 \%$ & 1.00002 \\
7 & $0.1 \%$ & $0.1 \%$ & $0.1 \%$ & $0.0 \%$ & $0.0 \%$ & $0.0 \%$ & $0.0 \%$ & 2.08427 \\
8 & $0.0 \%$ & $0.4 \%$ & $0.4 \%$ & $0.1 \%$ & $0.1 \%$ & $0.1 \%$ & $0.1 \%$ & 2.40748 \\
9 & $0.0 \%$ & $0.0 \%$ & $0.0 \%$ & $0.0 \%$ & $0.0 \%$ & $0.0 \%$ & $0.0 \%$ & 2.07010 \\
10 & $0.0 \%$ & $0.7 \%$ & $0.1 \%$ & $0.1 \%$ & $0.0 \%$ & $0.0 \%$ & $0.0 \%$ & 2.15944 \\
11 & $0.2 \%$ & $0.0 \%$ & $0.0 \%$ & $0.0 \%$ & $0.0 \%$ & $0.0 \%$ & $0.0 \%$ & 2.07055 \\
12 & $0.7 \%$ & $0.1 \%$ & $0.7 \%$ & $0.0 \%$ & $0.1 \%$ & $0.0 \%$ & $0.0 \%$ & 2.15977 \\
13 & $0.0 \%$ & $1.0 \%$ & $0.1 \%$ & $0.2 \%$ & $0.0 \%$ & $0.1 \%$ & $0.0 \%$ & 2.19186 \\
14 & $0.0 \%$ & $1.0 \%$ & $0.1 \%$ & $0.1 \%$ & $0.0 \%$ & $0.2 \%$ & $0.0 \%$ & 2.15042 \\
15 & $0.0 \%$ & $46.9 \%$ & $0.7 \%$ & $0.8 \%$ & $0.0 \%$ & $0.8 \%$ & $0.0 \%$ & 2.24050 \\
16 & $1.0 \%$ & $0.1 \%$ & $1.0 \%$ & $0.0 \%$ & $0.1 \%$ & $0.0 \%$ & $0.1 \%$ & 2.19168 \\
17 & $47.3 \%$ & $0.1 \%$ & $1.0 \%$ & $0.0 \%$ & $0.1 \%$ & $0.0 \%$ & $0.2 \%$ & 2.15060 \\
18 & $0.7 \%$ & $0.7 \%$ & $46.9 \%$ & $0.0 \%$ & $0.8 \%$ & $0.0 \%$ & $0.8 \%$ & 2.23983 \\
19 & $0.1 \%$ & $47.6 \%$ & $47.6 \%$ & $0.7 \%$ & $0.7 \%$ & $0.7 \%$ & $0.7 \%$ & 2.20778 \\
20 & $0.0 \%$ & $48.9 \%$ & $0.9 \%$ & $47.5 \%$ & $0.2 \%$ & $0.9 \%$ & $0.0 \%$ & 2.15091 \\
21 & $0.0 \%$ & $48.9 \%$ & $0.9 \%$ & $0.9 \%$ & $0.0 \%$ & $47.3 \%$ & $0.3 \%$ & 2.15688 \\
22 & $0.1 \%$ & $0.9 \%$ & $48.9 \%$ & $0.2 \%$ & $47.5 \%$ & $0.0 \%$ & $0.9 \%$ & 2.15041 \\
23 & $0.1 \%$ & $0.9 \%$ & $48.9 \%$ & $0.0 \%$ & $0.9 \%$ & $0.3 \%$ & $47.3 \%$ & 2.15713 \\
\hline \hline
\end{tabular}




\section{S1.5 The $\mathbf{F}^{-}+\mathrm{CH}_{3} \mathbf{F} \rightarrow \mathrm{FCH}_{3}+\mathbf{F}^{-}$reaction}

Due to the symmetry of the fluorine self-substitution reaction only the forward reaction profile has been analyzed. The geometry of the products is equivalent to that of the reactants. The evolution of the degree of localization and the effective number of centers along the IRC is shown in the following subsections.

\section{S1.5.1 IRC Step 1}

\begin{tabular}{|c|c|c|c|c|c|c|c|c|}
\hline \multicolumn{4}{|c|}{ 1-center FNO analysis, $\varepsilon=0.85000$} & & & & & \\
\hline $\mathrm{MO}$ & $\lambda$ & \multicolumn{2}{|l|}{ Atoms } & & & & & \\
\hline 1 & 1.00177 & \multicolumn{2}{|l|}{$\mathrm{C}_{1}$} & & & & & \\
\hline 2 & 1.00022 & \multicolumn{2}{|l|}{$\mathrm{F}_{2}$} & & & & & \\
\hline 3 & 0.99852 & \multicolumn{2}{|l|}{$\mathrm{F}_{2}$} & & & & & \\
\hline 4 & 0.98070 & \multicolumn{2}{|l|}{$\mathrm{F}_{2}$} & & & & & \\
\hline 5 & 0.98067 & \multicolumn{2}{|l|}{$\mathrm{F}_{2}$} & & & & & \\
\hline 6 & 1.00004 & \multicolumn{2}{|l|}{$\mathrm{F}_{6}$} & & & & & \\
\hline 7 & 0.99977 & \multicolumn{2}{|l|}{$\mathrm{F}_{6}$} & & & & & \\
\hline 8 & 0.99260 & \multicolumn{2}{|l|}{$\mathrm{F}_{6}$} & & & & & \\
\hline 9 & 0.99259 & \multicolumn{2}{|l|}{$\mathrm{F}_{6}$} & & & & & \\
\hline 10 & 0.97394 & \multicolumn{2}{|l|}{$\mathrm{F}_{6}$} & & & & & \\
\hline \multicolumn{9}{|c|}{ 2-center FNO analysis, $\varepsilon=0.85000$} \\
\hline $\mathrm{MO}$ & $\lambda$ & \multicolumn{2}{|l|}{ Atoms } & & & & & \\
\hline 11 & 0.99226 & \multicolumn{2}{|l|}{$\mathrm{C}_{1}-\mathrm{F}_{2}$} & & & & & \\
\hline 12 & 0.96012 & \multicolumn{2}{|l|}{$\mathrm{C}_{1}-\mathrm{H}_{3}$} & & & & & \\
\hline 13 & 0.96001 & \multicolumn{2}{|l|}{$\mathrm{C}_{1}-\mathrm{H}_{4}$} & & & & & \\
\hline 14 & 0.96025 & \multicolumn{2}{|l|}{$\mathrm{C}_{1}-\mathrm{H}_{5}$} & & & & & \\
\hline \multicolumn{9}{|c|}{ Localization and atoms expanded by each FNO } \\
\hline FNO & $\mathrm{Loc}_{1}$ & $\mathrm{Loc}_{2}$ & $\mathrm{Loc}_{3}$ & $\mathrm{LOC}_{4}$ & $\mathrm{LOc}_{5}$ & $\mathrm{Loc}_{6}$ & $\mathrm{LOC}_{7}$ & $n_{\mathrm{eff}}$ \\
\hline 1 & $100.2 \%$ & $0.0 \%$ & $0.0 \%$ & $0.0 \%$ & $0.0 \%$ & $0.0 \%$ & 0.99647 & \\
\hline 2 & $0.0 \%$ & $100.0 \%$ & $0.0 \%$ & $0.0 \%$ & $0.0 \%$ & $0.0 \%$ & 0.99957 & \\
\hline 3 & $0.1 \%$ & $99.9 \%$ & $0.0 \%$ & $0.0 \%$ & $0.0 \%$ & $0.0 \%$ & 1.00296 & \\
\hline 4 & $1.4 \%$ & $98.1 \%$ & $0.0 \%$ & $0.2 \%$ & $0.3 \%$ & $0.0 \%$ & 1.03953 & \\
\hline 5 & $1.4 \%$ & $98.1 \%$ & $0.3 \%$ & $0.1 \%$ & $0.1 \%$ & $0.0 \%$ & 1.03960 & \\
\hline 6 & $0.0 \%$ & $0.0 \%$ & $0.0 \%$ & $0.0 \%$ & $0.0 \%$ & $100.0 \%$ & 0.99992 & \\
\hline 7 & $0.0 \%$ & $0.0 \%$ & $0.0 \%$ & $0.0 \%$ & $0.0 \%$ & $100.0 \%$ & 1.00046 & \\
\hline 8 & $0.1 \%$ & $0.0 \%$ & $0.3 \%$ & $0.2 \%$ & $0.1 \%$ & $99.3 \%$ & 1.01495 & \\
\hline 9 & $0.1 \%$ & $0.0 \%$ & $0.1 \%$ & $0.2 \%$ & $0.3 \%$ & $99.3 \%$ & 1.01498 & \\
\hline 10 & $1.2 \%$ & $0.2 \%$ & $0.4 \%$ & $0.4 \%$ & $0.4 \%$ & $97.4 \%$ & 1.05401 & \\
\hline 11 & $18.9 \%$ & $80.3 \%$ & $0.2 \%$ & $0.2 \%$ & $0.2 \%$ & $0.1 \%$ & 1.46803 & \\
\hline 12 & $51.3 \%$ & $1.9 \%$ & $44.8 \%$ & $0.7 \%$ & $0.7 \%$ & $0.6 \%$ & 2.15730 & \\
\hline 13 & $51.3 \%$ & $1.9 \%$ & $0.7 \%$ & $44.7 \%$ & $0.7 \%$ & $0.6 \%$ & 2.15772 & \\
\hline 14 & $51.3 \%$ & $1.9 \%$ & $0.7 \%$ & $0.7 \%$ & $44.8 \%$ & $0.6 \%$ & 2.15670 & \\
\hline
\end{tabular}

\section{S1.5.2 IRC Step 2}

\begin{tabular}{ccc}
\hline \hline \multicolumn{3}{c}{ 1-center FNO analysis, $\varepsilon=0.85000$} \\
\hline MO & $\lambda$ & Atoms \\
\hline 1 & 1.00177 & $\mathrm{C}_{1}$ \\
2 & 1.00022 & $\mathrm{~F}_{2}$ \\
3 & 0.99853 & $\mathrm{~F}_{2}$ \\
4 & 0.98073 & $\mathrm{~F}_{2}$ \\
5 & 0.98070 & $\mathrm{~F}_{2}$ \\
6 & 1.00004 & $\mathrm{~F}_{6}$ \\
7 & 0.99974 & $\mathrm{~F}_{6}$ \\
8 & 0.99230 & $\mathrm{~F}_{6}$ \\
9 & 0.99229 & $\mathrm{~F}_{6}$ \\
10 & 0.97310 & $\mathrm{~F}_{6}$ \\
\hline 2 -center $\mathrm{FNO}$ analysis, $\varepsilon=0.85000$ \\
\hline MO & $\lambda$ & Atoms \\
\hline 11 & 0.99224 & $\mathrm{C}_{1}-\mathrm{F}_{2}$ \\
12 & 0.95967 & $\mathrm{C}_{1}-\mathrm{H}_{3}$ \\
13 & 0.96001 & $\mathrm{C}_{1}-\mathrm{H}_{4}$ \\
14 & 0.96022 & $\mathrm{C}_{1}-\mathrm{H}_{5}$ \\
\hline \hline
\end{tabular}




\begin{tabular}{crrrrrrrr}
\hline \hline \multicolumn{2}{l}{ Localization and atoms expanded by each FNO } \\
\hline FNO & Loc $_{1}$ & \multicolumn{1}{l}{ Loc $_{2}$} & Loc $_{3}$ & Loc $_{4}$ & Loc $_{5}$ & Loc $_{6}$ & Loc $_{7}$ & $n_{\text {eff }}$ \\
\hline 1 & $100.2 \%$ & $0.0 \%$ & $0.0 \%$ & $0.0 \%$ & $0.0 \%$ & $0.0 \%$ & 0.99647 & \\
2 & $0.0 \%$ & $100.0 \%$ & $0.0 \%$ & $0.0 \%$ & $0.0 \%$ & $0.0 \%$ & 0.99957 & \\
3 & $0.1 \%$ & $99.9 \%$ & $0.0 \%$ & $0.0 \%$ & $0.0 \%$ & $0.0 \%$ & 1.00295 & \\
4 & $1.4 \%$ & $98.1 \%$ & $0.0 \%$ & $0.2 \%$ & $0.3 \%$ & $0.0 \%$ & 1.03946 & \\
5 & $1.4 \%$ & $98.1 \%$ & $0.3 \%$ & $0.1 \%$ & $0.1 \%$ & $0.0 \%$ & 1.03953 & \\
6 & $0.0 \%$ & $0.0 \%$ & $0.0 \%$ & $0.0 \%$ & $0.0 \%$ & $100.0 \%$ & 0.99992 & \\
7 & $0.0 \%$ & $0.0 \%$ & $0.0 \%$ & $0.0 \%$ & $0.0 \%$ & $100.0 \%$ & 1.00051 & \\
8 & $0.1 \%$ & $0.0 \%$ & $0.2 \%$ & $0.1 \%$ & $0.3 \%$ & $99.2 \%$ & 1.01556 & \\
9 & $0.1 \%$ & $0.0 \%$ & $0.2 \%$ & $0.3 \%$ & $0.1 \%$ & $99.2 \%$ & 1.01557 & \\
10 & $1.3 \%$ & $0.3 \%$ & $0.4 \%$ & $0.4 \%$ & $0.4 \%$ & $97.3 \%$ & 1.05581 & \\
11 & $18.9 \%$ & $80.4 \%$ & $0.2 \%$ & $0.2 \%$ & $0.2 \%$ & $0.1 \%$ & 1.46727 & \\
12 & $51.2 \%$ & $1.9 \%$ & $44.7 \%$ & $0.7 \%$ & $0.7 \%$ & $0.7 \%$ & 2.15936 & \\
13 & $51.2 \%$ & $1.9 \%$ & $0.7 \%$ & $44.8 \%$ & $0.7 \%$ & $0.7 \%$ & 2.15783 & \\
14 & $51.3 \%$ & $1.9 \%$ & $0.7 \%$ & $0.7 \%$ & $44.8 \%$ & $0.7 \%$ & 2.15682 & \\
\hline \hline
\end{tabular}

\section{S1.5.3 IRC Step 3}

\begin{tabular}{|c|c|c|}
\hline \multicolumn{3}{|c|}{1 1-center FNO analysis, $\varepsilon=0.85000$} \\
\hline $\mathrm{MO}$ & $\lambda$ & Atoms \\
\hline 1 & 1.00177 & $\mathrm{C}_{1}$ \\
\hline 2 & 1.00022 & $\mathrm{~F}_{2}$ \\
\hline 3 & 0.99853 & $\mathrm{~F}_{2}$ \\
\hline 4 & 0.98074 & $\mathrm{~F}_{2}$ \\
\hline 5 & 0.98072 & $\mathrm{~F}_{2}$ \\
\hline 6 & 1.00004 & $\mathrm{~F}_{6}$ \\
\hline 7 & 0.99972 & $\mathrm{~F}_{6}$ \\
\hline 8 & 0.99199 & $\mathrm{~F}_{6}$ \\
\hline 9 & 0.99198 & $\mathrm{~F}_{6}$ \\
\hline 10 & 0.97204 & $\mathrm{~F}_{6}$ \\
\hline \multicolumn{3}{|c|}{2 -center FNO analysis, $\varepsilon=0.85000$} \\
\hline MO & $\lambda$ & Atoms \\
\hline 11 & 0.99234 & $\mathrm{C}_{1}-\mathrm{F}_{2}$ \\
\hline 12 & 0.95991 & $\mathrm{C}_{1}-\mathrm{H}_{3}$ \\
\hline 13 & 0.95968 & $\mathrm{C}_{1}-\mathrm{H}_{4}$ \\
\hline 14 & 0.95971 & $\mathrm{C}_{1}-\mathrm{H}_{5}$ \\
\hline
\end{tabular}

\begin{tabular}{crrrrrrrr}
\hline \multicolumn{2}{l}{ Localization and atoms expanded by each FNO } \\
\hline FNO & Loc $_{1}$ & Loc $_{2}$ & Loc $_{3}$ & Loc $_{4}$ & Loc $_{5}$ & Loc $_{6}$ & Loc $_{7}$ & $n_{\text {eff }}$ \\
\hline 1 & $100.2 \%$ & $0.0 \%$ & $0.0 \%$ & $0.0 \%$ & $0.0 \%$ & $0.0 \%$ & 0.99646 & \\
2 & $0.0 \%$ & $100.0 \%$ & $0.0 \%$ & $0.0 \%$ & $0.0 \%$ & $0.0 \%$ & 0.99957 & \\
3 & $0.1 \%$ & $99.9 \%$ & $0.0 \%$ & $0.0 \%$ & $0.0 \%$ & $0.0 \%$ & 1.00295 & \\
4 & $1.4 \%$ & $98.1 \%$ & $0.3 \%$ & $0.2 \%$ & $0.0 \%$ & $0.0 \%$ & 1.03945 & \\
5 & $1.4 \%$ & $98.1 \%$ & $0.0 \%$ & $0.2 \%$ & $0.3 \%$ & $0.0 \%$ & 1.03948 & \\
6 & $0.0 \%$ & $0.0 \%$ & $0.0 \%$ & $0.0 \%$ & $0.0 \%$ & $100.0 \%$ & 0.99991 & \\
7 & $0.0 \%$ & $0.0 \%$ & $0.0 \%$ & $0.0 \%$ & $0.0 \%$ & $100.0 \%$ & 1.00057 & \\
8 & $0.2 \%$ & $0.0 \%$ & $0.1 \%$ & $0.2 \%$ & $0.3 \%$ & $99.2 \%$ & 1.01619 & \\
9 & $0.2 \%$ & $0.0 \%$ & $0.3 \%$ & $0.2 \%$ & $0.1 \%$ & $99.2 \%$ & 1.01621 & \\
10 & $1.3 \%$ & $0.3 \%$ & $0.4 \%$ & $0.4 \%$ & $0.4 \%$ & $97.2 \%$ & 1.05809 & \\
11 & $18.8 \%$ & $80.4 \%$ & $0.2 \%$ & $0.2 \%$ & $0.2 \%$ & $0.1 \%$ & 1.46645 & \\
12 & $51.2 \%$ & $1.9 \%$ & $44.7 \%$ & $0.7 \%$ & $0.7 \%$ & $0.7 \%$ & 2.15820 & \\
13 & $51.2 \%$ & $1.9 \%$ & $0.7 \%$ & $44.7 \%$ & $0.7 \%$ & $0.7 \%$ & 2.15936 & \\
14 & $51.2 \%$ & $1.9 \%$ & $0.7 \%$ & $0.7 \%$ & $44.8 \%$ & $0.7 \%$ & 2.15935 & \\
\hline \hline
\end{tabular}




\section{S1.5.4 IRC Step 4}

\begin{tabular}{|c|c|c|}
\hline \multicolumn{3}{|c|}{ 1-center FNO analysis, $\varepsilon=0.85000$} \\
\hline $\mathrm{MO}$ & $\lambda$ & Atoms \\
\hline 1 & 1.00177 & $\mathrm{C}_{1}$ \\
\hline 2 & 1.00022 & $\mathrm{~F}_{2}$ \\
\hline 3 & 0.99853 & $\mathrm{~F}_{2}$ \\
\hline 4 & 0.98074 & $\mathrm{~F}_{2}$ \\
\hline 5 & 0.98072 & $\mathrm{~F}_{2}$ \\
\hline 6 & 1.00004 & $\mathrm{~F}_{6}$ \\
\hline 7 & 0.99969 & $\mathrm{~F}_{6}$ \\
\hline 8 & 0.99167 & $\mathrm{~F}_{6}$ \\
\hline 9 & 0.99167 & $\mathrm{~F}_{6}$ \\
\hline 10 & 0.97110 & $\mathrm{~F}_{6}$ \\
\hline \multicolumn{3}{|c|}{ 2-center FNO analysis, $\varepsilon=0.85000$} \\
\hline $\mathrm{MO}$ & $\lambda$ & Atoms \\
\hline 11 & 0.99228 & $\mathrm{C}_{1}-\mathrm{F}_{2}$ \\
\hline 12 & 0.95963 & $\mathrm{C}_{1}-\mathrm{H}_{3}$ \\
\hline 13 & 0.95950 & $\mathrm{C}_{1}-\mathrm{H}_{4}$ \\
\hline 14 & 0.95954 & $\mathrm{C}_{1}-\mathrm{H}_{5}$ \\
\hline
\end{tabular}

\begin{tabular}{crrrrrrrr}
\hline \multicolumn{7}{l}{ Localization and atoms expanded by each FNO } & & \\
\hline FNO & Loc $_{1}$ & Loc $_{2}$ & Loc $_{3}$ & Loc $_{4}$ & Loc $_{5}$ & Loc $_{6}$ & Loc $_{7}$ & $n_{\text {eff }}$ \\
\hline 1 & $100.2 \%$ & $0.0 \%$ & $0.0 \%$ & $0.0 \%$ & $0.0 \%$ & $0.0 \%$ & 0.99646 & \\
2 & $0.0 \%$ & $100.0 \%$ & $0.0 \%$ & $0.0 \%$ & $0.0 \%$ & $0.0 \%$ & 0.99957 & \\
3 & $0.1 \%$ & $99.9 \%$ & $0.0 \%$ & $0.0 \%$ & $0.0 \%$ & $0.0 \%$ & 1.00295 & \\
4 & $1.4 \%$ & $98.1 \%$ & $0.0 \%$ & $0.3 \%$ & $0.2 \%$ & $0.0 \%$ & 1.03945 & \\
5 & $1.4 \%$ & $98.1 \%$ & $0.3 \%$ & $0.1 \%$ & $0.1 \%$ & $0.0 \%$ & 1.03949 & \\
6 & $0.0 \%$ & $0.0 \%$ & $0.0 \%$ & $0.0 \%$ & $0.0 \%$ & $100.0 \%$ & 0.99991 & \\
7 & $0.0 \%$ & $0.0 \%$ & $0.0 \%$ & $0.0 \%$ & $0.0 \%$ & $100.0 \%$ & 1.00063 & \\
8 & $0.2 \%$ & $0.0 \%$ & $0.1 \%$ & $0.3 \%$ & $0.3 \%$ & $99.2 \%$ & 1.01684 & \\
9 & $0.2 \%$ & $0.0 \%$ & $0.3 \%$ & $0.2 \%$ & $0.1 \%$ & $99.2 \%$ & 1.01685 & \\
10 & $1.4 \%$ & $0.3 \%$ & $0.4 \%$ & $0.4 \%$ & $0.4 \%$ & $97.1 \%$ & 1.06013 & \\
11 & $18.8 \%$ & $80.4 \%$ & $0.2 \%$ & $0.2 \%$ & $0.2 \%$ & $0.1 \%$ & 1.46578 & \\
12 & $51.2 \%$ & $1.9 \%$ & $44.8 \%$ & $0.7 \%$ & $0.7 \%$ & $0.7 \%$ & 2.15963 & \\
13 & $51.2 \%$ & $1.9 \%$ & $0.7 \%$ & $44.8 \%$ & $0.7 \%$ & $0.7 \%$ & 2.16020 & \\
14 & $51.2 \%$ & $1.9 \%$ & $0.7 \%$ & $0.7 \%$ & $44.8 \%$ & $0.7 \%$ & 2.16002 & \\
\hline \hline
\end{tabular}

\section{S1.5.5 IRC Step 5}

\begin{tabular}{ccc}
\hline \hline \multicolumn{3}{c}{ 1-center FNO analysis, $\varepsilon=0.85000$} \\
\hline $\mathrm{MO}$ & $\lambda$ & Atoms \\
\hline 1 & 1.00178 & $\mathrm{C}_{1}$ \\
2 & 1.00022 & $\mathrm{~F}_{2}$ \\
3 & 0.99852 & $\mathrm{~F}_{2}$ \\
4 & 0.98072 & $\mathrm{~F}_{2}$ \\
5 & 0.98072 & $\mathrm{~F}_{2}$ \\
6 & 1.00005 & $\mathrm{~F}_{6}$ \\
7 & 0.99965 & $\mathrm{~F}_{6}$ \\
8 & 0.99134 & $\mathrm{~F}_{6}$ \\
9 & 0.99133 & $\mathrm{~F}_{6}$ \\
10 & 0.96995 & $\mathrm{~F}_{6}$ \\
\hline 2 -center FNO analysis, $\varepsilon=0.85000$ \\
\hline MO & $\lambda$ & Atoms \\
\hline 11 & 0.99211 & $\mathrm{C}_{1}-\mathrm{F}_{2}$ \\
12 & 0.95928 & $\mathrm{C}_{1}-\mathrm{H}_{3}$ \\
13 & 0.95926 & $\mathrm{C}_{1}-\mathrm{H}_{4}$ \\
14 & 0.95912 & $\mathrm{C}_{1}-\mathrm{H}_{5}$ \\
\hline \hline
\end{tabular}




\begin{tabular}{crrrrrrrr}
\hline \hline \multicolumn{2}{l}{ Localization and atoms expanded by each FNO } \\
\hline FNO & Loc $_{1}$ & \multicolumn{1}{l}{ Loc $_{2}$} & Loc $_{3}$ & Loc $_{4}$ & Loc $_{5}$ & Loc $_{6}$ & Loc $_{7}$ & $n_{\text {eff }}$ \\
\hline 1 & $100.2 \%$ & $0.0 \%$ & $0.0 \%$ & $0.0 \%$ & $0.0 \%$ & $0.0 \%$ & 0.99646 & \\
2 & $0.0 \%$ & $100.0 \%$ & $0.0 \%$ & $0.0 \%$ & $0.0 \%$ & $0.0 \%$ & 0.99957 & \\
3 & $0.1 \%$ & $99.9 \%$ & $0.0 \%$ & $0.0 \%$ & $0.0 \%$ & $0.0 \%$ & 1.00296 & \\
4 & $1.4 \%$ & $98.1 \%$ & $0.3 \%$ & $0.0 \%$ & $0.3 \%$ & $0.0 \%$ & 1.03949 & \\
5 & $1.4 \%$ & $98.1 \%$ & $0.1 \%$ & $0.3 \%$ & $0.1 \%$ & $0.0 \%$ & 1.03949 & \\
6 & $0.0 \%$ & $0.0 \%$ & $0.0 \%$ & $0.0 \%$ & $0.0 \%$ & $100.0 \%$ & 0.99991 & \\
7 & $0.0 \%$ & $0.0 \%$ & $0.0 \%$ & $0.0 \%$ & $0.0 \%$ & $100.0 \%$ & 1.00069 & \\
8 & $0.2 \%$ & $0.0 \%$ & $0.1 \%$ & $0.2 \%$ & $0.4 \%$ & $99.1 \%$ & 1.01753 & \\
9 & $0.2 \%$ & $0.0 \%$ & $0.3 \%$ & $0.2 \%$ & $0.1 \%$ & $99.1 \%$ & 1.01754 & \\
10 & $1.5 \%$ & $0.3 \%$ & $0.4 \%$ & $0.4 \%$ & $0.4 \%$ & $97.0 \%$ & 1.06260 & \\
11 & $18.8 \%$ & $80.5 \%$ & $0.2 \%$ & $0.2 \%$ & $0.2 \%$ & $0.1 \%$ & 1.46521 & \\
12 & $51.2 \%$ & $1.9 \%$ & $44.8 \%$ & $0.7 \%$ & $0.7 \%$ & $0.8 \%$ & 2.16129 & \\
13 & $51.2 \%$ & $1.9 \%$ & $0.7 \%$ & $44.8 \%$ & $0.7 \%$ & $0.8 \%$ & 2.16142 & \\
14 & $51.1 \%$ & $1.9 \%$ & $0.7 \%$ & $0.7 \%$ & $44.8 \%$ & $0.8 \%$ & 2.16208 & \\
\hline \hline
\end{tabular}

\section{S1.5.6 IRC Step 6}

\begin{tabular}{|c|c|c|}
\hline \multicolumn{3}{|c|}{1 1-center FNO analysis, $\varepsilon=0.85000$} \\
\hline $\mathrm{MO}$ & $\lambda$ & Atoms \\
\hline 1 & 1.00178 & $\mathrm{C}_{1}$ \\
\hline 2 & 1.00022 & $\mathrm{~F}_{2}$ \\
\hline 3 & 0.99852 & $\mathrm{~F}_{2}$ \\
\hline 4 & 0.98074 & $\mathrm{~F}_{2}$ \\
\hline 5 & 0.98073 & $\mathrm{~F}_{2}$ \\
\hline 6 & 1.00005 & $\mathrm{~F}_{6}$ \\
\hline 7 & 0.99962 & $\mathrm{~F}_{6}$ \\
\hline 8 & 0.99101 & $\mathrm{~F}_{6}$ \\
\hline 9 & 0.99099 & $\mathrm{~F}_{6}$ \\
\hline 10 & 0.96882 & $\mathrm{~F}_{6}$ \\
\hline \multicolumn{3}{|c|}{2 -center FNO analysis, $\varepsilon=0.85000$} \\
\hline MO & $\lambda$ & Atoms \\
\hline 11 & 0.99226 & $\mathrm{C}_{1}-\mathrm{F}_{2}$ \\
\hline 12 & 0.95884 & $\mathrm{C}_{1}-\mathrm{H}_{3}$ \\
\hline 13 & 0.95906 & $\mathrm{C}_{1}-\mathrm{H}_{4}$ \\
\hline 14 & 0.95858 & $\mathrm{C}_{1}-\mathrm{H}_{5}$ \\
\hline
\end{tabular}

\begin{tabular}{crrrrrrrr}
\hline \multicolumn{2}{c}{ Localization and atoms expanded by each FNO } \\
\hline \multicolumn{1}{r}{ FNO } & Loc $_{1}$ & $\mathrm{Loc}_{2}$ & $\mathrm{Loc}_{3}$ & $\mathrm{Loc}_{4}$ & $\mathrm{Loc}_{5}$ & $\mathrm{Loc}_{6}$ & $\mathrm{Loc}_{7}$ & $n_{\text {eff }}$ \\
\hline 1 & $100.2 \%$ & $0.0 \%$ & $0.0 \%$ & $0.0 \%$ & $0.0 \%$ & $0.0 \%$ & 0.99645 & \\
2 & $0.0 \%$ & $100.0 \%$ & $0.0 \%$ & $0.0 \%$ & $0.0 \%$ & $0.0 \%$ & 0.99957 & \\
3 & $0.1 \%$ & $99.9 \%$ & $0.0 \%$ & $0.0 \%$ & $0.0 \%$ & $0.0 \%$ & 1.00296 & \\
4 & $1.3 \%$ & $98.1 \%$ & $0.0 \%$ & $0.3 \%$ & $0.3 \%$ & $0.0 \%$ & 1.03945 & \\
5 & $1.4 \%$ & $98.1 \%$ & $0.3 \%$ & $0.1 \%$ & $0.1 \%$ & $0.0 \%$ & 1.03947 & \\
6 & $0.0 \%$ & $0.0 \%$ & $0.0 \%$ & $0.0 \%$ & $0.0 \%$ & $100.0 \%$ & 0.99990 & \\
7 & $0.0 \%$ & $0.0 \%$ & $0.0 \%$ & $0.0 \%$ & $0.0 \%$ & $100.0 \%$ & 1.00076 & \\
8 & $0.2 \%$ & $0.0 \%$ & $0.4 \%$ & $0.2 \%$ & $0.1 \%$ & $99.1 \%$ & 1.01821 & \\
9 & $0.2 \%$ & $0.0 \%$ & $0.1 \%$ & $0.3 \%$ & $0.3 \%$ & $99.1 \%$ & 1.01824 & \\
10 & $1.6 \%$ & $0.3 \%$ & $0.4 \%$ & $0.4 \%$ & $0.4 \%$ & $96.9 \%$ & 1.06507 & \\
11 & $18.7 \%$ & $80.5 \%$ & $0.2 \%$ & $0.2 \%$ & $0.2 \%$ & $0.1 \%$ & 1.46427 & \\
12 & $51.1 \%$ & $2.0 \%$ & $44.8 \%$ & $0.7 \%$ & $0.7 \%$ & $0.8 \%$ & 2.16348 & \\
13 & $51.1 \%$ & $2.0 \%$ & $0.7 \%$ & $44.8 \%$ & $0.7 \%$ & $0.8 \%$ & 2.16249 & \\
14 & $51.1 \%$ & $2.0 \%$ & $0.7 \%$ & $0.7 \%$ & $44.8 \%$ & $0.8 \%$ & 2.16461 & \\
\hline \hline
\end{tabular}


S1.5.7 IRC Step 7

\begin{tabular}{ccc}
\hline \hline \multicolumn{3}{c}{ 1-center FNO } \\
\hline MO & $\lambda$ & Atoms \\
\hline 1 & 1.00178 & $\mathrm{C}_{1}$ \\
2 & 1.00022 & $\mathrm{~F}_{2}$ \\
3 & 0.99852 & $\mathrm{~F}_{2}$ \\
4 & 0.98075 & $\mathrm{~F}_{2}$ \\
5 & 0.98074 & $\mathrm{~F}_{2}$ \\
6 & 1.00005 & $\mathrm{~F}_{6}$ \\
7 & 0.99958 & $\mathrm{~F}_{6}$ \\
8 & 0.99066 & $\mathrm{~F}_{6}$ \\
9 & 0.99065 & $\mathrm{~F}_{6}$ \\
10 & 0.96766 & $\mathrm{~F}_{6}$ \\
\hline 2 -center FNO analysis, $\varepsilon=0.85000$ \\
\hline MO & $\lambda$ & Atoms \\
\hline 11 & 0.99261 & $\mathrm{C}_{1}-\mathrm{F}_{2}$ \\
12 & 0.95861 & $\mathrm{C}_{1}-\mathrm{H}_{3}$ \\
13 & 0.95867 & $\mathrm{C}_{1}-\mathrm{H}_{4}$ \\
14 & 0.95861 & $\mathrm{C}_{1}-\mathrm{H}_{5}$ \\
\hline \hline
\end{tabular}

\begin{tabular}{crrrrrrrr}
\hline \hline \multicolumn{2}{l}{ Localization and atoms expanded by each FNO } \\
\hline \multicolumn{1}{r}{ FNO } & Loc $_{1}$ & $\mathrm{Loc}_{2}$ & $\mathrm{Loc}_{3}$ & $\mathrm{Loc}_{4}$ & $\mathrm{Loc}_{5}$ & $\mathrm{Loc}_{6}$ & $\mathrm{Loc}_{7}$ & $n_{\text {eff }}$ \\
\hline 1 & $100.2 \%$ & $0.0 \%$ & $0.0 \%$ & $0.0 \%$ & $0.0 \%$ & $0.0 \%$ & 0.99645 & \\
2 & $0.0 \%$ & $100.0 \%$ & $0.0 \%$ & $0.0 \%$ & $0.0 \%$ & $0.0 \%$ & 0.99957 & \\
3 & $0.1 \%$ & $99.9 \%$ & $0.0 \%$ & $0.0 \%$ & $0.0 \%$ & $0.0 \%$ & 1.00296 & \\
4 & $1.3 \%$ & $98.1 \%$ & $0.3 \%$ & $0.1 \%$ & $0.1 \%$ & $0.0 \%$ & 1.03942 & \\
5 & $1.4 \%$ & $98.1 \%$ & $0.0 \%$ & $0.3 \%$ & $0.2 \%$ & $0.0 \%$ & 1.03944 & \\
6 & $0.0 \%$ & $0.0 \%$ & $0.0 \%$ & $0.0 \%$ & $0.0 \%$ & $100.0 \%$ & 0.99990 & \\
7 & $0.0 \%$ & $0.0 \%$ & $0.0 \%$ & $0.0 \%$ & $0.0 \%$ & $100.0 \%$ & 1.00083 & \\
8 & $0.2 \%$ & $0.0 \%$ & $0.4 \%$ & $0.2 \%$ & $0.1 \%$ & $99.1 \%$ & 1.01892 & \\
9 & $0.2 \%$ & $0.0 \%$ & $0.1 \%$ & $0.3 \%$ & $0.4 \%$ & $99.1 \%$ & 1.01894 & \\
10 & $1.6 \%$ & $0.3 \%$ & $0.4 \%$ & $0.4 \%$ & $0.4 \%$ & $96.8 \%$ & 1.06759 & \\
11 & $18.7 \%$ & $80.5 \%$ & $0.2 \%$ & $0.2 \%$ & $0.2 \%$ & $0.1 \%$ & 1.46365 & \\
12 & $51.1 \%$ & $2.0 \%$ & $44.8 \%$ & $0.7 \%$ & $0.7 \%$ & $0.8 \%$ & 2.16467 & \\
13 & $51.1 \%$ & $2.0 \%$ & $0.7 \%$ & $44.8 \%$ & $0.7 \%$ & $0.8 \%$ & 2.16441 & \\
14 & $51.1 \%$ & $2.0 \%$ & $0.7 \%$ & $0.7 \%$ & $44.8 \%$ & $0.8 \%$ & 2.16460 & \\
\hline \hline
\end{tabular}

S1.5.8 IRC Step 8

\begin{tabular}{|c|c|c|}
\hline \multicolumn{3}{|c|}{ 1-center FNO analysis, $\varepsilon=0.85000$} \\
\hline $\mathrm{MO}$ & $\lambda$ & Atoms \\
\hline 1 & 1.00178 & $\mathrm{C}_{1}$ \\
\hline 2 & 1.00022 & $\mathrm{~F}_{2}$ \\
\hline 3 & 0.99852 & $\mathrm{~F}_{2}$ \\
\hline 4 & 0.98074 & $\mathrm{~F}_{2}$ \\
\hline 5 & 0.98074 & $\mathrm{~F}_{2}$ \\
\hline 6 & 1.00005 & $\mathrm{~F}_{6}$ \\
\hline 7 & 0.99954 & $\mathrm{~F}_{6}$ \\
\hline 8 & 0.99031 & $\mathrm{~F}_{6}$ \\
\hline 9 & 0.99030 & $\mathrm{~F}_{6}$ \\
\hline 10 & 0.96634 & $\mathrm{~F}_{6}$ \\
\hline \multicolumn{3}{|c|}{ 2-center FNO analysis, $\varepsilon=0.85000$} \\
\hline $\mathrm{MO}$ & $\lambda$ & Atoms \\
\hline 11 & 0.99206 & $\mathrm{C}_{1}-\mathrm{F}_{2}$ \\
\hline 12 & 0.95838 & $\mathrm{C}_{1}-\mathrm{H}_{3}$ \\
\hline 13 & 0.95850 & $\mathrm{C}_{1}-\mathrm{H}_{4}$ \\
\hline 14 & 0.95846 & $\mathrm{C}_{1}-\mathrm{H}_{5}$ \\
\hline
\end{tabular}




\begin{tabular}{crrrrrrrr}
\hline \hline \multicolumn{2}{l}{ Localization and atoms expanded by each FNO } \\
\hline FNO & Loc $_{1}$ & \multicolumn{1}{l}{ Loc $_{2}$} & Loc $_{3}$ & Loc $_{4}$ & Loc $_{5}$ & Loc $_{6}$ & Loc $_{7}$ & $n_{\text {eff }}$ \\
\hline 1 & $100.2 \%$ & $0.0 \%$ & $0.0 \%$ & $0.0 \%$ & $0.0 \%$ & $0.0 \%$ & 0.99644 & \\
2 & $0.0 \%$ & $100.0 \%$ & $0.0 \%$ & $0.0 \%$ & $0.0 \%$ & $0.0 \%$ & 0.99957 & \\
3 & $0.1 \%$ & $99.9 \%$ & $0.0 \%$ & $0.0 \%$ & $0.0 \%$ & $0.0 \%$ & 1.00296 & \\
4 & $1.3 \%$ & $98.1 \%$ & $0.1 \%$ & $0.3 \%$ & $0.2 \%$ & $0.0 \%$ & 1.03945 & \\
5 & $1.3 \%$ & $98.1 \%$ & $0.3 \%$ & $0.0 \%$ & $0.2 \%$ & $0.0 \%$ & 1.03946 & \\
6 & $0.0 \%$ & $0.0 \%$ & $0.0 \%$ & $0.0 \%$ & $0.0 \%$ & $100.0 \%$ & 0.99989 & \\
7 & $0.0 \%$ & $0.0 \%$ & $0.0 \%$ & $0.0 \%$ & $0.0 \%$ & $100.0 \%$ & 1.00091 & \\
8 & $0.2 \%$ & $0.0 \%$ & $0.4 \%$ & $0.2 \%$ & $0.2 \%$ & $99.0 \%$ & 1.01964 & \\
9 & $0.2 \%$ & $0.0 \%$ & $0.1 \%$ & $0.3 \%$ & $0.3 \%$ & $99.0 \%$ & 1.01967 & \\
10 & $1.7 \%$ & $0.3 \%$ & $0.4 \%$ & $0.4 \%$ & $0.4 \%$ & $96.6 \%$ & 1.07046 & \\
11 & $18.7 \%$ & $80.5 \%$ & $0.2 \%$ & $0.2 \%$ & $0.2 \%$ & $0.1 \%$ & 1.46277 & \\
12 & $51.0 \%$ & $2.0 \%$ & $44.8 \%$ & $0.7 \%$ & $0.7 \%$ & $0.9 \%$ & 2.16587 & \\
13 & $51.0 \%$ & $2.0 \%$ & $0.7 \%$ & $44.8 \%$ & $0.7 \%$ & $0.9 \%$ & 2.16536 & \\
14 & $51.0 \%$ & $2.0 \%$ & $0.7 \%$ & $0.7 \%$ & $44.8 \%$ & $0.8 \%$ & 2.16539 & \\
\hline \hline
\end{tabular}

\section{S1.5.9 IRC Step 9}

\begin{tabular}{|c|c|c|}
\hline \multicolumn{3}{|c|}{1 1-center FNO analysis, $\varepsilon=0.85000$} \\
\hline $\mathrm{MO}$ & $\lambda$ & Atoms \\
\hline 1 & 1.00179 & $\mathrm{C}_{1}$ \\
\hline 2 & 1.00022 & $\mathrm{~F}_{2}$ \\
\hline 3 & 0.99852 & $\mathrm{~F}_{2}$ \\
\hline 4 & 0.98076 & $\mathrm{~F}_{2}$ \\
\hline 5 & 0.98075 & $\mathrm{~F}_{2}$ \\
\hline 6 & 1.00006 & $\mathrm{~F}_{6}$ \\
\hline 7 & 0.99950 & $\mathrm{~F}_{6}$ \\
\hline 8 & 0.98993 & $\mathrm{~F}_{6}$ \\
\hline 9 & 0.98993 & $\mathrm{~F}_{6}$ \\
\hline 10 & 0.96499 & $\mathrm{~F}_{6}$ \\
\hline \multicolumn{3}{|c|}{2 -center FNO analysis, $\varepsilon=0.85000$} \\
\hline MO & $\lambda$ & Atoms \\
\hline 11 & 0.99205 & $\mathrm{C}_{1}-\mathrm{F}_{2}$ \\
\hline 12 & 0.95834 & $\mathrm{C}_{1}-\mathrm{H}_{3}$ \\
\hline 13 & 0.95828 & $\mathrm{C}_{1}-\mathrm{H}_{4}$ \\
\hline 14 & 0.95804 & $\mathrm{C}_{1}-\mathrm{H}_{5}$ \\
\hline
\end{tabular}

\begin{tabular}{crrrrrrrr}
\hline \multicolumn{2}{c}{ Localization and atoms expanded by each FNO } \\
\hline \multicolumn{1}{r}{ FNO } & Loc $_{1}$ & $\mathrm{Loc}_{2}$ & $\mathrm{Loc}_{3}$ & $\mathrm{Loc}_{4}$ & $\mathrm{Loc}_{5}$ & $\mathrm{Loc}_{6}$ & $\mathrm{Loc}_{7}$ & $n_{\text {eff }}$ \\
\hline 1 & $100.2 \%$ & $0.0 \%$ & $0.0 \%$ & $0.0 \%$ & $0.0 \%$ & $0.0 \%$ & 0.99644 & \\
2 & $0.0 \%$ & $100.0 \%$ & $0.0 \%$ & $0.0 \%$ & $0.0 \%$ & $0.0 \%$ & 0.99957 & \\
3 & $0.1 \%$ & $99.9 \%$ & $0.0 \%$ & $0.0 \%$ & $0.0 \%$ & $0.0 \%$ & 1.00297 & \\
4 & $1.3 \%$ & $98.1 \%$ & $0.3 \%$ & $0.0 \%$ & $0.2 \%$ & $0.0 \%$ & 1.03941 & \\
5 & $1.3 \%$ & $98.1 \%$ & $0.0 \%$ & $0.3 \%$ & $0.2 \%$ & $0.0 \%$ & 1.03943 & \\
6 & $0.0 \%$ & $0.0 \%$ & $0.0 \%$ & $0.0 \%$ & $0.0 \%$ & $100.0 \%$ & 0.99989 & \\
7 & $0.0 \%$ & $0.0 \%$ & $0.0 \%$ & $0.0 \%$ & $0.0 \%$ & $100.0 \%$ & 1.00100 & \\
8 & $0.2 \%$ & $0.0 \%$ & $0.1 \%$ & $0.3 \%$ & $0.4 \%$ & $99.0 \%$ & 1.02041 & \\
9 & $0.2 \%$ & $0.0 \%$ & $0.4 \%$ & $0.2 \%$ & $0.1 \%$ & $99.0 \%$ & 1.02042 & \\
10 & $1.8 \%$ & $0.4 \%$ & $0.4 \%$ & $0.4 \%$ & $0.4 \%$ & $96.5 \%$ & 1.07341 & \\
11 & $18.6 \%$ & $80.6 \%$ & $0.2 \%$ & $0.2 \%$ & $0.2 \%$ & $0.1 \%$ & 1.46193 & \\
12 & $51.0 \%$ & $2.0 \%$ & $44.8 \%$ & $0.7 \%$ & $0.7 \%$ & $0.9 \%$ & 2.16616 & \\
13 & $51.0 \%$ & $2.0 \%$ & $0.7 \%$ & $44.8 \%$ & $0.7 \%$ & $0.9 \%$ & 2.16648 & \\
14 & $51.0 \%$ & $2.0 \%$ & $0.7 \%$ & $0.7 \%$ & $44.8 \%$ & $0.9 \%$ & 2.16759 & \\
\hline \hline
\end{tabular}


S1.5.10 IRC Step 10

\begin{tabular}{ccc}
\hline \hline \multicolumn{3}{c}{ 1-center FNO analysis, $\varepsilon=0.85000$} \\
\hline MO & $\lambda$ & Atoms \\
\hline 1 & 1.00179 & $\mathrm{C}_{1}$ \\
2 & 1.00022 & $\mathrm{~F}_{2}$ \\
3 & 0.99852 & $\mathrm{~F}_{2}$ \\
4 & 0.98080 & $\mathrm{~F}_{2}$ \\
5 & 0.98077 & $\mathrm{~F}_{2}$ \\
6 & 1.00006 & $\mathrm{~F}_{6}$ \\
7 & 0.99946 & $\mathrm{~F}_{6}$ \\
8 & 0.98956 & $\mathrm{~F}_{6}$ \\
9 & 0.98956 & $\mathrm{~F}_{6}$ \\
10 & 0.96340 & $\mathrm{~F}_{6}$ \\
\hline 2 -center FNO & analysis, $\varepsilon=0.85000$ \\
\hline MO & $\lambda$ & Atoms \\
\hline 11 & 0.99196 & $\mathrm{C}_{1}-\mathrm{F}_{2}$ \\
12 & 0.95769 & $\mathrm{C}_{1}-\mathrm{H}_{3}$ \\
13 & 0.95791 & $\mathrm{C}_{1}-\mathrm{H}_{4}$ \\
14 & 0.95787 & $\mathrm{C}_{1}-\mathrm{H}_{5}$ \\
\hline \hline
\end{tabular}

\begin{tabular}{crrrrrrrr}
\hline \hline \multicolumn{7}{l}{ Localization and atoms expanded by each FNO } & & \\
\hline \hline FNO & Loc $_{1}$ & Loc $_{2}$ & Loc $_{3}$ & Loc $_{4}$ & Loc $_{5}$ & Loc $_{6}$ & Loc $_{7}$ & $n_{\text {eff }}$ \\
\hline 1 & $100.2 \%$ & $0.0 \%$ & $0.0 \%$ & $0.0 \%$ & $0.0 \%$ & $0.0 \%$ & 0.99643 & \\
2 & $0.0 \%$ & $100.0 \%$ & $0.0 \%$ & $0.0 \%$ & $0.0 \%$ & $0.0 \%$ & 0.99957 & \\
3 & $0.1 \%$ & $99.9 \%$ & $0.0 \%$ & $0.0 \%$ & $0.0 \%$ & $0.0 \%$ & 1.00298 & \\
4 & $1.3 \%$ & $98.1 \%$ & $0.0 \%$ & $0.2 \%$ & $0.3 \%$ & $0.0 \%$ & 1.03934 & \\
5 & $1.3 \%$ & $98.1 \%$ & $0.3 \%$ & $0.1 \%$ & $0.1 \%$ & $0.0 \%$ & 1.03940 & \\
6 & $0.0 \%$ & $0.0 \%$ & $0.0 \%$ & $0.0 \%$ & $0.0 \%$ & $100.0 \%$ & 0.99988 & \\
7 & $0.0 \%$ & $0.0 \%$ & $0.0 \%$ & $0.0 \%$ & $0.0 \%$ & $99.9 \%$ & 1.00109 & \\
8 & $0.2 \%$ & $0.0 \%$ & $0.3 \%$ & $0.1 \%$ & $0.4 \%$ & $99.0 \%$ & 1.02117 & \\
9 & $0.2 \%$ & $0.0 \%$ & $0.2 \%$ & $0.4 \%$ & $0.2 \%$ & $99.0 \%$ & 1.02118 & \\
10 & $1.9 \%$ & $0.4 \%$ & $0.4 \%$ & $0.4 \%$ & $0.4 \%$ & $96.3 \%$ & 1.07690 & \\
11 & $18.6 \%$ & $80.6 \%$ & $0.2 \%$ & $0.2 \%$ & $0.2 \%$ & $0.1 \%$ & 1.46125 & \\
12 & $50.9 \%$ & $2.0 \%$ & $44.9 \%$ & $0.6 \%$ & $0.6 \%$ & $0.9 \%$ & 2.16936 & \\
13 & $50.9 \%$ & $2.0 \%$ & $0.6 \%$ & $44.9 \%$ & $0.7 \%$ & $0.9 \%$ & 2.16832 & \\
14 & $50.9 \%$ & $2.0 \%$ & $0.6 \%$ & $0.6 \%$ & $44.9 \%$ & $0.9 \%$ & 2.16847 & \\
\hline \hline
\end{tabular}

\section{S1.5.11 IRC Step 11}

\begin{tabular}{ccc}
\hline \multicolumn{3}{c}{ 1-center FNO analysis, $\varepsilon=0.85000$} \\
\hline MO & $\lambda$ & Atoms \\
\hline 1 & 1.00180 & $\mathrm{C}_{1}$ \\
2 & 1.00022 & $\mathrm{~F}_{2}$ \\
3 & 0.99851 & $\mathrm{~F}_{2}$ \\
4 & 0.98089 & $\mathrm{~F}_{2}$ \\
5 & 0.98088 & $\mathrm{~F}_{2}$ \\
6 & 1.00006 & $\mathrm{~F}_{6}$ \\
7 & 0.99940 & $\mathrm{~F}_{6}$ \\
8 & 0.98918 & $\mathrm{~F}_{6}$ \\
9 & 0.98917 & $\mathrm{~F}_{6}$ \\
10 & 0.96156 & $\mathrm{~F}_{6}$ \\
\hline 2 -center $\mathrm{FNO}$ analysis, $\varepsilon=0.85000$ \\
\hline MO & $\lambda$ & Atoms \\
\hline 11 & 0.99183 & $\mathrm{C}_{1}-\mathrm{F}_{2}$ \\
12 & 0.95800 & $\mathrm{C}_{1}-\mathrm{H}_{3}$ \\
13 & 0.95792 & $\mathrm{C}_{1}-\mathrm{H}_{4}$ \\
14 & 0.95759 & $\mathrm{C}_{1}-\mathrm{H}_{5}$ \\
\hline \hline
\end{tabular}




\begin{tabular}{crrrrrrrr}
\hline \hline \multicolumn{2}{l}{ Localization and atoms expanded by each FNO } \\
\hline FNO & Loc $_{1}$ & \multicolumn{1}{l}{ Loc $_{2}$} & Loc $_{3}$ & Loc $_{4}$ & Loc $_{5}$ & Loc $_{6}$ & Loc $_{7}$ & $n_{\text {eff }}$ \\
\hline 1 & $100.2 \%$ & $0.0 \%$ & $0.0 \%$ & $0.0 \%$ & $0.0 \%$ & $0.0 \%$ & 0.99640 & \\
2 & $0.0 \%$ & $100.0 \%$ & $0.0 \%$ & $0.0 \%$ & $0.0 \%$ & $0.0 \%$ & 0.99957 & \\
3 & $0.1 \%$ & $99.9 \%$ & $0.0 \%$ & $0.0 \%$ & $0.0 \%$ & $0.0 \%$ & 1.00299 & \\
4 & $1.3 \%$ & $98.1 \%$ & $0.1 \%$ & $0.1 \%$ & $0.3 \%$ & $0.0 \%$ & 1.03914 & \\
5 & $1.3 \%$ & $98.1 \%$ & $0.3 \%$ & $0.2 \%$ & $0.0 \%$ & $0.0 \%$ & 1.03917 & \\
6 & $0.0 \%$ & $0.0 \%$ & $0.0 \%$ & $0.0 \%$ & $0.0 \%$ & $100.0 \%$ & 0.99988 & \\
7 & $0.0 \%$ & $0.0 \%$ & $0.0 \%$ & $0.0 \%$ & $0.0 \%$ & $99.9 \%$ & 1.00119 & \\
8 & $0.2 \%$ & $0.0 \%$ & $0.1 \%$ & $0.4 \%$ & $0.2 \%$ & $98.9 \%$ & 1.02196 & \\
9 & $0.2 \%$ & $0.0 \%$ & $0.4 \%$ & $0.1 \%$ & $0.3 \%$ & $98.9 \%$ & 1.02198 & \\
10 & $2.1 \%$ & $0.4 \%$ & $0.5 \%$ & $0.5 \%$ & $0.5 \%$ & $96.2 \%$ & 1.08097 & \\
11 & $18.6 \%$ & $80.6 \%$ & $0.2 \%$ & $0.2 \%$ & $0.2 \%$ & $0.1 \%$ & 1.46167 & \\
12 & $50.9 \%$ & $2.0 \%$ & $44.9 \%$ & $0.6 \%$ & $0.6 \%$ & $0.9 \%$ & 2.16792 & \\
13 & $50.9 \%$ & $2.0 \%$ & $0.6 \%$ & $44.9 \%$ & $0.6 \%$ & $0.9 \%$ & 2.16838 & \\
14 & $50.9 \%$ & $2.0 \%$ & $0.6 \%$ & $0.6 \%$ & $44.9 \%$ & $0.9 \%$ & 2.16996 & \\
\hline \hline
\end{tabular}

\section{S1.5.12 IRC Step 12}

\begin{tabular}{ccc}
\hline \hline \multicolumn{3}{c}{ 1-center FNO analysis, $\varepsilon=0.85000$} \\
\hline MO & $\lambda$ & Atoms \\
\hline 1 & 1.00183 & $\mathrm{C}_{1}$ \\
2 & 1.00021 & $\mathrm{~F}_{2}$ \\
3 & 0.99850 & $\mathrm{~F}_{2}$ \\
4 & 0.98112 & $\mathrm{~F}_{2}$ \\
5 & 0.98111 & $\mathrm{~F}_{2}$ \\
6 & 1.00006 & $\mathrm{~F}_{6}$ \\
7 & 0.99934 & $\mathrm{~F}_{6}$ \\
8 & 0.98882 & $\mathrm{~F}_{6}$ \\
9 & 0.98881 & $\mathrm{~F}_{6}$ \\
10 & 0.95932 & $\mathrm{~F}_{6}$ \\
\hline 2 -center FNO analysis, $\varepsilon=0.85000$ \\
\hline MO & $\lambda$ & Atoms $_{1}$ \\
\hline 11 & 0.99171 & $\mathrm{C}_{1}-\mathrm{F}_{2}$ \\
12 & 0.95775 & $\mathrm{C}_{1}-\mathrm{H}_{3}$ \\
13 & 0.95785 & $\mathrm{C}_{1}-\mathrm{H}_{4}$ \\
14 & 0.95755 & $\mathrm{C}_{1}-\mathrm{H}_{5}$ \\
\hline \hline
\end{tabular}

\begin{tabular}{crrrrrrrr}
\hline \hline \multicolumn{2}{l}{ Localization and atoms expanded by each FNO } & & & \\
\hline FNO & Loc $_{1}$ & $\mathrm{Loc}_{2}$ & $\mathrm{Loc}_{3}$ & Loc $_{4}$ & $\mathrm{Loc}_{5}$ & $\mathrm{Loc}_{6}$ & $\mathrm{Loc}_{7}$ & $n_{\text {eff }}$ \\
\hline 1 & $100.2 \%$ & $0.0 \%$ & $0.0 \%$ & $0.0 \%$ & $0.0 \%$ & $0.0 \%$ & 0.99636 & \\
2 & $0.0 \%$ & $100.0 \%$ & $0.0 \%$ & $0.0 \%$ & $0.0 \%$ & $0.0 \%$ & 0.99957 & \\
3 & $0.1 \%$ & $99.9 \%$ & $0.0 \%$ & $0.0 \%$ & $0.0 \%$ & $0.0 \%$ & 1.00300 & \\
4 & $1.3 \%$ & $98.1 \%$ & $0.3 \%$ & $0.0 \%$ & $0.2 \%$ & $0.0 \%$ & 1.03865 & \\
5 & $1.3 \%$ & $98.1 \%$ & $0.0 \%$ & $0.3 \%$ & $0.2 \%$ & $0.0 \%$ & 1.03868 & \\
6 & $0.0 \%$ & $0.0 \%$ & $0.0 \%$ & $0.0 \%$ & $0.0 \%$ & $100.0 \%$ & 0.99987 & \\
7 & $0.0 \%$ & $0.0 \%$ & $0.0 \%$ & $0.0 \%$ & $0.0 \%$ & $99.9 \%$ & 1.00131 & \\
8 & $0.3 \%$ & $0.0 \%$ & $0.1 \%$ & $0.3 \%$ & $0.4 \%$ & $98.9 \%$ & 1.02271 & \\
9 & $0.3 \%$ & $0.0 \%$ & $0.4 \%$ & $0.2 \%$ & $0.1 \%$ & $98.9 \%$ & 1.02273 & \\
10 & $2.2 \%$ & $0.5 \%$ & $0.5 \%$ & $0.5 \%$ & $0.5 \%$ & $95.9 \%$ & 1.08592 & \\
11 & $18.6 \%$ & $80.6 \%$ & $0.2 \%$ & $0.2 \%$ & $0.2 \%$ & $0.1 \%$ & 1.46235 & \\
12 & $50.9 \%$ & $1.9 \%$ & $44.9 \%$ & $0.6 \%$ & $0.6 \%$ & $1.0 \%$ & 2.16909 & \\
13 & $50.9 \%$ & $1.9 \%$ & $0.6 \%$ & $44.9 \%$ & $0.6 \%$ & $1.0 \%$ & 2.16863 & \\
14 & $50.9 \%$ & $1.9 \%$ & $0.6 \%$ & $0.6 \%$ & $44.9 \%$ & $1.0 \%$ & 2.17005 & \\
\hline \hline
\end{tabular}


S1.5.13 IRC Step 13

\begin{tabular}{ccc}
\hline \hline \multicolumn{3}{c}{ 1-center FNO analysis, $\varepsilon=0.85000$} \\
\hline MO & $\lambda$ & Atoms \\
\hline 1 & 1.00185 & $\mathrm{C}_{1}$ \\
2 & 1.00021 & $\mathrm{~F}_{2}$ \\
3 & 0.99849 & $\mathrm{~F}_{2}$ \\
4 & 0.98147 & $\mathrm{~F}_{2}$ \\
5 & 0.98145 & $\mathrm{~F}_{2}$ \\
6 & 1.00007 & $\mathrm{~F}_{6}$ \\
7 & 0.99927 & $\mathrm{~F}_{6}$ \\
8 & 0.98847 & $\mathrm{~F}_{6}$ \\
9 & 0.98847 & $\mathrm{~F}_{6}$ \\
10 & 0.95631 & $\mathrm{~F}_{6}$ \\
\hline 2 -center FNO analysis, $=0.85000$ \\
\hline MO & \multicolumn{3}{c}{$\lambda$} & Atoms \\
\hline 11 & 0.99105 & $\mathrm{C}_{1}-\mathrm{F}_{2}$ \\
12 & 0.95830 & $\mathrm{C}_{1}-\mathrm{H}_{3}$ \\
13 & 0.95805 & $\mathrm{C}_{1}-\mathrm{H}_{4}$ \\
14 & 0.95800 & $\mathrm{C}_{1}-\mathrm{H}_{5}$ \\
\hline \hline
\end{tabular}

\begin{tabular}{crrrrrrrr}
\hline \multicolumn{2}{l}{ Localization and atoms expanded by each FNO } & & & \\
\hline FNO & Loc $_{1}$ & Loc $_{2}$ & Loc $_{3}$ & Loc $_{4}$ & Loc $_{5}$ & Loc $_{6}$ & Loc $_{7}$ & $n_{\text {eff }}$ \\
\hline 1 & $100.2 \%$ & $0.0 \%$ & $0.0 \%$ & $0.0 \%$ & $0.0 \%$ & $0.0 \%$ & 0.99631 & \\
2 & $0.0 \%$ & $100.0 \%$ & $0.0 \%$ & $0.0 \%$ & $0.0 \%$ & $0.0 \%$ & 0.99958 & \\
3 & $0.1 \%$ & $99.8 \%$ & $0.0 \%$ & $0.0 \%$ & $0.0 \%$ & $0.0 \%$ & 1.00303 & \\
4 & $1.3 \%$ & $98.1 \%$ & $0.2 \%$ & $0.0 \%$ & $0.3 \%$ & $0.0 \%$ & 1.03792 & \\
5 & $1.3 \%$ & $98.1 \%$ & $0.1 \%$ & $0.3 \%$ & $0.1 \%$ & $0.0 \%$ & 1.03797 & \\
6 & $0.0 \%$ & $0.0 \%$ & $0.0 \%$ & $0.0 \%$ & $0.0 \%$ & $100.0 \%$ & 0.99986 & \\
7 & $0.0 \%$ & $0.0 \%$ & $0.0 \%$ & $0.0 \%$ & $0.0 \%$ & $99.9 \%$ & 1.00145 & \\
8 & $0.3 \%$ & $0.0 \%$ & $0.4 \%$ & $0.1 \%$ & $0.4 \%$ & $98.8 \%$ & 1.02342 & \\
9 & $0.3 \%$ & $0.0 \%$ & $0.2 \%$ & $0.5 \%$ & $0.2 \%$ & $98.8 \%$ & 1.02342 & \\
10 & $2.5 \%$ & $0.5 \%$ & $0.5 \%$ & $0.5 \%$ & $0.5 \%$ & $95.6 \%$ & 1.09264 & \\
11 & $18.6 \%$ & $80.5 \%$ & $0.2 \%$ & $0.2 \%$ & $0.2 \%$ & $0.1 \%$ & 1.46491 & \\
12 & $51.0 \%$ & $1.9 \%$ & $44.8 \%$ & $0.6 \%$ & $0.6 \%$ & $1.0 \%$ & 2.16626 & \\
13 & $51.0 \%$ & $1.9 \%$ & $0.6 \%$ & $44.8 \%$ & $0.6 \%$ & $1.0 \%$ & 2.16750 & \\
14 & $51.0 \%$ & $1.9 \%$ & $0.6 \%$ & $0.6 \%$ & $44.8 \%$ & $1.0 \%$ & 2.16772 & \\
\hline \hline
\end{tabular}

\section{S1.5.14 IRC Step 14}

\begin{tabular}{|c|c|c|}
\hline \multicolumn{3}{|c|}{ 1-center FNO analysis, $\varepsilon=0.85000$} \\
\hline $\mathrm{MO}$ & $\lambda$ & Atoms \\
\hline 1 & 1.00187 & $\mathrm{C}_{1}$ \\
\hline 2 & 1.00021 & $\mathrm{~F}_{2}$ \\
\hline 3 & 0.99847 & $\mathrm{~F}_{2}$ \\
\hline 4 & 0.98195 & $\mathrm{~F}_{2}$ \\
\hline 5 & 0.98193 & $\mathrm{~F}_{2}$ \\
\hline 6 & 1.00007 & $\mathrm{~F}_{6}$ \\
\hline 7 & 0.99920 & $\mathrm{~F}_{6}$ \\
\hline 8 & 0.98818 & $\mathrm{~F}_{6}$ \\
\hline 9 & 0.98817 & $\mathrm{~F}_{6}$ \\
\hline 10 & 0.95266 & $\mathrm{~F}_{6}$ \\
\hline \multicolumn{3}{|c|}{ 2-center FNO analysis, $\varepsilon=0.85000$} \\
\hline $\mathrm{MO}$ & $\lambda$ & Atoms \\
\hline 11 & 0.99055 & $\mathrm{C}_{1}-\mathrm{F}_{2}$ \\
\hline 12 & 0.95849 & $\mathrm{C}_{1}-\mathrm{H}_{3}$ \\
\hline 13 & 0.95901 & $\mathrm{C}_{1}-\mathrm{H}_{4}$ \\
\hline 14 & 0.95877 & $\mathrm{C}_{1}-\mathrm{H}_{5}$ \\
\hline
\end{tabular}




\begin{tabular}{crrrrrrrr}
\hline \hline \multicolumn{2}{l}{ Localization and atoms expanded by each FNO } \\
\hline FNO & Loc $_{1}$ & \multicolumn{1}{l}{ Loc $_{2}$} & Loc $_{3}$ & Loc $_{4}$ & Loc $_{5}$ & Loc $_{6}$ & Loc $_{7}$ & $n_{\text {eff }}$ \\
\hline 1 & $100.2 \%$ & $0.0 \%$ & $0.0 \%$ & $0.0 \%$ & $0.0 \%$ & $0.0 \%$ & 0.99627 & \\
2 & $0.0 \%$ & $100.0 \%$ & $0.0 \%$ & $0.0 \%$ & $0.0 \%$ & $0.0 \%$ & 0.99959 & \\
3 & $0.1 \%$ & $99.8 \%$ & $0.0 \%$ & $0.0 \%$ & $0.0 \%$ & $0.0 \%$ & 1.00307 & \\
4 & $1.2 \%$ & $98.2 \%$ & $0.0 \%$ & $0.2 \%$ & $0.3 \%$ & $0.0 \%$ & 1.03692 & \\
5 & $1.2 \%$ & $98.2 \%$ & $0.3 \%$ & $0.1 \%$ & $0.1 \%$ & $0.0 \%$ & 1.03697 & \\
6 & $0.0 \%$ & $0.0 \%$ & $0.0 \%$ & $0.0 \%$ & $0.0 \%$ & $100.0 \%$ & 0.99986 & \\
7 & $0.0 \%$ & $0.0 \%$ & $0.0 \%$ & $0.0 \%$ & $0.0 \%$ & $99.9 \%$ & 1.00161 & \\
8 & $0.3 \%$ & $0.0 \%$ & $0.2 \%$ & $0.2 \%$ & $0.5 \%$ & $98.8 \%$ & 1.02402 & \\
9 & $0.3 \%$ & $0.0 \%$ & $0.4 \%$ & $0.3 \%$ & $0.1 \%$ & $98.8 \%$ & 1.02405 & \\
10 & $2.8 \%$ & $0.6 \%$ & $0.5 \%$ & $0.5 \%$ & $0.5 \%$ & $95.3 \%$ & 1.10083 & \\
11 & $18.6 \%$ & $80.4 \%$ & $0.3 \%$ & $0.3 \%$ & $0.3 \%$ & $0.1 \%$ & 1.46736 & \\
12 & $51.1 \%$ & $1.8 \%$ & $44.8 \%$ & $0.6 \%$ & $0.6 \%$ & $1.1 \%$ & 2.16509 & \\
13 & $51.1 \%$ & $1.9 \%$ & $0.6 \%$ & $44.8 \%$ & $0.6 \%$ & $1.1 \%$ & 2.16266 & \\
14 & $51.1 \%$ & $1.9 \%$ & $0.6 \%$ & $0.6 \%$ & $44.8 \%$ & $1.1 \%$ & 2.16377 & \\
\hline \hline
\end{tabular}

S1.5.15 IRC Step 15

\begin{tabular}{|c|c|c|}
\hline \multicolumn{3}{|c|}{1 1-center FNO analysis, $\varepsilon=0.85000$} \\
\hline $\mathrm{MO}$ & $\lambda$ & Atoms \\
\hline 1 & 1.00187 & $\mathrm{C}_{1}$ \\
\hline 2 & 1.00020 & $\mathrm{~F}_{2}$ \\
\hline 3 & 0.99845 & $\mathrm{~F}_{2}$ \\
\hline 4 & 0.98251 & $\mathrm{~F}_{2}$ \\
\hline 5 & 0.98249 & $\mathrm{~F}_{2}$ \\
\hline 6 & 1.00008 & $\mathrm{~F}_{6}$ \\
\hline 7 & 0.99911 & $\mathrm{~F}_{6}$ \\
\hline 8 & 0.98795 & $\mathrm{~F}_{6}$ \\
\hline 9 & 0.98794 & $\mathrm{~F}_{6}$ \\
\hline 10 & 0.94813 & $\mathrm{~F}_{6}$ \\
\hline \multicolumn{3}{|c|}{2 -center FNO analysis, $\varepsilon=0.85000$} \\
\hline MO & $\lambda$ & Atoms \\
\hline 11 & 0.99021 & $\mathrm{C}_{1}-\mathrm{F}_{2}$ \\
\hline 12 & 0.95922 & $\mathrm{C}_{1}-\mathrm{H}_{3}$ \\
\hline 13 & 0.95902 & $\mathrm{C}_{1}-\mathrm{H}_{4}$ \\
\hline 14 & 0.95936 & $\mathrm{C}_{1}-\mathrm{H}_{5}$ \\
\hline
\end{tabular}

\begin{tabular}{crrrrrrrr}
\hline \multicolumn{2}{c}{ Localization and atoms expanded by each FNO } \\
\hline \multicolumn{1}{r}{ FNO } & Loc $_{1}$ & $\mathrm{Loc}_{2}$ & $\mathrm{Loc}_{3}$ & $\mathrm{Loc}_{4}$ & $\mathrm{Loc}_{5}$ & $\mathrm{Loc}_{6}$ & $\mathrm{Loc}_{7}$ & $n_{\text {eff }}$ \\
\hline 1 & $100.2 \%$ & $0.0 \%$ & $0.0 \%$ & $0.0 \%$ & $0.0 \%$ & $0.0 \%$ & 0.99628 & \\
2 & $0.0 \%$ & $100.0 \%$ & $0.0 \%$ & $0.0 \%$ & $0.0 \%$ & $0.0 \%$ & 0.99959 & \\
3 & $0.1 \%$ & $99.8 \%$ & $0.0 \%$ & $0.0 \%$ & $0.0 \%$ & $0.0 \%$ & 1.00311 & \\
4 & $1.2 \%$ & $98.3 \%$ & $0.2 \%$ & $0.3 \%$ & $0.1 \%$ & $0.0 \%$ & 1.03575 & \\
5 & $1.2 \%$ & $98.2 \%$ & $0.2 \%$ & $0.0 \%$ & $0.3 \%$ & $0.0 \%$ & 1.03579 & \\
6 & $0.0 \%$ & $0.0 \%$ & $0.0 \%$ & $0.0 \%$ & $0.0 \%$ & $100.0 \%$ & 0.99985 & \\
7 & $0.0 \%$ & $0.0 \%$ & $0.0 \%$ & $0.0 \%$ & $0.0 \%$ & $99.9 \%$ & 1.00177 & \\
8 & $0.3 \%$ & $0.0 \%$ & $0.2 \%$ & $0.2 \%$ & $0.5 \%$ & $98.8 \%$ & 1.02449 & \\
9 & $0.3 \%$ & $0.0 \%$ & $0.4 \%$ & $0.4 \%$ & $0.1 \%$ & $98.8 \%$ & 1.02453 & \\
10 & $3.1 \%$ & $0.6 \%$ & $0.5 \%$ & $0.5 \%$ & $0.5 \%$ & $94.8 \%$ & 1.11109 & \\
11 & $18.6 \%$ & $80.4 \%$ & $0.3 \%$ & $0.3 \%$ & $0.3 \%$ & $0.1 \%$ & 1.46821 & \\
12 & $51.2 \%$ & $1.8 \%$ & $44.7 \%$ & $0.6 \%$ & $0.6 \%$ & $1.1 \%$ & 2.16130 & \\
13 & $51.2 \%$ & $1.8 \%$ & $0.6 \%$ & $44.7 \%$ & $0.6 \%$ & $1.1 \%$ & 2.16225 & \\
14 & $51.2 \%$ & $1.8 \%$ & $0.6 \%$ & $0.6 \%$ & $44.7 \%$ & $1.1 \%$ & 2.16067 & \\
\hline \hline
\end{tabular}


S1.5.16 IRC Step 16

\begin{tabular}{|c|c|c|}
\hline \multicolumn{3}{|c|}{ ב1-center FNO analysis, $\varepsilon=0.85000$} \\
\hline $\mathrm{MO}$ & $\lambda$ & Atoms \\
\hline 1 & 1.00183 & $\mathrm{C}_{1}$ \\
\hline 2 & 1.00020 & $\mathrm{~F}_{2}$ \\
\hline 3 & 0.99843 & $\mathrm{~F}_{2}$ \\
\hline 4 & 0.98304 & $\mathrm{~F}_{2}$ \\
\hline 5 & 0.98303 & $\mathrm{~F}_{2}$ \\
\hline 6 & 1.00008 & $\mathrm{~F}_{6}$ \\
\hline 7 & 0.99903 & $\mathrm{~F}_{6}$ \\
\hline 8 & 0.98777 & $\mathrm{~F}_{6}$ \\
\hline 9 & 0.98776 & $\mathrm{~F}_{6}$ \\
\hline 10 & 0.94292 & $\mathrm{~F}_{6}$ \\
\hline \multicolumn{3}{|c|}{ 2-center FNO analysis, $\varepsilon=0.85000$} \\
\hline $\mathrm{MO}$ & $\lambda$ & Atoms \\
\hline 11 & 0.98966 & $\mathrm{C}_{1}-\mathrm{F}_{2}$ \\
\hline 12 & 0.95978 & $\mathrm{C}_{1}-\mathrm{H}_{3}$ \\
\hline 13 & 0.95976 & $\mathrm{C}_{1}-\mathrm{H}_{4}$ \\
\hline 14 & 0.95986 & $\mathrm{C}_{1}-\mathrm{H}_{5}$ \\
\hline
\end{tabular}

\begin{tabular}{crrrrrrrr}
\hline \hline \multicolumn{2}{l}{ Localization and atoms expanded by each FNO } & & & \\
\hline FNO & Loc $_{1}$ & Loc $_{2}$ & Loc $_{3}$ & Loc $_{4}$ & Loc $_{5}$ & Loc $_{6}$ & Loc $_{7}$ & $n_{\text {eff }}$ \\
\hline 1 & $100.2 \%$ & $0.0 \%$ & $0.0 \%$ & $0.0 \%$ & $0.0 \%$ & $0.0 \%$ & 0.99634 & \\
2 & $0.0 \%$ & $100.0 \%$ & $0.0 \%$ & $0.0 \%$ & $0.0 \%$ & $0.0 \%$ & 0.99960 & \\
3 & $0.1 \%$ & $99.8 \%$ & $0.0 \%$ & $0.0 \%$ & $0.0 \%$ & $0.0 \%$ & 1.00314 & \\
4 & $1.1 \%$ & $98.3 \%$ & $0.2 \%$ & $0.0 \%$ & $0.2 \%$ & $0.0 \%$ & 1.03464 & \\
5 & $1.1 \%$ & $98.3 \%$ & $0.1 \%$ & $0.3 \%$ & $0.1 \%$ & $0.0 \%$ & 1.03467 & \\
6 & $0.0 \%$ & $0.0 \%$ & $0.0 \%$ & $0.0 \%$ & $0.0 \%$ & $100.0 \%$ & 0.99984 & \\
7 & $0.0 \%$ & $0.0 \%$ & $0.0 \%$ & $0.0 \%$ & $0.0 \%$ & $99.9 \%$ & 1.00194 & \\
8 & $0.4 \%$ & $0.0 \%$ & $0.4 \%$ & $0.1 \%$ & $0.3 \%$ & $98.8 \%$ & 1.02486 & \\
9 & $0.4 \%$ & $0.0 \%$ & $0.1 \%$ & $0.4 \%$ & $0.3 \%$ & $98.8 \%$ & 1.02490 & \\
10 & $3.6 \%$ & $0.7 \%$ & $0.5 \%$ & $0.5 \%$ & $0.5 \%$ & $94.3 \%$ & 1.12299 & \\
11 & $18.5 \%$ & $80.5 \%$ & $0.3 \%$ & $0.3 \%$ & $0.3 \%$ & $0.1 \%$ & 1.46691 & \\
12 & $51.4 \%$ & $1.7 \%$ & $44.6 \%$ & $0.6 \%$ & $0.6 \%$ & $1.1 \%$ & 2.15822 & \\
13 & $51.3 \%$ & $1.7 \%$ & $0.6 \%$ & $44.6 \%$ & $0.6 \%$ & $1.1 \%$ & 2.15832 & \\
14 & $51.4 \%$ & $1.7 \%$ & $0.6 \%$ & $0.6 \%$ & $44.6 \%$ & $1.1 \%$ & 2.15786 & \\
\hline \hline
\end{tabular}

S1.5.17 IRC Step 17

\begin{tabular}{ccc}
\hline \hline \multicolumn{3}{c}{ 1-center FNO analysis, $\varepsilon=0.85000$} \\
\hline $\mathrm{MO}$ & $\lambda$ & Atoms \\
\hline 1 & 1.00178 & $\mathrm{C}_{1}$ \\
2 & 1.00019 & $\mathrm{~F}_{2}$ \\
3 & 0.99842 & $\mathrm{~F}_{2}$ \\
4 & 0.98359 & $\mathrm{~F}_{2}$ \\
5 & 0.98356 & $\mathrm{~F}_{2}$ \\
6 & 1.00008 & $\mathrm{~F}_{6}$ \\
7 & 0.99895 & $\mathrm{~F}_{6}$ \\
8 & 0.98761 & $\mathrm{~F}_{6}$ \\
9 & 0.98760 & $\mathrm{~F}_{6}$ \\
10 & 0.93691 & $\mathrm{~F}_{6}$ \\
\hline 2 -center $\mathrm{FNO}$ analysis, $\varepsilon=0.85000$ \\
\hline MO & $\lambda$ & Atoms \\
\hline 11 & 0.98918 & $\mathrm{C}_{1}-\mathrm{F}_{2}$ \\
12 & 0.96030 & $\mathrm{C}_{1}-\mathrm{H}_{3}$ \\
13 & 0.96039 & $\mathrm{C}_{1}-\mathrm{H}_{4}$ \\
14 & 0.96050 & $\mathrm{C}_{1}-\mathrm{H}_{5}$ \\
\hline \hline
\end{tabular}




\begin{tabular}{crrrrrrrr}
\hline \hline \multicolumn{2}{l}{ Localization and atoms expanded by each FNO } \\
\hline FNO & Loc $_{1}$ & \multicolumn{1}{l}{ Loc $_{2}$} & Loc $_{3}$ & Loc $_{4}$ & Loc $_{5}$ & Loc $_{6}$ & Loc $_{7}$ & $n_{\text {eff }}$ \\
\hline 1 & $100.2 \%$ & $0.0 \%$ & $0.0 \%$ & $0.0 \%$ & $0.0 \%$ & $0.0 \%$ & 0.99645 & \\
2 & $0.0 \%$ & $100.0 \%$ & $0.0 \%$ & $0.0 \%$ & $0.0 \%$ & $0.0 \%$ & 0.99961 & \\
3 & $0.1 \%$ & $99.8 \%$ & $0.0 \%$ & $0.0 \%$ & $0.0 \%$ & $0.0 \%$ & 1.00318 & \\
4 & $1.1 \%$ & $98.4 \%$ & $0.0 \%$ & $0.2 \%$ & $0.3 \%$ & $0.0 \%$ & 1.03351 & \\
5 & $1.1 \%$ & $98.4 \%$ & $0.3 \%$ & $0.1 \%$ & $0.1 \%$ & $0.0 \%$ & 1.03356 & \\
6 & $0.0 \%$ & $0.0 \%$ & $0.0 \%$ & $0.0 \%$ & $0.0 \%$ & $100.0 \%$ & 0.99983 & \\
7 & $0.0 \%$ & $0.0 \%$ & $0.0 \%$ & $0.0 \%$ & $0.0 \%$ & $99.9 \%$ & 1.00210 & \\
8 & $0.4 \%$ & $0.0 \%$ & $0.2 \%$ & $0.4 \%$ & $0.2 \%$ & $98.8 \%$ & 1.02521 & \\
9 & $0.4 \%$ & $0.0 \%$ & $0.4 \%$ & $0.1 \%$ & $0.4 \%$ & $98.8 \%$ & 1.02523 & \\
10 & $4.1 \%$ & $0.8 \%$ & $0.5 \%$ & $0.5 \%$ & $0.5 \%$ & $93.7 \%$ & 1.13690 & \\
11 & $18.3 \%$ & $80.6 \%$ & $0.3 \%$ & $0.3 \%$ & $0.3 \%$ & $0.1 \%$ & 1.46263 & \\
12 & $51.5 \%$ & $1.7 \%$ & $44.6 \%$ & $0.6 \%$ & $0.6 \%$ & $1.1 \%$ & 2.15540 & \\
13 & $51.5 \%$ & $1.7 \%$ & $0.6 \%$ & $44.6 \%$ & $0.6 \%$ & $1.1 \%$ & 2.15496 & \\
14 & $51.5 \%$ & $1.7 \%$ & $0.6 \%$ & $0.6 \%$ & $44.6 \%$ & $1.1 \%$ & 2.15447 & \\
\hline \hline
\end{tabular}

\section{S1.5.18 IRC Step 18}

\begin{tabular}{ccc}
\hline \hline \multicolumn{3}{c}{ 1-center FNO analysis, $\varepsilon=0.85000$} \\
\hline MO & $\lambda$ & Atoms \\
\hline 1 & 1.00171 & $\mathrm{C}_{1}$ \\
2 & 1.00019 & $\mathrm{~F}_{2}$ \\
3 & 0.99841 & $\mathrm{~F}_{2}$ \\
4 & 0.98411 & $\mathrm{~F}_{2}$ \\
5 & 0.98408 & $\mathrm{~F}_{2}$ \\
6 & 1.00009 & $\mathrm{~F}_{6}$ \\
7 & 0.99887 & $\mathrm{~F}_{6}$ \\
8 & 0.98749 & $\mathrm{~F}_{6}$ \\
9 & 0.98749 & $\mathrm{~F}_{6}$ \\
10 & 0.92997 & $\mathrm{~F}_{6}$ \\
\hline 2 -center FNO analysis, $\varepsilon=0.85000$ \\
\hline MO & $\lambda$ & Atoms $_{1}$ \\
\hline 11 & 0.98912 & $\mathrm{C}_{1}-\mathrm{F}_{2}$ \\
12 & 0.96106 & $\mathrm{C}_{1}-\mathrm{H}_{3}$ \\
13 & 0.96101 & $\mathrm{C}_{1}-\mathrm{H}_{4}$ \\
14 & 0.96113 & $\mathrm{C}_{1}-\mathrm{H}_{5}$ \\
\hline \hline
\end{tabular}

\begin{tabular}{crrrrrrrr}
\hline \multicolumn{1}{l}{ Localization and atoms expanded by each FNO } & & & \\
\hline \hline FNO & Loc $_{1}$ & Loc $_{2}$ & Loc $_{3}$ & Loc $_{4}$ & Loc $_{5}$ & Loc $_{6}$ & Loc $_{7}$ & $n_{\text {eff }}$ \\
\hline 1 & $100.2 \%$ & $0.0 \%$ & $0.0 \%$ & $0.0 \%$ & $0.0 \%$ & $0.0 \%$ & 0.99659 & \\
2 & $0.0 \%$ & $100.0 \%$ & $0.0 \%$ & $0.0 \%$ & $0.0 \%$ & $0.0 \%$ & 0.99962 & \\
3 & $0.1 \%$ & $99.8 \%$ & $0.0 \%$ & $0.0 \%$ & $0.0 \%$ & $0.0 \%$ & 1.00319 & \\
4 & $1.0 \%$ & $98.4 \%$ & $0.1 \%$ & $0.1 \%$ & $0.3 \%$ & $0.0 \%$ & 1.03243 & \\
5 & $1.0 \%$ & $98.4 \%$ & $0.3 \%$ & $0.2 \%$ & $0.0 \%$ & $0.0 \%$ & 1.03249 & \\
6 & $0.0 \%$ & $0.0 \%$ & $0.0 \%$ & $0.0 \%$ & $0.0 \%$ & $100.0 \%$ & 0.99982 & \\
7 & $0.1 \%$ & $0.0 \%$ & $0.0 \%$ & $0.0 \%$ & $0.0 \%$ & $99.9 \%$ & 1.00226 & \\
8 & $0.4 \%$ & $0.0 \%$ & $0.4 \%$ & $0.3 \%$ & $0.1 \%$ & $98.7 \%$ & 1.02544 & \\
9 & $0.4 \%$ & $0.0 \%$ & $0.1 \%$ & $0.3 \%$ & $0.4 \%$ & $98.7 \%$ & 1.02545 & \\
10 & $4.7 \%$ & $0.9 \%$ & $0.5 \%$ & $0.5 \%$ & $0.5 \%$ & $93.0 \%$ & 1.15320 & \\
11 & $18.0 \%$ & $80.9 \%$ & $0.3 \%$ & $0.3 \%$ & $0.3 \%$ & $0.1 \%$ & 1.45460 & \\
12 & $51.6 \%$ & $1.6 \%$ & $44.5 \%$ & $0.5 \%$ & $0.5 \%$ & $1.2 \%$ & 2.15155 & \\
13 & $51.6 \%$ & $1.6 \%$ & $0.5 \%$ & $44.5 \%$ & $0.5 \%$ & $1.2 \%$ & 2.15189 & \\
14 & $51.6 \%$ & $1.6 \%$ & $0.5 \%$ & $0.5 \%$ & $44.5 \%$ & $1.2 \%$ & 2.15127 & \\
\hline \hline
\end{tabular}


S1.5.19 IRC Step 19

\begin{tabular}{ccc}
\hline \hline \multicolumn{3}{c}{ 1-center FNO analysis, $\varepsilon=0.85000$} \\
\hline MO & $\lambda$ & Atoms \\
\hline 1 & 1.00163 & $\mathrm{C}_{1}$ \\
2 & 1.00018 & $\mathrm{~F}_{2}$ \\
3 & 0.99840 & $\mathrm{~F}_{2}$ \\
4 & 0.98455 & $\mathrm{~F}_{2}$ \\
5 & 0.98454 & $\mathrm{~F}_{2}$ \\
6 & 1.00010 & $\mathrm{~F}_{6}$ \\
7 & 0.99879 & $\mathrm{~F}_{6}$ \\
8 & 0.98740 & $\mathrm{~F}_{6}$ \\
9 & 0.98737 & $\mathrm{~F}_{6}$ \\
10 & 0.92226 & $\mathrm{~F}_{6}$ \\
\hline 2 -center FNO analysis, $\varepsilon=0.85000$ \\
\hline MO & $\lambda$ & Atoms $^{2}$ \\
\hline 11 & 0.98838 & $\mathrm{C}_{1}-\mathrm{F}_{2}$ \\
12 & 0.96119 & $\mathrm{C}_{1}-\mathrm{H}_{3}$ \\
13 & 0.96116 & $\mathrm{C}_{1}-\mathrm{H}_{4}$ \\
14 & 0.96110 & $\mathrm{C}_{1}-\mathrm{H}_{5}$ \\
\hline \hline
\end{tabular}

\begin{tabular}{crrrrrrrr}
\hline \multicolumn{2}{l}{ Localization and atoms expanded by each FNO } & & & \\
\hline FNO & $\mathrm{Loc}_{1}$ & $\mathrm{Loc}_{2}$ & $\mathrm{Loc}_{3}$ & $\mathrm{Loc}_{4}$ & $\mathrm{Loc}_{5}$ & $\mathrm{Loc}_{6}$ & $\mathrm{Loc}_{7}$ & $n_{\mathrm{eff}}$ \\
\hline 1 & $100.2 \%$ & $0.0 \%$ & $0.0 \%$ & $0.0 \%$ & $0.0 \%$ & $0.0 \%$ & 0.99675 & \\
2 & $0.0 \%$ & $100.0 \%$ & $0.0 \%$ & $0.0 \%$ & $0.0 \%$ & $0.0 \%$ & 0.99964 & \\
3 & $0.1 \%$ & $99.8 \%$ & $0.0 \%$ & $0.0 \%$ & $0.0 \%$ & $0.0 \%$ & 1.00322 & \\
4 & $1.0 \%$ & $98.5 \%$ & $0.2 \%$ & $0.2 \%$ & $0.0 \%$ & $0.0 \%$ & 1.03152 & \\
5 & $1.0 \%$ & $98.5 \%$ & $0.1 \%$ & $0.1 \%$ & $0.3 \%$ & $0.0 \%$ & 1.03155 & \\
6 & $0.0 \%$ & $0.0 \%$ & $0.0 \%$ & $0.0 \%$ & $0.0 \%$ & $100.0 \%$ & 0.99981 & \\
7 & $0.1 \%$ & $0.0 \%$ & $0.0 \%$ & $0.0 \%$ & $0.0 \%$ & $99.9 \%$ & 1.00242 & \\
8 & $0.5 \%$ & $0.0 \%$ & $0.3 \%$ & $0.4 \%$ & $0.1 \%$ & $98.7 \%$ & 1.02564 & \\
9 & $0.5 \%$ & $0.0 \%$ & $0.2 \%$ & $0.1 \%$ & $0.4 \%$ & $98.7 \%$ & 1.02569 & \\
10 & $5.3 \%$ & $0.9 \%$ & $0.5 \%$ & $0.5 \%$ & $0.5 \%$ & $92.2 \%$ & 1.17157 & \\
11 & $17.6 \%$ & $81.3 \%$ & $0.3 \%$ & $0.3 \%$ & $0.3 \%$ & $0.2 \%$ & 1.44640 & \\
12 & $51.6 \%$ & $1.6 \%$ & $44.5 \%$ & $0.5 \%$ & $0.5 \%$ & $1.2 \%$ & 2.15082 & \\
13 & $51.6 \%$ & $1.6 \%$ & $0.5 \%$ & $44.5 \%$ & $0.5 \%$ & $1.2 \%$ & 2.15086 & \\
14 & $51.6 \%$ & $1.6 \%$ & $0.5 \%$ & $0.5 \%$ & $44.5 \%$ & $1.2 \%$ & 2.15119 & \\
\hline \hline
\end{tabular}

\section{S1.5.20 IRC Step 20}

\begin{tabular}{|c|c|c|}
\hline \multicolumn{3}{|c|}{ 1-center FNO analysis, $\varepsilon=0.85000$} \\
\hline $\mathrm{MO}$ & $\lambda$ & Atoms \\
\hline 1 & 1.00155 & $\mathrm{C}_{1}$ \\
\hline 2 & 1.00018 & $\mathrm{~F}_{2}$ \\
\hline 3 & 0.99839 & $\mathrm{~F}_{2}$ \\
\hline 4 & 0.98499 & $\mathrm{~F}_{2}$ \\
\hline 5 & 0.98497 & $\mathrm{~F}_{2}$ \\
\hline 6 & 1.00010 & $\mathrm{~F}_{6}$ \\
\hline 7 & 0.99872 & $\mathrm{~F}_{6}$ \\
\hline 8 & 0.98729 & $\mathrm{~F}_{6}$ \\
\hline 9 & 0.98728 & $\mathrm{~F}_{6}$ \\
\hline 10 & 0.91398 & $\mathrm{~F}_{6}$ \\
\hline \multicolumn{3}{|c|}{2 -center FNO analysis, $\varepsilon=0.85000$} \\
\hline $\mathrm{MO}$ & $\lambda$ & Atoms \\
\hline 11 & 0.98727 & $\mathrm{C}_{1}-\mathrm{F}_{2}$ \\
\hline 12 & 0.96166 & $\mathrm{C}_{1}-\mathrm{H}_{3}$ \\
\hline 13 & 0.96150 & $\mathrm{C}_{1}-\mathrm{H}_{4}$ \\
\hline 14 & 0.96172 & $\mathrm{C}_{1}-\mathrm{H}_{5}$ \\
\hline
\end{tabular}




\begin{tabular}{crrrrrrrr}
\hline \hline \multicolumn{2}{l}{ Localization and atoms expanded by each FNO } \\
\hline FNO & Loc $_{1}$ & Loc $_{2}$ & Loc $_{3}$ & Loc $_{4}$ & Loc $_{5}$ & Loc $_{6}$ & Loc $_{7}$ & $n_{\text {eff }}$ \\
\hline 1 & $100.2 \%$ & $0.0 \%$ & $0.0 \%$ & $0.0 \%$ & $0.0 \%$ & $0.0 \%$ & 0.99692 & \\
2 & $0.0 \%$ & $100.0 \%$ & $0.0 \%$ & $0.0 \%$ & $0.0 \%$ & $0.0 \%$ & 0.99965 & \\
3 & $0.1 \%$ & $99.8 \%$ & $0.0 \%$ & $0.0 \%$ & $0.0 \%$ & $0.0 \%$ & 1.00323 & \\
4 & $0.9 \%$ & $98.5 \%$ & $0.0 \%$ & $0.2 \%$ & $0.2 \%$ & $0.0 \%$ & 1.03061 & \\
5 & $0.9 \%$ & $98.5 \%$ & $0.3 \%$ & $0.1 \%$ & $0.1 \%$ & $0.0 \%$ & 1.03065 & \\
6 & $0.0 \%$ & $0.0 \%$ & $0.0 \%$ & $0.0 \%$ & $0.0 \%$ & $100.0 \%$ & 0.99980 & \\
7 & $0.1 \%$ & $0.0 \%$ & $0.0 \%$ & $0.0 \%$ & $0.0 \%$ & $99.9 \%$ & 1.00256 & \\
8 & $0.5 \%$ & $0.0 \%$ & $0.2 \%$ & $0.1 \%$ & $0.4 \%$ & $98.7 \%$ & 1.02586 & \\
9 & $0.5 \%$ & $0.0 \%$ & $0.3 \%$ & $0.4 \%$ & $0.1 \%$ & $98.7 \%$ & 1.02588 & \\
10 & $6.0 \%$ & $1.0 \%$ & $0.5 \%$ & $0.5 \%$ & $0.5 \%$ & $91.4 \%$ & 1.19161 & \\
11 & $17.0 \%$ & $81.7 \%$ & $0.4 \%$ & $0.4 \%$ & $0.4 \%$ & $0.2 \%$ & 1.43612 & \\
12 & $51.7 \%$ & $1.6 \%$ & $44.5 \%$ & $0.5 \%$ & $0.5 \%$ & $1.2 \%$ & 2.14859 & \\
13 & $51.7 \%$ & $1.6 \%$ & $0.5 \%$ & $44.5 \%$ & $0.5 \%$ & $1.2 \%$ & 2.14930 & \\
14 & $51.7 \%$ & $1.6 \%$ & $0.5 \%$ & $0.5 \%$ & $44.5 \%$ & $1.2 \%$ & 2.14841 & \\
\hline \hline
\end{tabular}

\section{S1.5.21 IRC Step 21}

\begin{tabular}{ccc}
\hline \hline \multicolumn{3}{c}{ 1-center FNO analysis, $\varepsilon=0.85000$} \\
\hline MO & $\lambda$ & Atoms \\
\hline 1 & 1.00146 & $\mathrm{C}_{1}$ \\
2 & 1.00017 & $\mathrm{~F}_{2}$ \\
3 & 0.99839 & $\mathrm{~F}_{2}$ \\
4 & 0.98539 & $\mathrm{~F}_{2}$ \\
5 & 0.98536 & $\mathrm{~F}_{2}$ \\
6 & 1.00011 & $\mathrm{~F}_{6}$ \\
7 & 0.99865 & $\mathrm{~F}_{6}$ \\
8 & 0.98720 & $\mathrm{~F}_{6}$ \\
9 & 0.98718 & $\mathrm{~F}_{6}$ \\
10 & 0.90506 & $\mathrm{~F}_{6}$ \\
\hline 2 -center FNO analysis, $\varepsilon=0.85000$ \\
\hline MO & $\lambda$ & Atoms \\
\hline 11 & 0.98654 & $\mathrm{C}_{1}-\mathrm{F}_{2}$ \\
12 & 0.96175 & $\mathrm{C}_{1}-\mathrm{H}_{3}$ \\
13 & 0.96176 & $\mathrm{C}_{1}-\mathrm{H}_{4}$ \\
14 & 0.96233 & $\mathrm{C}_{1}-\mathrm{H}_{5}$ \\
\hline \hline
\end{tabular}

\begin{tabular}{crrrrrrrr}
\hline \hline \multicolumn{2}{l}{ Localization and atoms expanded by each FNO } & & & \\
\hline FNO & Loc $_{1}$ & $\mathrm{Loc}_{2}$ & $\mathrm{Loc}_{3}$ & Loc $_{4}$ & $\mathrm{Loc}_{5}$ & $\mathrm{Loc}_{6}$ & $\mathrm{Loc}_{7}$ & $n_{\text {eff }}$ \\
\hline 1 & $100.1 \%$ & $0.0 \%$ & $0.0 \%$ & $0.0 \%$ & $0.0 \%$ & $0.0 \%$ & 0.99708 & \\
2 & $0.0 \%$ & $100.0 \%$ & $0.0 \%$ & $0.0 \%$ & $0.0 \%$ & $0.0 \%$ & 0.99966 & \\
3 & $0.1 \%$ & $99.8 \%$ & $0.0 \%$ & $0.0 \%$ & $0.0 \%$ & $0.0 \%$ & 1.00322 & \\
4 & $0.9 \%$ & $98.5 \%$ & $0.1 \%$ & $0.1 \%$ & $0.3 \%$ & $0.0 \%$ & 1.02978 & \\
5 & $0.9 \%$ & $98.5 \%$ & $0.2 \%$ & $0.2 \%$ & $0.0 \%$ & $0.0 \%$ & 1.02983 & \\
6 & $0.0 \%$ & $0.0 \%$ & $0.0 \%$ & $0.0 \%$ & $0.0 \%$ & $100.0 \%$ & 0.99978 & \\
7 & $0.1 \%$ & $0.0 \%$ & $0.0 \%$ & $0.0 \%$ & $0.0 \%$ & $99.9 \%$ & 1.00270 & \\
8 & $0.5 \%$ & $0.0 \%$ & $0.3 \%$ & $0.1 \%$ & $0.4 \%$ & $98.7 \%$ & 1.02605 & \\
9 & $0.5 \%$ & $0.0 \%$ & $0.2 \%$ & $0.4 \%$ & $0.1 \%$ & $98.7 \%$ & 1.02609 & \\
10 & $6.8 \%$ & $1.1 \%$ & $0.5 \%$ & $0.5 \%$ & $0.5 \%$ & $90.5 \%$ & 1.21356 & \\
11 & $16.4 \%$ & $82.2 \%$ & $0.4 \%$ & $0.4 \%$ & $0.4 \%$ & $0.2 \%$ & 1.42244 & \\
12 & $51.7 \%$ & $1.5 \%$ & $44.5 \%$ & $0.5 \%$ & $0.5 \%$ & $1.3 \%$ & 2.14813 & \\
13 & $51.7 \%$ & $1.5 \%$ & $0.5 \%$ & $44.5 \%$ & $0.5 \%$ & $1.3 \%$ & 2.14804 & \\
14 & $51.7 \%$ & $1.5 \%$ & $0.5 \%$ & $0.5 \%$ & $44.5 \%$ & $1.3 \%$ & 2.14538 & \\
\hline \hline
\end{tabular}


S1.5.22 IRC Step 22

\begin{tabular}{ccc}
\hline \hline \multicolumn{3}{c}{ 1-center FNO analysis, $\varepsilon=0.85000$} \\
\hline MO & $\lambda$ & Atoms \\
\hline 1 & 1.00146 & $\mathrm{C}_{1}$ \\
2 & 1.00016 & $\mathrm{~F}_{2}$ \\
3 & 0.99840 & $\mathrm{~F}_{2}$ \\
4 & 0.98572 & $\mathrm{~F}_{2}$ \\
5 & 0.98570 & $\mathrm{~F}_{2}$ \\
6 & 1.00011 & $\mathrm{~F}_{6}$ \\
7 & 0.99860 & $\mathrm{~F}_{6}$ \\
8 & 0.98707 & $\mathrm{~F}_{6}$ \\
9 & 0.98706 & $\mathrm{~F}_{6}$ \\
10 & 0.89611 & $\mathrm{~F}_{6}$ \\
\hline 2 -center FNO analysis, $=0.85000$ \\
\hline MO & $\lambda$ & Atoms $^{2}$ \\
\hline 11 & 0.98571 & $\mathrm{C}_{1}-\mathrm{F}_{2}$ \\
12 & 0.96207 & $\mathrm{C}_{1}-\mathrm{H}_{3}$ \\
13 & 0.96196 & $\mathrm{C}_{1}-\mathrm{H}_{4}$ \\
14 & 0.96193 & $\mathrm{C}_{1}-\mathrm{H}_{5}$ \\
\hline \hline
\end{tabular}

\begin{tabular}{crrrrrrrr}
\hline \multicolumn{2}{l}{ Localization and atoms expanded by each FNO } & & & \\
\hline FNO & $\mathrm{Loc}_{1}$ & $\mathrm{Loc}_{2}$ & $\mathrm{Loc}_{3}$ & $\mathrm{Loc}_{4}$ & $\mathrm{Loc}_{5}$ & $\mathrm{Loc}_{6}$ & $\mathrm{Loc}_{7}$ & $n_{\mathrm{eff}}$ \\
\hline 1 & $100.1 \%$ & $0.0 \%$ & $0.0 \%$ & $0.0 \%$ & $0.0 \%$ & $0.0 \%$ & 0.99708 & \\
2 & $0.0 \%$ & $100.0 \%$ & $0.0 \%$ & $0.0 \%$ & $0.0 \%$ & $0.0 \%$ & 0.99968 & \\
3 & $0.1 \%$ & $99.8 \%$ & $0.0 \%$ & $0.0 \%$ & $0.0 \%$ & $0.0 \%$ & 1.00321 & \\
4 & $0.8 \%$ & $98.6 \%$ & $0.1 \%$ & $0.3 \%$ & $0.1 \%$ & $0.0 \%$ & 1.02911 & \\
5 & $0.8 \%$ & $98.6 \%$ & $0.2 \%$ & $0.1 \%$ & $0.3 \%$ & $0.0 \%$ & 1.02913 & \\
6 & $0.0 \%$ & $0.0 \%$ & $0.0 \%$ & $0.0 \%$ & $0.0 \%$ & $100.0 \%$ & 0.99977 & \\
7 & $0.1 \%$ & $0.0 \%$ & $0.0 \%$ & $0.0 \%$ & $0.0 \%$ & $99.9 \%$ & 1.00281 & \\
8 & $0.6 \%$ & $0.0 \%$ & $0.2 \%$ & $0.4 \%$ & $0.2 \%$ & $98.7 \%$ & 1.02631 & \\
9 & $0.6 \%$ & $0.0 \%$ & $0.3 \%$ & $0.1 \%$ & $0.3 \%$ & $98.7 \%$ & 1.02633 & \\
10 & $7.6 \%$ & $1.2 \%$ & $0.5 \%$ & $0.5 \%$ & $0.5 \%$ & $89.6 \%$ & 1.23598 & \\
11 & $15.7 \%$ & $82.8 \%$ & $0.4 \%$ & $0.4 \%$ & $0.4 \%$ & $0.2 \%$ & 1.40653 & \\
12 & $51.7 \%$ & $1.5 \%$ & $44.5 \%$ & $0.5 \%$ & $0.5 \%$ & $1.3 \%$ & 2.14680 & \\
13 & $51.7 \%$ & $1.5 \%$ & $0.5 \%$ & $44.5 \%$ & $0.5 \%$ & $1.3 \%$ & 2.14720 & \\
14 & $51.7 \%$ & $1.5 \%$ & $0.5 \%$ & $0.5 \%$ & $44.5 \%$ & $1.3 \%$ & 2.14737 & \\
\hline \hline
\end{tabular}

\section{S1.5.23 IRC Step 23}

\begin{tabular}{ccc}
\hline \hline \multicolumn{3}{c}{ 1-center FNO analysis, $\varepsilon=0.85000$} \\
\hline MO & $\lambda$ & Atoms \\
\hline 1 & 1.00147 & $\mathrm{C}_{1}$ \\
2 & 1.00015 & $\mathrm{~F}_{2}$ \\
3 & 0.99842 & $\mathrm{~F}_{2}$ \\
4 & 0.98602 & $\mathrm{~F}_{2}$ \\
5 & 0.98601 & $\mathrm{~F}_{2}$ \\
6 & 0.85475 & $\mathrm{~F}_{2}$ \\
7 & 1.00012 & $\mathrm{~F}_{6}$ \\
8 & 0.99855 & $\mathrm{~F}_{6}$ \\
9 & 0.98691 & $\mathrm{~F}_{6}$ \\
10 & 0.98691 & $\mathrm{~F}_{6}$ \\
11 & 0.88734 & $\mathrm{~F}_{6}$ \\
\hline 2 -center $\mathrm{FNO}$ analysis, $\varepsilon=0.85000$ \\
\hline MO & $\lambda$ & Atoms $^{2}$ \\
\hline 12 & 0.96234 & $\mathrm{C}_{1}-\mathrm{H}_{3}$ \\
13 & 0.96207 & $\mathrm{C}_{1}-\mathrm{H}_{4}$ \\
14 & 0.96190 & $\mathrm{C}_{1}-\mathrm{H}_{5}$ \\
\hline \hline
\end{tabular}




\begin{tabular}{crrrrrrrr}
\hline \hline \multicolumn{2}{l}{ Localization and atoms expanded by each FNO } \\
\hline FNO & Loc $_{1}$ & Loc $_{2}$ & Loc $_{3}$ & Loc $_{4}$ & Loc $_{5}$ & Loc $_{6}$ & Loc $_{7}$ & $n_{\text {eff }}$ \\
\hline 1 & $100.1 \%$ & $0.0 \%$ & $0.0 \%$ & $0.0 \%$ & $0.0 \%$ & $0.0 \%$ & 0.99708 & \\
2 & $0.0 \%$ & $100.0 \%$ & $0.0 \%$ & $0.0 \%$ & $0.0 \%$ & $0.0 \%$ & 0.99970 & \\
3 & $0.1 \%$ & $99.8 \%$ & $0.0 \%$ & $0.0 \%$ & $0.0 \%$ & $0.0 \%$ & 1.00317 & \\
4 & $0.8 \%$ & $98.6 \%$ & $0.3 \%$ & $0.2 \%$ & $0.1 \%$ & $0.0 \%$ & 1.02848 & \\
5 & $0.8 \%$ & $98.6 \%$ & $0.1 \%$ & $0.1 \%$ & $0.3 \%$ & $0.0 \%$ & 1.02849 & \\
6 & $11.8 \%$ & $85.5 \%$ & $0.5 \%$ & $0.5 \%$ & $0.5 \%$ & $1.2 \%$ & 1.34290 & \\
7 & $0.0 \%$ & $0.0 \%$ & $0.0 \%$ & $0.0 \%$ & $0.0 \%$ & $100.0 \%$ & 0.99976 & \\
8 & $0.1 \%$ & $0.0 \%$ & $0.0 \%$ & $0.0 \%$ & $0.0 \%$ & $99.9 \%$ & 1.00291 & \\
9 & $0.6 \%$ & $0.0 \%$ & $0.3 \%$ & $0.3 \%$ & $0.1 \%$ & $98.7 \%$ & 1.02664 & \\
10 & $0.6 \%$ & $0.0 \%$ & $0.2 \%$ & $0.1 \%$ & $0.4 \%$ & $98.7 \%$ & 1.02666 & \\
11 & $8.5 \%$ & $1.2 \%$ & $0.5 \%$ & $0.5 \%$ & $0.5 \%$ & $88.7 \%$ & 1.25821 & \\
12 & $51.7 \%$ & $1.4 \%$ & $44.5 \%$ & $0.5 \%$ & $0.5 \%$ & $1.3 \%$ & 2.14572 & \\
13 & $51.7 \%$ & $1.4 \%$ & $0.5 \%$ & $44.5 \%$ & $0.5 \%$ & $1.3 \%$ & 2.14684 & \\
14 & $51.7 \%$ & $1.4 \%$ & $0.5 \%$ & $0.5 \%$ & $44.5 \%$ & $1.3 \%$ & 2.14771 & \\
\hline \hline
\end{tabular}

S1.5.24 IRC Step 24

\begin{tabular}{ccc}
\hline \multicolumn{3}{c}{ 1-center FNO } \\
\hline $\mathrm{MO}$ & $\lambda$ & analysis, $\varepsilon=0.85000$ \\
\hline 1 & 1.00147 & $\mathrm{C}_{1}$ \\
2 & 1.00014 & $\mathrm{~F}_{2}$ \\
3 & 0.99846 & $\mathrm{~F}_{2}$ \\
4 & 0.98653 & $\mathrm{~F}_{2}$ \\
5 & 0.98653 & $\mathrm{~F}_{2}$ \\
6 & 0.87006 & $\mathrm{~F}_{2}$ \\
7 & 1.00014 & $\mathrm{~F}_{6}$ \\
8 & 0.99846 & $\mathrm{~F}_{6}$ \\
9 & 0.98653 & $\mathrm{~F}_{6}$ \\
10 & 0.98653 & $\mathrm{~F}_{6}$ \\
11 & 0.87006 & $\mathrm{~F}_{6}$ \\
\hline 2 -center FNO analysis, $\varepsilon=0.85000$ \\
\hline MO & $\lambda$ & Atoms \\
\hline 12 & 0.96200 & $\mathrm{C}_{1}-\mathrm{H}_{3}$ \\
13 & 0.96207 & $\mathrm{C}_{1}-\mathrm{H}_{4}$ \\
14 & 0.96170 & $\mathrm{C}_{1}-\mathrm{H}_{5}$ \\
\hline \hline
\end{tabular}

\begin{tabular}{crrrrrrrr}
\hline \hline \multicolumn{2}{l}{ Localization and atoms expanded by each FNO } & & & \\
\hline FNO & Loc $_{1}$ & Loc $_{2}$ & Loc $_{3}$ & Loc $_{4}$ & Loc $_{5}$ & Loc $_{6}$ & Loc $_{7}$ & $n_{\text {eff }}$ \\
\hline 1 & $100.1 \%$ & $0.0 \%$ & $0.0 \%$ & $0.0 \%$ & $0.0 \%$ & $0.0 \%$ & 0.99707 & \\
2 & $0.0 \%$ & $100.0 \%$ & $0.0 \%$ & $0.0 \%$ & $0.0 \%$ & $0.0 \%$ & 0.99973 & \\
3 & $0.1 \%$ & $99.8 \%$ & $0.0 \%$ & $0.0 \%$ & $0.0 \%$ & $0.0 \%$ & 1.00308 & \\
4 & $0.7 \%$ & $98.7 \%$ & $0.3 \%$ & $0.3 \%$ & $0.1 \%$ & $0.0 \%$ & 1.02743 & \\
5 & $0.7 \%$ & $98.7 \%$ & $0.2 \%$ & $0.1 \%$ & $0.3 \%$ & $0.0 \%$ & 1.02743 & \\
6 & $10.1 \%$ & $87.0 \%$ & $0.5 \%$ & $0.5 \%$ & $0.5 \%$ & $1.2 \%$ & 1.30293 & \\
7 & $0.0 \%$ & $0.0 \%$ & $0.0 \%$ & $0.0 \%$ & $0.0 \%$ & $100.0 \%$ & 0.99973 & \\
8 & $0.1 \%$ & $0.0 \%$ & $0.0 \%$ & $0.0 \%$ & $0.0 \%$ & $99.8 \%$ & 1.00308 & \\
9 & $0.7 \%$ & $0.0 \%$ & $0.3 \%$ & $0.3 \%$ & $0.1 \%$ & $98.7 \%$ & 1.02743 & \\
10 & $0.7 \%$ & $0.0 \%$ & $0.2 \%$ & $0.1 \%$ & $0.3 \%$ & $98.7 \%$ & 1.02743 & \\
11 & $10.1 \%$ & $1.2 \%$ & $0.5 \%$ & $0.5 \%$ & $0.5 \%$ & $87.0 \%$ & 1.30293 & \\
12 & $51.7 \%$ & $1.4 \%$ & $44.5 \%$ & $0.5 \%$ & $0.5 \%$ & $1.4 \%$ & 2.14734 & \\
13 & $51.7 \%$ & $1.4 \%$ & $0.5 \%$ & $44.5 \%$ & $0.5 \%$ & $1.4 \%$ & 2.14684 & \\
14 & $51.7 \%$ & $1.4 \%$ & $0.5 \%$ & $0.5 \%$ & $44.5 \%$ & $1.4 \%$ & 2.14857 & \\
\hline \hline
\end{tabular}


S1.6 $\mathbf{F e F}_{6}^{3-}$

S1.6.1 $\mathbf{F e F}_{6}^{3-}$ Oh High spin, $\left(\mathbf{t}_{2 g}^{3} \mathbf{e}_{g}^{2}-{ }^{6} A_{1 g}\right)$

\begin{tabular}{ccc}
\hline \hline \multicolumn{3}{l}{ 1-center FNO analysis, $\varepsilon=0.70000, \alpha$-orbitals } \\
\hline $\mathrm{MO}$ & $\lambda$ & Atoms \\
\hline 1 & 1.00000 & $\mathrm{Fe}_{1}$ \\
2 & 1.00000 & $\mathrm{Fe}_{1}$ \\
3 & 1.00000 & $\mathrm{Fe}_{1}$ \\
4 & 1.00000 & $\mathrm{Fe}_{1}$ \\
5 & 1.00000 & $\mathrm{Fe}_{1}$ \\
6 & 0.99885 & $\mathrm{Fe}_{1}$ \\
7 & 0.99709 & $\mathrm{Fe}_{1}$ \\
8 & 0.99709 & $\mathrm{Fe}_{1}$ \\
9 & 0.99709 & $\mathrm{Fe}_{1}$ \\
10 & 0.97411 & $\mathrm{Fe}_{1}$ \\
11 & 0.97411 & $\mathrm{Fe}_{1}$ \\
12 & 0.97411 & $\mathrm{Fe}_{1}$ \\
13 & 0.97035 & $\mathrm{Fe}_{1}$ \\
14 & 0.97035 & $\mathrm{Fe}_{1}$ \\
15 & 1.00120 & $\mathrm{~F}_{2}$ \\
16 & 1.00000 & $\mathrm{~F}_{2}$ \\
17 & 0.98602 & $\mathrm{~F}_{2}$ \\
18 & 0.98602 & $\mathrm{~F}_{2}$ \\
19 & 0.95217 & $\mathrm{~F}_{2}$ \\
20 & 1.00120 & $\mathrm{~F}_{3}$ \\
21 & 1.00000 & $\mathrm{~F}_{3}$ \\
22 & 0.98602 & $\mathrm{~F}_{3}$ \\
23 & 0.98602 & $\mathrm{~F}_{3}$ \\
24 & 0.95217 & $\mathrm{~F}_{3}$ \\
25 & 1.00120 & $\mathrm{~F}_{4}$ \\
26 & 1.00000 & $\mathrm{~F}_{4}$ \\
27 & 0.98602 & $\mathrm{~F}_{4}$ \\
28 & 0.98602 & $\mathrm{~F}_{4}$ \\
29 & 0.95217 & $\mathrm{~F}_{4}$ \\
30 & 1.00120 & $\mathrm{~F}_{5}$ \\
31 & 1.00000 & $\mathrm{~F}_{5}$ \\
32 & 0.98602 & $\mathrm{~F}_{5}$ \\
33 & 0.98602 & $\mathrm{~F}_{5}$ \\
34 & 0.95217 & $\mathrm{~F}_{5}$ \\
35 & 1.00120 & $\mathrm{~F}_{6}$ \\
36 & 1.00000 & $\mathrm{~F}_{6}$ \\
37 & 0.98602 & $\mathrm{~F}_{6}$ \\
38 & 0.98602 & $\mathrm{~F}_{6}$ \\
39 & 0.95217 & $\mathrm{~F}_{6}$ \\
40 & 1.00000 & $\mathrm{~F}_{7}$ \\
41 & 0.99805 & $\mathrm{~F}_{7}$ \\
42 & 0.98318 & $\mathrm{~F}_{7}$ \\
43 & 0.98318 & $\mathrm{~F}_{7}$ \\
44 & 0.95047 & $\mathrm{~F}_{7}$ \\
\hline \hline & &
\end{tabular}




\begin{tabular}{|c|c|c|c|c|c|c|c|c|}
\hline \multicolumn{9}{|c|}{ Localization and atoms expanded by each FNO, $\alpha$-orbitals } \\
\hline FNO & $\mathrm{Loc}_{1}$ & $\mathrm{LOC}_{2}$ & $\mathrm{Loc}_{3}$ & $\mathrm{Loc}_{4}$ & $\mathrm{LOc}_{5}$ & $\mathrm{Loc}_{6}$ & $\mathrm{Loc}_{7}$ & $n_{\text {eff }}$ \\
\hline 1 & $100.0 \%$ & $-0.0 \%$ & $-0.0 \%$ & $-0.0 \%$ & $-0.0 \%$ & $-0.0 \%$ & $-0.0 \%$ & 1.00000 \\
\hline 2 & $100.0 \%$ & $-0.0 \%$ & $-0.0 \%$ & $-0.0 \%$ & $-0.0 \%$ & $-0.0 \%$ & $0.0 \%$ & 1.00000 \\
\hline 3 & $100.0 \%$ & $-0.0 \%$ & $-0.0 \%$ & $-0.0 \%$ & $-0.0 \%$ & $-0.0 \%$ & $0.0 \%$ & 1.00000 \\
\hline 4 & $100.0 \%$ & $-0.0 \%$ & $-0.0 \%$ & $-0.0 \%$ & $-0.0 \%$ & $-0.0 \%$ & $0.0 \%$ & 1.00000 \\
\hline 5 & $100.0 \%$ & $-0.0 \%$ & $-0.0 \%$ & $-0.0 \%$ & $-0.0 \%$ & $-0.0 \%$ & $0.0 \%$ & 1.00000 \\
\hline 6 & $99.9 \%$ & $0.0 \%$ & $0.0 \%$ & $0.0 \%$ & $0.0 \%$ & $0.0 \%$ & $0.0 \%$ & 1.00231 \\
\hline 7 & $99.7 \%$ & $0.1 \%$ & $0.1 \%$ & $0.1 \%$ & $0.1 \%$ & $0.0 \%$ & $0.0 \%$ & 1.00584 \\
\hline 8 & $99.7 \%$ & $0.0 \%$ & $0.0 \%$ & $0.0 \%$ & $0.0 \%$ & $0.1 \%$ & $0.1 \%$ & 1.00584 \\
\hline 9 & $99.7 \%$ & $0.1 \%$ & $0.1 \%$ & $0.1 \%$ & $0.1 \%$ & $0.0 \%$ & $0.0 \%$ & 1.00584 \\
\hline 10 & $97.4 \%$ & $0.6 \%$ & $0.2 \%$ & $0.6 \%$ & $0.2 \%$ & $0.5 \%$ & $0.5 \%$ & 1.05371 \\
\hline 11 & $97.4 \%$ & $0.6 \%$ & $0.5 \%$ & $0.6 \%$ & $0.5 \%$ & $0.2 \%$ & $0.2 \%$ & 1.05371 \\
\hline 12 & $97.4 \%$ & $0.1 \%$ & $0.6 \%$ & $0.1 \%$ & $0.6 \%$ & $0.6 \%$ & $0.6 \%$ & 1.05370 \\
\hline 13 & $97.0 \%$ & $0.6 \%$ & $0.9 \%$ & $0.6 \%$ & $0.9 \%$ & $0.1 \%$ & $0.1 \%$ & 1.06182 \\
\hline 14 & $97.0 \%$ & $0.4 \%$ & $0.1 \%$ & $0.4 \%$ & $0.1 \%$ & $0.9 \%$ & $0.9 \%$ & 1.06182 \\
\hline 15 & $0.0 \%$ & $100.1 \%$ & $0.0 \%$ & $0.2 \%$ & $0.0 \%$ & $0.0 \%$ & $-0.3 \%$ & 0.99760 \\
\hline 16 & $-0.0 \%$ & $100.0 \%$ & $0.0 \%$ & $0.0 \%$ & $0.0 \%$ & $0.0 \%$ & $0.0 \%$ & 1.00000 \\
\hline 17 & $0.7 \%$ & $98.6 \%$ & $0.2 \%$ & $0.0 \%$ & $0.2 \%$ & $0.3 \%$ & $-0.0 \%$ & 1.02849 \\
\hline 18 & $0.7 \%$ & $98.6 \%$ & $0.3 \%$ & $0.0 \%$ & $0.3 \%$ & $0.2 \%$ & $-0.1 \%$ & 1.02849 \\
\hline 19 & $3.9 \%$ & $95.2 \%$ & $0.2 \%$ & $0.0 \%$ & $0.2 \%$ & $0.2 \%$ & $0.1 \%$ & 1.10107 \\
\hline 20 & $0.0 \%$ & $0.0 \%$ & $100.1 \%$ & $0.0 \%$ & $0.2 \%$ & $0.0 \%$ & $-0.3 \%$ & 0.99760 \\
\hline 21 & $-0.0 \%$ & $0.0 \%$ & $100.0 \%$ & $0.0 \%$ & $0.0 \%$ & $0.0 \%$ & $0.0 \%$ & 1.00000 \\
\hline 22 & $0.7 \%$ & $0.3 \%$ & $98.6 \%$ & $0.3 \%$ & $0.0 \%$ & $0.1 \%$ & $-0.2 \%$ & 1.02848 \\
\hline 23 & $0.7 \%$ & $0.1 \%$ & $98.6 \%$ & $0.1 \%$ & $0.0 \%$ & $0.3 \%$ & $0.1 \%$ & 1.02849 \\
\hline 24 & $3.9 \%$ & $0.2 \%$ & $95.2 \%$ & $0.2 \%$ & $0.0 \%$ & $0.2 \%$ & $0.1 \%$ & 1.10107 \\
\hline 25 & $0.0 \%$ & $0.2 \%$ & $0.0 \%$ & $100.1 \%$ & $0.0 \%$ & $0.0 \%$ & $-0.3 \%$ & 0.99760 \\
\hline 26 & $-0.0 \%$ & $0.0 \%$ & $0.0 \%$ & $100.0 \%$ & $0.0 \%$ & $0.0 \%$ & $0.0 \%$ & 1.00000 \\
\hline 27 & $0.7 \%$ & $0.0 \%$ & $0.4 \%$ & $98.6 \%$ & $0.4 \%$ & $0.1 \%$ & $-0.2 \%$ & 1.02847 \\
\hline 28 & $0.7 \%$ & $0.0 \%$ & $0.1 \%$ & $98.6 \%$ & $0.1 \%$ & $0.4 \%$ & $0.1 \%$ & 1.02849 \\
\hline 29 & $3.9 \%$ & $0.0 \%$ & $0.2 \%$ & $95.2 \%$ & $0.2 \%$ & $0.2 \%$ & $0.1 \%$ & 1.10107 \\
\hline 30 & $0.0 \%$ & $0.0 \%$ & $0.2 \%$ & $0.0 \%$ & $100.1 \%$ & $0.0 \%$ & $-0.3 \%$ & 0.99760 \\
\hline 31 & $-0.0 \%$ & $0.0 \%$ & $0.0 \%$ & $0.0 \%$ & $100.0 \%$ & $0.0 \%$ & $0.0 \%$ & 1.00000 \\
\hline 32 & $0.7 \%$ & $0.1 \%$ & $0.0 \%$ & $0.1 \%$ & $98.6 \%$ & $0.4 \%$ & $0.1 \%$ & 1.02849 \\
\hline 33 & $0.7 \%$ & $0.4 \%$ & $0.0 \%$ & $0.4 \%$ & $98.6 \%$ & $0.1 \%$ & $-0.2 \%$ & 1.02848 \\
\hline 34 & $3.9 \%$ & $0.2 \%$ & $0.0 \%$ & $0.2 \%$ & $95.2 \%$ & $0.2 \%$ & $0.1 \%$ & 1.10107 \\
\hline 35 & $0.0 \%$ & 0.0 & 0.0 & $0.0 \%$ & $0.0 \%$ & $100.1 \%$ & $-0.2 \%$ & 0.99761 \\
\hline 36 & $-0.0 \%$ & $0.0 \%$ & $0.0 \%$ & $0.0 \%$ & $0.0 \%$ & $100.0 \%$ & $0.0 \%$ & 1.00000 \\
\hline 37 & $0.7 \%$ & $0.4 \%$ & $0.1 \%$ & $0.4 \%$ & $0.1 \%$ & $98.6 \%$ & $-0.3 \%$ & 1.02847 \\
\hline 38 & $0.7 \%$ & $0.1 \%$ & $0.4 \%$ & $0.1 \%$ & $0.4 \%$ & $98.6 \%$ & $-0.3 \%$ & 1.02847 \\
\hline 39 & $3.9 \%$ & $0.2 \%$ & $0.2 \%$ & $0.2 \%$ & $0.2 \%$ & $95.2 \%$ & $-0.1 \%$ & 1.10106 \\
\hline 40 & $-0.0 \%$ & $0.0 \%$ & $0.0 \%$ & $0.0 \%$ & $0.0 \%$ & $0.0 \%$ & $100.0 \%$ & 1.00000 \\
\hline 41 & $0.0 \%$ & $0.0 \%$ & $0.0^{5}$ & $0.0 \%$ & $0.0 \%$ & $0.2 \%$ & $99.8 \%$ & 1.00391 \\
\hline 42 & $0.7^{9}$ & 0.1 & $0.4 \%$ & $0.1 \%$ & $0.4 \%$ & $0.0 \%$ & $98.3 \%$ & 1.03442 \\
\hline 43 & $0.7^{9}$ & $0.4 \%$ & $0.1 \%$ & $0.4 \%$ & $0.1 \%$ & $0.0 \%$ & $98.3 \%$ & 1.03442 \\
\hline 44 & $3.9 \%$ & $0.2 \%$ & $0.2 \%$ & $0.2 \%$ & $0.2 \%$ & $0.0 \%$ & $95.0 \%$ & 1.10500 \\
\hline
\end{tabular}




\begin{tabular}{cccc}
\hline \hline \multicolumn{3}{l}{ 1-center FNO } & analysis, $\varepsilon=0.70000, \beta-$ orbitals \\
\hline $\mathrm{MO}$ & $\lambda$ & Atoms \\
\hline 1 & 1.00000 & $\mathrm{Fe}_{1}$ \\
2 & 1.00000 & $\mathrm{Fe}_{1}$ \\
3 & 1.00000 & $\mathrm{Fe}_{1}$ \\
4 & 1.00000 & $\mathrm{Fe}_{1}$ \\
5 & 1.00000 & $\mathrm{Fe}_{1}$ \\
6 & 0.99879 & $\mathrm{Fe}_{1}$ \\
7 & 0.99658 & $\mathrm{Fe}_{1}$ \\
8 & 0.99658 & $\mathrm{Fe}_{1}$ \\
9 & 0.99658 & $\mathrm{Fe}_{1}$ \\
10 & 1.00091 & $\mathrm{~F}_{2}$ \\
11 & 1.00000 & $\mathrm{~F}_{2}$ \\
12 & 0.97579 & $\mathrm{~F}_{2}$ \\
13 & 0.97579 & $\mathrm{~F}_{2}$ \\
14 & 0.91584 & $\mathrm{~F}_{2}$ \\
15 & 1.00091 & $\mathrm{~F}_{3}$ \\
16 & 1.00000 & $\mathrm{~F}_{3}$ \\
17 & 0.97579 & $\mathrm{~F}_{3}$ \\
18 & 0.97579 & $\mathrm{~F}_{3}$ \\
19 & 0.91584 & $\mathrm{~F}_{3}$ \\
20 & 1.00091 & $\mathrm{~F}_{4}$ \\
21 & 1.00000 & $\mathrm{~F}_{4}$ \\
22 & 0.97579 & $\mathrm{~F}_{4}$ \\
23 & 0.97579 & $\mathrm{~F}_{4}$ \\
24 & 0.91584 & $\mathrm{~F}_{4}$ \\
25 & 1.00091 & $\mathrm{~F}_{5}$ \\
26 & 1.00000 & $\mathrm{~F}_{5}$ \\
27 & 0.97579 & $\mathrm{~F}_{5}$ \\
28 & 0.97579 & $\mathrm{~F}_{5}$ \\
29 & 0.91584 & $\mathrm{~F}_{5}$ \\
30 & 1.00091 & $\mathrm{~F}_{6}$ \\
31 & 1.00000 & $\mathrm{~F}_{6}$ \\
32 & 0.97579 & $\mathrm{~F}_{6}$ \\
33 & 0.97579 & $\mathrm{~F}_{6}$ \\
34 & 0.91584 & $\mathrm{~F}_{6}$ \\
35 & 1.00000 & $\mathrm{~F}_{7}$ \\
36 & 0.99730 & $\mathrm{~F}_{7}$ \\
37 & 0.97272 & $\mathrm{~F}_{7}$ \\
38 & 0.97272 & $\mathrm{~F}_{7}$ \\
39 & 0.91408 & $\mathrm{~F}_{7}$ \\
\hline \hline & &
\end{tabular}




\begin{tabular}{|c|c|c|c|c|c|c|c|c|}
\hline \multicolumn{9}{|c|}{ Localization and atoms expanded by each FNO, $\beta$-orbitals } \\
\hline FNO & $\mathrm{Loc}_{1}$ & $\mathrm{Loc}_{2}$ & $\mathrm{Loc}_{3}$ & $\mathrm{LOC}_{4}$ & $\mathrm{Loc}_{5}$ & $\mathrm{Loc}_{6}$ & $\mathrm{LOc}_{7}$ & $n_{\mathrm{eff}}$ \\
\hline 1 & $100.0 \%$ & $-0.0 \%$ & $-0.0 \%$ & $-0.0 \%$ & $-0.0 \%$ & $-0.0 \%$ & $-0.0 \%$ & 1.00000 \\
\hline 2 & $100.0 \%$ & $-0.0 \%$ & $-0.0 \%$ & $-0.0 \%$ & $-0.0 \%$ & $-0.0 \%$ & $0.0 \%$ & 1.00000 \\
\hline 3 & $100.0 \%$ & $-0.0 \%$ & $-0.0 \%$ & $-0.0 \%$ & $-0.0 \%$ & $-0.0 \%$ & $0.0 \%$ & 1.00000 \\
\hline 4 & $100.0 \%$ & $-0.0 \%$ & $-0.0 \%$ & $-0.0 \%$ & $-0.0 \%$ & $-0.0 \%$ & $0.0 \%$ & 1.00000 \\
\hline 5 & $100.0 \%$ & $0.0 \%$ & $0.0 \%$ & $0.0 \%$ & $0.0 \%$ & $0.0 \%$ & $0.0 \%$ & 1.00000 \\
\hline 6 & $99.9 \%$ & $0.0 \%$ & $0.0 \%$ & $0.0 \%$ & $0.0 \%$ & $0.0 \%$ & $0.0 \%$ & 1.00242 \\
\hline 7 & $99.7 \%$ & $0.0 \%$ & $0.1 \%$ & $0.0 \%$ & $0.1 \%$ & $0.0 \%$ & $0.0 \%$ & 1.00688 \\
\hline 8 & $99.7 \%$ & $0.1 \%$ & $0.0 \%$ & $0.1 \%$ & $0.0 \%$ & $0.1 \%$ & $0.1 \%$ & 1.00688 \\
\hline 9 & $99.7 \%$ & $0.1 \%$ & $0.0 \%$ & $0.1 \%$ & $0.0 \%$ & $0.1 \%$ & $0.1 \%$ & 1.00688 \\
\hline 10 & $0.1 \%$ & $100.1 \%$ & $0.0 \%$ & $0.2 \%$ & $0.0 \%$ & $0.0 \%$ & $-0.4 \%$ & 0.99817 \\
\hline 11 & $0.0 \%$ & 100. & 0.0 & $0.0 \%$ & $0.0 \%$ & $0.0 \%$ & $-0.0 \%$ & 1.00000 \\
\hline 12 & 1.5 & 97.6 & 0.1 & $0.0 \%$ & 0.1 & $0.5 \%$ & $0.2 \%$ & 1.04995 \\
\hline 13 & $1.5 \%$ & $97.6 \%$ & $0.5 \%$ & $0.0 \%$ & $0.5 \%$ & $0.1 \%$ & $-0.2 \%$ & 1.04993 \\
\hline 14 & $7.6 \%$ & $91.6 \%$ & $0.2 \%$ & $0.2 \%$ & $0.2 \%$ & $0.2 \%$ & $0.0 \%$ & 1.18414 \\
\hline 15 & $0.1 \%$ & $0.0 \%$ & $100.1 \%$ & $0.0 \%$ & $0.2 \%$ & $0.0 \%$ & $-0.4 \%$ & 0.99817 \\
\hline 16 & $0.0 \%$ & $0.0 \%$ & $100.0 \%$ & $0.0 \%$ & $0.0 \%$ & $0.0 \%$ & $-0.0 \%$ & 1.00000 \\
\hline 17 & 1.5 & & 97 & $0.5 \%$ & 0.0 & $0.1 \%$ & $-0.2 \%$ & 1.04993 \\
\hline 18 & 1.5 & 0 & 97.6 & $0.1 \%$ & 0.0 & 0.5 & $0.2 \%$ & 1.04995 \\
\hline 19 & $7.6 \%$ & $0.2 \%$ & $91.6 \%$ & $0.2 \%$ & $0.2 \%$ & $0.2 \%$ & $0.0 \%$ & 1.18414 \\
\hline 20 & $0.1 \%$ & $0.2 \%$ & $0.0 \%$ & $100.1 \%$ & $0.0 \%$ & $0.0 \%$ & $-0.4 \%$ & 0.99817 \\
\hline 21 & $0.0 \%$ & 0.0 & $0.0 \%$ & $100.0 \%$ & $0.0 \%$ & $0.0 \%$ & $-0.0 \%$ & 1.00000 \\
\hline 22 & $1.5 \%$ & 0.0 & $0.5 \%$ & $97.6 \%$ & $0.5 \%$ & $0.1 \%$ & $-0.2 \%$ & 1.04992 \\
\hline 23 & 1. & & & $97.6 \%$ & 0.1 & $0.5 \%$ & $0.2 \%$ & 1.04995 \\
\hline 24 & $7.6 \%$ & 0 & $0.2 \%$ & $91.6 \%$ & $0.2 \%$ & $0.2 \%$ & $0.0 \%$ & 1.18414 \\
\hline 25 & $0.1 \%$ & 0.0 & $0.2 \%$ & $0.0 \%$ & $100.1 \%$ & $0.0 \%$ & $-0.4 \%$ & 0.99817 \\
\hline 26 & $0.0 \%$ & 0.0 & 0.0 & $0.0 \%$ & $100.0 \%$ & $0.0 \%$ & $-0.0 \%$ & 1.00000 \\
\hline 27 & $1.5 \%$ & 0.4 & 0.0 & $0.4 \%$ & $97.6 \%$ & $0.2 \%$ & $-0.1 \%$ & 1.04994 \\
\hline 28 & $1.5 \%$ & $0.2 \%$ & $0.0 \%$ & $0.2 \%$ & $97.6 \%$ & $0.4 \%$ & $0.1 \%$ & 1.04996 \\
\hline 29 & & & 0.2 & $0.2 \%$ & $91.6 \%$ & $0.2 \%$ & $0.0 \%$ & 1.18414 \\
\hline 30 & 0.1 & & & $0.0 \%$ & $0.0 \%$ & $100.1 \%$ & $-0.2 \%$ & 0.99818 \\
\hline 31 & 0.0 & 0.0 & $0.0 \%$ & $0.0 \%$ & $0.0 \%$ & $100.0 \%$ & $0.0 \%$ & 1.00000 \\
\hline 32 & 1.5 & 0.5 & $0.1 \%$ & $0.5 \%$ & $0.1 \%$ & $97.6 \%$ & $-0.3 \%$ & 1.04992 \\
\hline 33 & $1.5 \%$ & 0.1 & $0.5 \%$ & $0.1 \%$ & $0.5 \%$ & $97.6 \%$ & $-0.3 \%$ & 1.04992 \\
\hline 34 & $7.6 \%$ & $0.2 \%$ & $0.2 \%$ & $0.2 \%$ & $0.2 \%$ & $91.6 \%$ & $0.0 \%$ & 1.18414 \\
\hline 35 & $-0.0 \%$ & 0.0 & 0.0 & $0.0 \%$ & $0.0 \%$ & $0.0 \%$ & $100.0 \%$ & 1.00000 \\
\hline 36 & 0.1 & 0.0 & 0.0 & $0.0 \%$ & $0.0 \%$ & $0.2 \%$ & $99.7 \%$ & 1.00541 \\
\hline 37 & & & 0.1 & $0.5 \%$ & $0.1 \%$ & $0.0 \%$ & $97.3 \%$ & 1.05657 \\
\hline 38 & & & 0.5 & $0.1 \%$ & $0.5 \%$ & $0.0 \%$ & $97.3 \%$ & 1.05657 \\
\hline 39 & $7.6 \%$ & $0.2 \%$ & $0.2 \%$ & $0.2 \%$ & $0.2 \%$ & $0.2 \%$ & $91.4 \%$ & 1.18868 \\
\hline
\end{tabular}


S1.6.2 $\quad \mathbf{F e F}_{6}^{3-} D_{4 h}$ Low spin $\left(\mathbf{e}_{g}^{4} \mathbf{b}_{2 g}^{1}-{ }^{2} B_{2 g}\right)$

\begin{tabular}{cccc}
\hline \hline 1-center FNO & analysis, $\varepsilon=0.70000 \alpha$-orbitals \\
\hline MO & $\lambda$ & Atoms $^{-}$ \\
\hline 1 & 1.00000 & $\mathrm{Fe}_{1}$ \\
2 & 1.00000 & $\mathrm{Fe}_{1}$ \\
3 & 1.00000 & $\mathrm{Fe}_{1}$ \\
4 & 1.00000 & $\mathrm{Fe}_{1}$ \\
5 & 1.00000 & $\mathrm{Fe}_{1}$ \\
6 & 0.99817 & $\mathrm{Fe}_{1}$ \\
7 & 0.99621 & $\mathrm{Fe}_{1}$ \\
8 & 0.99544 & $\mathrm{Fe}_{1}$ \\
9 & 0.99544 & $\mathrm{Fe}_{1}$ \\
10 & 0.96609 & $\mathrm{Fe}_{1}$ \\
11 & 0.96609 & $\mathrm{Fe}_{1}$ \\
12 & 0.96536 & $\mathrm{Fe}_{1}$ \\
13 & 1.00067 & $\mathrm{~F}_{2}$ \\
14 & 1.00000 & $\mathrm{~F}_{2}$ \\
15 & 0.98433 & $\mathrm{~F}_{2}$ \\
16 & 0.98409 & $\mathrm{~F}_{2}$ \\
17 & 0.89917 & $\mathrm{~F}_{2}$ \\
18 & 1.00069 & $\mathrm{~F}_{3}$ \\
19 & 1.00000 & $\mathrm{~F}_{3}$ \\
20 & 0.98465 & $\mathrm{~F}_{3}$ \\
21 & 0.98465 & $\mathrm{~F}_{3}$ \\
22 & 0.90925 & $\mathrm{~F}_{3}$ \\
23 & 1.00067 & $\mathrm{~F}_{4}$ \\
24 & 1.00000 & $\mathrm{~F}_{4}$ \\
25 & 0.98433 & $\mathrm{~F}_{4}$ \\
26 & 0.98409 & $\mathrm{~F}_{4}$ \\
27 & 0.89917 & $\mathrm{~F}_{4}$ \\
28 & 1.00069 & $\mathrm{~F}_{5}$ \\
29 & 1.00000 & $\mathrm{~F}_{5}$ \\
30 & 0.98465 & $\mathrm{~F}_{5}$ \\
31 & 0.98465 & $\mathrm{~F}_{5}$ \\
32 & 0.90925 & $\mathrm{~F}_{5}$ \\
33 & 1.00067 & $\mathrm{~F}_{6}$ \\
34 & 1.00000 & $\mathrm{~F}_{6}$ \\
35 & 0.98433 & $\mathrm{~F}_{6}$ \\
36 & 0.98409 & $\mathrm{~F}_{6}$ \\
37 & 0.89917 & $\mathrm{~F}_{6}$ \\
38 & 1.00000 & $\mathrm{~F}_{7}$ \\
39 & 0.99634 & $\mathrm{~F}_{7}$ \\
40 & 0.98375 & $\mathrm{~F}_{7}$ \\
41 & 0.98167 & $\mathrm{~F}_{7}$ \\
42 & 0.89822 & $\mathrm{~F}_{7}$ \\
\hline \hline & &
\end{tabular}




\begin{tabular}{|c|c|c|c|c|c|c|c|c|}
\hline \multicolumn{9}{|c|}{ Localization and atoms expanded by each FNO $\alpha$-orbitals } \\
\hline FNO & $\mathrm{Loc}_{1}$ & $\mathrm{Loc}_{2}$ & $\mathrm{Loc}_{3}$ & $\mathrm{LOC}_{4}$ & $\mathrm{Loc}_{5}$ & $\mathrm{Loc}_{6}$ & $\mathrm{Loc}_{7}$ & $n_{\mathrm{eff}}$ \\
\hline 1 & $100.0 \%$ & $-0.0 \%$ & $-0.0 \%$ & $-0.0 \%$ & $-0.0 \%$ & $-0.0 \%$ & $0.0 \%$ & 1.00000 \\
\hline 2 & $100.0 \%$ & $-0.0 \%$ & $-0.0 \%$ & $-0.0 \%$ & $-0.0 \%$ & $-0.0 \%$ & $0.0 \%$ & 1.00000 \\
\hline 3 & $100.0 \%$ & $-0.0 \%$ & $-0.0 \%$ & $-0.0 \%$ & $-0.0 \%$ & $-0.0 \%$ & $0.0 \%$ & 1.00000 \\
\hline 4 & $100.0 \%$ & $-0.0 \%$ & $-0.0 \%$ & $-0.0 \%$ & $-0.0 \%$ & $-0.0 \%$ & $0.0 \%$ & 1.00000 \\
\hline 5 & $100.0 \%$ & $-0.0 \%$ & $0.0 \%$ & $-0.0 \%$ & $0.0 \%$ & $-0.0 \%$ & $0.0 \%$ & 1.00000 \\
\hline 6 & $99.8 \%$ & $0.0 \%$ & $0.0 \%$ & $0.0 \%$ & $0.0 \%$ & $0.0 \%$ & $0.0 \%$ & 1.00367 \\
\hline 7 & $99.6 \%$ & $0.0 \%$ & $0.1 \%$ & $0.0 \%$ & $0.1 \%$ & $0.0 \%$ & $0.0 \%$ & 1.00762 \\
\hline 8 & $99.5 \%$ & $0.1 \%$ & $0.0 \%$ & $0.1 \%$ & $0.0 \%$ & $0.1 \%$ & $0.1 \%$ & 1.00917 \\
\hline 9 & $99.5 \%$ & $0.1 \%$ & $0.0 \%$ & $0.1 \%$ & $0.0 \%$ & $0.1 \%$ & $0.1 \%$ & 1.00917 \\
\hline 10 & $96.6 \%$ & $0.9 \%$ & $0.7 \%$ & $0.9 \%$ & $0.7 \%$ & $0.1 \%$ & $0.1 \%$ & 1.07115 \\
\hline 11 & $96.6 \%$ & $0.1 \%$ & $0.7 \%$ & $0.1 \%$ & $0.7 \%$ & $0.9 \%$ & $0.9 \%$ & 1.07115 \\
\hline 12 & $96.5 \%$ & $0.8 \%$ & $0.1 \%$ & $0.8 \%$ & $0.1 \%$ & $0.8 \%$ & $0.8 \%$ & 1.07273 \\
\hline 13 & $0.1 \%$ & $100.1 \%$ & $0.0 \%$ & $0.2 \%$ & $0.0 \%$ & $0.0 \%$ & $-0.4 \%$ & 0.99864 \\
\hline 14 & $0.0 \%$ & $100.0 \%$ & $0.0 \%$ & $0.0 \%$ & $0.0 \%$ & $0.0 \%$ & $-0.0 \%$ & 1.00000 \\
\hline 15 & $0.8 \%$ & $98.4 \%$ & $0.1 \%$ & $0.0 \%$ & $0.1 \%$ & $0.5 \%$ & $0.2 \%$ & 1.03199 \\
\hline 16 & $0.6 \%$ & $98.4 \%$ & $0.4 \%$ & $0.0 \%$ & $0.4 \%$ & $0.1 \%$ & $0.0 \%$ & 1.03252 \\
\hline 17 & $9.0 \%$ & 89.9 & $0.2 \%$ & $0.4 \%$ & $0.2 \%$ & $0.2 \%$ & $0.1 \%$ & 1.22460 \\
\hline 18 & $0.1 \%$ & $0.0 \%$ & $100.1 \%$ & $0.0 \%$ & $0.2 \%$ & $0.0 \%$ & $-0.4 \%$ & 0.99861 \\
\hline 19 & $0.0 \%$ & $0.0 \%$ & $100.0 \%$ & $0.0 \%$ & $0.0 \%$ & $0.0 \%$ & $-0.0 \%$ & 1.00000 \\
\hline 20 & $0.5 \%$ & $0.4 \%$ & $98.5 \%$ & $0.4 \%$ & $0.0 \%$ & $0.1 \%$ & $0.1 \%$ & 1.03136 \\
\hline 21 & $0.5 \%$ & $0.1 \%$ & $98.5 \%$ & $0.1 \%$ & $0.0 \%$ & $0.4 \%$ & $0.4 \%$ & 1.03136 \\
\hline 22 & $8.0 \%$ & & $90.9 \%$ & $0.2 \%$ & $0.3 \%$ & $0.2 \%$ & $0.2 \%$ & 1.20021 \\
\hline 23 & $0.1 \%$ & 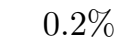 & $0.0 \%$ & $100.1 \%$ & $0.0 \%$ & $0.0 \%$ & $-0.4 \%$ & 0.99864 \\
\hline 24 & $0.0 \%$ & 0.0 & $0.0 \%$ & $100.0 \%$ & $0.0 \%$ & $0.0 \%$ & $-0.0 \%$ & 1.00000 \\
\hline 25 & $0.8 \%$ & 0.0 & $0.1 \%$ & $98.4 \%$ & $0.1 \%$ & $0.5 \%$ & $0.2 \%$ & 1.03199 \\
\hline 26 & $0.6 \%$ & 0.0 & $0.4 \%$ & $98.4 \%$ & $0.4 \%$ & $0.1 \%$ & $0.0 \%$ & 1.03252 \\
\hline 27 & $9.0 \%$ & 0.4 & $0.2 \%$ & $89.9 \%$ & $0.2 \%$ & $0.2 \%$ & $0.1 \%$ & 1.22460 \\
\hline 28 & $0.1 \%$ & & $0.2 \%$ & $0.0 \%$ & $100.1 \%$ & $0.0 \%$ & $-0.4 \%$ & 0.99861 \\
\hline 29 & 0 . & 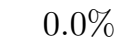 & $0.0 \%$ & $0.0 \%$ & $100.0 \%$ & $0.0 \%$ & $-0.0 \%$ & 1.0 \\
\hline 30 & $0.5 \%$ & 0.1 & $0.0 \%$ & $0.1 \%$ & $98.5 \%$ & $0.4 \%$ & $0.4 \%$ & 1.03135 \\
\hline 31 & $0.5 \%$ & 0.4 & $0.0 \%$ & $0.4 \%$ & $98.5 \%$ & $0.1 \%$ & $0.1 \%$ & 1.03135 \\
\hline 32 & $8.0 \%$ & 02 & $0.3 \%$ & $0.2 \%$ & $90.9 \%$ & $0.2 \%$ & $0.2 \%$ & 1.20021 \\
\hline 33 & $0.1 \%$ & 0. & $0.0 \%$ & $0.0 \%$ & $0.0 \%$ & $100.1 \%$ & $-0.2 \%$ & 0.99866 \\
\hline 34 & $0.0 \%$ & & $0.0 \%$ & $0.0 \%$ & $0.0 \%$ & $100.0 \%$ & $0.0 \%$ & 1.00000 \\
\hline 35 & 0.8 & & & $0.5 \%$ & $0.1 \%$ & $98.4 \%$ & $-0.3 \%$ & 1.03197 \\
\hline 36 & $0.6^{c}$ & & $0.4 \%$ & $0.1 \%$ & $0.4 \%$ & $98.4 \%$ & $-0.0 \%$ & 1.03252 \\
\hline 37 & $9.0 \%$ & 0. & $0.2 \%$ & $0.2 \%$ & $0.2 \%$ & $89.9 \%$ & $0.3 \%$ & 1.22461 \\
\hline 38 & $-0.0 \%$ & 0 & $-0.0 \%$ & $0.0 \%$ & $-0.0 \%$ & $0.0 \%$ & $100.0 \%$ & 1.00000 \\
\hline 39 & $0.1 \%$ & $0.0 \%$ & $0.0 \%$ & $0.0 \%$ & $0.0 \%$ & $0.2 \%$ & $99.6 \%$ & 1.00736 \\
\hline 40 & $0.6 \%$ & 0.1 & $0.4 \%$ & $0.1 \%$ & $0.4 \%$ & $0.0 \%$ & $98.4 \%$ & 1.03322 \\
\hline 41 & $0.8 \%$ & 0.5 & $0.1 \%$ & $0.5 \%$ & $0.1 \%$ & $0.0 \%$ & $98.2 \%$ & 1.03757 \\
\hline 42 & $9.0 \%$ & $0.2 \%$ & $0.2 \%$ & $0.2 \%$ & $0.2 \%$ & $0.4 \%$ & $89.8 \%$ & 1.22715 \\
\hline
\end{tabular}




\begin{tabular}{cccl}
\hline \hline \multicolumn{5}{c}{ 1-center FNO analysis, $\varepsilon=0.70000$} & $\beta$-orbitals \\
\hline $\mathrm{MO}$ & $\lambda$ & Atoms \\
\hline 1 & 1.00000 & $\mathrm{Fe}_{1}$ \\
2 & 1.00000 & $\mathrm{Fe}_{1}$ \\
3 & 1.00000 & $\mathrm{Fe}_{1}$ \\
4 & 1.00000 & $\mathrm{Fe}_{1}$ \\
5 & 1.00000 & $\mathrm{Fe}_{1}$ \\
6 & 0.99814 & $\mathrm{Fe}_{1}$ \\
7 & 0.99612 & $\mathrm{Fe}_{1}$ \\
8 & 0.99522 & $\mathrm{Fe}_{1}$ \\
9 & 0.99522 & $\mathrm{Fe}_{1}$ \\
10 & 0.96362 & $\mathrm{Fe}_{1}$ \\
11 & 0.96362 & $\mathrm{Fe}_{1}$ \\
12 & 1.00096 & $\mathrm{~F}_{2}$ \\
13 & 1.00000 & $\mathrm{~F}_{2}$ \\
14 & 0.98412 & $\mathrm{~F}_{2}$ \\
15 & 0.97233 & $\mathrm{~F}_{2}$ \\
16 & 0.91076 & $\mathrm{~F}_{2}$ \\
17 & 1.00084 & $\mathrm{~F}_{3}$ \\
18 & 1.00000 & $\mathrm{~F}_{3}$ \\
19 & 0.98479 & $\mathrm{~F}_{3}$ \\
20 & 0.98479 & $\mathrm{~F}_{3}$ \\
21 & 0.91798 & $\mathrm{~F}_{3}$ \\
22 & 1.00096 & $\mathrm{~F}_{4}$ \\
23 & 1.00000 & $\mathrm{~F}_{4}$ \\
24 & 0.98412 & $\mathrm{~F}_{4}$ \\
25 & 0.97233 & $\mathrm{~F}_{4}$ \\
26 & 0.91076 & $\mathrm{~F}_{4}$ \\
27 & 1.00084 & $\mathrm{~F}_{5}$ \\
28 & 1.00000 & $\mathrm{~F}_{5}$ \\
29 & 0.98479 & $\mathrm{~F}_{5}$ \\
30 & 0.98479 & $\mathrm{~F}_{5}$ \\
31 & 0.91798 & $\mathrm{~F}_{5}$ \\
32 & 1.00096 & $\mathrm{~F}_{6}$ \\
33 & 1.00000 & $\mathrm{~F}_{6}$ \\
34 & 0.98412 & $\mathrm{~F}_{6}$ \\
35 & 0.97233 & $\mathrm{~F}_{6}$ \\
36 & 0.91076 & $\mathrm{~F}_{6}$ \\
37 & 1.00000 & $\mathrm{~F}_{7}$ \\
38 & 0.99659 & $\mathrm{~F}_{7}$ \\
39 & 0.98379 & $\mathrm{~F}_{7}$ \\
40 & 0.96955 & $\mathrm{~F}_{7}$ \\
41 & 0.90974 & $\mathrm{~F}_{7}$ \\
\hline \hline
\end{tabular}




\begin{tabular}{|c|c|c|c|c|c|c|c|c|}
\hline \multicolumn{9}{|c|}{ Localization and atoms expanded by each FNO, $\beta$-orbitals } \\
\hline FNO & $\mathrm{Loc}_{1}$ & $\mathrm{Loc}_{2}$ & $\mathrm{Loc}_{3}$ & $\mathrm{LOC}_{4}$ & $\mathrm{Loc}_{5}$ & $\mathrm{Loc}_{6}$ & $\mathrm{Loc}_{7}$ & $n_{\mathrm{eff}}$ \\
\hline 1 & $100.0 \%$ & $-0.0 \%$ & $-0.0 \%$ & $-0.0 \%$ & $-0.0 \%$ & $-0.0 \%$ & $-0.0 \%$ & 1.00000 \\
\hline 2 & $100.0 \%$ & $-0.0 \%$ & $0.0 \%$ & $-0.0 \%$ & $0.0 \%$ & $-0.0 \%$ & $0.0 \%$ & 1.00000 \\
\hline 3 & $100.0 \%$ & $-0.0 \%$ & $0.0 \%$ & $-0.0 \%$ & $0.0 \%$ & $-0.0 \%$ & $0.0 \%$ & 1.00000 \\
\hline 4 & $100.0 \%$ & $-0.0 \%$ & $-0.0 \%$ & $-0.0 \%$ & $-0.0 \%$ & $-0.0 \%$ & $0.0 \%$ & 1.00000 \\
\hline 5 & $100.0 \%$ & $-0.0 \%$ & $-0.0 \%$ & $-0.0 \%$ & $-0.0 \%$ & $-0.0 \%$ & $0.0 \%$ & 1.00000 \\
\hline 6 & $99.8 \%$ & $0.0 \%$ & $0.0 \%$ & $0.0 \%$ & $0.0 \%$ & $0.0 \%$ & $0.0 \%$ & 1.00372 \\
\hline 7 & $99.6 \%$ & $0.0 \%$ & $0.2 \%$ & $0.0 \%$ & $0.2 \%$ & $0.0 \%$ & $0.0 \%$ & 1.00781 \\
\hline 8 & $99.5 \%$ & $0.1 \%$ & $0.0 \%$ & $0.1 \%$ & $0.0 \%$ & $0.2 \%$ & $0.2 \%$ & 1.00962 \\
\hline 9 & $99.5 \%$ & $0.2 \%$ & $0.0 \%$ & $0.2 \%$ & $0.0 \%$ & $0.1 \%$ & $0.1 \%$ & 1.00962 \\
\hline 10 & $96.4 \%$ & $0.5 \%$ & $0.8 \%$ & $0.5 \%$ & $0.8 \%$ & $0.6 \%$ & $0.6 \%$ & 1.07666 \\
\hline 11 & $96.4 \%$ & $0.6 \%$ & $0.8 \%$ & $0.6 \%$ & $0.8 \%$ & $0.5 \%$ & $0.5 \%$ & 1.07666 \\
\hline 12 & $0.1 \%$ & $100.1 \%$ & $0.0 \%$ & $0.2 \%$ & $0.0 \%$ & $0.0 \%$ & $-0.4 \%$ & 0.99806 \\
\hline 13 & $0.0 \%$ & $100.0 \%$ & $0.0 \%$ & $0.0 \%$ & $0.0 \%$ & $0.0 \%$ & $0.0 \%$ & 1.00000 \\
\hline 14 & $0.6 \%$ & $98.4 \%$ & $0.4 \%$ & $0.0 \%$ & $0.4 \%$ & $0.1 \%$ & $0.0 \%$ & 1.03245 \\
\hline 15 & $1.8 \%$ & $97.2 \%$ & $0.1 \%$ & $0.0 \%$ & $0.1 \%$ & $0.6 \%$ & $0.3 \%$ & 1.05735 \\
\hline 16 & $8.0 \%$ & $91.1 \%$ & $0.2 \%$ & $0.3 \%$ & $0.2 \%$ & $0.2 \%$ & $0.1 \%$ & 1.19627 \\
\hline 17 & $0.1 \%$ & $0.0 \%$ & $100.1 \%$ & $0.0 \%$ & $0.2 \%$ & $0.0 \%$ & $-0.4 \%$ & 0.99830 \\
\hline 18 & $0.0 \%$ & $0.0 \%$ & $100.0 \%$ & $0.0 \%$ & $0.0 \%$ & $0.0 \%$ & $-0.0 \%$ & 1.00000 \\
\hline 19 & $0.5 \%$ & $0.4 \%$ & $98.5 \%$ & $0.4 \%$ & $0.0 \%$ & $0.1 \%$ & $0.1 \%$ & 1.03106 \\
\hline 20 & $0.5 \%$ & $0.1 \%$ & $98.5 \%$ & $0.1 \%$ & $0.0 \%$ & $0.4 \%$ & $0.4 \%$ & 1.03106 \\
\hline 21 & $7.3 \%$ & $0.2 \%$ & $91.8 \%$ & $0.2 \%$ & $0.2 \%$ & $0.2 \%$ & $0.2 \%$ & 1.17925 \\
\hline 22 & $0.1 \%$ & $0.2 \%$ & $0.0 \%$ & $100.1 \%$ & $0.0 \%$ & $0.0 \%$ & $-0.4 \%$ & 0.99806 \\
\hline 23 & $0.0 \%$ & $0.0 \%$ & $0.0 \%$ & $100.0 \%$ & $0.0 \%$ & $0.0 \%$ & $0.0 \%$ & 1.00000 \\
\hline 24 & $0.6 \%$ & $0.0 \%$ & $0.4 \%$ & $98.4 \%$ & $0.4 \%$ & $0.1 \%$ & $0.0 \%$ & 1.03245 \\
\hline 25 & $1.8 \%$ & $0.0 \%$ & $0.1 \%$ & $97.2 \%$ & $0.1 \%$ & $0.6 \%$ & $0.3 \%$ & 1.05735 \\
\hline 26 & $8.0 \%$ & $0.3 \%$ & $0.2 \%$ & $91.1 \%$ & $0.2 \%$ & $0.2 \%$ & $0.1 \%$ & 1.19627 \\
\hline 27 & $0.1 \%$ & $0.0 \%$ & $0.2 \%$ & $0.0 \%$ & $100.1 \%$ & $0.0 \%$ & $-0.4 \%$ & 0.99830 \\
\hline 28 & $0.0 \%$ & $0.0 \%$ & $0.0 \%$ & $0.0 \%$ & $100.0 \%$ & $0.0 \%$ & $-0.0 \%$ & 1.00000 \\
\hline 29 & $0.5 \%$ & $0.1 \%$ & $0.0 \%$ & $0.1 \%$ & $98.5 \%$ & $0.4 \%$ & $0.4 \%$ & 1.03105 \\
\hline 30 & $0.5 \%$ & $0.4 \%$ & $0.0 \%$ & $0.4 \%$ & $98.5 \%$ & $0.1 \%$ & $0.1 \%$ & 1.03105 \\
\hline 31 & $7.3 \%$ & $0.2 \%$ & $0.2 \%$ & $0.2 \%$ & $91.8 \%$ & $0.2 \%$ & $0.2 \%$ & 1.17925 \\
\hline 32 & $0.1 \%$ & $0.0 \%$ & $0.0 \%$ & $0.0 \%$ & $0.0 \%$ & $100.1 \%$ & $-0.2 \%$ & 0.99808 \\
\hline 33 & $0.0 \%$ & $0.0 \%$ & $0.0 \%$ & $0.0 \%$ & $0.0 \%$ & $100.0 \%$ & $0.0 \%$ & 1.00000 \\
\hline 34 & $0.6 \%$ & $0.1 \%$ & $0.4 \%$ & $0.1 \%$ & $0.4 \%$ & $98.4 \%$ & $-0.0 \%$ & 1.03244 \\
\hline 35 & $1.8 \%$ & 0.6 & $0.1 \%$ & $0.6 \%$ & $0.1 \%$ & $97.2 \%$ & $-0.3 \%$ & 1.05731 \\
\hline 36 & $8.0 \%$ & $0.2 \%$ & $0.2 \%$ & $0.2 \%$ & $0.2 \%$ & $91.1 \%$ & $0.2 \%$ & 1.19627 \\
\hline 37 & $-0.0 \%$ & $0.0 \%$ & $-0.0 \%$ & $0.0 \%$ & $-0.0 \%$ & $0.0 \%$ & $100.0 \%$ & 1.00000 \\
\hline 38 & $0.1 \%$ & $0.0 \%$ & $0.0 \%$ & $0.0 \%$ & $0.0 \%$ & $0.2 \%$ & $99.7 \%$ & 1.00685 \\
\hline 39 & $0.6 \%$ & $0.1 \%$ & $0.4 \%$ & $0.1 \%$ & $0.4 \%$ & $0.0 \%$ & $98.4 \%$ & 1.03315 \\
\hline 40 & $1.7 \%$ & $0.6 \%$ & $0.1 \%$ & $0.6 \%$ & $0.1 \%$ & $0.0 \%$ & $97.0 \%$ & 1.06337 \\
\hline 41 & $8.0 \%$ & $0.2 \%$ & $0.2 \%$ & $0.2 \%$ & $0.2 \%$ & $0.3 \%$ & $91.0 \%$ & 1.19893 \\
\hline
\end{tabular}


$\mathrm{S} 1.7 \mathrm{PtO}_{4}^{2+}$

\begin{tabular}{|c|c|c|}
\hline \multicolumn{3}{|c|}{1 1-center FNO analysis, $\varepsilon=0.90000$} \\
\hline $\mathrm{MO}$ & $\lambda$ & Atoms \\
\hline 1 & 1.00000 & $\mathrm{Pt}_{1}$ \\
\hline 2 & 1.00000 & $\mathrm{Pt}_{1}$ \\
\hline 3 & 1.00000 & $\mathrm{Pt}_{1}$ \\
\hline 4 & 1.00000 & $\mathrm{Pt}_{1}$ \\
\hline 5 & 1.00000 & $\mathrm{Pt}_{1}$ \\
\hline 6 & 1.00000 & $\mathrm{Pt}_{1}$ \\
\hline 7 & 1.00000 & $\mathrm{Pt}_{1}$ \\
\hline 8 & 1.00000 & $\mathrm{Pt}_{1}$ \\
\hline 9 & 1.00000 & $\mathrm{Pt}_{1}$ \\
\hline 10 & 1.00000 & $\mathrm{Pt}_{1}$ \\
\hline 11 & 1.00000 & $\mathrm{Pt}_{1}$ \\
\hline 12 & 1.00000 & $\mathrm{Pt}_{1}$ \\
\hline 13 & 1.00000 & $\mathrm{Pt}_{1}$ \\
\hline 14 & 1.00000 & $\mathrm{Pt}_{1}$ \\
\hline 15 & 1.00000 & $\mathrm{Pt}_{1}$ \\
\hline 16 & 0.99999 & $\mathrm{Pt}_{1}$ \\
\hline 17 & 0.99999 & $\mathrm{Pt}_{1}$ \\
\hline 18 & 0.99999 & $\mathrm{Pt}_{1}$ \\
\hline 19 & 0.99998 & $\mathrm{Pt}_{1}$ \\
\hline 20 & 0.99997 & $\mathrm{Pt}_{1}$ \\
\hline 21 & 0.99997 & $\mathrm{Pt}_{1}$ \\
\hline 22 & 0.99997 & $\mathrm{Pt}_{1}$ \\
\hline 23 & 0.99996 & $\mathrm{Pt}_{1}$ \\
\hline 24 & 0.99996 & $\mathrm{Pt}_{1}$ \\
\hline 25 & 0.99996 & $\mathrm{Pt}_{1}$ \\
\hline 26 & 0.99995 & $\mathrm{Pt}_{1}$ \\
\hline 27 & 0.99995 & $\mathrm{Pt}_{1}$ \\
\hline 28 & 0.99995 & $\mathrm{Pt}_{1}$ \\
\hline 29 & 0.99994 & $\mathrm{Pt}_{1}$ \\
\hline 30 & 0.99994 & $\mathrm{Pt}_{1}$ \\
\hline 31 & 0.99701 & $\mathrm{Pt}_{1}$ \\
\hline 32 & 0.99388 & $\mathrm{Pt}_{1}$ \\
\hline 33 & 0.99388 & $\mathrm{Pt}_{1}$ \\
\hline 34 & 0.99387 & $\mathrm{Pt}_{1}$ \\
\hline 35 & 1.00000 & $\mathrm{O}_{2}$ \\
\hline 36 & 0.98486 & $\mathrm{O}_{2}$ \\
\hline 37 & 1.00000 & $\mathrm{O}_{3}$ \\
\hline 38 & 0.98487 & $\mathrm{O}_{3}$ \\
\hline 39 & 1.00000 & $\mathrm{O}_{4}$ \\
\hline 40 & 0.98488 & $\mathrm{O}_{4}$ \\
\hline 41 & 1.00000 & $\mathrm{O}_{5}$ \\
\hline 42 & 0.98487 & $\mathrm{O}_{5}$ \\
\hline \multicolumn{3}{|c|}{ 1-center FNO analysis, $\varepsilon=0.90000$} \\
\hline $\mathrm{MO}$ & $\lambda$ & Atoms \\
\hline 43 & 0.98974 & $\mathrm{Pt}_{1}-\mathrm{O}_{2}$ \\
\hline 44 & 0.98207 & $\mathrm{Pt}_{1}-\mathrm{O}_{2}$ \\
\hline 45 & 0.98206 & $\mathrm{Pt}_{1}-\mathrm{O}_{2}$ \\
\hline 46 & 0.98975 & $\mathrm{Pt}_{1}-\mathrm{O}_{3}$ \\
\hline 47 & 0.98206 & $\mathrm{Pt}_{1}-\mathrm{O}_{3}$ \\
\hline 48 & 0.98206 & $\mathrm{Pt}_{1}-\mathrm{O}_{3}$ \\
\hline 49 & 0.98975 & $\mathrm{Pt}_{1}-\mathrm{O}_{4}$ \\
\hline 50 & 0.98206 & $\mathrm{Pt}_{1}-\mathrm{O}_{4}$ \\
\hline 51 & 0.98206 & $\mathrm{Pt}_{1}-\mathrm{O}_{4}$ \\
\hline 52 & 0.98975 & $\mathrm{Pt}_{1}-\mathrm{O}_{5}$ \\
\hline 53 & 0.98207 & $\mathrm{Pt}_{1}-\mathrm{O}_{5}$ \\
\hline 54 & 0.98206 & $\mathrm{Pt}_{1}-\mathrm{O}_{5}$ \\
\hline
\end{tabular}




\begin{tabular}{|c|c|c|c|c|c|c|}
\hline \multicolumn{7}{|c|}{ Localization and atoms expanded by each FNO } \\
\hline $\mathrm{FNO}$ & $\mathrm{LOc}_{1}$ & $\mathrm{LOC}_{2}$ & $\mathrm{Loc}_{3}$ & $\mathrm{LOC}_{4}$ & $\mathrm{LOC}_{5}$ & $n_{\mathrm{eff}}$ \\
\hline 1 & $100.0 \%$ & $0.0 \%$ & $0.0 \%$ & $0.0 \%$ & $0.0 \%$ & 1.00000 \\
\hline 2 & $100.0 \%$ & $0.0 \%$ & $0.0 \%$ & $0.0 \%$ & $0.0 \%$ & 1.00000 \\
\hline 3 & $100.0 \%$ & $0.0 \%$ & $0.0 \%$ & $0.0 \%$ & $0.0 \%$ & 1.00000 \\
\hline 4 & $100.0 \%$ & $0.0 \%$ & $0.0 \%$ & $0.0 \%$ & $0.0 \%$ & 1.00000 \\
\hline 5 & $100.0 \%$ & $0.0 \%$ & $0.0 \%$ & $0.0 \%$ & $0.0 \%$ & 1.00000 \\
\hline 6 & $100.0 \%$ & $0.0 \%$ & $0.0 \%$ & $0.0 \%$ & $0.0 \%$ & 1.00000 \\
\hline 7 & $100.0 \%$ & $0.0 \%$ & $0.0 \%$ & $0.0 \%$ & $0.0 \%$ & 1.00000 \\
\hline 8 & $100.0 \%$ & $0.0 \%$ & $0.0 \%$ & $0.0 \%$ & $0.0 \%$ & 1.00000 \\
\hline 9 & $100.0 \%$ & $0.0 \%$ & $0.0 \%$ & $0.0 \%$ & $0.0 \%$ & 1.00000 \\
\hline 10 & $100.0 \%$ & $0.0 \%$ & $0.0 \%$ & $0.0 \%$ & $0.0 \%$ & 1.00000 \\
\hline 11 & $100.0 \%$ & $0.0 \%$ & $0.0 \%$ & $0.0 \%$ & $0.0 \%$ & 1.00000 \\
\hline 12 & $100.0 \%$ & $0.0 \%$ & $0.0 \%$ & $0.0 \%$ & $0.0 \%$ & 1.00000 \\
\hline 13 & $100.0 \%$ & $0.0 \%$ & $0.0 \%$ & $0.0 \%$ & $0.0 \%$ & 1.00000 \\
\hline 14 & $100.0 \%$ & $0.0 \%$ & $0.0 \%$ & $0.0 \%$ & $0.0 \%$ & 1.00000 \\
\hline 15 & $100.0 \%$ & $0.0 \%$ & $0.0 \%$ & $0.0 \%$ & $0.0 \%$ & 1.00001 \\
\hline 16 & $100.0 \%$ & $0.0 \%$ & $0.0 \%$ & $0.0 \%$ & $0.0 \%$ & 1.00003 \\
\hline 17 & $100.0 \%$ & $0.0 \%$ & $0.0 \%$ & $0.0 \%$ & $0.0 \%$ & 1.00003 \\
\hline 18 & $100.0 \%$ & $0.0 \%$ & $0.0 \%$ & $0.0 \%$ & $0.0 \%$ & 1.00003 \\
\hline 19 & $100.0 \%$ & $0.0 \%$ & $0.0 \%$ & $0.0 \%$ & $0.0 \%$ & 1.00003 \\
\hline 20 & $100.0 \%$ & $0.0 \%$ & $0.0 \%$ & $0.0 \%$ & $0.0 \%$ & 1.00006 \\
\hline 21 & $100.0 \%$ & $0.0 \%$ & $0.0 \%$ & $0.0 \%$ & $0.0 \%$ & 1.00006 \\
\hline 22 & $100.0 \%$ & $0.0 \%$ & $0.0 \%$ & $0.0 \%$ & $0.0 \%$ & 1.00006 \\
\hline 23 & $100.0 \%$ & $0.0 \%$ & $0.0 \%$ & $0.0 \%$ & $0.0 \%$ & 1.00009 \\
\hline 24 & $100.0 \%$ & $0.0 \%$ & $0.0 \%$ & $0.0 \%$ & $0.0 \%$ & 1.00009 \\
\hline 25 & $100.0 \%$ & $0.0 \%$ & $0.0 \%$ & $0.0 \%$ & $0.0 \%$ & 1.00009 \\
\hline 26 & $100.0 \%$ & $0.0 \%$ & $0.0 \%$ & $0.0 \%$ & $0.0 \%$ & 1.00010 \\
\hline 27 & $100.0 \%$ & $0.0 \%$ & $0.0 \%$ & $0.0 \%$ & $0.0 \%$ & 1.00010 \\
\hline 28 & $100.0 \%$ & $0.0 \%$ & $0.0 \%$ & $0.0 \%$ & $0.0 \%$ & 1.00010 \\
\hline 29 & $100.0 \%$ & $0.0 \%$ & $0.0 \%$ & $0.0 \%$ & $0.0 \%$ & 1.00011 \\
\hline 30 & $100.0 \%$ & $0.0 \%$ & $0.0 \%$ & $0.0 \%$ & $0.0 \%$ & 1.00011 \\
\hline 31 & $99.7 \%$ & $0.1 \%$ & $0.1 \%$ & $0.1 \%$ & $0.1 \%$ & 1.00600 \\
\hline 32 & $99.4 \%$ & $0.3 \%$ & $0.1 \%$ & $0.1 \%$ & $0.1 \%$ & 1.01234 \\
\hline 33 & $99.4 \%$ & $0.1 \%$ & $0.2 \%$ & $0.1 \%$ & $0.3 \%$ & 1.01235 \\
\hline 34 & $99.4 \%$ & $0.1 \%$ & $0.2 \%$ & $0.3 \%$ & $0.1 \%$ & 1.01236 \\
\hline 35 & $0.0 \%$ & $100.0 \%$ & $0.0 \%$ & $0.0 \%$ & $0.0 \%$ & 1.00000 \\
\hline 36 & $1.2 \%$ & $98.5 \%$ & $0.1 \%$ & $0.1 \%$ & $0.1 \%$ & 1.03081 \\
\hline 37 & $0.0 \%$ & $0.0 \%$ & $100.0 \%$ & $0.0 \%$ & $0.0 \%$ & 1.00000 \\
\hline 38 & $1.2 \%$ & $0.1 \%$ & $98.5 \%$ & $0.1 \%$ & $0.1 \%$ & 1.03079 \\
\hline 39 & $0.0 \%$ & $0.0 \%$ & $0.0 \%$ & $100.0 \%$ & $0.0 \%$ & 1.00000 \\
\hline 40 & $1.2 \%$ & $0.1 \%$ & $0.1 \%$ & $98.5 \%$ & $0.1 \%$ & 1.03078 \\
\hline 41 & $0.0 \%$ & $0.0 \%$ & $0.0 \%$ & $0.0 \%$ & $100.0 \%$ & 1.00000 \\
\hline 42 & $1.2 \%$ & $0.1 \%$ & $0.1 \%$ & $0.1 \%$ & $98.5 \%$ & 1.03079 \\
\hline 43 & $44.5 \%$ & $54.4 \%$ & $0.3 \%$ & $0.3 \%$ & $0.3 \%$ & 2.02123 \\
\hline 44 & $40.7 \%$ & $57.5 \%$ & $0.7 \%$ & $0.3 \%$ & $0.9 \%$ & 2.01393 \\
\hline 45 & $40.7 \%$ & $57.5 \%$ & $0.5 \%$ & $0.9 \%$ & $0.3 \%$ & 2.01391 \\
\hline 46 & $44.5 \%$ & $0.3 \%$ & $54.4 \%$ & $0.3 \%$ & $0.3 \%$ & 2.02115 \\
\hline 47 & $40.7 \%$ & $1.0 \%$ & $57.5 \%$ & $0.4 \%$ & $0.4 \%$ & 2.01387 \\
\hline 48 & $40.7 \%$ & $0.2 \%$ & $57.5 \%$ & $0.8 \%$ & $0.8 \%$ & 2.01387 \\
\hline 49 & $44.5 \%$ & $0.3 \%$ & $0.3 \%$ & $54.5 \%$ & $0.3 \%$ & 2.02111 \\
\hline 50 & $40.7 \%$ & $0.5 \%$ & $1.0 \%$ & $57.5 \%$ & $0.4 \%$ & 2.01388 \\
\hline 51 & $40.7 \%$ & $0.7 \%$ & $0.2 \%$ & $57.5 \%$ & $0.8 \%$ & 2.01389 \\
\hline 52 & $44.5 \%$ & $0.3 \%$ & $0.3 \%$ & $0.3 \%$ & $54.4 \%$ & 2.02115 \\
\hline 53 & $40.7 \%$ & $0.9 \%$ & $0.3 \%$ & $0.6 \%$ & $57.5 \%$ & 2.01389 \\
\hline 54 & $40.7 \%$ & $0.3 \%$ & $0.9 \%$ & $0.6 \%$ & $57.5 \%$ & 2.01387 \\
\hline
\end{tabular}




\section{S2 Generalized Fragment Natural Orbitals (FNO): Eigenvectors}

Notice that the numbering of FNOs does not coincide with that in the text.

\section{$\mathrm{S} 2.1 \quad \mathrm{CH}_{4}$}

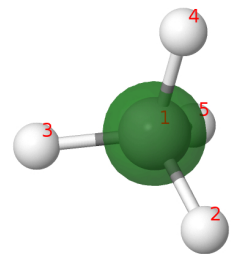

FNO 1

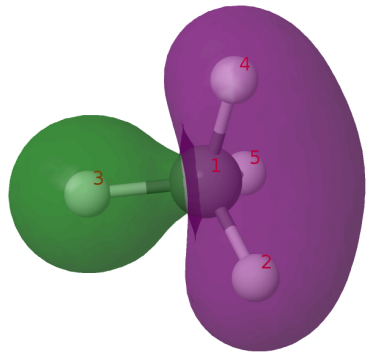

FNO 2

Figure S1: $|\varphi|=0.05$ a.u. isosurface FNOs of the $\mathrm{CH}_{4}$ molecule at the restricted Hartree-Fock RHF//def2-QZVPPD level of calculation.

$\mathrm{S2.2} \quad \mathrm{SO}_{4}^{2-}$

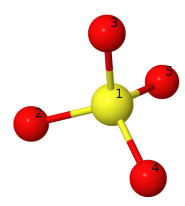

FNO 1

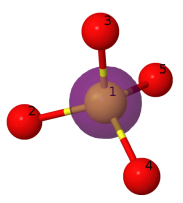

FNO 2

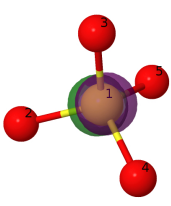

FNO 3

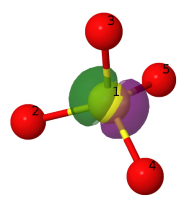

FNO 4

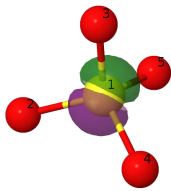

FNO 5

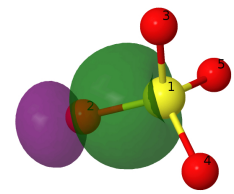

FNO 14

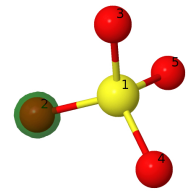

FNO 6

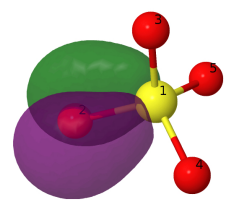

FNO 16

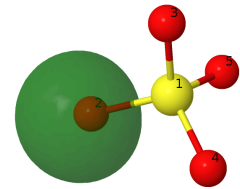

FNO 7

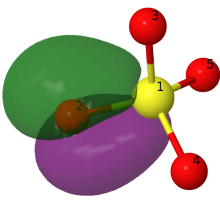

FNO 17

Figure S2: $|\varphi|=0.05$ a.u. isosurface of the FNOs of the $\mathrm{SO}_{4}^{2-}$ molecule at the B3LYP//def2-QZVPPD level of calculation. 


\section{S2.3 $\mathbf{N}_{2} \mathbf{H}_{2}$}

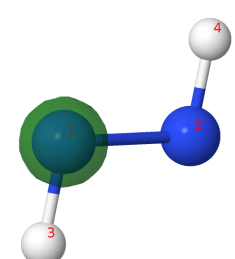

FNO 1

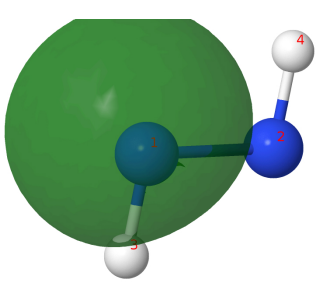

FNO 2

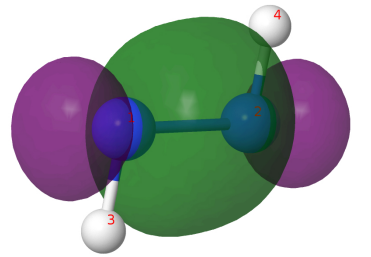

FNO 5

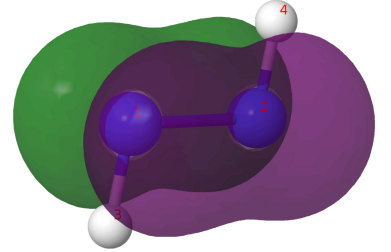

FNO 6

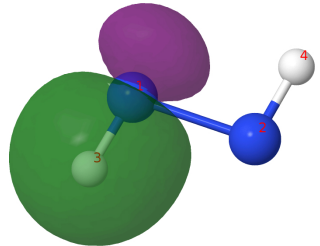

FNO 7

Figure S3: $|\varphi|=0.05$ a.u. isosurface of the FNOs of the $\mathrm{N}_{2} \mathrm{H}_{2}$ molecule at the CAS[12,8]//6-311G(d) level of calculation. 
S2.4 The cis-butadiene plus ethylene Diels-Alder (DA) reaction

\section{S2.4.1 Reactants}

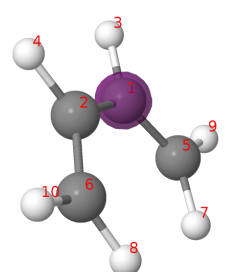

FNO 1

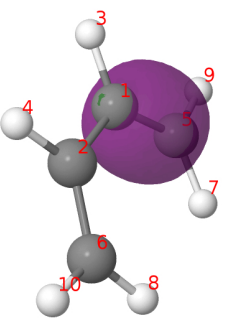

FNO 9
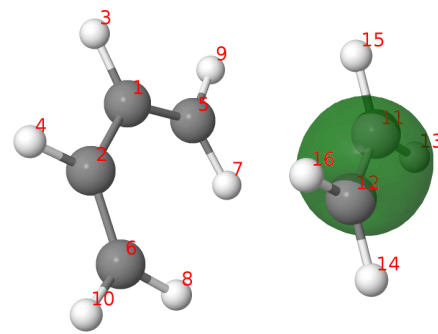

FNO 16

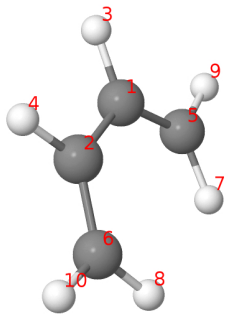

FNO 19
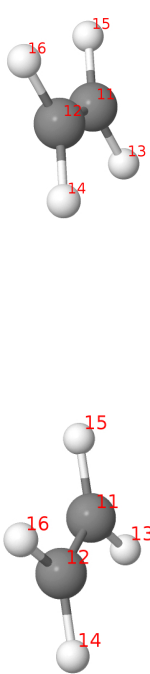

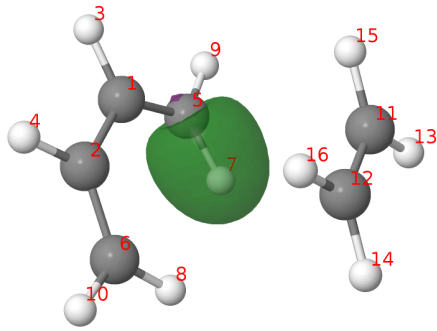

FNO 12
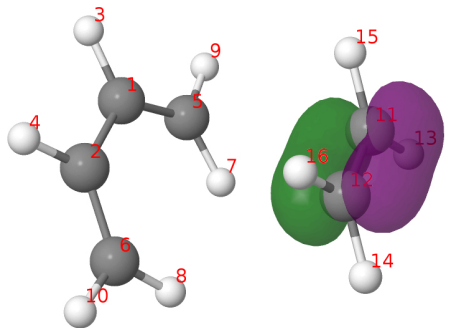

FNO 17
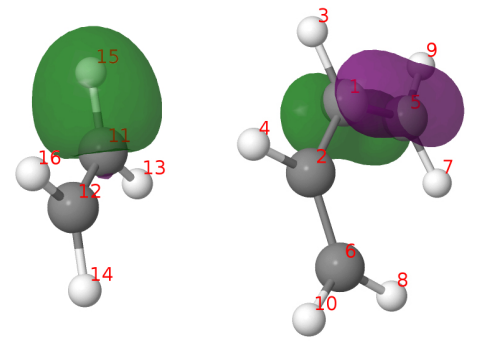

FNO 22

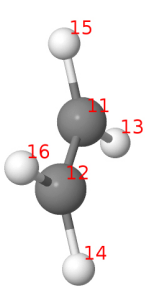

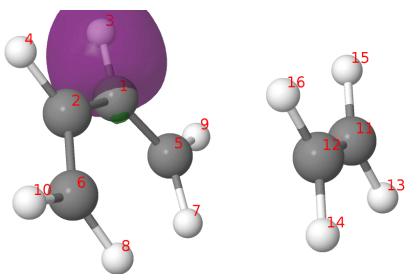

FNO 8

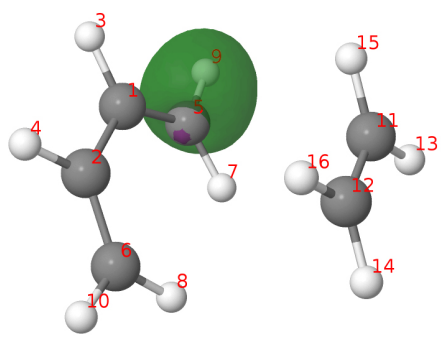

FNO 13

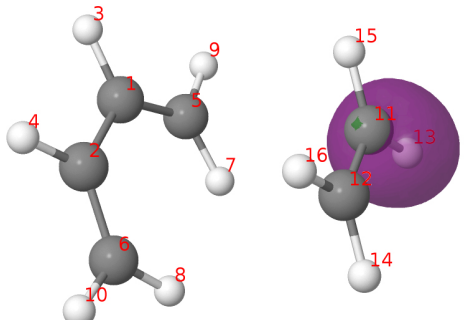

FNO 18
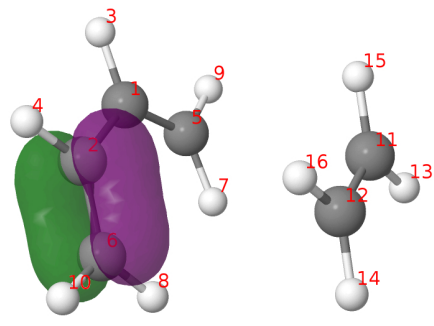

FNO 23

Figure S4: $|\varphi|=0.1$ a.u. isosurface of the FNOs of the reactants at the B3LYP//aug-cc-pVDZ level of calculation. 
S2.4.2 Transition state

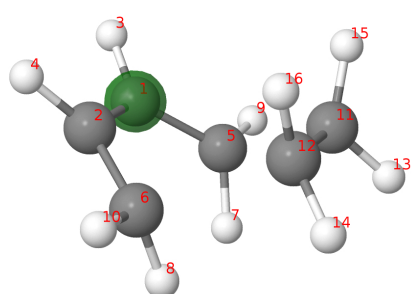

FNO 1

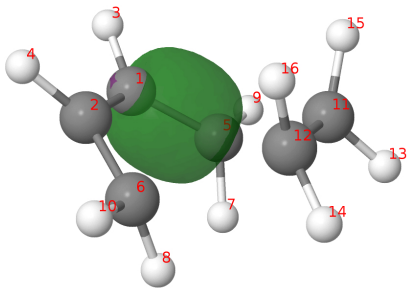

FNO 9

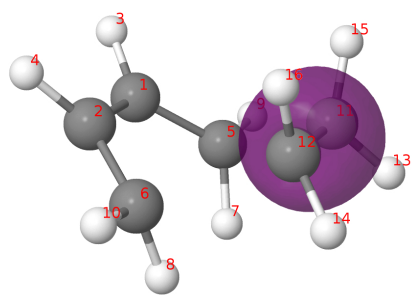

FNO 16

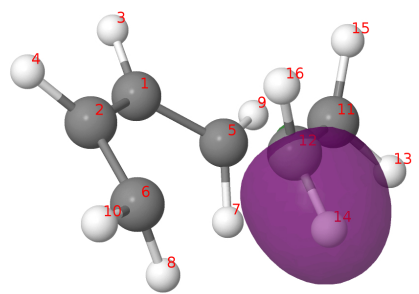

FNO 19

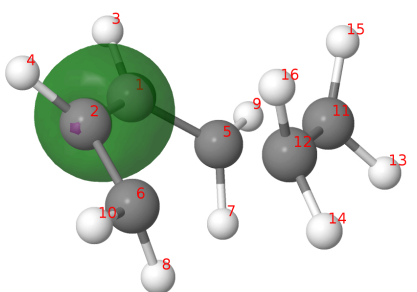

FNO 7

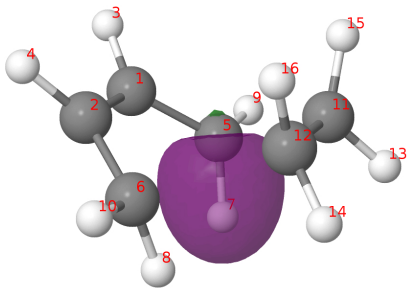

FNO 12

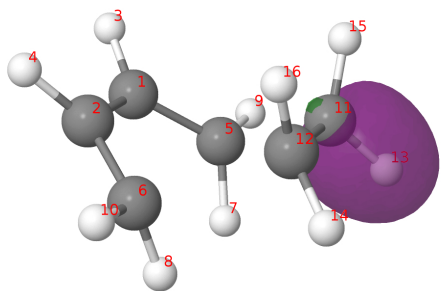

FNO 17

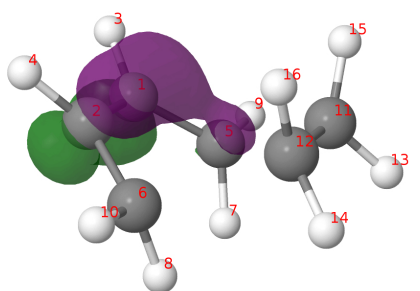

FNO 20

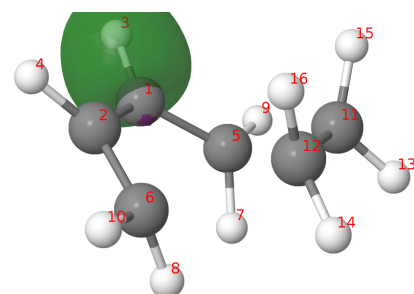

FNO 8

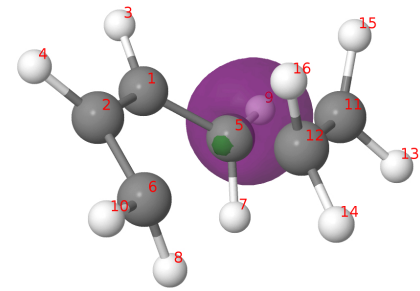

FNO 13

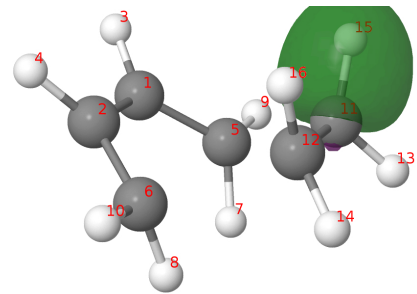

FNO 18

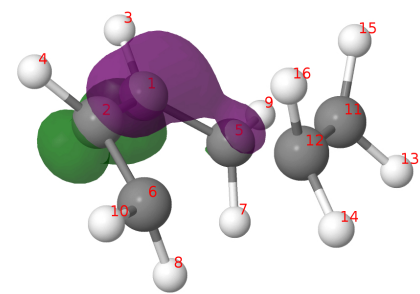

FNO 21

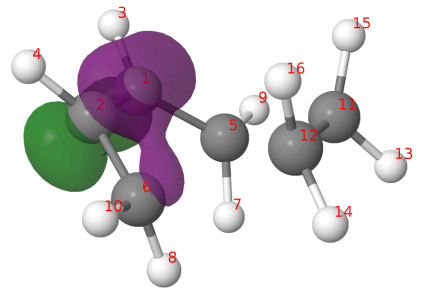

FNO 22

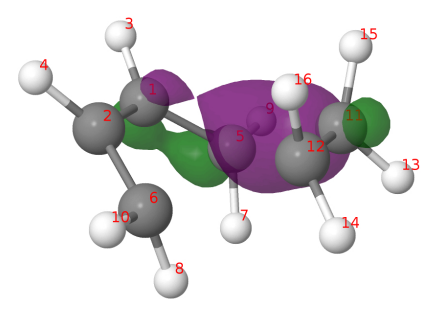

FNO 23

Figure S5: $|\varphi|=0.1$ a.u. isosurface of the FNOs of the transition state at the B3LYP//aug-cc-pVDZ level of calculation. 


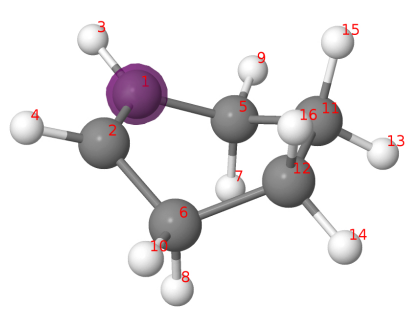

FNO 1

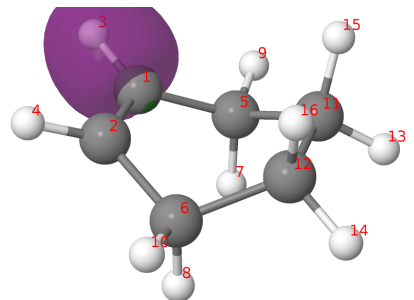

FNO 9

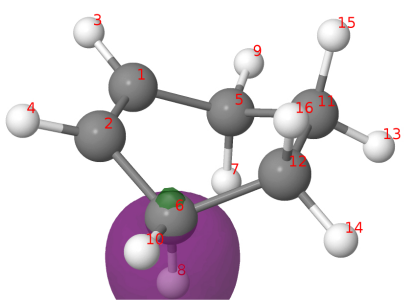

FNO 16

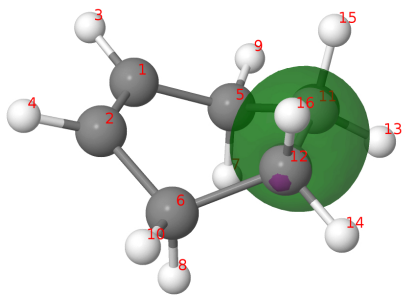

FNO 19

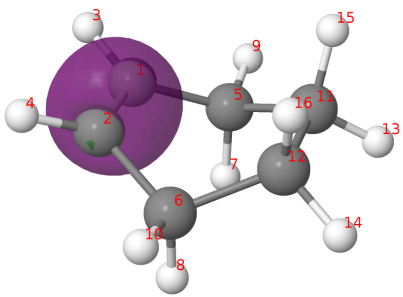

FNO 7

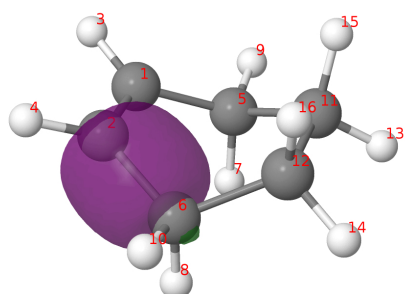

FNO 12

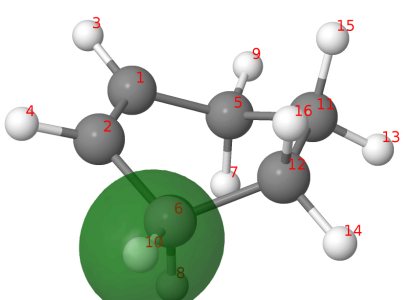

FNO 17

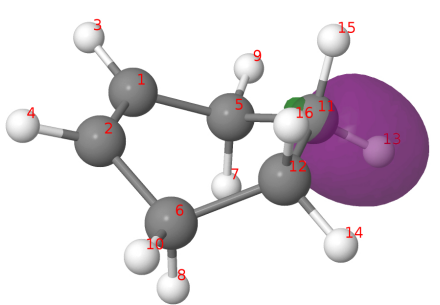

FNO 20

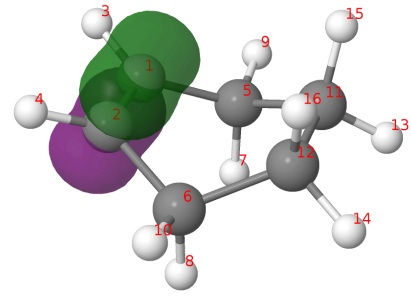

FNO 8

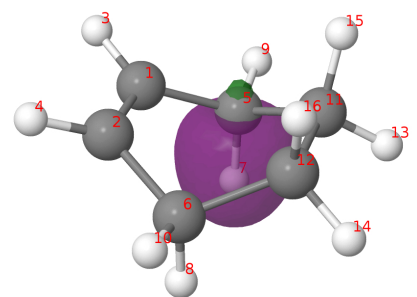

FNO 13

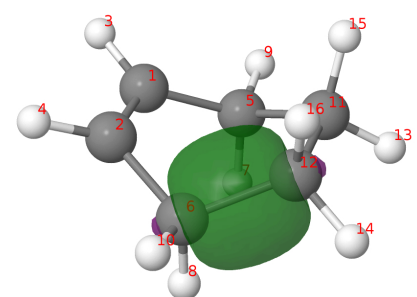

FNO 18

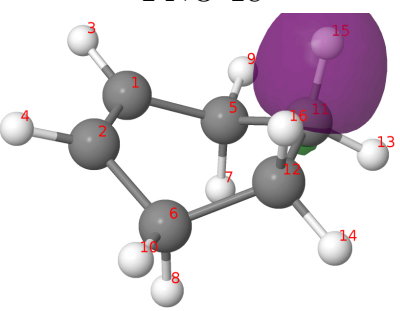

FNO 21

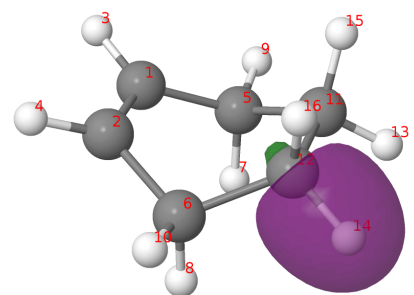

FNO 22

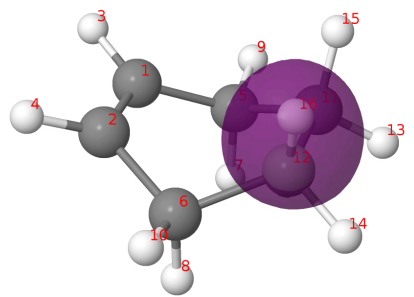

FNO 23

Figure S6: $|\varphi|=0.1$ a.u. isosurface of the FNOs of the products at the B3LYP//aug-cc-pVDZ level of calculation. 
S2.5 The $\mathbf{F}^{-}+\mathrm{CH}_{3} \mathbf{F} \rightarrow \mathrm{FCH}_{3}+\mathbf{F}^{-}$reaction

S2.5.1 Reactants/Products

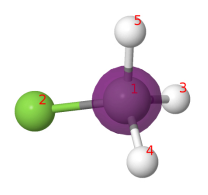

FNO 1

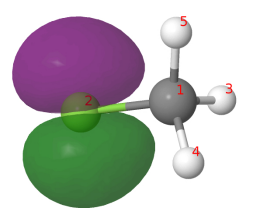

FNO 4

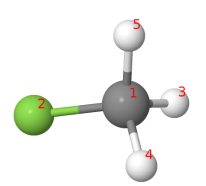

FNO 7

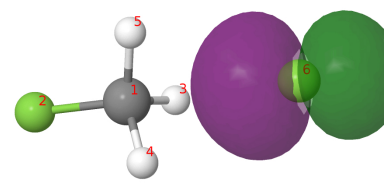

FNO 10

0
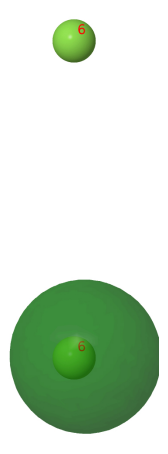

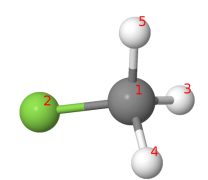

FNO 8

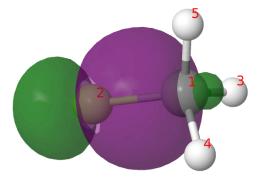

FNO 11

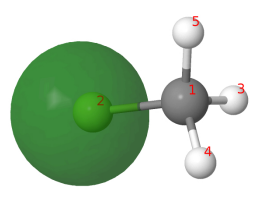

FNO 3

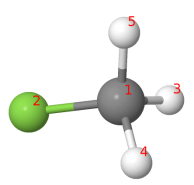

FNO 6

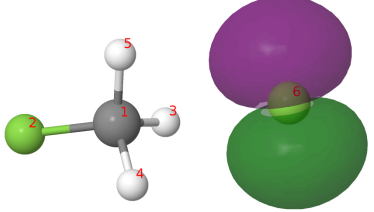

FNO 9

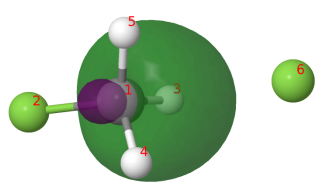

FNO 12

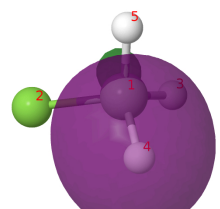

FNO 13

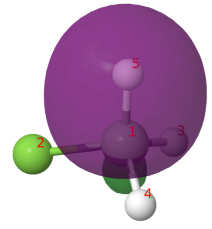

FNO 14

Figure S7: $|\varphi|=0.05$ a.u. isosurface of the FNOs of the reactants at the B3LYP//aug-cc-pVDZ level of calculation. 


\section{S2.5.2 Transition State}

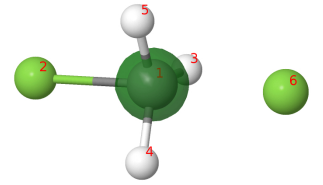

FNO 1

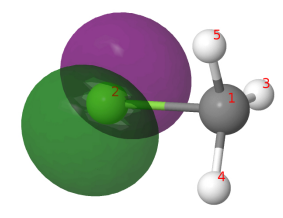

FNO 4

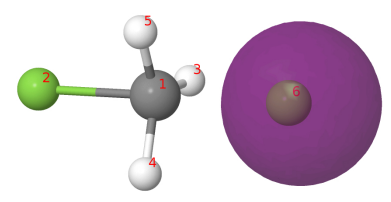

FNO 7

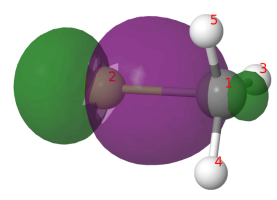

FNO 10

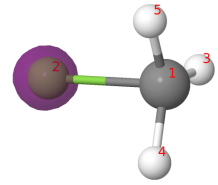

FNO 2

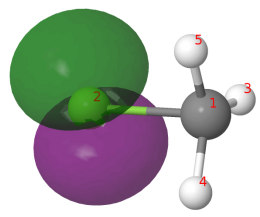

FNO 5

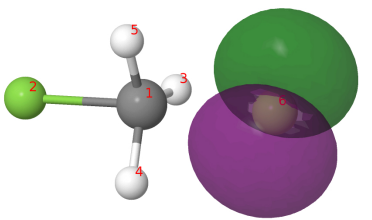

FNO 8

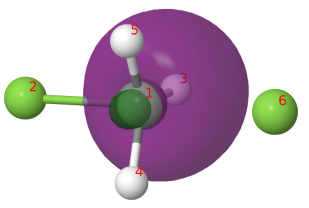

FNO 11

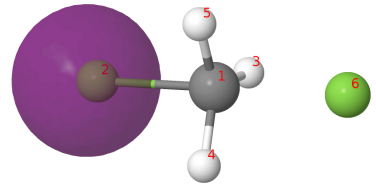

FNO 3

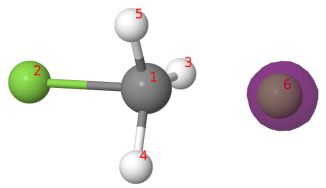

FNO 6

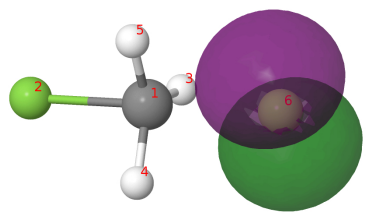

FNO 9

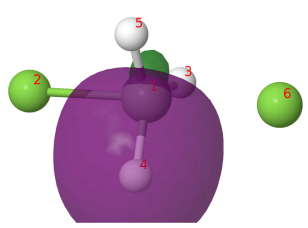

FNO 12

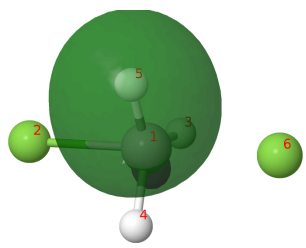

FNO 13

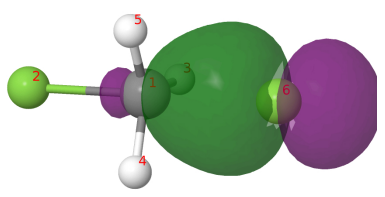

FNO 14

Figure S8: $|\varphi|=0.05$ a.u. isosurface of the FNOs of the reactants at the B3LYP//aug-cc-pVDZ level of calculation. 
S2.6 FeF $_{6}^{3-}$ complex in $O h$ (high spin) and $D_{4 h}$ (low spin) multielectron states.

S2.6.1 $\quad \mathbf{F e F}_{6}^{3-}$ Oh High spin, $\left(\mathbf{t}_{2 g}^{3} \mathbf{e}_{g}^{2}-{ }^{6} A_{1 g}\right), \alpha-$ orbitals

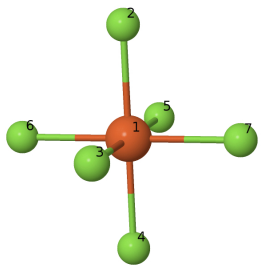

FNO 1

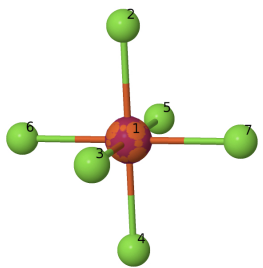

FNO 5

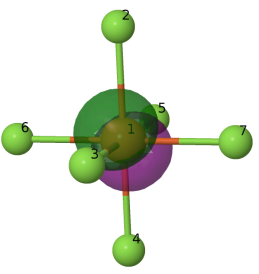

FNO 9

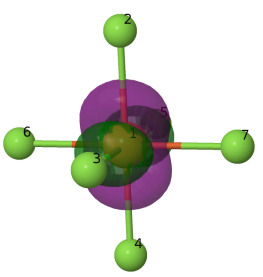

FNO 13

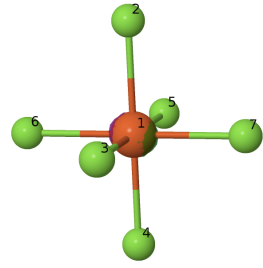

FNO 2

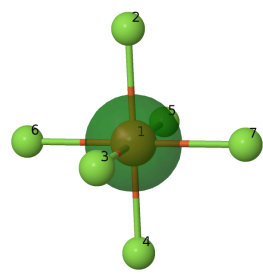

FNO 6

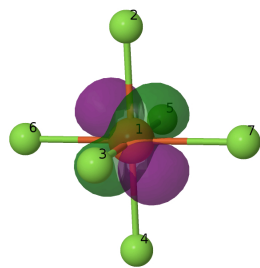

FNO 10

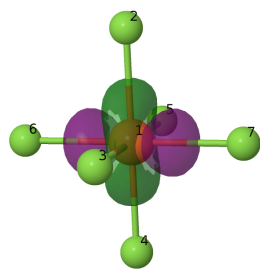

FNO 14

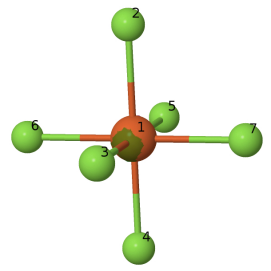

FNO 3

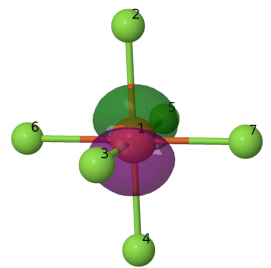

FNO 7

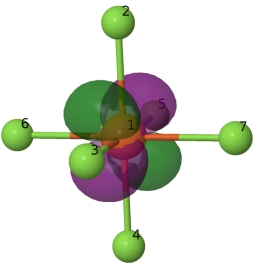

FNO 11

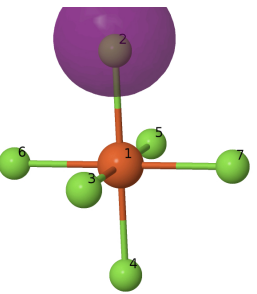

FNO 15

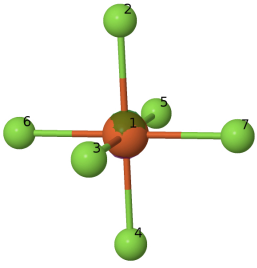

FNO 4

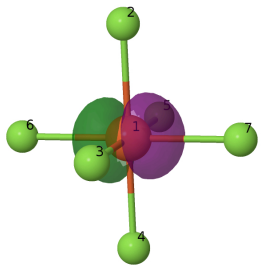

FNO 8

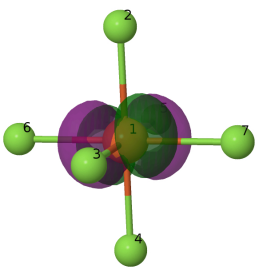

FNO 12

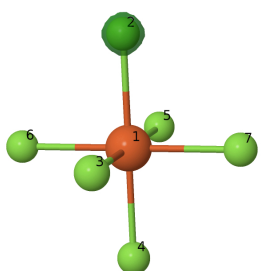

FNO 16

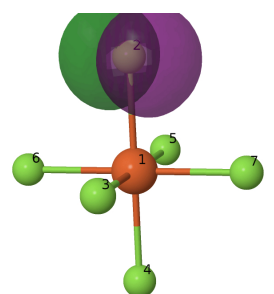

FNO 17

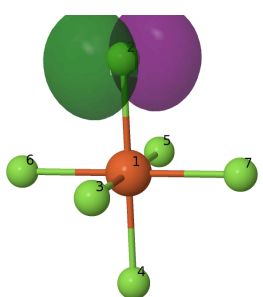

FNO 18

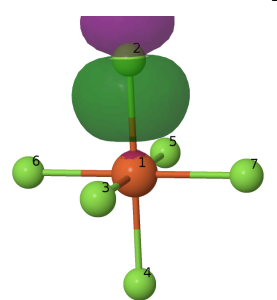

FNO 19

Figure S9: $|\varphi|=0.05$ a.u. isosurface of the FNOs of the $\mathrm{FeF}_{6}^{3-}$ complex at the unrestricted DFT M06-2X//aug-ccpVDZ level in the $O h$ high spin $\left(\mathrm{t}_{2 g}^{3} \mathrm{e}_{g}^{2}-{ }^{6} A_{1 g}\right), \alpha$-orbitals. 
S2.6.2 $\mathbf{F e F}_{6}^{3-}$ Oh High spin, $\left(\mathbf{t}_{2 g}^{3} \mathbf{e}_{g}^{2}-{ }^{6} A_{1 g}\right), \beta-$ orbitals

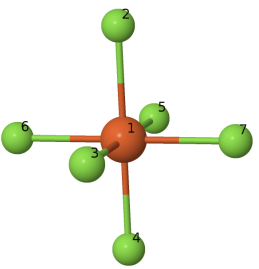

FNO 45

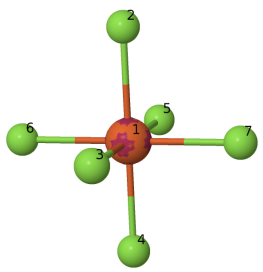

FNO 49

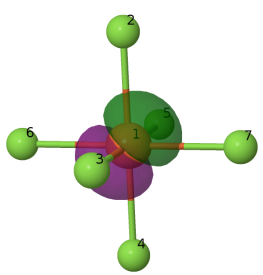

FNO 53

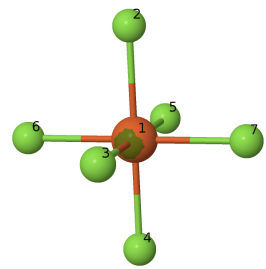

FNO 46

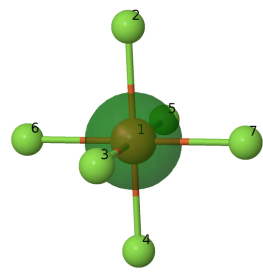

FNO 50

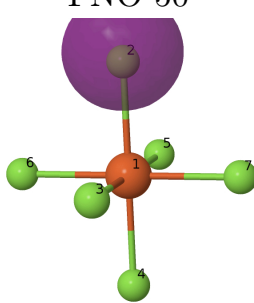

FNO 54

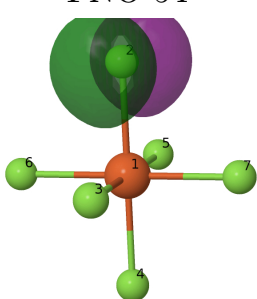

FNO 57

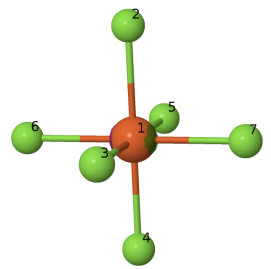

FNO 47

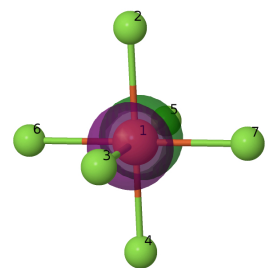

FNO 51

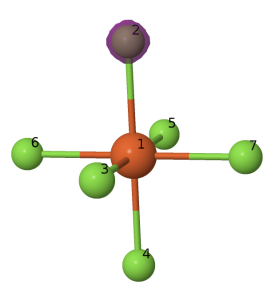

FNO 55

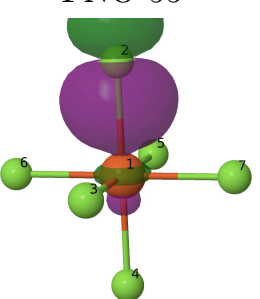

FNO 58

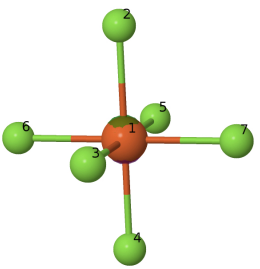

FNO 48

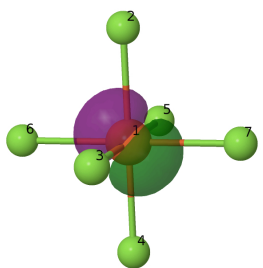

FNO 52

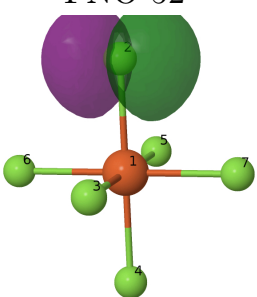

FNO 56

Figure S10: $|\varphi|=0.05$ a.u. isosurface of the FNOs of the $\mathrm{FeF}_{6}^{3-}$ complex at the unrestricted DFT M06-2X//aug-cc$\mathrm{pVDZ}$ level in the $O h$ high spin $\left(\mathrm{t}_{2 g}^{3} \mathrm{e}_{g}^{2}-{ }^{6} A_{1 g}\right), \beta$-orbitals. 
S2.6.3 $\mathbf{F e F}_{6}^{3-} D_{4 h}$ Low spin $\left(\mathbf{e}_{g}^{4} \mathbf{b}_{2 g}^{1}-{ }^{2} B_{2 g}\right), \alpha-$ orbitals

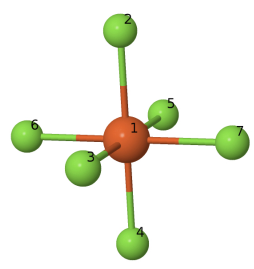

FNO 1

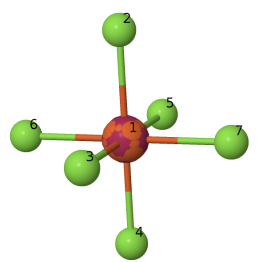

FNO 5

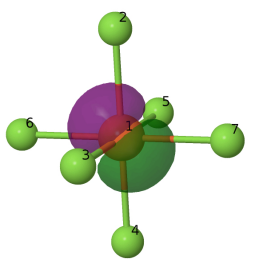

FNO 9

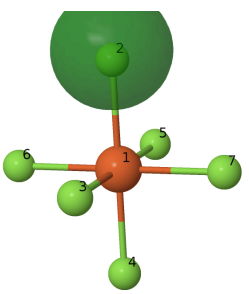

FNO 13

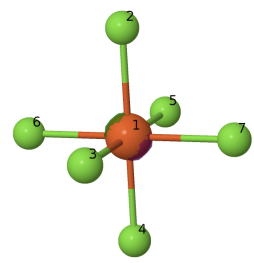

FNO 2

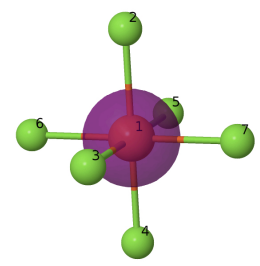

FNO 6
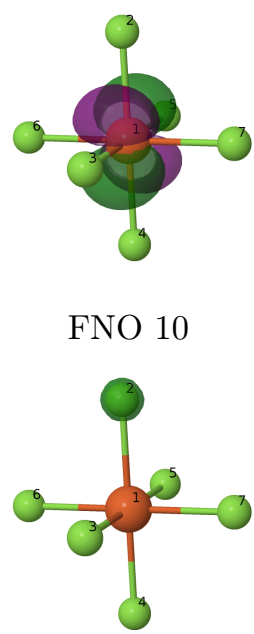

FNO 14

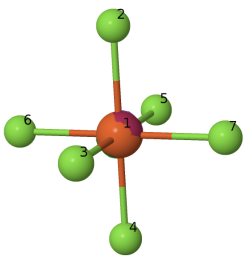

FNO 3

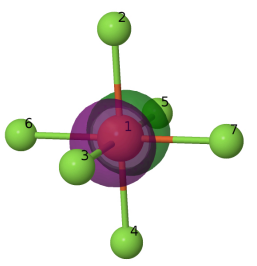

FNO 7

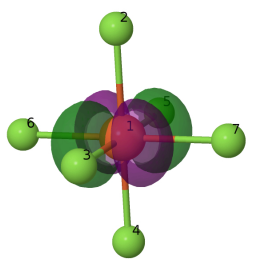

FNO 11

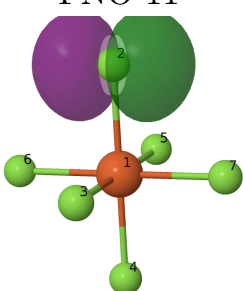

FNO 15

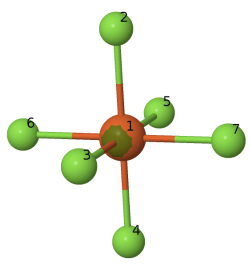

FNO 4

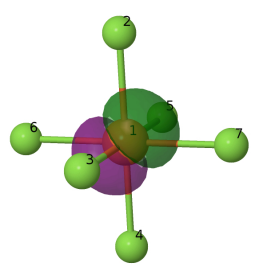

FNO 8
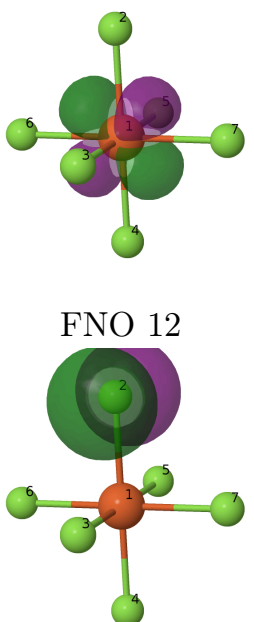

FNO 16

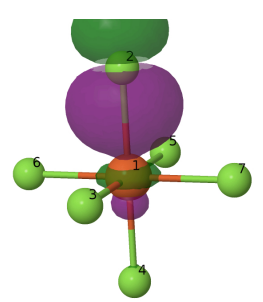

FNO 17

Figure S11: $|\varphi|=0.05$ a.u. isosurface of the FNOs of the $\mathrm{FeF}_{6}^{3-}$ complex at the unrestricted DFT M06-2X//aug-ccpVDZ level in the $D_{4 h}$ low spin $\left(\mathrm{e}_{g}^{4} \mathrm{~b}_{2 g}^{1}-{ }^{2} B_{2 g}\right), \alpha$-orbitals. 
S2.6.4 $\quad \mathbf{F e F}_{6}^{3-} D_{4 h}$ Low spin $\left(\mathbf{e}_{g}^{4} \mathbf{b}_{2 g}^{1}-{ }^{2} B_{2 g}\right) \beta-$ orbitals

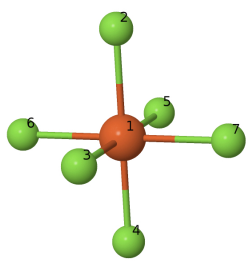

FNO 43

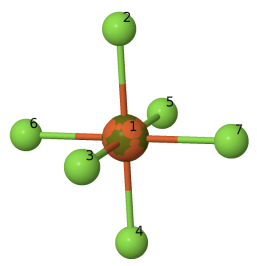

FNO 47

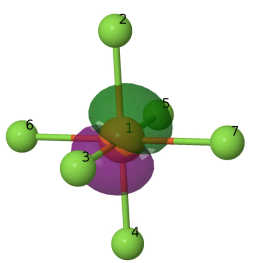

FNO 51

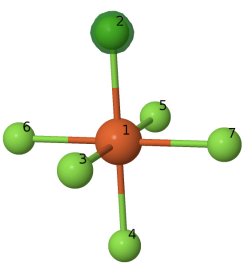

FNO 55

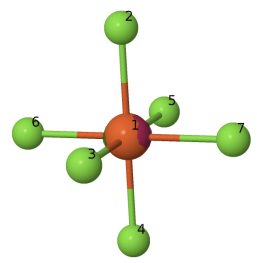

FNO 44

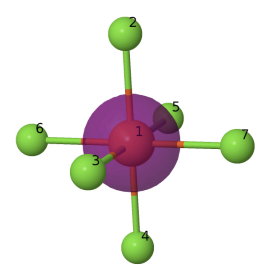

FNO 48

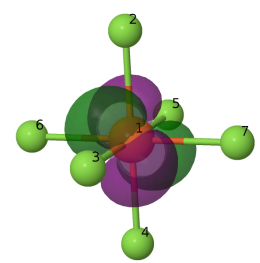

FNO 52

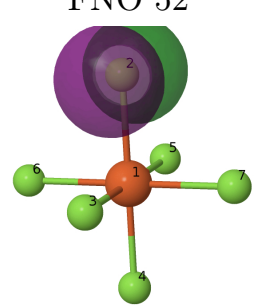

FNO 56

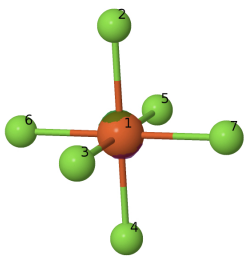

FNO 45

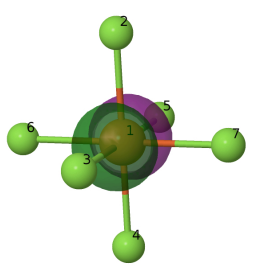

FNO 49

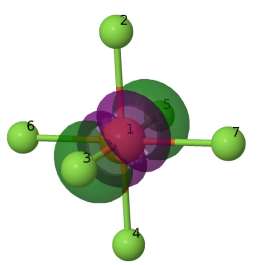

FNO 53

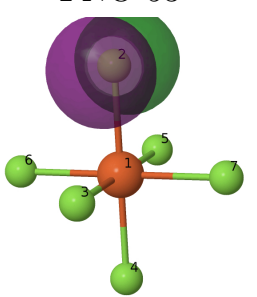

FNO 57

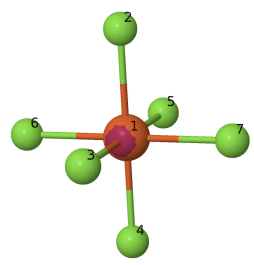

FNO 46

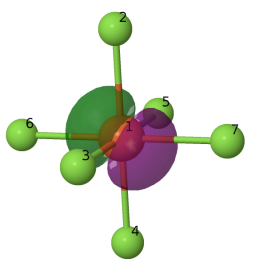

FNO 50

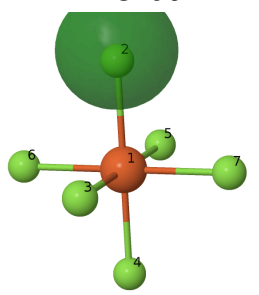

FNO 54

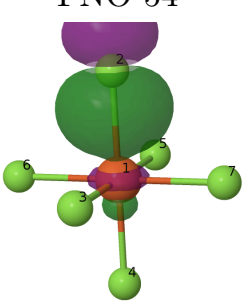

FNO 58

Figure S12: $|\varphi|=0.05$ a.u. isosurface of the FNOs of the $\mathrm{FeF}_{6}^{3-}$ complex at the unrestricted DFT M06-2X//aug-ccpVDZ level in the $D_{4 h}$ low spin $\left(\mathrm{e}_{g}^{4} \mathrm{~b}_{2 g}^{1}-{ }^{2} B_{2 g}\right), \beta$-orbitals. 
S2.7 $\mathrm{PtO}_{4}^{2+}$

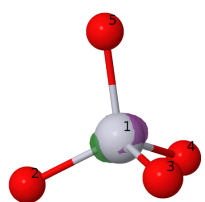

FNO 16

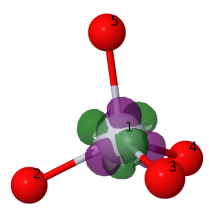

FNO 20

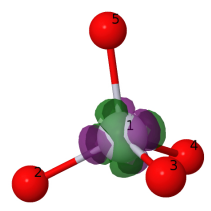

FNO 24

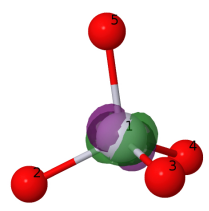

FNO 28

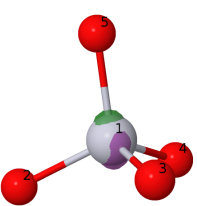

FNO 17

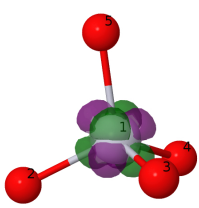

FNO 21

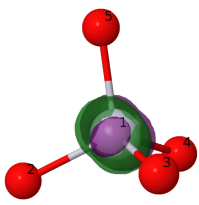

FNO 25

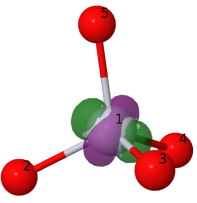

FNO 29

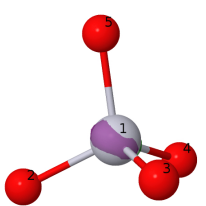

FNO 18

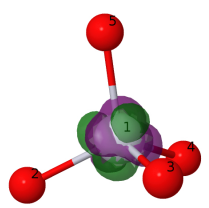

FNO 22

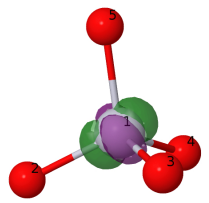

FNO 26

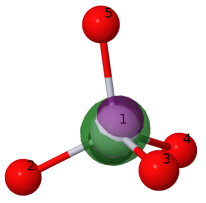

FNO 30

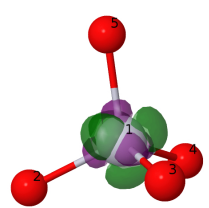

FNO 19

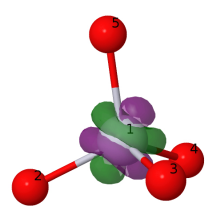

FNO 23

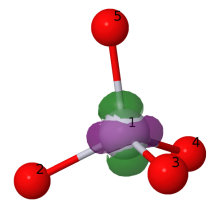

FNO 27

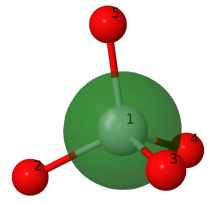

FNO 31

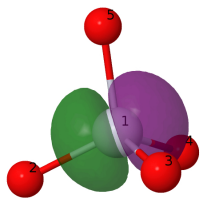

FNO 32

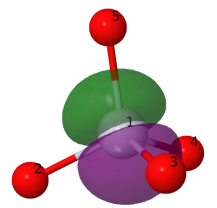

FNO 33

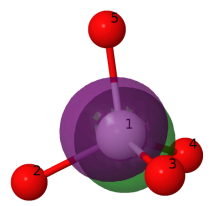

FNO 34

Figure S13: $|\varphi|=0.05$ a.u. isosurface of the FNOs of the $\mathrm{PtO}_{4}^{2+}$ complex as obtained from heat bath CI (HCI) calculations using ADZP $(\mathrm{Pt})$ def2-QZVPPD $(\mathrm{O})$ basis sets. These FNOs correspond to the external shell of the Pt atom. 


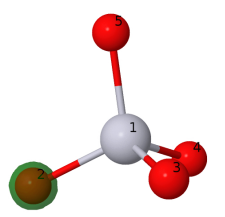

FNO 35

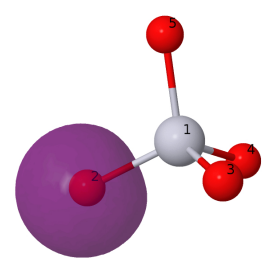

FNO 36

Figure S14: $|\varphi|=0.05$ a.u. isosurface of the FNOs of the $\mathrm{PtO}_{4}^{2+}$ complex as obtained from heat bath CI (HCI) calculations using ADZP (Pt) def2-QZVPPD $(\mathrm{O})$ basis sets. These FNOs are mainly localized on the oxygen atom.

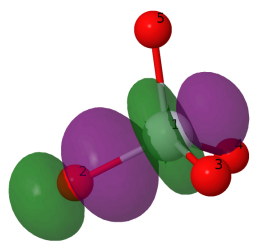

FNO 43

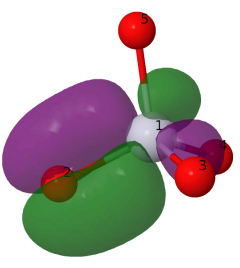

FNO 44

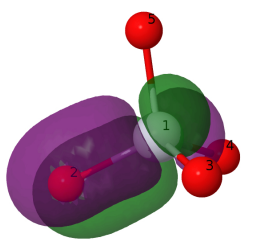

FNO 45

Figure S15: $|\varphi|=0.05$ a.u. isosurface of the FNOs of the $\mathrm{PtO}_{4}^{2+}$ complex as obtained from heat batch CI (HCI) calculations using ADZP (Pt) def2-QZVPPD (O) basis sets. These FNOs correspond to the $\sigma$ (left) and $\pi$ (center and right) Pt-O bonds.

\section{S3 Cartesian coordinates}

\section{S3.1 $\mathrm{CH}_{4}$}

$\begin{array}{rrrr}\text { C1 } & 0.00000000 & 0.00000000 & 0.00000000 \\ \text { H2 } & 1.20000000 & 1.20000000 & 1.20000000 \\ \text { H3 } & -1.20000000 & -1.20000000 & 1.20000000 \\ \text { H4 } & -1.20000000 & 1.20000000 & -1.20000000 \\ \text { H5 } & 1.20000000 & -1.20000000 & -1.20000000\end{array}$

\section{S3.2 $\quad \mathrm{SO}_{4}^{2-}$}

$\begin{array}{rrrr}\text { S1 } & 0.00000000 & 0.00000000 & 0.00000000 \\ 02 & -1.63990201 & -1.63990201 & 1.63990201 \\ 03 & 1.63990201 & 1.63990201 & 1.63990201 \\ 04 & 1.63990201 & -1.63990201 & -1.63990201 \\ 05 & -1.63990201 & 1.63990201 & -1.63990201\end{array}$

\section{S3.3 $\quad \mathbf{N}_{2} \mathbf{H}_{2}$}
$\begin{array}{llll}\text { N1 } & -1.51178079 & 0.00000000 & 0.00000000\end{array}$
$\begin{array}{llll}\text { N2 } & 1.51178079 & 0.00000000 & 0.00000000\end{array}$
H3 $-1.78851470-1.89098960 \quad 0.00000000$
H4 $1.78851470 \quad 1.89098960 \quad 0.00000000$

\section{S3.4 The cis-butadiene plus ethylene Diels-Alder (DA) reaction}

\section{S3.4.1 Reactants}

$\begin{array}{lrrr}\text { C1 } & -2.74331542 & 0.56332736 & -1.38838178 \\ \text { C2 } & -2.74350439 & 0.56313839 & 1.38857076 \\ \text { H3 } & -3.76225574 & 2.13274490 & -2.26559265 \\ \text { H4 } & -3.76282266 & 2.13198901 & 2.26578162 \\ \text { C5 } & -1.62459755 & -1.12665472 & -2.91981584 \\ \text { C6 } & -1.62440858 & -1.12703266 & 2.91962686\end{array}$




$\begin{array}{lrrr}\text { H7 } & -0.57731133 & -2.74728384 & -2.19964121 \\ \text { H8 } & -0.57674441 & -2.74709487 & 2.19945224 \\ \text { H9 } & -1.73854803 & -0.93484751 & -4.96676717 \\ \text { H10 } & -1.73835906 & -0.93560340 & 4.96676717 \\ \text { C11 } & 4.17667268 & 0.59734243 & -1.26158116 \\ \text { C12 } & 4.17686165 & 0.59809832 & 1.26177013 \\ \text { H13 } & 4.86906833 & -1.01440498 & -2.34571704 \\ \text { H14 } & 4.86944628 & -1.01289320 & 2.34666190 \\ \text { H15 } & 3.48106449 & 2.20549936 & -2.34458320 \\ \text { H16 } & 3.48125347 & 2.20682217 & 2.34382731\end{array}$

\section{S3.4.2 Transition state}

$\begin{array}{lrrr}\text { C1 } & -2.42508554 & 0.62115298 & -1.29843082 \\ \text { C2 } & -2.42508554 & 0.62115298 & 1.29843082 \\ \text { H3 } & -3.50090662 & 2.06320298 & -2.30962327 \\ \text { H4 } & -3.50090662 & 2.06320298 & 2.30962327 \\ \text { C5 } & -0.62644421 & -0.87021888 & -2.66167925 \\ \text { C6 } & -0.62625524 & -0.87021888 & 2.66149027 \\ \text { H7 } & -0.33882789 & -2.81984932 & -2.05262052 \\ \text { H8 } & -0.33863892 & -2.81984932 & 2.05243154 \\ \text { H9 } & -0.58978352 & -0.66593949 & -4.71637846 \\ \text { H10 } & -0.58959455 & -0.66612846 & 4.71618949 \\ \text { C11 } & 2.84214809 & 0.29101782 & -1.37496473 \\ \text { C12 } & 2.84195912 & 0.29120680 & 1.37496473 \\ \text { H13 } & 3.98467651 & -1.15443369 & -2.30489895 \\ \text { H14 } & 3.98467651 & -1.15405574 & 2.30508793 \\ \text { H15 } & 2.93285495 & 2.12480805 & -2.31151300 \\ \text { H16 } & 2.93266597 & 2.12499703 & 2.31132402\end{array}$

\section{S3.4.3 Products}

$\begin{array}{lrrr}\text { C1 } & -2.34571704 & 0.63362517 & -1.26687239 \\ \text { C2 } & -2.34590601 & 0.63362517 & 1.26687239 \\ \text { H3 } & -3.83425431 & 1.56280351 & -2.35176416 \\ \text { H4 } & -3.83387636 & 1.56280351 & 2.35157519 \\ \text { C5 } & -0.20541323 & -0.68615956 & -2.60801102 \\ \text { C6 } & -0.20560220 & -0.68615956 & 2.60801102 \\ \text { H7 } & -0.42632221 & -2.75049637 & -2.39314916 \\ \text { H8 } & -0.42632221 & -2.75030740 & 2.39314916 \\ \text { H9 } & -0.23186940 & -0.30519077 & -4.64740346 \\ \text { H10 } & -0.23130248 & -0.30481282 & 4.64740346 \\ \text { C11 } & 2.36782683 & 0.10903720 & -1.46812823 \\ \text { C12 } & 2.36801581 & 0.10884822 & 1.46812823 \\ \text { H13 } & 3.85277362 & -1.14706376 & -2.19831840 \\ \text { H14 } & 3.85315157 & -1.14725273 & 2.19812943 \\ \text { H15 } & 2.81720371 & 2.01520394 & -2.15806723 \\ \text { H16 } & 2.81758165 & 2.01501497 & 2.15825621\end{array}$

S3.5 The $\mathbf{F}^{-}+\mathrm{CH}_{3} \mathbf{F} \rightarrow \mathbf{F C H}_{3}+\mathbf{F}^{-}$reaction

S3.5.1 Reactants/Products
$\begin{array}{lllll}\text { C1 } & -0.75552138 & 0.00012666 & -0.00021348\end{array}$
$\begin{array}{lllll}\text { F2 } & -3.46379758 & -0.00011228 & 0.00017787\end{array}$
H3 $-0.09624816 \quad 1.83210960-0.67184363$
H4 $-0.09571606-1.49729274-1.25095715$
H5 $-0.09505693-0.33414950 \quad 1.92176713$
F6 $\quad 3.99936974-0.00004631 \quad 0.00007930$

\section{S3.5.2 Transition state}
$\begin{array}{llll}\text { C1 } & 0.00005744 & 0.00037643 & 0.00000000\end{array}$
$\begin{array}{llll}\text { F2 } & 0.00005744 & -0.00013937 & 3.40230072\end{array}$
H3 $-2.03155810 \quad 0.21207733 \quad 0.00000000$ 
H4 $0.83093233-1.86534426 \quad 0.00000000$

H5 $\quad 1.19924724 \quad 1.65351694 \quad 0.00000000$

F6 $\quad 0.00005744-0.00013937-3.40230072$

S3.6 FeF $_{6}^{3-}$ complex in $O h$ (high spin) and $D_{4 h}$ (low spin) multielectron states.

S3.6.1 $\mathbf{F e F}_{6}^{3-} O h$

$\begin{array}{lrrr}\text { Fe1 } & 0.00000000 & 0.00000000 & 0.00000000 \\ \text { F2 } & 0.00000000 & 0.00000000 & 3.77994079 \\ \text { F3 } & 0.00000000 & 3.77994079 & 0.00000000 \\ \text { F4 } & 0.00000000 & 0.00000000 & -3.77994079 \\ \text { F5 } & 0.00000000 & -3.77994079 & 0.00000000 \\ \text { F6 } & 3.77994079 & 0.00000000 & 0.00000000 \\ \text { F7 } & -3.77994079 & 0.00000000 & 0.00000000\end{array}$

S3.6.2 $\quad \mathrm{FeF}_{6}^{3-} D_{4 h}$

$\begin{array}{lrrr}\text { Fe1 } & 0.00000000 & 0.00000000 & 0.00000000 \\ \text { F2 } & 0.00000000 & 3.60197779 & 0.00000000 \\ \text { F3 } & 0.00000000 & 0.00000000 & -3.72221158 \\ \text { F4 } & 0.00000000 & -3.60197779 & 0.00000000 \\ \text { F5 } & 0.00000000 & 0.00000000 & 3.72221158 \\ \text { F6 } & 3.60197779 & 0.00000000 & 0.00000000 \\ \text { F7 } & -3.60197779 & 0.00000000 & 0.00000000\end{array}$

S3.7 $\mathrm{PtO}_{4}^{2+}$

$\begin{array}{lrrr}\text { Pt1 } & 0.00000000 & 0.00000000 & 0.00000000 \\ 02 & -1.86844345 & -1.86844345 & 1.86844345 \\ 03 & 1.86844345 & 1.86844345 & 1.86844345 \\ 04 & 1.86844345 & -1.86844345 & -1.86844345 \\ 05 & -1.86844345 & 1.86844345 & -1.86844345\end{array}$

